\title{
Tailored Silica-Polymer Composites and ABA Type Copolymers: \\ Polymerization Kinetics, Structural Design, and Mechanical Properties
}

\author{
Dissertation \\ zur Erlangung des mathematisch-naturwissenschaftlichen Doktorgrades \\ „Doctor rerum naturalium“ \\ der Georg-August-Universität Göttingen
}

vorgelegt von

Robert Rotzoll

aus Hannover

Göttingen 2011 
Referent: Prof. Dr. Philipp Vana

Korreferent: Prof. Dr. Konrad Samwer

Tag der mündlichen Prüfung: 18. Juli 2011 


\section{Meiner Familie}





\section{Table of Contents}

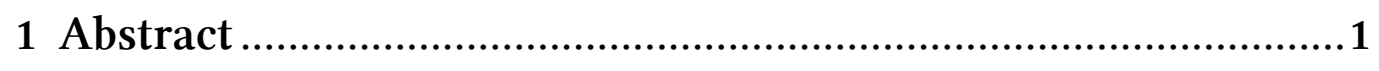

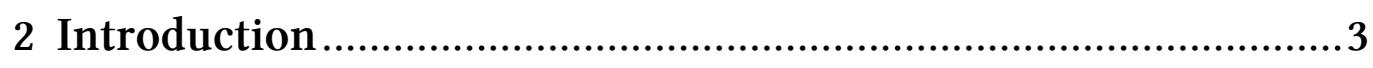

3 Theoretical Background ........................................................

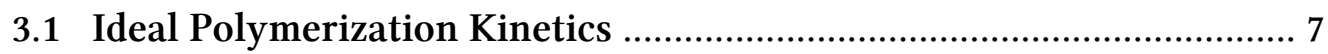

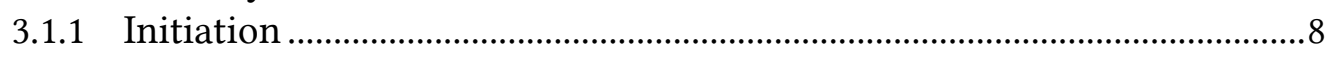

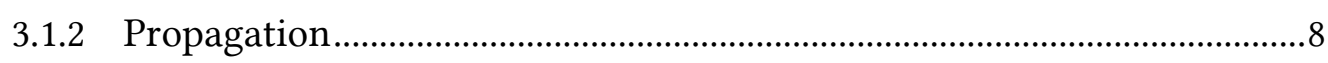

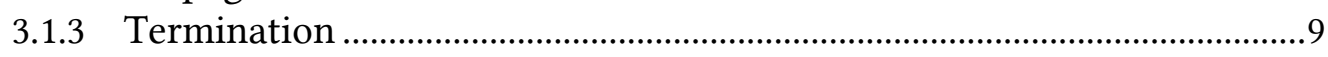

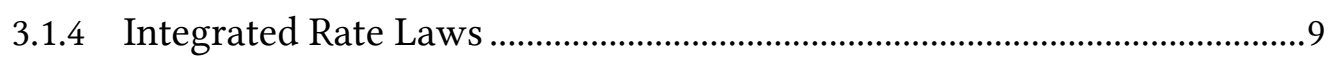

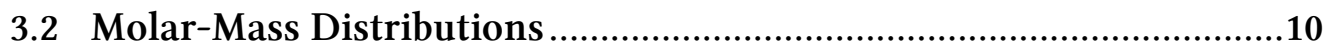

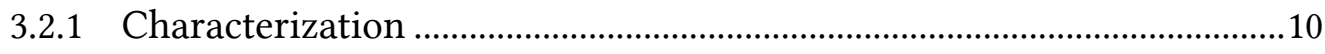

3.2.2 Determination via Size-Exclusion Chromatography...............................11

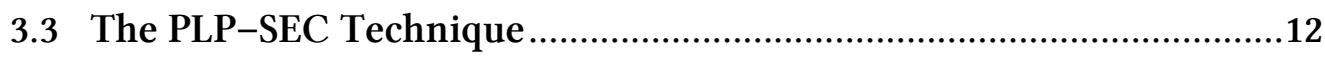

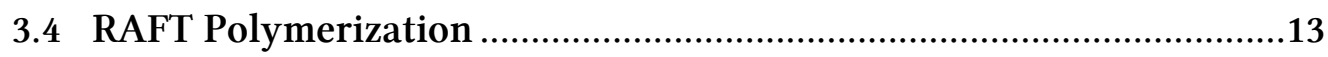

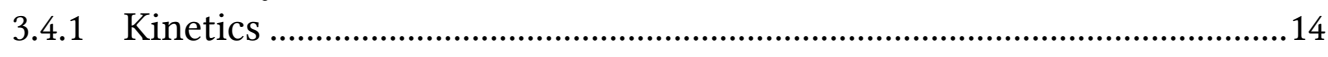

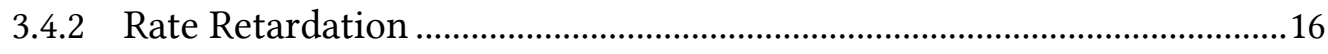

3.5 Surface-Initiated Polymerizations .................................................17

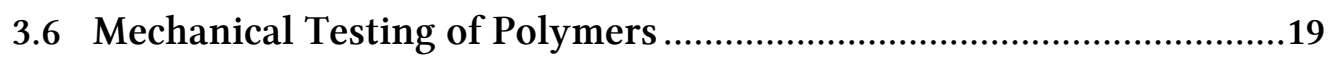

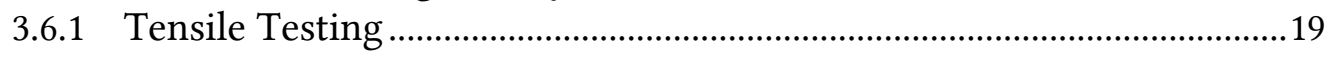

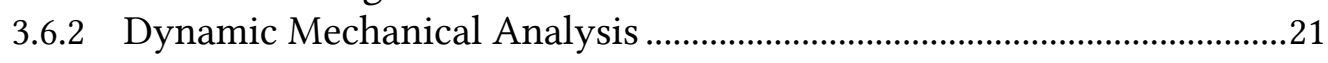

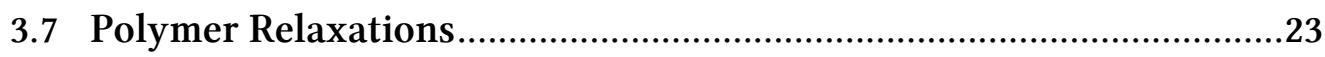

PART 1: Surface-Initiated Polymerizations ............................. 25

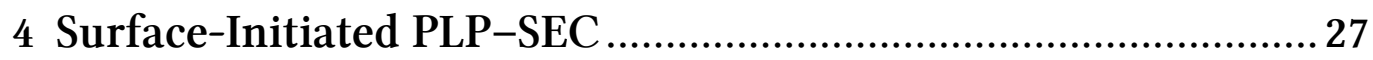

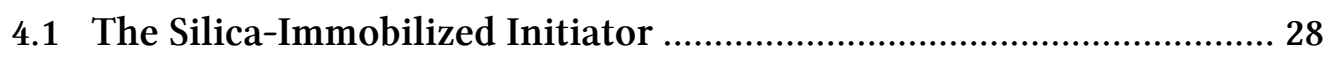

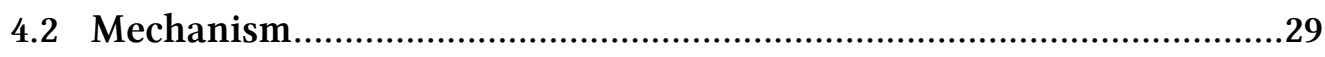

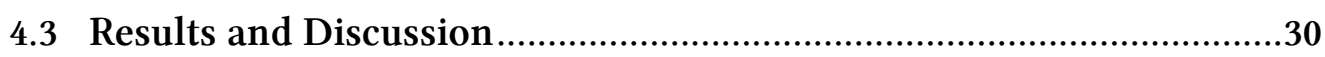

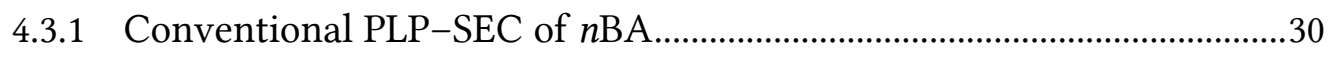




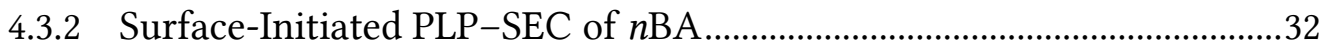

4.3.3 Surface-Initiated PLP-SEC of Styrene .........................................................

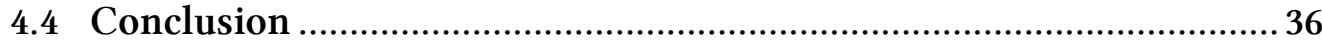

5 Surface-Initiated Polymerization of Styrene ...............................39

5.1 Surface-Initiated Conventional Radical Polymerization ....................40

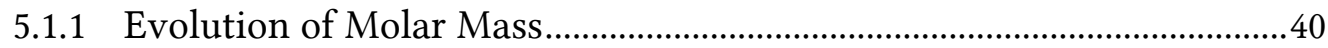

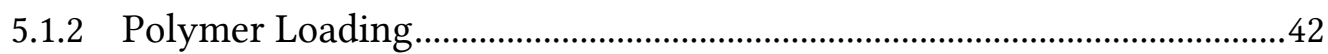

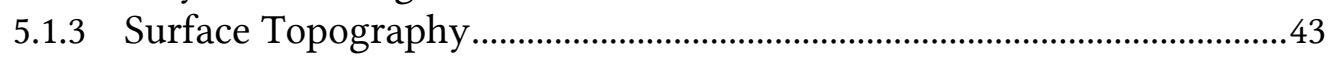

5.2 Surface-Initiated RAFT Polymerization............................................. 48

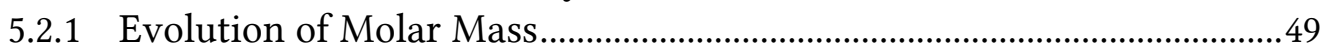

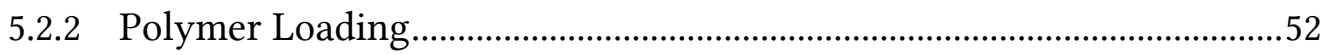

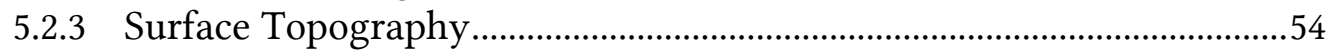

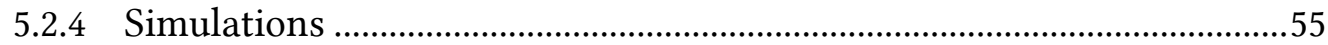

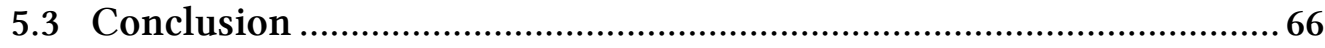

Part 2: Mechanical Properties of Polymer Materials ...............69

6 Tensile Properties of Silica-Filled Styrene-nBA Copolymers ...71

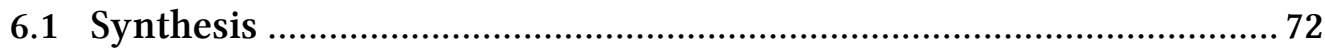

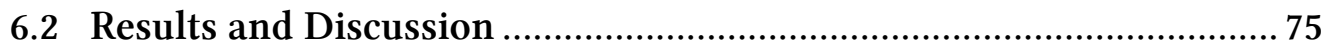

6.2.1 Impact of Silica Content...............................................................................

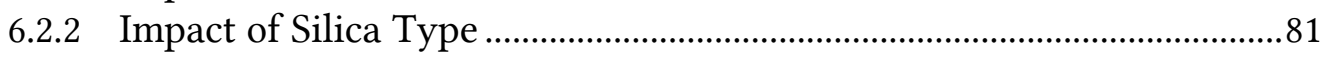

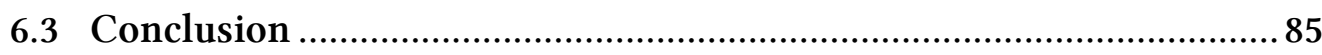

7 Relaxation Processes of ABA Type $t$ BA-AA Copolymers........87

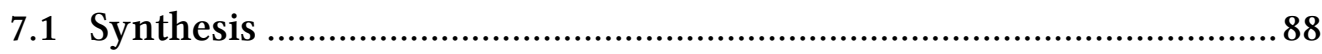

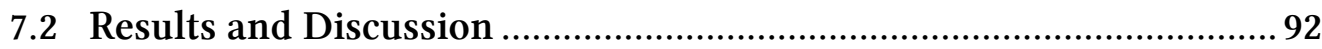

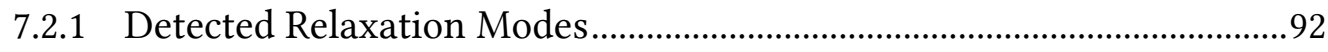

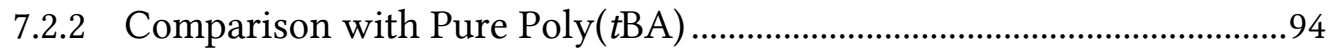

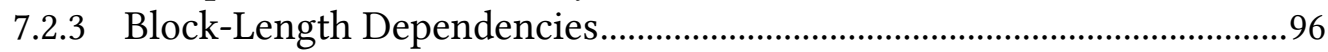

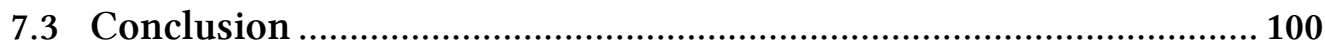

8 Tensile Properties of MA-AA Copolymers ........................... 103 


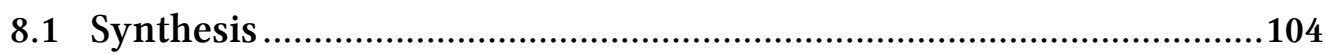

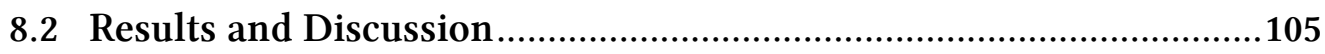

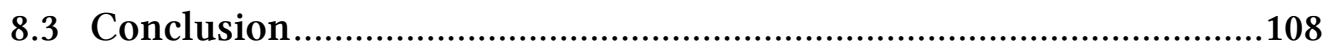

9 Closing Remarks ................................................................. 111

10 Experimental Part............................................................ 117

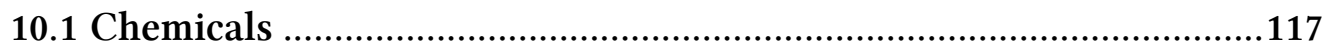

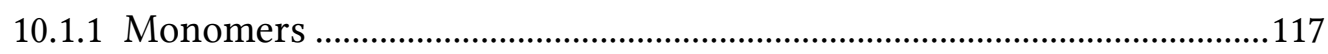

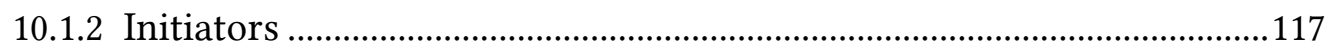

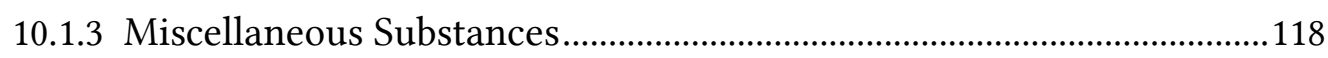

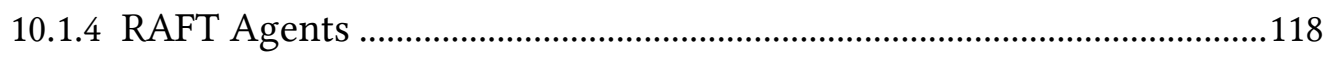

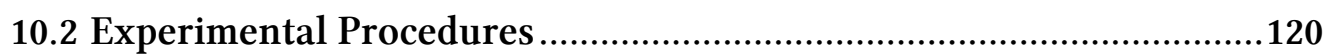

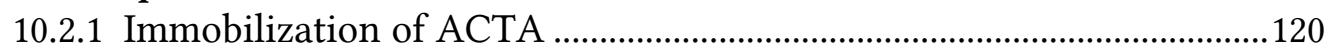

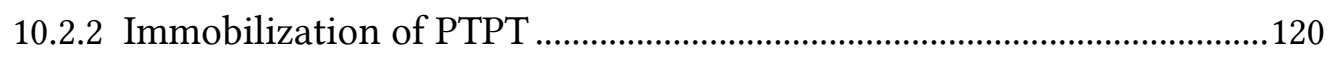

10.2.3 Pulsed-Laser Polymerizations (PLP) _.....................................................120

10.2.4 Surface-Initiated Conventional Radical Polymerization of Styrene ...121

10.2.5 Surface-Initiated RAFT Polymerization of Styrene .................................121

10.2.6 Cleavage of Silica-Bound Polymer ...............................................................122

10.2.7 Synthesis of Silica-Filled Styrene- $n$ BA Copolymers................................122

10.2.8 Extraction of Pure Styrene- $n$ BA Copolymers ..........................................122

10.2.9 Synthesis of ABA Type $t \mathrm{BA}-\mathrm{AA}$ Copolymers ….....................................122

10.2.10 Synthesis of Random MA-AA Copolymers............................................123

10.2.11 Synthesis of the ABA Type MA-AA Copolymer ..................................123

10.2.12 Sample Preparation for Tensile Analysis................................................124

10.2.13 Sample Preparation for DMA..................................................................124

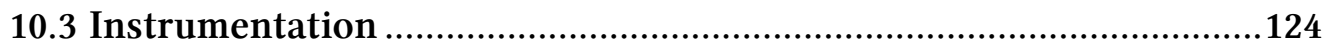

10.3.1 Size-Exclusion Chromatography ………................................................124

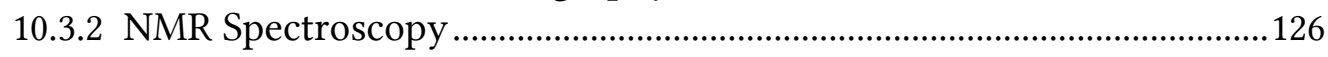

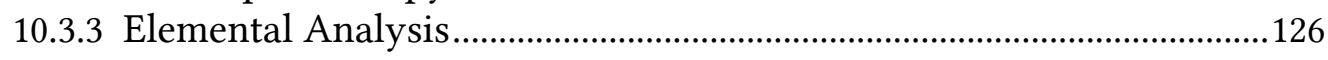

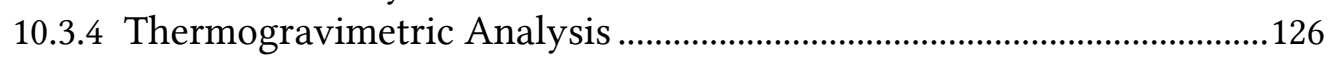

10.3.5 Scanning Electron Microscopy …………...................................................126

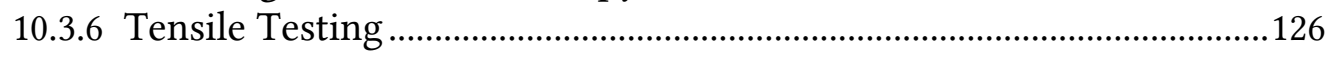

10.3.7 Dynamic Mechanical Analysis …………..................................................127

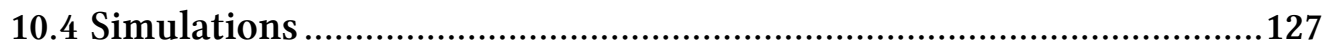

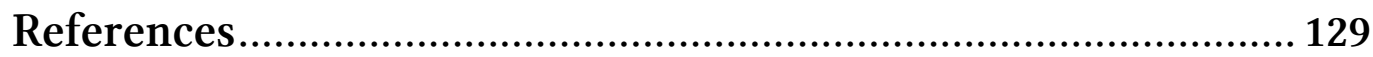


Appendices

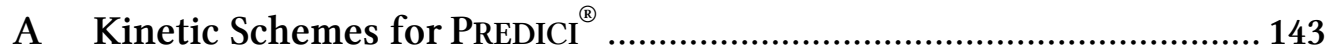

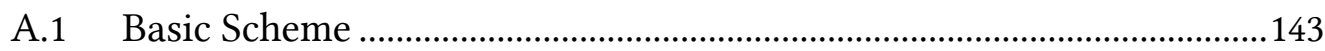

A.2 Extended Scheme for the IRT Model.......................................................144

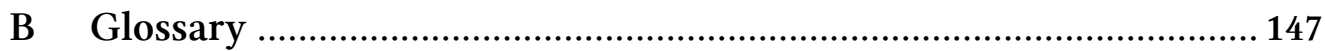




\section{Abstract}

In this work, kinetic and mechanistic aspects of the radical polymerization from the surface of fumed silica particles were examined. The surface-initiated polymerization technique was complemented by reversible additionfragmentation chain transfer (RAFT) polymerization in order to synthesize silica-polymer composites and ABA type copolymers containing hydrogen bonds for mechanical analysis.

An approach for the determination of propagation rate coefficients, $k_{\mathrm{p}}$, of surface-initiated radical polymerizations is presented. The main feature of this approach is the application of a silica-immobilized photoinitiator in pulsedlaser polymerization-size-exclusion chromatography (PLP-SEC). The determined $k_{\mathrm{p}}$ values for styrene and $n$-butyl acrylate $(n \mathrm{BA})$ are noticeably higher compared with the International Union of Pure and Applied Chemistry (IUPAC) benchmark data for polymerizations in solution.

The surface-initiated radical polymerization of styrene triggered by thermal decomposition of the silica-anchored azo initiator was examined for both the conventional and the RAFT approach. The conventional radical surfaceinitiated polymerization of styrene yielded grafted polystyrene whose molar masses were independent of total monomer conversion. The loading of grafted polymer on the silica surface increased steadily during the course of polymerization and reached a maximum value of $48 \%$. This specific sample of maximum polymer loading further exhibited several eye-catching sites in scanning electron microscopy (SEM), where the anchored polystyrene seemed to be visible as filaments of $1 \mu \mathrm{m}$ length at the site of cracks in the surface.

The addition of RAFT agent to the interstitial solution induces living behavior for both the initiator-derived chains on the surface and the RAFTderived chains in solution. As a result, the molar masses increased linearly towards higher conversion and polymers with narrow molar-mass distributions (MMDs) were obtained. The MMDs of the anchored polystyrene were slightly broader than the MMDs of the free polystyrene and additionally displayed a high-molar-mass shoulder. This shoulder was identified in PREDICI ${ }^{\circledR}$ (Polyreac- 


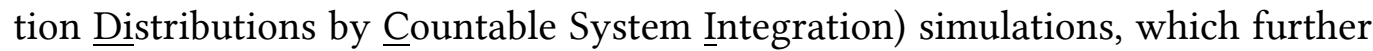
indicate a reduced addition rate coefficient of the main equilibrium between free and grafted species.

The surface-initiated RAFT polymerization was used to synthesize silicafilled styrene- $n \mathrm{BA}$ copolymers in a one-step procedure. The resulting composites were tailored with respect to the molar mass of the copolymer, its monomeric composition, the silica content and the loading of anchored copolymer on the corresponding silica particles. The latter was achieved by the addition of pure, initiator-modified and RAFT agent-modified silica particles, respectively, to the polymerization system. This approach allowed for tuning the amount of surface-grafted copolymer on the silica surface that was formed in situ during polymerization. Tensile testing was used to evaluate the mechanical properties of the composite materials. The silica content and the surface modification of the silica particles display crucial composite properties that largely affected the tensile performance.

Copolymers, in which hydrogen bonds were introduced in a controlled manner, were synthesized to systematically study a recently detected secondary relaxation mode at temperatures below the glass transition. This was achieved by RAFT polymerization of the two monomers tert-butyl acrylate $(t \mathrm{BA})$ and acrylic acid (AA) to yield ABA type copolymers, in which the inner B-block consists of pure poly $(t \mathrm{BA})$. The two outer A-blocks, on the other hand, contain a mixture of $t \mathrm{BA}$ and $\mathrm{AA}$ and can thus form hydrogen bonds. Dynamic mechanical analysis (DMA) revealed the occurrence of this secondary relaxation mode termed "chemical confinement" (cc). The glass transition temperature was thoroughly examined as well as a high-temperature relaxation that is absent in pure poly $(\mathrm{BA})$. This relaxation at high temperatures was further accompanied by a distinctive flow behavior of the copolymers in the rubbery region.

The impact of the AA content and the AA location in the polymer chain on the tensile properties was probed for copolymers of methyl acrylate (MA) and AA. The AA content largely affected the tensile properties of the copolymers. The insertion of AA into the outer chain parts of poly(MA), which resembles the ABA type arrangement of the $t \mathrm{BA}-\mathrm{AA}$ copolymers, yielded a copolymer with enhanced tensile modulus and tensile strength compared with random MA-AA copolymers. 


\section{Introduction}

I was trying to make something really hard, but then I thought I should make something really soft instead, that could be molded into different shapes. That was how I came up with the first plastic. I called it Bakelite.

(Leo Hendrik Baekeland)

The invention of the first man-made synthetic plastic named "Bakelite" by L. H. Baekeland in 1907 marks the advent of commercial polymer materials. ${ }^{[1]}$ However, the pioneering works by Staudinger et al. in the 1920's truly formed the basis for modern macromolecular science. ${ }^{[2]}$ Since then, persistent research has been conducted on this field to arrive at tailored materials for a wide range of applications. Nowadays, synthetic polymers are used in domains such as packaging, sports and outdoor equipment, paints, sealants, electronics, automobiles, as well as in the aircraft and space industry. ${ }^{[3]}$ In Germany, the amount of produced plastics exceeded 12 million tons in 2010 with a corresponding annual turnover of 51.3 billion $€ .^{[4]}$ The continuing growth of polymer applications facilitates economic growth and prosperity, and today's life can hardly be imagined without the use of commercial plastic materials.

Polymers can be synthesized by for example polycondensation and catalytic, ionic, or radical polymerization. Especially radical polymerization is extensively used in industry due to its robustness and versatility towards monomers and reaction conditions. ${ }^{[5]}$ The addition of mediating reagents to a radicalpolymerization system further enables control over molar mass and macromolecular architecture similar to living ionic polymerizations. Among such techniques, particularly RAFT polymerization has attracted increasing attention since its invention in $1998,{ }^{[6]}$ because all above-mentioned strengths of radical polymerization are retained. The ability to tailor the macromolecular architecture is of tremendous importance to meet the increasing requirements of plastic materials for special applications. This is because the macroscopic material properties of a polymer are governed by its molecular structure (Figure 2-1). The polymer structure, in turn, is determined by the multitude of 
individual reactions that occur in radical polymerization. ${ }^{[7,8]}$ For this reason, a detailed understanding is required that bridges polymerization kinetics, macromolecular architecture, and the material properties of the resulting polymer.

\section{Macromolecular Architecture}
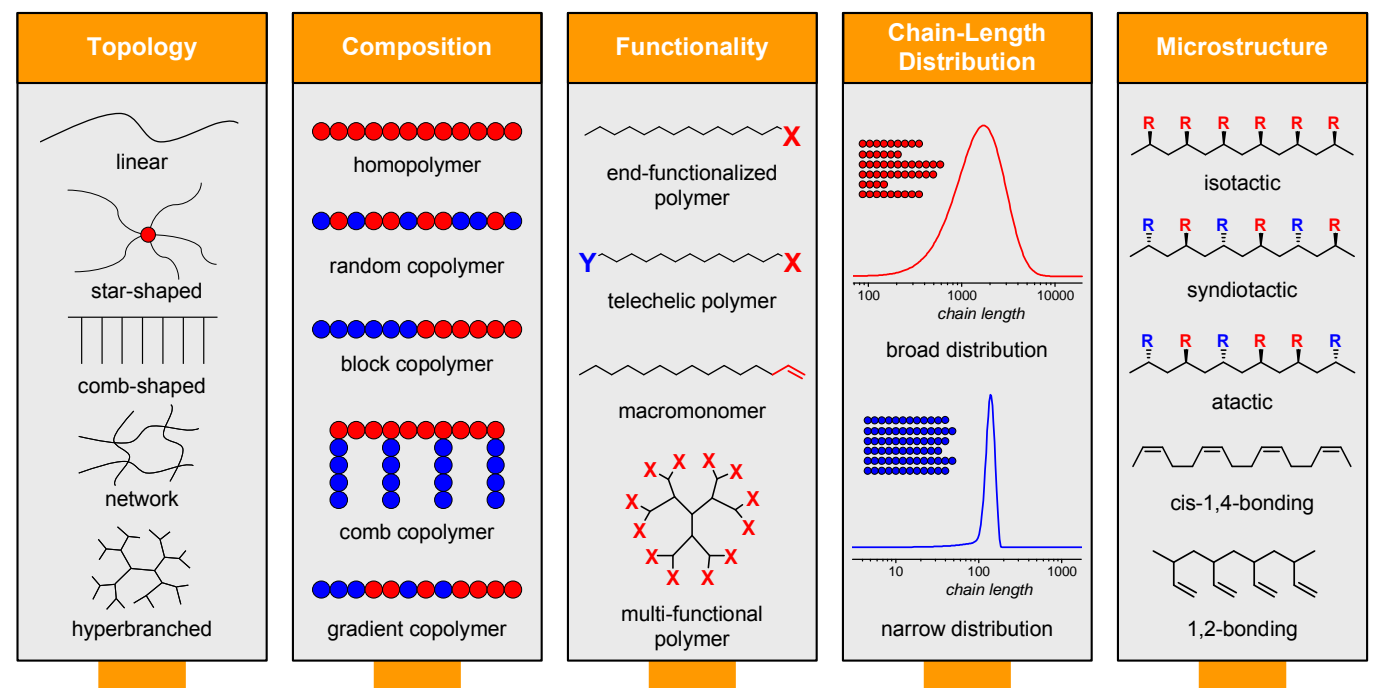

\section{Polymer Properties}

Figure 2-1. Overview of different structural attributes that contribute to polymer properties.

A field that has opened up remarkable opportunities for application of polymers is the one of surface-initiated radical polymerizations. In this approach, macroradicals grow from a solid support and thus form covalently anchored polymer layers. The variety of accessible solid substrates is extremely large and can be tailored not only with respect to its chemical composition but also in terms of its shape, which-in combination with the large number of surface-attachable polymers-results in an almost infinite variability of achievable materials. So far, polymers have been successfully grafted onto a multitude of substrates including silica particles, ${ }^{[9-11]}$ carbon black, ${ }^{[12]}$ silver $^{[13]}$ gold ${ }^{[14-16]}$ silica wafers, ${ }^{[17-20]}$ carbon nanotubes ${ }^{[21-23]}$ and nanorods, ${ }^{[24]}$ cellulose ${ }^{[25,26]}$ as well as polymers itself. ${ }^{[27-29]}$ In particular, the modification of inorganic particles with organic polymer layers has aroused increasing interest in recent years due to the tunable and unique properties of the resulting hybrid materials. Current applications of such materials include adhesives, coatings, biomaterials, and microelectronics. ${ }^{[30-32]}$ The kinetics of surface-initiated radical 
polymerizations is, however, not easily accessible and investigations are thus scarce, despite the fact that the polymerization kinetics governs the properties of the final polymer film to a large extent. Rühe et al. showed that the overall propagation rate on the surface is similar to polymerization in solution. ${ }^{[33]}$ However, state-of-the-art techniques, as for instance PLP-SEC, have not yet been applied to surface-initiated radical polymerizations. Hence individual rate coefficients, such as the propagation rate coefficient, are still lacking for this promising type of radical polymerization.

Another important aspect for radical polymerizations from surfaces is the interplay of the molar masses of fixed and free polymer, which in most cases form simultaneously during surface-initiated polymerizations. ${ }^{[3,35]}$ The MMDs for both types of polymer display an essential attribute, as they do not only allow insight into the kinetics during polymerization, but also affect important parameters of the polymer film such as its thickness. However, exact control and interpretation of the MMDs often remain an obstacle. ${ }^{[3]}$ This circumstance could be tackled by PREDICI ${ }^{\circledR}$ simulations,${ }^{[37]}$ which have proven to be a powerful tool for probing the impact of kinetic coefficients on the molar-mass control of radical polymerizations. ${ }^{[38]}$

Recently, the surface topography of poly(MA)-modified silica particles, determined by means of SEM, exhibited substantial effects caused by variations of the molar mass and loading of the anchored polymer ${ }^{[35,39]}$ The surface of the silica-polymer composites displayed a more structured and smooth character upon polymer grafting compared with pure silica particles. However, the sample amount was limited and thus allowed only a rough interpretation of the obtained SE (scanning electron) micrographs.

Grafting polymer from a surface not only allows for producing tailored polymer films, but it may also permit the in-situ synthesis of polymers filled with inorganic particles. This approach may benefit from the modification of the filler particles with polymer chains during polymerization, which assures compatibility between the inorganic particles and the matrix polymer. This issue is highly relevant to achieve the desired properties of composite materials, as for example silica-polymer nanocomposites. ${ }^{[40]}$ This class of composites is subject of extensive research, as the resulting silica-filled polymers often display enhanced mechanical properties and thermal stability compared with their unfilled analogues. Particularly, tensile testing displays one of the best suited methods for characterizing nanocomposite materials, yielding important parameters such as the Young's modulus, tensile strength, and elongation at break ${ }^{[40]}$ However, in spite of the large extent of research work on this domain, the interpretation of the determined material properties remains complex. So far, no universal pattern exists that describes the influence of the structural features on the macroscopic properties of silica-polymer nanocomposites. 
The establishment of structure-property relationships also remains a challenge for conventional synthetic polymers. Among other things, this can be attributed to the large amount of parameters that affect, for instance, the mechanical properties of a plastic specimen in tensile analysis. ${ }^{[41]}$ In addition to that, the structure of the polymer, such as its composition, topology, and molar mass, not only has to be accurately known, but it also needs to be controlled in order to obtain results that can be systematically assigned to a certain molecular feature. Such a control over macromolecular architecture can be achieved by application of RAFT polymerization, which enables the synthesis of narrowly dispersed macromolecules of defined molar mass and with complex molecular structure, such as block copolymers and star polymers. ${ }^{[42]}$ By tailoring the polymer architecture, it is even possible to synthesize polymers that mimic certain structural features, such as the presence of pure polyethene segments in copolymers of ethene and methacrylic acid (MAA) with a non-random distribution of the two monomers. ${ }^{[43]}$ In these copolymers, a novel relaxation mode below their glass transition temperature was detected by DMA, which represents a powerful technique for the determination of relaxation modes. ${ }^{[4-48]}$ This novel relaxation mode was suggested to originate from a chemical confinement (cc) of the ethene segments in between MAA segments. However, the composition of these copolymers could not be entirely determined. Moreover, it is uncertain if the proposed concept of chemical confinement can be generally applied to polymers. By combination of RAFT polymerization and mechanical spectroscopy, changes in the relaxation spectra produced by certain structural features may be systematically assessed. Such relaxation processes display an essential attribute, as they control the macroscopic mechanical behavior. ${ }^{[49-51]}$ Hence, the detailed analysis of these relaxation processes and their origin on a molecular scale are of high interest for producing advanced plastic materials. Additionally, these relaxations also feature substantial impact on the tensile properties of polymers, which has been shown for polyvinyl chloride. ${ }^{[52]}$ Therefore, a complementary application of the two mechanical testing techniques DMA and tensile analysis should prove advantageous.

One primary objective of this work is the investigation of kinetic and mechanistic aspects of surface-initiated (RAFT) polymerizations by application of PLP, SEC, thermogravimetric analysis (TGA) and PREDICI ${ }^{\circledR}$ simulations. Another main objective is the usage of RAFT and surface-initiated polymerization to synthesize silica-filled polymers as well as copolymers, in which hydrogen bonds are introduced in a controlled fashion. Since the introduction of filler particles and hydrogen bonds, respectively, may largely affect the mechanical properties of polymers, these materials are to be subjected to DMA and tensile testing in order to obtain structure-property relationships. 


\section{Theoretical Background}

The aim of this chapter is to provide the reader with a selection of theoretical background information, which forms the basis of the research that is performed in this thesis. It starts by briefly describing the ideal reaction scheme for radical polymerization, before the characterization and determination of MMDs is examined. The PLP-SEC technique, which is the method of choice for determining the propagation rate coefficient, $k_{\mathrm{p}}$, in radical polymerization, is then presented. Surface-initiated polymerizations, which display an interesting system from both a kinetic and a synthetic point of view, are also highlighted here. Subsequently, the RAFT process, which constitutes a powerful type of controlled radical polymerization, is covered in detail. Finally, the mechanical testing of polymers via tensile experiments and DMA as well as relaxations in polymers are outlined.

\subsection{Ideal Polymerization Kinetics}

Ideal polymerization kinetics is grounded on four assumptions: ${ }^{[53]}$

- All reactions are irreversible.

- Monomeric species are exclusively consumed in propagation steps.

- All macroradicals show the same reactivity, regardless of their chain length.

- Termination proceeds only by disproportionation or by bimolecular radical combination.

Based on these assumptions a kinetic scheme of conventional radical polymerization can be characterized by three fundamental reaction steps: the formation of radicals in the initiation reaction, chain growth of these radicals by propagation, and termination of radical chains. ${ }^{[53]}$ 


\subsubsection{Initiation}

The formation of radicals can take place by for example thermal, chemical or photochemical excitation of an initiator or by direct excitation of the monomer: $:^{[53]}$

$$
\mathrm{I} \stackrel{k_{\mathrm{d}}}{\longrightarrow} 2 \mathrm{R}^{\bullet} .
$$

The rate of radical formation for thermally excited polymerizations is given by a first-order kinetic law:

$$
\frac{\mathrm{d} c_{\mathrm{R}}}{\mathrm{d} t}=2 \cdot k_{\mathrm{d}} \cdot f \cdot c_{\mathrm{I}}
$$

where $c_{\mathrm{R}}$ and $c_{\mathrm{I}}$ are the radical and initiator concentration, respectively, $k_{\mathrm{d}}$ is the initiator decomposition rate coefficient and $f$ denotes the initiator efficiency, that is the fraction of initiator-derived radicals that actually start chain growth.

In case of photochemical initiation induced by short ultraviolet (UV) laser pulses (with a typical pulse width of $20 \mathrm{~ns}$ for the lasers used in this work) applied to the reaction mixture containing monomer and initiator (PLP), the laser pulse instantaneously creates a significant amount of primary radicals. The formation of radicals is fast in comparison to a subsequent termination or propagation step. The primary radical concentration, $c_{\mathrm{R}}^{0}$, which is generated by a single laser pulse, is given by:

$$
c_{\mathrm{R}}^{0}=2 \cdot \Phi \cdot \frac{n_{\mathrm{abs}}}{V}
$$

where $\Phi$ is the primary quantum yield, $n_{\text {abs }}$ denotes the number of absorbed photons and $V$ is the irradiated volume. ${ }^{[33]}$

\subsubsection{Propagation}

In the propagation step the radicals grow by adding monomer molecules:

$$
\mathrm{R}_{i}^{\bullet}+\mathrm{M} \stackrel{k_{\mathrm{p}}}{\longrightarrow} \mathrm{R}_{i+1}^{\bullet} .
$$

The rate of propagation, that is the change in monomer concentration, $c_{\mathrm{M}}$, is given by the following rate law: ${ }^{[53]}$ 


$$
\frac{\mathrm{d} c_{\mathrm{M}}}{\mathrm{d} t}=-k_{\mathrm{p}} \cdot c_{\mathrm{M}} \cdot c_{\mathrm{R}}
$$

The propagation rate coefficient $k_{\mathrm{p}}$ is assumed to be independent of radical chain length as well as monomer conversion under ideal conditions.

\subsubsection{Termination}

The termination reaction takes place either by disproportionation, in which a $\beta$ hydrogen atom is transferred from one radical to another, or by combination, which is mostly a simple head-to-head coupling of two radicals: ${ }^{[53]}$

$$
\begin{gathered}
\mathrm{R}_{i}^{\bullet}+\mathrm{R}_{j}^{\bullet} \stackrel{k_{\mathrm{t}, \mathrm{d}}}{\longrightarrow} \mathrm{P}_{i}+\mathrm{P}_{j}, \\
\mathrm{R}_{i}^{\bullet}+\mathrm{R}_{j}^{\bullet} \stackrel{k_{\mathrm{t}, \mathrm{c}}}{\longrightarrow} \mathrm{P}_{i+j} .
\end{gathered}
$$

The termination rate coefficient, $k_{\mathrm{t}}$, is the sum of the individual rate coefficients for disproportionation, $k_{\mathrm{t}, \mathrm{d}}$, and combination, $k_{\mathrm{t}, \mathrm{c}}$. The termination reaction, in which the actual "dead" polymer is formed, is preceded by the translational diffusion of the two radicals and the segmental diffusion to make contact between their radical sites. Both preliminary steps are implemented in the termination rate coefficient. The overall rate of termination is of second order with respect to the radical concentration $c_{\mathrm{R}}:^{[53]}$

$$
\frac{\mathrm{d} c_{\mathrm{R}}}{\mathrm{d} t}=-2 \cdot k_{\mathrm{t}} \cdot c_{\mathrm{R}}^{2}
$$

\subsubsection{Integrated Rate Laws}

In case of stationary radical polymerization, the rates of radical formation via initiator decomposition (Equation 3-1) and of radical loss by termination (Equation 3-4) are equal: ${ }^{[54]}$

$$
2 \cdot k_{\mathrm{d}} \cdot f \cdot c_{\mathrm{I}}=2 \cdot k_{\mathrm{t}} \cdot c_{\mathrm{R}}^{2} .
$$

The expression for the overall rate of polymerization, $R_{\mathrm{p}}$, in an ideal stationary radical polymerization is obtained by combination of equations $3-3$ and $3-5::^{[54]}$

$$
R_{\mathrm{p}}=-\frac{\mathrm{d} c_{\mathrm{M}}}{\mathrm{d} t}=c_{\mathrm{M}} \cdot \frac{k_{\mathrm{p}}}{\sqrt{k_{\mathrm{t}}}} \cdot \sqrt{k_{\mathrm{d}} \cdot f \cdot c_{\mathrm{I}}} .
$$




\subsection{Molar-Mass Distributions}

All synthetic polymers exhibit a molar-mass distribution (MMD) caused by the inherent statistics contained in the reactions steps during their synthesis. The determination of these mass distributions is essential, as they allow insight into the reaction kinetics during polymerization and further affect the macroscopic properties of a polymer material.

\subsubsection{Characterization}

Important parameters of MMDs include the average molar masses. The number-average molar mass, $M_{\mathrm{n}}$, considers the numeric abundance of each chain length $i$ in the polymer:

$$
M_{\mathrm{n}}=\sum_{i=1}^{\infty} x_{i} M_{i}=\frac{\sum_{i=1}^{\infty} N_{i} M_{i}}{\sum_{i=1}^{\infty} N_{i}},
$$

where $M_{i}$ is the molar mass of a macromolecule with chain length $i . x_{i}$ and $N_{i}$ denote the amount fraction and number of molecules with chain length $i$, respectively. The weight average molar mass, $M_{\mathrm{w}}$, accounts for the weight fractions $w_{i}$ of all macromolecules:

$$
M_{\mathrm{w}}=\sum_{i=1}^{\infty} w_{i} M_{i}=\frac{\sum_{i=1}^{\infty} N_{i} M_{i}^{2}}{\sum_{i=1}^{\infty} N_{i} M_{i}} .
$$

The ratio of these average molar masses yields the polydispersity index, PDI, which is a measure of the broadness of MMDs:

$$
\mathrm{PDI}=\frac{M_{\mathrm{w}}}{M_{\mathrm{n}}} \geq 1 .
$$

The lowest possible PDI values under ideal conditions in conventional radical polymerization are 2.0 for termination via disproportionation and 1.5 for termination via combination, respectively. ${ }^{[5]}$

In case of (pseudo-)living polymerizations, for example reversibledeactivation radical polymerization, ${ }^{[5]}$ popularly known as living/controlled 
radical polymerization, ${ }^{[56]}$ the chain length distribution reflects a Poisson distribution and the PDI can then be expressed as a function of the numberaverage degree of polymerization $P_{\mathrm{n}}$ :

$$
\mathrm{PDI}=1+\frac{1}{P_{\mathrm{n}}}
$$

The PDI hence approaches values close to unity under ideal molar-mass control and is in that case well below the minimum of 1.5 for conventional radical polymerizations. As a conclusion, the dispersity of a polymer can serve as a quality criterion for the molar-mass control of a controlled radical polymerization.

\subsubsection{Determination via Size-Exclusion Chromatography}

SEC constitutes the most widely applied method for the fast determination of MMDs. ${ }^{[41]}$ In this method, dilute solutions of polymers are passed through columns with highly porous filler materials (e.g. swollen crosslinked polymer) at constant flow rate. The macromolecules are separated by their hydrodynamic volume, $V_{\mathrm{h}}$, which correlates with their molar mass $M$ through the intrinsic viscosity $[\eta]$ :

$$
V_{\mathrm{h}}=[\eta] \cdot M \text {. }
$$

In an SEC experiment, the hydrodynamic volume of a macromolecule is inversely proportional to its elution time and elution volume, $V_{\mathrm{e}}$, which is the volume of mobile phase passed through between the injection point and the peak maximum. This arises from the fact that smaller polymer coils are able to penetrate a larger number of pores and are thus eluted later.

After their separation the polymer chains are detected by, for example, changes in refractive index (RI) or UV absorption. The retention times are, however, strongly depending on the experimental setup (polymer type, solvent, temperature, column filler), which is why a calibration procedure is required for obtaining molar masses. The distributions obtained from SEC are mass MMDs on a logarithmic scale. ${ }^{[57]}$

In case calibration standards are not available for a certain type of polymer its MMD may be estimated via the principle of universal calibration ${ }^{[58]}$ At a given elution volume, two polymers 1 and 2 are assumed to have the same hydrodynamic volume: ${ }^{[59]}$

$$
[\eta]_{1} \cdot M_{1}=[\eta]_{2} \cdot M_{2} .
$$


A commonly used expression for the correlation between intrinsic viscosity and molar mass is given by the Mark-Houwink $(\mathrm{MH})$ equation: ${ }^{[60,61]}$

$$
[\eta]=K \cdot M^{a},
$$

where $K$ and $a$ are the so-called $\mathrm{MH}$ parameters. These parameters are known for a large number of polymers and may be used for universal calibration. From the above two equations the following relation can be derived:

$$
\log M_{2}=\frac{1}{1+a_{2}} \log \frac{K_{1}}{K_{2}}+\frac{1+a_{1}}{1+a_{2}} \log M_{1}
$$

with the indices 1 and 2 reflecting the polymer used for calibration and the polymer of interest, respectively. If all $\mathrm{MH}$ parameters are known the molar mass of the analyzed polymer can be easily determined from SEC data. If this is not the case, the calibration can be circumvented by application of molar masssensitive detectors such as viscometer ${ }^{[62]}$ or light scattering ${ }^{[63]}$ type detectors.

\subsection{The PLP-SEC Technique}

PLP in conjunction with subsequent analysis of the formed polymer via SEC has established itself as the method of choice for determining individual $k_{\mathrm{p}}$ values in radical polymerization. ${ }^{[64]}$

In a typical PLP-SEC experiment, a mixture of monomer and photoinitiator is illuminated at pre-selected temperature by short laser pulses (approximately $20 \mathrm{~ns}$ long) separated by a time of $t_{0}$, which is determined by the laser frequency. Each laser pulse almost instantly produces photoinitiator-derived radicals that start chain growth by adding monomer molecules. Although termination occurs at any time during the experiment, the high population of radicals at each laser pulse induces preferential chain stopping points for the propagating macroradicals. Therefore a considerable fraction of these macroradicals is terminated instantly at $t_{0}$, when the next laser pulse creates a new population of short radicals. These dead chains exhibit a chain length $L_{0}$, which is related to the dark time period $t_{0}$ in between two laser pulses via the following equation:

$$
L_{0}=k_{\mathrm{p}} \cdot c_{\mathrm{M}} \cdot t_{0}
$$

As not all radicals are terminated by the first laser flash but may grow until subsequent pulses, a well-structured MMD with peaks at multiples of $L_{0}$ is obtained (Figure 3-1). The chain length $L_{0}$, which is generally determined via 
the point of inflection on the low molar-mass side, ${ }^{[65,66]}$ directly yields the propagation rate coefficient via equation 3-15.

The existence of further inflection points at integer multiples of $L_{0}$ is part of the PLP-SEC self-consistency check, which assures that the first inflection point does indeed reflect the fraction of polymer terminated after $t_{0} \cdot{ }^{[67]}$ Further consistency criteria are that $k_{\mathrm{p}}$ values are independent of initiator concentration, laser pulse energy, as well as laser pulse frequency. ${ }^{[67]}$

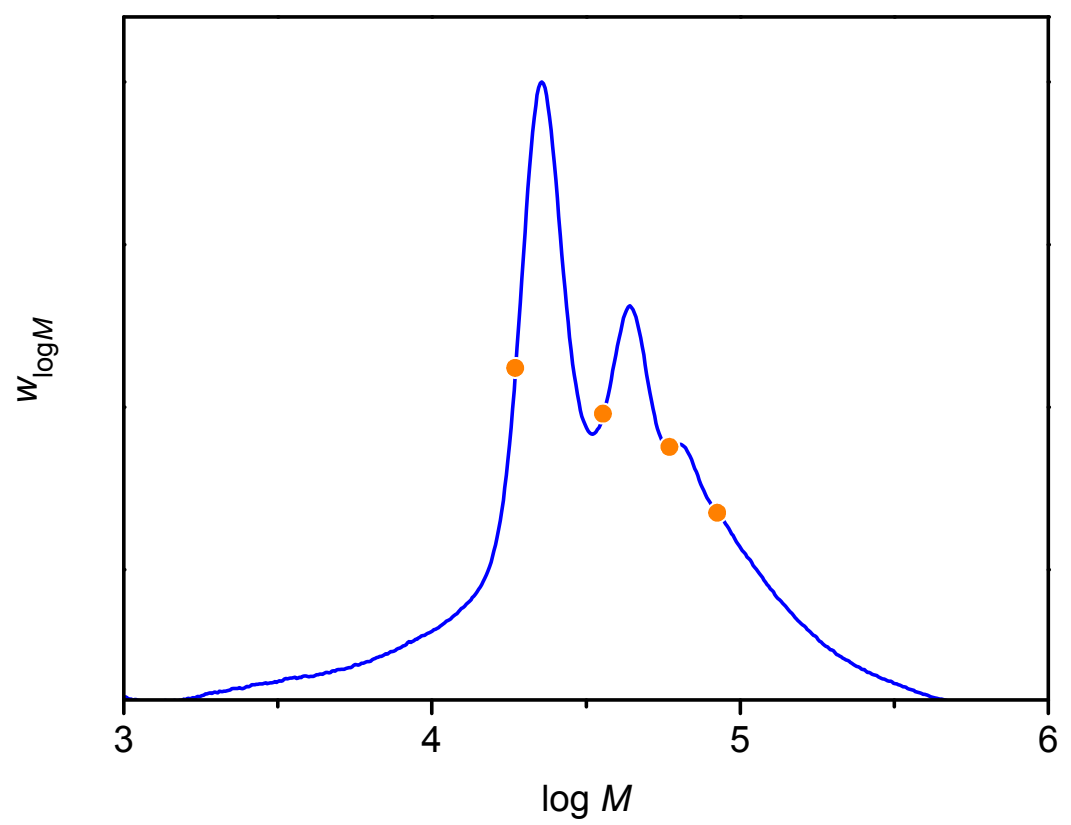

Figure 3-1. MMD (SEC trace) with indicated points of inflection from PLP of butyl methacrylate in bulk at $25^{\circ} \mathrm{C}$ and $20 \mathrm{~Hz}$ initiated by 2,2-dimethoxy-2-phenylacetophenone (DMPA) $\left(1.00 \mathrm{mmol} \cdot \mathrm{L}^{-1}\right)$.

\subsection{RAFT Polymerization}

Although conventional radical polymerization is one of the most widely used processes for the commercial production of high-molar-mass polymers, it is somewhat limited in terms of control over certain characteristics of the polymer such as molar mass, composition, and topology. ${ }^{[68]}$

The advent of controlled radical polymerization techniques since the mid-1990s has allowed for significant progress in this field as it enabled the design of tailored macromolecules in a relatively simple and straightforward manner. The techniques that are attracting greatest attention are nitroxidemediated polymerization $(\mathrm{NMP}),{ }^{[69]}$ atom transfer radical polymerization 
(ATRP), ${ }^{[70-72]}$ and RAFT polymerization. ${ }^{[6,73-78]}$ Especially the RAFT process, which was invented in 1998 by the Commonwealth Science \& Industrial Research Organization (CSIRO), has proven to be a very suitable technique for synthesizing polymers with complex architectures. ${ }^{[79,80]}$ RAFT polymerization is not only extremely versatile towards monomers and reaction conditions, ${ }^{[81-84]}$ but it also permits the predefinition of the macromolecular structure by applying specific RAFT agents that act as scaffolds for the desired polymer architecture. With this approach, various types of tailored polymer materials can be obtained with relative ease, like for example block copolymers, star and comb polymers. ${ }^{[79]}$ The detailed analysis of these polymers further allows for understanding the correlation of their mechanical properties and macromolecular architecture..$^{[85]}$

\subsubsection{Kinetics}

The RAFT mechanism is induced when a radical polymerization is carried out in the presence of thiocarbonylthio compounds (RAFT agents, generic formula in Figure 3-2) which react by reversible addition-fragmentation chain transfer. Apart from the active dithio moiety, RAFT agents generally feature the Z- and R-group, often termed activating and leaving group, respectively. ${ }^{[8,87]}$

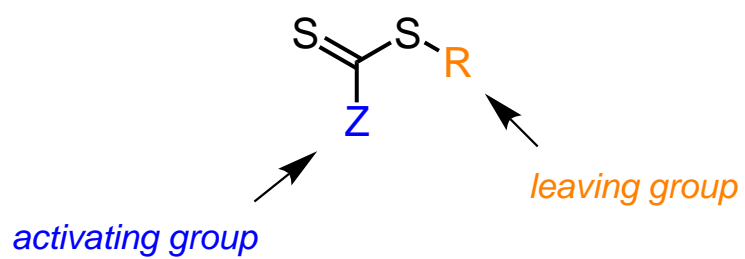

Figure 3-2. General chemical structure of RAFT agents.

The addition of RAFT agent to the reaction mixture of a conventional radical polymerization induces two addition-fragmentation equilibria that are superimposed on a conventional radical polymerization mechanism (Scheme 3-1).

At the beginning of polymerization, the radicals originally formed by the initiator react with the RAFT agent via the pre-equilibrium by adding to the carbon-sulfur double bond forming a radical intermediate. The Z-group of the RAFT agent is chemically designed in such a way that it activates the carbonsulfur double bond for radical attack and further stabilizes the radical intermediate. At this stage, the intermediate can either react back to the educts or proceed to release the R-group. As the pre-equilibrium is passed through 
quickly under ideal conditions, chain growth initiated by the R-group (with the reinitiation rate coefficient $k_{\mathrm{p} \text {,rein }}$ ) starts almost simultaneously for all chains, whose number is defined by the concentration of RAFT agent in the system. To achieve such conditions, the R-group should be designed as that its radical is stable relative to the monomeric radicals, allowing for fast fragmentation, while at the same time providing fast reinitiation, i.e. addition to monomer species.

Pre-equilibrium

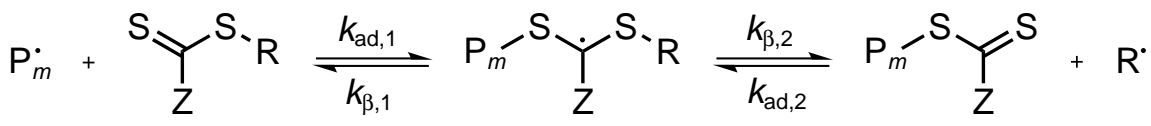

Reinitiation

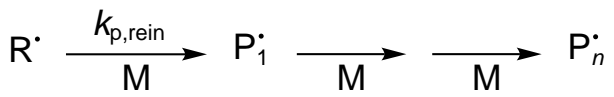

Main Equilibrium

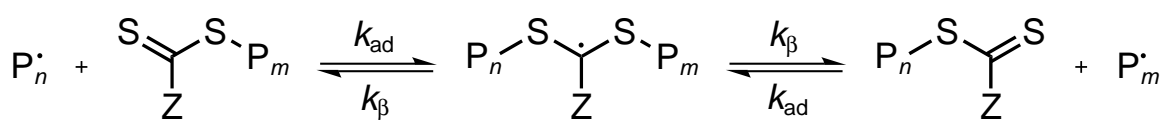

Scheme 3-1. Basic reaction steps in RAFT polymerization: pre-equilibrium, reinitiation and main equilibrium.

After all initial RAFT agent is consumed the polymerization is governed by the main equilibrium, which consists of the rapid exchange of active propagating radicals and dormant polymeric thiocarbonylthio compounds. This mechanism provides equal probability for all chains to grow and thus yields polymer with a narrow MMD. The molar mass increases linearly with increasing total monomer conversion, $X_{\mathrm{M}}$, and can be expressed by the following relation that assumes reactivity of all RAFT agent molecules and neglects the formation of initiator-derived radicals: ${ }^{[42]}$

$$
M_{\mathrm{n}}^{\text {theo }}=\frac{c_{\mathrm{M}} \cdot M_{\mathrm{M}} \cdot X_{\mathrm{M}}}{c_{\mathrm{RAFT}}}+M_{\mathrm{RAFT}} .
$$

Hence the theoretical number-average molar mass, $M_{\mathrm{n}}^{\text {theo }}$, can be controlled via the monomer concentration, monomer conversion, as well as the RAFT agent concentration, $c_{\mathrm{RAFT}}$, in the reaction mixture. $M_{\mathrm{M}}$ and $M_{\mathrm{RAFT}}$ denote the molar mass of monomer and RAFT agent, respectively. At the end of polymerization, the majority of chains retain the thiocarbonylthio end-group and can therefore 
be used for further (co)polymerization or other subsequent chemical reactions. ${ }^{[68,88-90]}$

The kinetic coefficients used to describe the rate of addition and fragmentation of the pre- and main equilibrium are $k_{\mathrm{ad}, 1}, k_{\beta, 1}, k_{\mathrm{ad}, 2}, k_{\beta, 2}$ and $k_{\mathrm{ad}}$, $k_{\beta}$, respectively. ${ }^{[91,92]}$ The main equilibrium is usually described by the equilibrium constant $K_{\text {eq }}{ }^{[93]}$ which is a measure for the stability of the intermediate radical:

$$
K_{\mathrm{eq}}=\frac{k_{\mathrm{ad}}}{k_{\beta}}
$$

\subsubsection{Rate Retardation}

In case the RAFT polymerization proceeds ideally, that is the chain-transfer process is fast and the RAFT intermediate is short-lived, the rate of polymerization should be similar to the one found for conventional radical polymerization because the radical concentration is not changed. However, some polymerization systems display a significant deceleration in polymerization rate (rate retardation), especially when dithiobenzoates are used as RAFT agents. ${ }^{[94]}$ This phenomenon may be explained by two fundamentally different mechanisms: ${ }^{[95]}$

\section{Slow fragmentation model}

The gist of this model, which is mainly supported by ab initio quantum chemical calculations, ${ }^{[96-100]}$ is a very small fragmentation rate of the intermediate radical in the main equilibrium compared with the rate of the addition reaction, yielding a high $K_{\text {eq }}$. The intermediate radical, whose stability is believed to be caused by delocalization of the unpaired electron on the phenyl ring that acts as Z-group in dithiobenzoates, may hence survive over a considerable period of time. Consequently, the propagating radical concentration and thus the rate of polymerization in the system would be effectively reduced. ${ }^{[101-103]}$ The proposed low $k_{\beta}$ values are, however, in conflict with experimental Electron Paramagnetic Resonance (EPR) data, since the slow fragmentation model predicts high intermediate radical concentrations that were not detected in such experiments. ${ }^{[104,105]}$ Moreover, fast decay of intermediate radicals has been observed via EPR after initiation by a single laser pulse. ${ }^{[106]}$

\section{Intermediate radical termination (IRT) model}

The intermediate radical may undergo irreversible termination either by crosstermination with a propagating radical or by self-termination with a radical of 
its own species. These side reactions would inherently lead to a reduction of radical concentration and thus also of polymerization rate. Although the possibility of such reactions has in principle been demonstrated in model experiments, ${ }^{[107,108]}$ the proposed termination products have not yet been isolated from dithiobenzoate-mediated polymerizations. This discrepancy may be explained by a very small concentration of IRT products that are needed to cause rate retardation and by assuming termination of merely oligomeric intermediate radicals, ${ }^{[109,110]}$ which would in both cases hinder the detection of such species via SEC.

\subsection{Surface-Initiated Polymerizations}

Radical polymerization from surfaces enables the in-situ synthesis of anchored polymer films. In pursuing this approach, often termed "grafting-from" approach, ${ }^{[111]}$ one can either immobilize a conventional radical initiator or a mediating agent (e.g. RAFT agent) to arrive at surface-anchored polymer (Figure 3-3). Alternatively, polymers may also be linked to the surface via functional end-groups, which is referred to as the "grafting-to" approach. ${ }^{[11]}$ This approach, however, suffers from significant drawbacks as the accessible grafting densities are limited because of steric repulsions between polymer chains. ${ }^{[11-114]}$

The application of anchored initiators for producing surface-tethered polymer has been used with great success as it enables the formation of considerable amounts of grafted polymer chains. ${ }^{[34,35,115-118]}$ By polymerizing in a conventional fashion, however, important properties of the fixed polymer such as chain length and topology are difficult to adjust, which can be partially overcome by adding a free RAFT agent to the solution ${ }^{[26,28,29,119-124]}$ or by simultaneously anchoring the initiator together with the RAFT agent. ${ }^{[35]}$ In this case the surface-grafted chains exhibit living behavior. ${ }^{[26,35]}$

To produce surface-confined polymer in a fully controlled manner via the "grafting-from" technique, the method of choice is to immobilize the mediating agent itself. ${ }^{[111]}$ This can in principle be performed with any controlled radical polymerization technique. However, RAFT polymerization from solid surfaces has proven to be extremely robust, versatile, and tolerant towards monomers, reaction conditions, and solid substrates. ${ }^{[20,125-131]}$ In addition to that, RAFT polymerization from solid surfaces allows two different approaches, as RAFT agents can be anchored either via their Z-group or R-group. Both the Z-group and the R-group approach have their own individual benefits and drawbacks depending on the experimental objective.

In case of the Z-group approach, ${ }^{[132-136]}$ the RAFT agent is irreversibly anchored to the solid substrate via its stabilizing group (Figure 3-3) and thus 
prevents chain-chain coupling of grafted growing macroradicals similar to Zgroup supported RAFT star polymerizations. ${ }^{[137]}$ In addition, this technique allows the easy separation of living grafted chains from nonliving chains occuring in solution, while at the same time it enables the regeneration of the anchored RAFT agent. However, the grafting density of the polymer is generally somewhat limited in case of the Z-group approach, as the reactive RAFT moieties are located close to the surface during the polymerization.

The inverse "R-group approach", ${ }^{[20,25,138,139]}$ where the RAFT agent is linked to the surface via its reinitiating group (Figure 3-3), does not suffer from this limitation, since the reactive centers grow away from the surface during chain growth and sterical hindrances are hence minimized. However, side reactions of the immobilized macroradicals such as chain transfer and termination become more probable. Hence a small fraction of tethered chains may not contain the RAFT end-group.

Recently an alternative technique to anchor RAFT agents via usage of macroRAFT agents has been introduced, which may combine the strengths of the Zand R-group approach. ${ }^{[140]}$ In this approach, a monomer with trimethoxysilyl side groups was polymerized first via RAFT polymerization to obtain macromonomers with RAFT end-groups. These macromonomers were subsequently coupled to silica particles through reaction with the trimethoxysilyl side groups, therefore neither the Z-group nor the R-group was directly fixed to the surface.

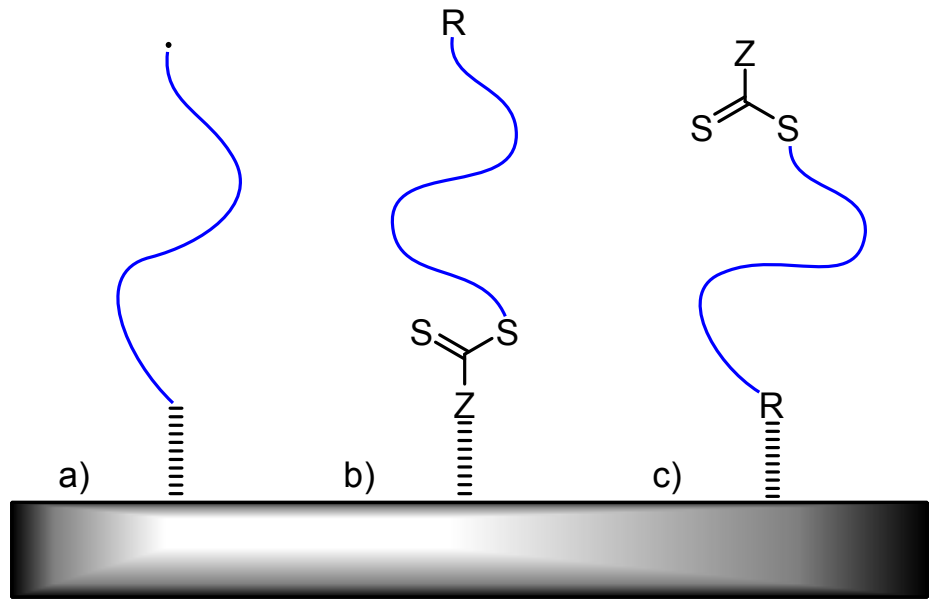

Figure 3-3. Different "grafting-from" methods for the synthesis of surface-anchored polymer: a) immobilized radical initiator, b) Z-group supported RAFT agent, c) R-group supported RAFT agent. 


\subsection{Mechanical Testing of Polymers}

Mechanical testing of polymers is essential for designing high-performance plastic materials for selected applications. The following subchapters outline two commonly applied techniques that were used in this work, which are tensile testing and DMA.

\subsubsection{Tensile Testing}

The widespread tensile test is probably one of the most convenient techniques to analyze the mechanical strength of a polymer. ${ }^{[141]}$ It may deliver several relevant properties concerning the quality of a polymer as a material such as rigidity, brittleness, ductility, and elastic behavior. ${ }^{[41]}$

In a tensile test a rectangular or dumbbell-shaped specimen is drawn with constant speed. The sample hence experiences an elongation $\varepsilon$, which is the deformation $\Delta L$ relative to the original length $L_{0}$ of the specimen:

$$
\varepsilon=\frac{\Delta L}{L_{0}}
$$

Together with the strain the tensile stress $\sigma$ is recorded, which is the force $F$ acting on the cross section area $A_{0}$ of the sample:

$$
\sigma=\frac{F}{A_{0}}
$$

A plot of measured tensile stress against tensile strain yields the stress-strain curve, from which specific values of interest can be extracted and which collectively describes the mechanical response of a polymer specimen towards tension (Figure 3-4). The area under the stress-strain plot represents the total energy that is required for breaking the sample and is hence a measure for the toughness of the material, denoted $\tau$ in the thesis in hand:

$$
\tau=\int_{0}^{\varepsilon_{B}} \sigma d \varepsilon,
$$

where $\varepsilon_{\mathrm{B}}$ is the elongation at break.

All stress-strain curves exhibit an elastic regime at very small deformations, in which the tensile stress is directly proportional to the lengthwise strain:

$$
\sigma=E \cdot \varepsilon .
$$


The proportionality constant $E$ is the tensile modulus, also often termed Young's modulus. In this part of the tensile test the polymer is said to obey Hooke's law and the tensile modulus can be determined via regression of the data points at small strain (usually between $0.05 \%$ and $0.25 \%$ strain). Beyond small deformations the appearance of the stress-strain curve strongly depends on the type of polymer and the testing conditions (temperature, elongation speed etc.). ${ }^{[41]}$

Many thermoplastic polymers, which are elongated below their glass transition temperature, exhibit a break point, defined by $\varepsilon_{\mathrm{B}}$ and the stress at break, $\sigma_{\mathrm{B}}$, that occurs in the linear regime of the stress-strain curve. These materials are classified as brittle and are characterized by a high tensile modulus and a low elongation at break. ${ }^{[141]}$

Other thermoplastics may display a ductile behaviour and undergo high deformations before the break point, such as the polymer sample in Figure 3-4. In this case the elongation behavior can further be characterized by a yield point with the coordinates yield stress, $\sigma_{\mathrm{Y}}$, and strain at yield, $\varepsilon_{\mathrm{Y}}$. This point is defined as the first point where the slope of the stress-strain curve becomes zero, ${ }^{[142]}$ that is an increase of strain is observed without an increase in stress. ${ }^{[41]}$ Beyond this point, the specimen undergoes permanent deformations which result in a reorientation of its chains on a large scale. The yield point hence reflects the limit of irreversible deformations. ${ }^{[141]}$

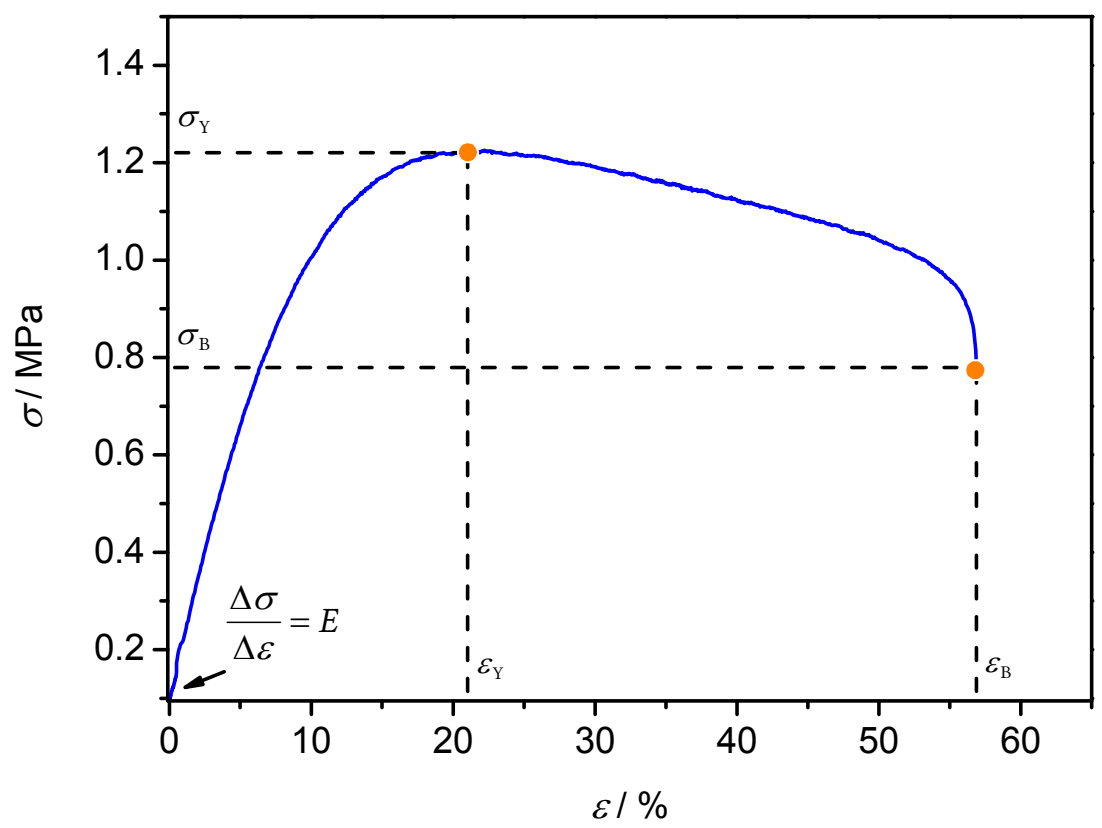

Figure 3-4. Stress-strain curve of poly(styrene ${ }_{396}-$ block- $\left.n \mathrm{BA}_{232}\right)$ with indicated yield and break points and the corresponding stresses and strains. Elongation speed: $25 \mathrm{~mm} \cdot \mathrm{min}^{-1}$ for strains before $0.25 \%$ and $100 \mathrm{~mm} \cdot \mathrm{min}^{-1}$ afterwards. 


\subsubsection{Dynamic Mechanical Analysis}

DMA provides access to the viscoelastic parameters of a sample and is therefore applied to materials which exhibit both elastic and viscous behavior. ${ }^{[44,141,143,144]}$

For purely elastic materials the deformation caused by an external force is fully reversible and independent of time. In this case the deformed specimen recovers its initial shape immediately upon removal of the stress and the energy originally applied to the sample is fully recovered. Such materials are said to behave Hookean and hence the strain is directly proportional to the stress similar to the linear part of the stress-strain curve described in the previous subchapter. ${ }^{[141]}$

Completely viscous materials do not deform but flow under an external stress:

$$
\sigma=\eta \cdot \dot{\varepsilon}
$$

with $\eta$ being the viscosity and $\dot{\varepsilon}$ the rate of strain. Therefore the response of such an ideal liquid is by definition time-dependent. ${ }^{[141]}$ Consequently the applied energy is completely transformed into heat via friction, which makes the recovery of the original structure impossible. ${ }^{[44]}$

Polymers represent a typical class of viscoelastic materials as they feature elastic as well as viscous characteristics, which is why DMA displays a very well suited mechanical characterization method for macromolecular systems. In DMA an oscillating stress (or strain) with frequency $\omega$ is applied to the sample and its resulting strain (or stress) is measured as a function of temperature or applied frequency. In this work, measurements were performed with application of stresses and thus the theoretical description will concentrate on this approach, although the difference is merely conceptual. ${ }^{[143]}$ The stress can mathematically be described as follows:

$$
\sigma^{*}(t)=\sigma_{0} \cdot \exp [i(\omega t+\delta)],
$$

where $\sigma(t)$ is the stress at a given time $t, \sigma_{0}$ denotes the maximum amplitude of the stress, and $\delta$ is the phase angle between stress and strain, also called loss angle. The strain can be similarly defined:

$$
\varepsilon^{*}(t)=\varepsilon_{0} \cdot \exp (i \omega t),
$$

with $\varepsilon(t)$ constituting the oscillating strain and $\varepsilon_{0}$ the maximum amplitude of strain. The resulting strain of elastic bodies will be completely in phase with the stress (instant recovery) and $\delta$ will be $0^{\circ}$, whereas for viscous materials the two signals will be out of phase and $\delta$ will then be $90^{\circ}$. Viscoelastic materials will 
exhibit a behavior in between these two extremes $\left(0^{\circ}<\delta<90^{\circ}\right)$ as depicted in Figure 3-5.

From the ratio of oscillating stress to strain a complex modulus may be defined: $:^{[44]}$

$$
E^{*}=\frac{\sigma^{*}}{\varepsilon^{*}}=E^{\prime}(\omega)+i E^{\prime \prime}(\omega)
$$

where $E^{*}$ is the elastic modulus and $E^{\prime}$ and $E^{\prime \prime}$ are referred to as the storage and the loss modulus, respectively. The latter represents the amount of energy which is dissipated into heat per cycle within the sample, whereas the storage modulus displays the amount of energy that is stored by the sample during one cycle. The ratio of these two moduli gives the mechanical damping parameter, $\tan \delta$ :

$$
\tan \delta=\frac{E^{\prime \prime}(\omega)}{E^{\prime}(\omega)}
$$

This parameter directly yields the ratio of viscous to elastic behavior and mirrors the damping capacity of the sample. ${ }^{[141]}$ It is particularly useful for determining molecular relaxations, which usually occur as a maximum in $\tan \delta^{[44]}$ The measurement of $\tan \delta$ as a function of temperature yields the $\tan \delta$-curve.

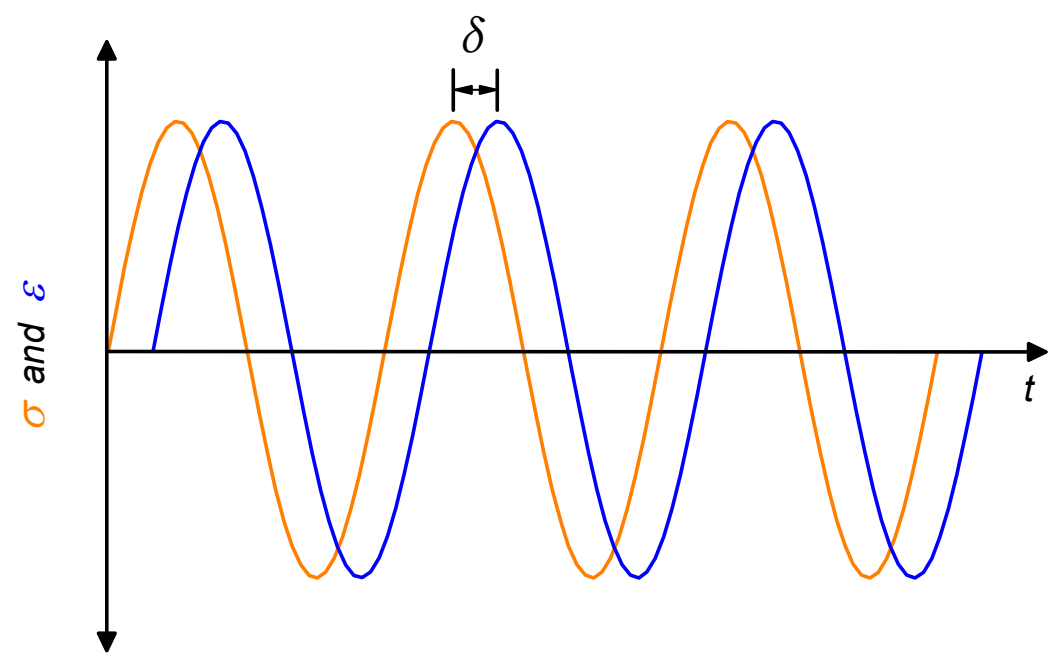

Figure 3-5. Schematic visualization of the applied stress $\sigma$ and the resulting strain $\varepsilon$ in DMA with the corresponding loss angle $\delta$. 


\subsection{Polymer Relaxations}

For amorphous polymers, various relaxations can be detected in $\tan \delta$-curves, which correspond to different molecular motions and are labelled with letters of the Greek alphabet in the order of decreasing temperature. ${ }^{[41,44]}$

At the primary $\alpha$-relaxation, commonly known as the glass transition at temperature $T_{\mathrm{g}}$, the polymer is transformed from a hard and glassy state into a soft and rubbery material upon increasing temperature. This glass relaxation is caused by an increase of the overall chain mobility and its temperature is dependent on characteristics such as molar mass, side groups, cross-links, degree of crystallinity, and adjuvants of the polymer. However, the influence of for example the molar mass as well as the composition of the polymer on the glass transition temperature is still under discussion. ${ }^{[145-147]}$

Below the glass transition temperature, a secondary $\beta$-relaxation is often detected, which may arise from side-group movements of the monomer units, though its mechanism is not fully clarified. ${ }^{[41]}$ This $\beta$-process is often of less intense and broader with respect to the temperature range compared with the glass transition.

a)

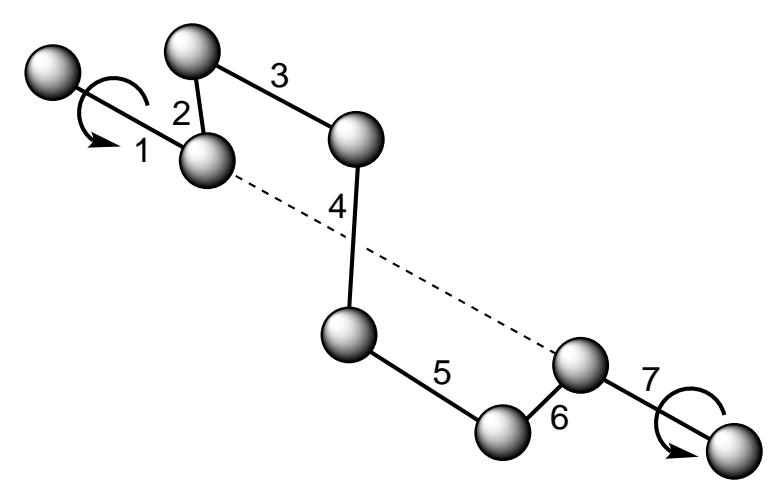

b)

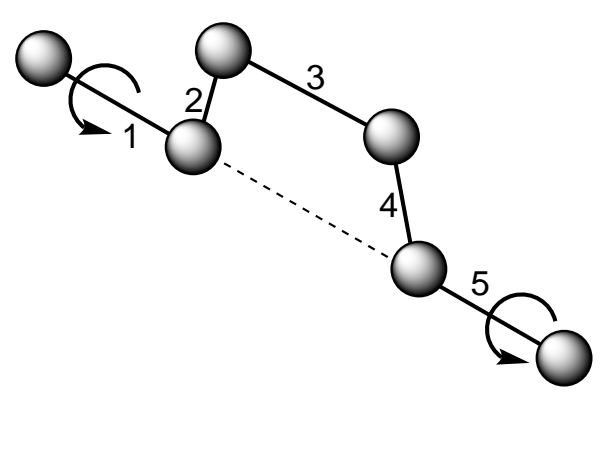

Figure 3-6. Crankshaft motions of polyethene segments according to Schatzki (a) and Boyer (b). ${ }^{[148]}$

At even lower temperatures, a $\gamma$-relaxation may be observed that is referred to crankshaft motions of main chain segments or side-group motions. ${ }^{[44,144,149]}$ According to proposals of Schatzki ${ }^{[150]}$ and Boyer, ${ }^{[111]}$ the crankshaft-like motions can be described by assuming polyethene chain segments of seven and five carbon-carbon bonds, respectively, which are arranged at constant angles (Figure 3-6). The actual motion is given by the rotation of the inner five and 
three bonds, respectively, around the two outmost bonds that are collinear. In acrylate and methacrylate polymers the $\gamma$-mechanism has been observed since the 1950's, in which case it is attributed to the independent motion of the alkyl side groups ${ }^{[149]}$ For these polymers, the temperature of this relaxation is largely depending on the nature of the corresponding alkyl group involved in the molecular motion. 


\section{PART 1:}

SURFACE-INITIATED POLYMERIZATIONS 



\section{Surface-Initiated PLP-SEC}

In this chapter the determination of $k_{\mathrm{p}}$ values for surface-initiated polymerizations of $n \mathrm{BA}$ and styrene via the state-of-the-art PLP-SEC method is presented along with reference experiments, in which DMPA and 2,2'-azo-bis(iso-butyronitrile) (AIBN) were applied as initiators in solution (Scheme 4-1). ${ }^{[152]}$

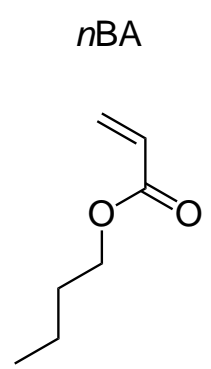

$$
\text { styrene }
$$

DMPA

AIBN

Scheme 4-1. Chemical structures of $n \mathrm{Ba}$, styrene, DMPA and AIBN.

Since its invention in the 1980 's ${ }^{[64]}$ the PLP-SEC technique has been applied to various polymerization systems including polymerizations of numerous monomers in bulk, ${ }^{[67,153-155]}$ copolymerizations, ${ }^{[156-158]}$ polymerizations at varying temperatures and pressures, ${ }^{[159]}$ polymerizations in different solvents ${ }^{[160]}$ such as water, ${ }^{[161-165]}$ supercritical $\mathrm{CO}_{2},{ }^{[166-169]}$ and ionic liquids, ${ }^{[170,171]}$ as well as in heterogeneous media. ${ }^{[172,173]}$ Surprisingly, a field which has not yet been in the focus of the PLP-SEC method is the one of surface-initiated polymerizations, which has generally received increasing attention in recent years due to the ability to produce tailored surfaces and materials with remarkable properties. $^{[30,31,174]}$

This work demonstrates the first assignment of $k_{\mathrm{p}}$ values for radical polymerizations from a solid support via the PLP-SEC setup. Earlier works by Rühe et al. ${ }^{[33]}$ have shown that the propagation mechanism of grafted radicals is 
similar to the chain growth mechanism in solution, whereas the termination reaction is more likely to feature discrepancies between free and fixed macroradicals. However, while their work covered the overall reaction rate for the graft polymerization, the thesis in hand is dedicated to the determination of individual $k_{\mathrm{p}}$ values for the surface-initiated radical polymerization of $n \mathrm{BA}$ and styrene. With this approach, even small differences in $k_{\mathrm{p}}$ are expected to be detectable. As the IUPAC benchmark $k_{\mathrm{p}}$ values for $n \mathrm{BA}$ and styrene differ largely in size, the use of these two monomers further allows for the comparison of diverse propagation rates that might have different impacts on radical chain growth from a solid support.

In the first part of this chapter the immobilized initiator and the proposed mechanism for surface-initiated PLP-SEC experiments are introduced. Afterwards the MMDs of the obtained polymers and the extracted $k_{\mathrm{p}}$ values are presented and deviations from IUPAC benchmark values are discussed.

\subsection{The Silica-Immobilized Initiator}

The experimental setup used for obtaining propagation rate coefficients of surface-initiated polymerizations is rather similar to conventional PLP-SEC setups. The main difference is eventually the use of an immobilized UV-initiator that is suspended in the monomer solution for subsequent PLP from the solid substrate.
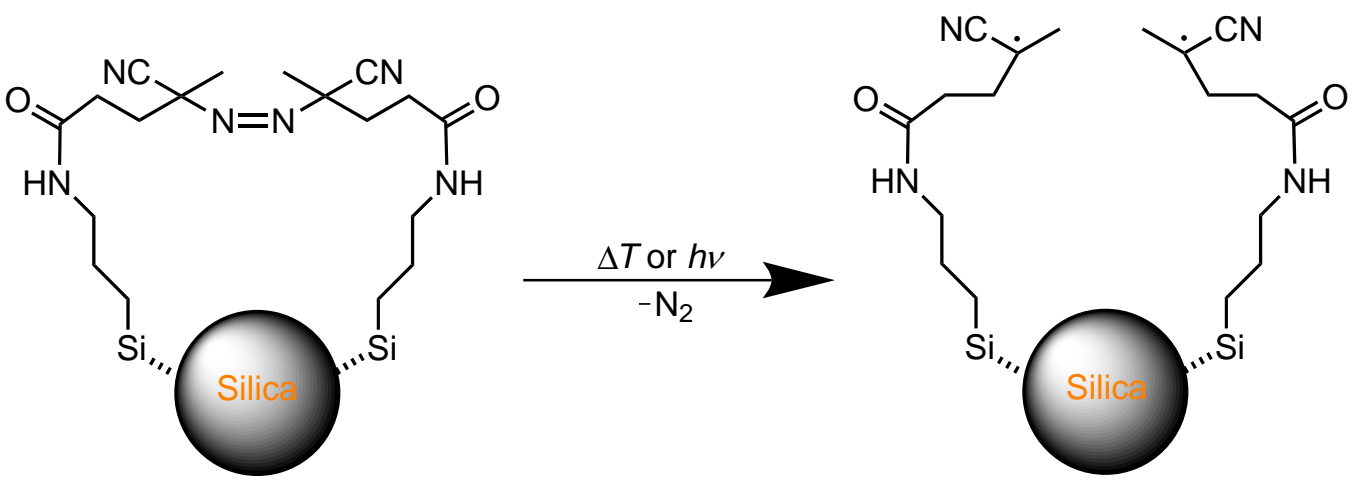

Figure 4-1. Silica-immobilized 4,4'-azobis(4-cyano- $N$-(3"-triethoxysilylpropyl)-valeric amide) (Si-ACTA).

The employed initiator 4,4'-azobis(4-cyano- $N$-(3"-triethoxysilylpropyl)valeric amide) (ACTA) ${ }^{[35]}$ (Figure 4-1) can be anchored to any surface containing hydroxyl groups, in which course nearly all of the initiator is bound to the 
surface given that free hydroxyl groups are still present. Therefore the grafting density of the initiator can be easily tuned in that case by simply using a defined amount of free initiator and silica particles. Since ACTA is linked to the surface via two anchor groups, radicals are exclusively produced at the surface and therefore no interference from reactions in the solution phase is present as long as transfer reactions have not triggered the polymerization there as well. This great enhancement of surface-initiated polymerizations via the use of bipedal initiators compared to single-attached initiators has just recently been introduced ${ }^{[35,117]}$ One possible deficiency, which is particularly crucial for PLPSEC experiments, is the fact that the production of radicals from photoexcitation of azo alkanes, as for example AIBN and the anchored ACTA, is noninstantaneous on the PLP timescale and can hence lead to a distortion of the polymer's $\mathrm{MMD},{ }^{[175]}$ especially for monomers with a high $k_{\mathrm{p}}$ value like $n \mathrm{BA}$. This issue will be highlighted later in this chapter.

For the PLP-SEC experiments in this work silica nanoparticles were used as the solid support because of their chemical resistance, mechanical stability, relatively low costs, and high specific surface area. Although substantial scattering and reflection of the UV light used for initiation (351 nm) probably occurs due to agglomeration of the suspended silica particles, the overall absorbance should be low enough to enable the laser pulses to penetrate the reaction mixture. ${ }^{[176]}$ Because of the significant differences in propagation and termination rates of the two monomers $n \mathrm{BA}$ and styrene, different laser pulse repetition rates were applied for PLP, namely $100 \mathrm{~Hz}$ for $n \mathrm{BA}$ and $5 \mathrm{~Hz}$ for styrene.

\subsection{Mechanism}

Like the experimental setup, the proposed mechanism for PLP-SEC from solid surfaces, as shown in Figure 4-2, is also strongly related to conventional PLPSEC measurements. Laser pulses are applied to trigger the decomposition of the grafted initiator ACTA, forming surface-tethered radicals, which can subsequently grow by adding monomer molecules that surround the silica particles. The next laser pulse creates a new population of anchored radicals, which should cause most of the growing chains to terminate and hence produce grafted polymer of well-defined chain lengths.

A fact that might hinder the formation of macromolecules with well-defined chain lengths-which is a prerequisite for obtaining $k_{\mathrm{p}}$ values-is the exclusive production of anchored radicals. As mentioned above, the benefit of this situation is that polymerization should only take place on the surface and hence no possibly disturbing termination between grafted and free chains can occur. However, this might simultaneously be a drawback as the forced termination 
events at each laser pulse also rely, to some extent, on the mobility of newly formed radicals. Since all radicals that emerge from $\mathrm{Si}-\mathrm{ACTA}$ are anchored and thus restricted in their movement, the rate of termination of growing radicals may be decreased. This, in turn, would result in a substantial fraction of growing radicals that propagate beyond the laser pulse, in which case the PLP structures in the MMDs of the polymers are distorted. However, in the event of MMDs with well-defined PLP structures, the mechanism should proceed as described above.

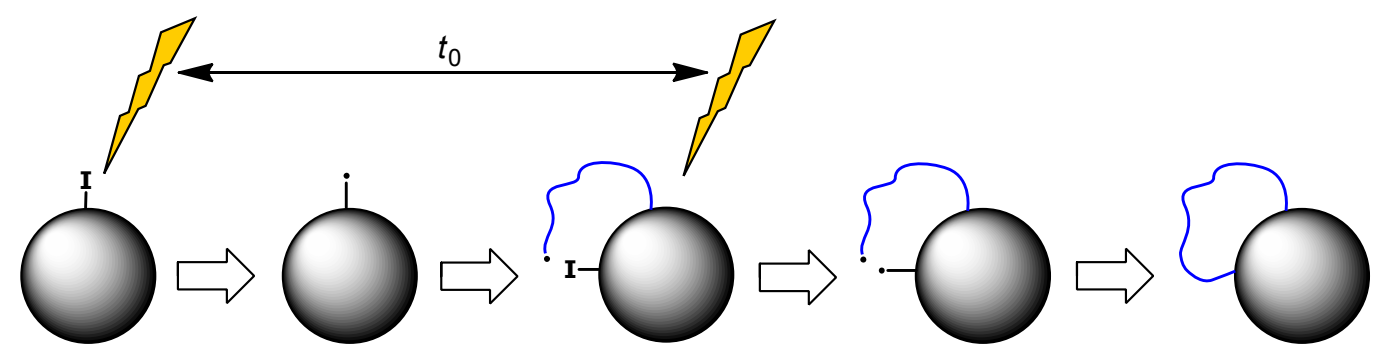

Figure 4-2. The proposed surface-initiated PLP-SEC process. The last two reaction steps are significantly shorter than the dark time period $t_{0}$.

\subsection{Results and Discussion}

At first, reference experiments of $n \mathrm{BA}$ in solution initiated by DMPA and AIBN, respectively, are presented. Afterwards, the results of the surface-initiated PLP of $n \mathrm{BA}$ and styrene are covered.

\subsubsection{Conventional PLP-SEC of $n \mathrm{BA}$}

As stated in chapter 4.1 azo initiators may hamper the success of PLP-SEC experiments due to their non-instantaneous decay on the PLP timescale. ${ }^{[175]}$ In order to check the suitability of ACTA for PLP-SEC, reference experiments were performed in which $n \mathrm{BA}$ was polymerized via PLP initiated by DMPA-a well-suited PLP initiator-and AIBN as a model initiator for the immobilized ACTA. Free ACTA was not directly used in solution as it yields polymer with highly reactive silyl end-groups, which are prone to undergo crosslinking reactions ${ }^{[177]}$ and thus distortion of the SEC signals would most probably occur. By comparing the MMDs of the poly $(n \mathrm{BA})$ produced by initiation of DMPA and AIBN, respectively, the suitability of ACTA for PLP-SEC can thus be indirectly assessed. 


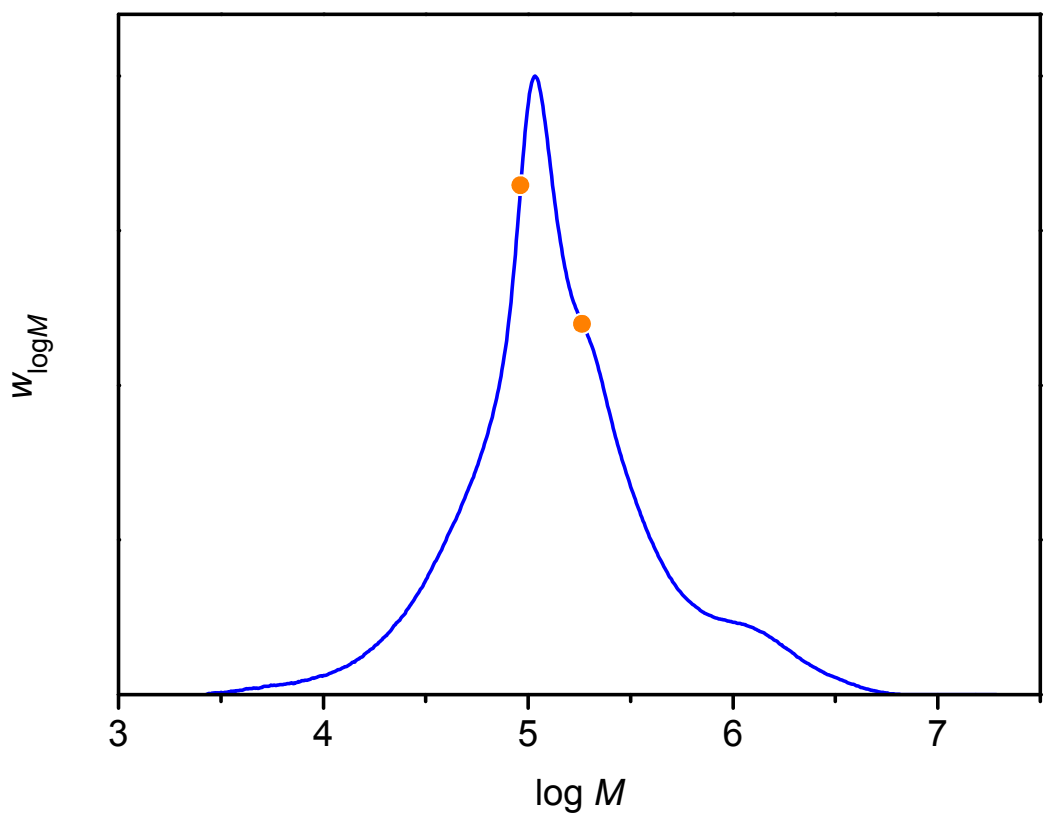

Figure 4-3. MMD (SEC trace) of poly $(n \mathrm{BA})$ with indicated points of inflection from PLP of $n \mathrm{BA}$ in bulk $\left(7.17 \mathrm{~mol} \cdot \mathrm{L}^{-1}\right)$ at $5{ }^{\circ} \mathrm{C}$ and $100 \mathrm{~Hz}$ initiated by DMPA $\left(1.98 \mathrm{mmol} \cdot \mathrm{L}^{-1}\right)$.

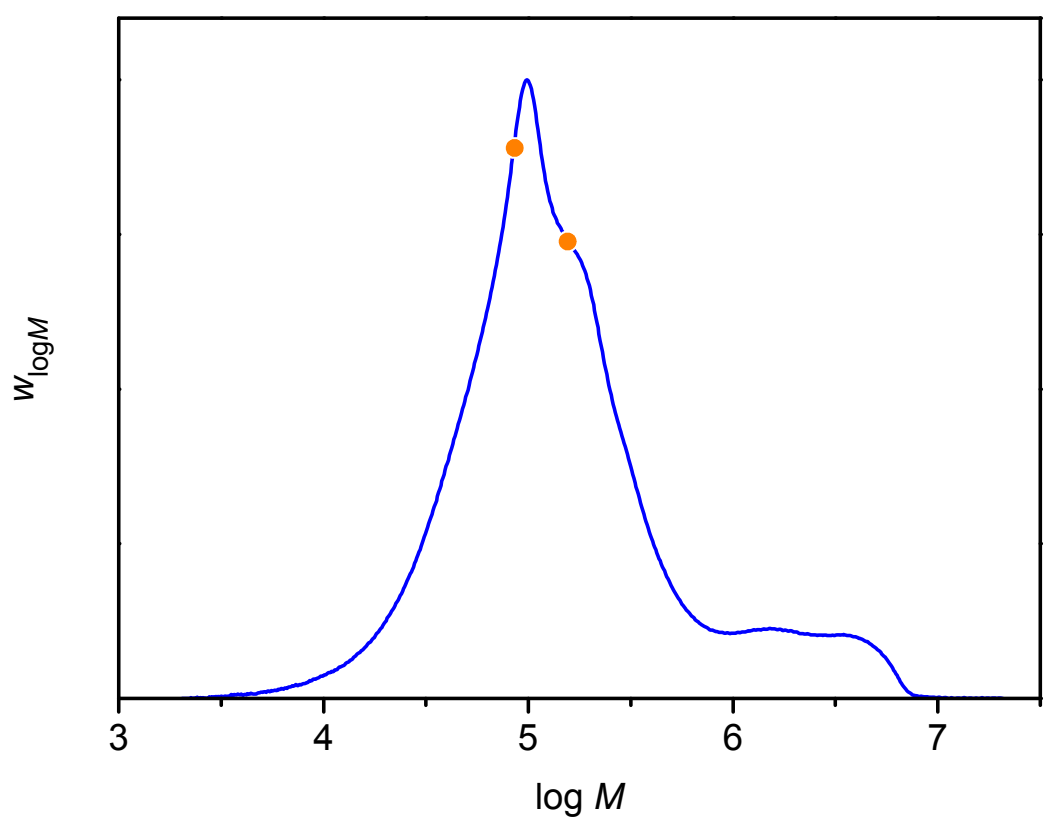

Figure 4-4. MMD (SEC trace) of poly $(n \mathrm{BA})$ with indicated points of inflection from PLP of $n \mathrm{BA}$ in bulk $\left(7.17 \mathrm{~mol} \cdot \mathrm{L}^{-1}\right)$ at $5{ }^{\circ} \mathrm{C}$ and $100 \mathrm{~Hz}$ initiated by $\operatorname{AIBN}\left(2.25 \mathrm{mmol} \cdot \mathrm{L}^{-1}\right)$. 
As expected, PLP-SEC of $n \mathrm{BA}$, initiated by DMPA at $5{ }^{\circ} \mathrm{C}$, yields poly $(n \mathrm{BA})$ with two very distinct inflection points that obey the consistency criterion (Figure 4-3). In comparison, initiation by AIBN produces poly $(n \mathrm{BA})$ with a slightly broader MMD displaying more high-molar-mass material (Figure 4-4). However, the MMD also features two well-located points of inflection. The extracted propagation rate coefficients are in good agreement with the IUPAC benchmark data (Table 4-1). Interestingly, the $k_{\mathrm{p}}$ values obtained from PLP via initiation with AIBN are even closer to the literature values than the ones derived from the PLP experiments initiated by DMPA. Hence AIBN appears to be well-suited for the determination of $k_{\mathrm{p}}$ values for $n \mathrm{BA}$ under the given reaction conditions. This confirms earlier investigations ${ }^{[175]}$ and indicates that the silica-immobilized ACTA may also be capable of producing polymers with MMDs that display PLP structures despite its azo-type nature.

\subsubsection{Surface-Initiated PLP-SEC of $n \mathrm{BA}$}

The PLP-SEC experiments of $n \mathrm{BA}$ initiated by Si-ACTA were carried out at temperatures of $25{ }^{\circ} \mathrm{C}$ (Figure 4-5) and $5{ }^{\circ} \mathrm{C}$ (Figure 4-6). In both cases, substantial amounts of poly $(n \mathrm{BA})$ in solution (dotted line) were present in spite of the low monomer conversion, which however coincides with earlier findings and is probably in all cases caused by transfer reactions from surface-tethered radicals to monomer molecules. ${ }^{[35,117]}$ In addition to that, the SEC signals of the poly $(n \mathrm{BA})$ in solution exhibit a low-molar-mass shoulder which seems to be more apparent at lower temperatures, but whose origin is unclear.

Compared with the grafted poly $(n \mathrm{BA})$, the main fraction of the $\operatorname{poly}(n \mathrm{BA})$ in solution is at higher molar masses at both temperatures and features no PLP structure, which is expected because of the absence of photoinitiator radicals in solution. The MMD of the immobilized poly $(n \mathrm{BA})$, polymerized at $25{ }^{\circ} \mathrm{C}$, also appears broad and indistinct with only one inflection point, which prevents the determination of the propagation rate coefficient.

However, acrylates in general are hard to study via PLP-SEC ${ }^{[178]}$ as side reactions such as intramolecular chain transfer ("backbiting") often destroy the PLP structure, especially at elevated temperatures, because of the existence of two propagating radical species, namely secondary propagating radicals (SPRs) and mid-chain radicals (MCRs). ${ }^{[155,178]}$ The MCRs that originate from the backbiting reactions exhibit a much slower propagation rate and lead to a broadening of the MMD, which can eventually result in the loss of the PLP structure. $n \mathrm{BA}$ has been intensively studied with regard to this difficulty and it was concluded that well-structured MMDs cannot be obtained at temperatures above $30{ }^{\circ} \mathrm{C}$ when using repetition rates of up to $100 \mathrm{~Hz} \cdot{ }^{[15]}$ For this reason, the failure to determine $k_{\mathrm{p}}$ values for the surface-initiated polymerization of $n \mathrm{BA}$ 
at $25{ }^{\circ} \mathrm{C}$ may be due to the high concentration of MCRs that disrupt the PLP process and the resulting MMD.

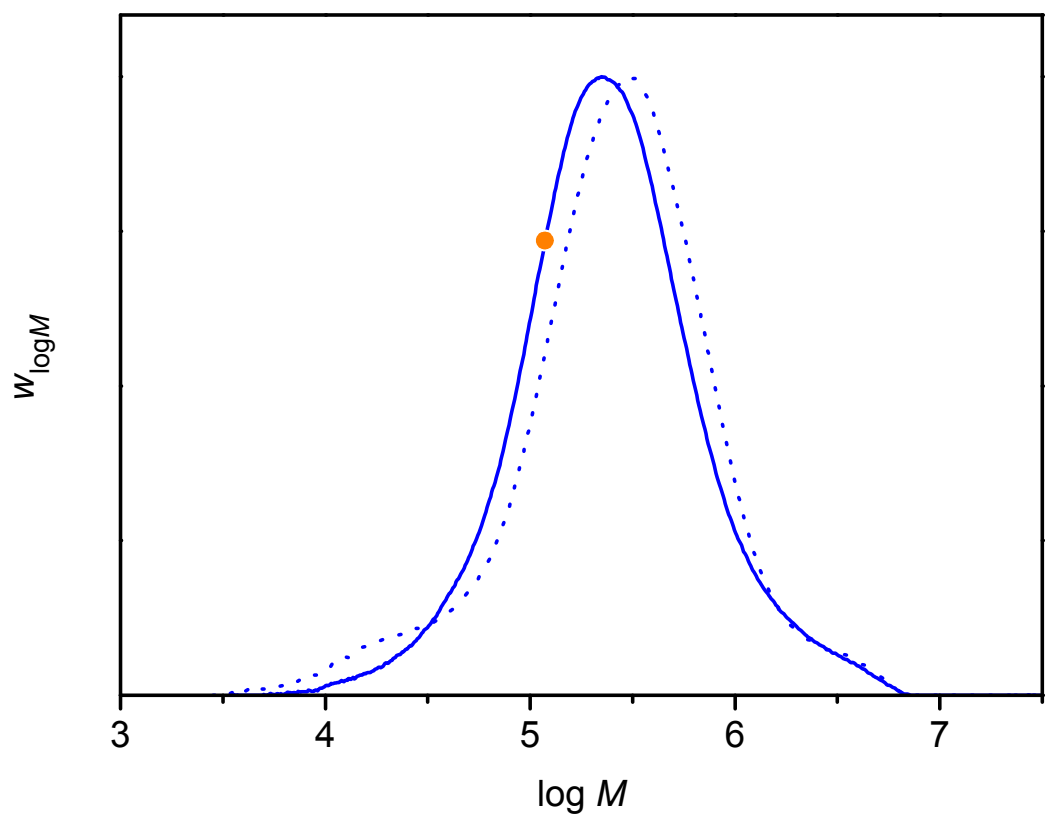

Figure 4-5. MMDs (SEC traces) of poly $(n \mathrm{BA})$ from PLP of $n \mathrm{BA}$ in bulk $\left(6.98 \mathrm{~mol} \cdot \mathrm{L}^{-1}\right)$ formed in solution (dotted line) and on the surface (full line with indicated point of inflection) at $25{ }^{\circ} \mathrm{C}$ and $100 \mathrm{~Hz}$ initiated by silica-immobilized ACTA $\left(20 \mathrm{mmol} \cdot \mathrm{L}^{-1}, 0.17\right.$ molecules $\left.\cdot \mathrm{nm}^{-2}\right)$.

In that case, a reduction of the reaction temperature from $25^{\circ} \mathrm{C}$ to $5{ }^{\circ} \mathrm{C}$ should enhance the characteristic PLP features of the MMD of the silica-anchored poly $(n \mathrm{BA})$ and enable the estimation of $k_{\mathrm{p}}$ from the SPR species only, as shown in Figure 4-6. In that case the MMD exhibits two inflection points with the second located at twice the molar mass of the first one. From the first inflection point the propagation rate coefficient can be estimated to be $k_{\mathrm{p}}=11695 \mathrm{~L} \cdot \mathrm{mol}^{-1} \cdot \mathrm{s}^{-1}$ (Table $4-1$ ), which is about $22 \%$ higher than the IUPAC benchmark value of $k_{\mathrm{p}}=9616 \mathrm{~L} \cdot \mathrm{mol}^{-1} \cdot \mathrm{s}^{-1} \cdot{ }^{[155]}$ Therefore, the amount of backbiting reactions and hence the concentration of anchored MCRs seems to be low enough at $5{ }^{\circ} \mathrm{C}$ to enable the determination of $k_{\mathrm{p}}$, which is in line with earlier findings. ${ }^{[155]}$ 


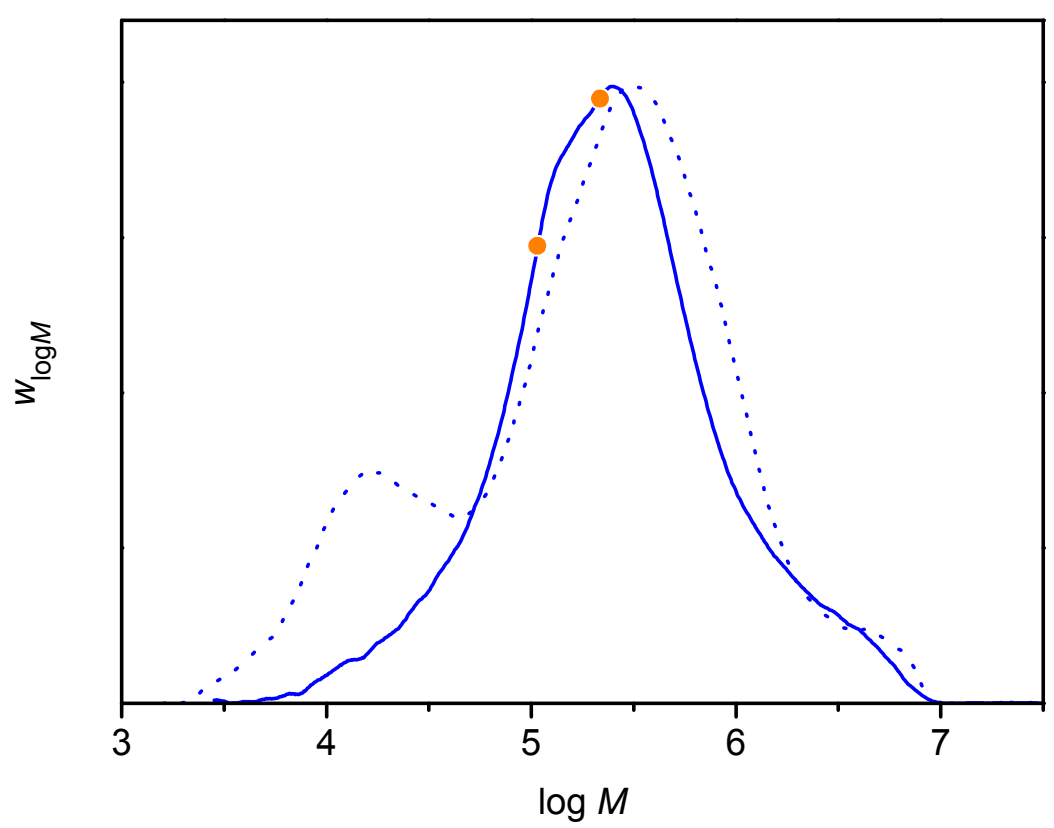

Figure 4-6. MMDs (SEC traces) of poly $(n \mathrm{BA})$ from PLP of $n \mathrm{BA}$ in bulk $\left(7.17 \mathrm{~mol} \cdot \mathrm{L}^{-1}\right)$ formed in solution (dotted line) and on the surface (full line with indicated points of inflection) at $5{ }^{\circ} \mathrm{C}$ and $100 \mathrm{~Hz}$ initiated by silica-immobilized ACTA $\left(20 \mathrm{mmol} \cdot \mathrm{L}^{-1}, 0.17\right.$ molecules $\left.\cdot \mathrm{nm}^{-2}\right)$.

\subsubsection{Surface-Initiated PLP-SEC of Styrene}

In comparison with $n \mathrm{BA}$, PLP of styrene, initiated by silica-tethered ACTA, produces polystyrene with MMDs that feature up to four visible inflection points (Figure 4-7). Additionally, no polymer in solution is formed in case of styrene, as transfer reactions are not pronounced in this system. The exclusive formation of radicals on the surface proves hence to be very advantageous. Furthermore the absence of significant amounts of side reactions in this system improves the quality of the determined MMDs also for temperatures close to room temperature (RT).

The extracted propagation rate coefficients from this and other experiments, listed in Table 4-1, deviate to higher values by approximately $23 \%$ compared with the IUPAC benchmark values of $k_{\mathrm{p}}=86 \quad \mathrm{~L} \cdot \mathrm{mol}^{-1} \cdot \mathrm{s}^{-1}$ and $k_{\mathrm{p}}=90 \mathrm{~L} \cdot \mathrm{mol}^{-1} \cdot \mathrm{s}^{-1}$ at $25^{\circ} \mathrm{C}$ and $26{ }^{\circ} \mathrm{C}$, respectively. ${ }^{[67]}$ 


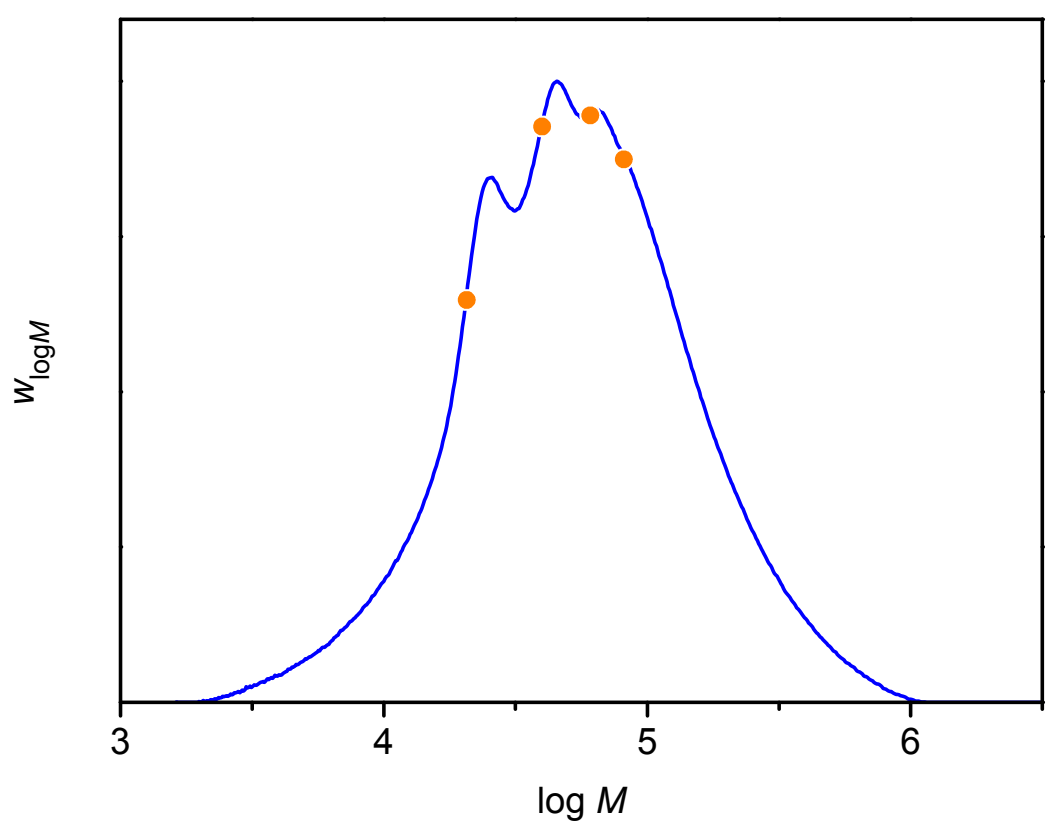

Figure 4-7. MMD (SEC trace) of polystyrene with indicated points of inflection from PLP of styrene in bulk $\left(8.65 \mathrm{~mol} \cdot \mathrm{L}^{-1}\right)$ formed on the surface at $26^{\circ} \mathrm{C}$ and $5 \mathrm{~Hz}$ initiated by silicaimmobilized ACTA $\left(8.8 \mathrm{mmol} \cdot \mathrm{L}^{-1}, 0.068\right.$ molecules $\left.\cdot \mathrm{nm}^{-2}\right)$.

Table 4-1. Values of propagation rate coefficients, $k_{\mathrm{p}}$, from the positions of the first points of inflection for $n \mathrm{BA}$ and styrene (Sty) at ambient pressure for polymerizations in bulk solution and from fumed silica.

\begin{tabular}{|c|c|c|c|c|c|c|}
\hline monomer & initiator & $\begin{array}{c}\mathrm{T} \\
{\left[{ }^{\circ} \mathrm{C}\right]}\end{array}$ & $\begin{array}{l}t_{0}^{-1} \\
{[\mathrm{~Hz}]}\end{array}$ & $\begin{array}{c}c_{\mathrm{I}} \\
{\left[\mathrm{mmol} \cdot \mathrm{L}^{-1}\right]}\end{array}$ & \multicolumn{2}{|c|}{$\begin{array}{l}k_{\mathrm{p}} \quad \text { IUPAC } k_{\mathrm{p}} \\
{\left[\mathrm{L} \cdot \mathrm{mol}^{-1} \cdot \mathrm{s}^{-1}\right]}\end{array}$} \\
\hline$n \mathrm{BA}$ & DMPA & 5 & 100 & 1.98 & 10213 & \\
\hline$n \mathrm{BA}$ & AIBN & 5 & 100 & 2.25 & 9280 & $9616^{[155]}$ \\
\hline$n \mathrm{BA}$ & AIBN & 5 & 100 & 2.25 & 9447 & \\
\hline$n \mathrm{BA}$ & Si-ACTA & 5 & 100 & 20.0 & 11695 & \\
\hline Sty & $\mathrm{Si}-\mathrm{ACTA}$ & 25 & 5 & 8.80 & 95 & $86^{[67]}$ \\
\hline Sty & $\mathrm{Si}-\mathrm{ACTA}$ & 25 & 5 & 8.80 & 109 & 80 \\
\hline Sty & $\mathrm{Si}-\mathrm{ACTA}$ & 26 & 5 & 8.80 & 114 & \\
\hline Sty & $\mathrm{Si}-\mathrm{ACTA}$ & 26 & 5 & 8.80 & 115 & $90^{[67]}$ \\
\hline
\end{tabular}




\subsection{Conclusion}

First of all, the application of an initiator that is covalently bound to a solid substrate in PLP-SEC enables the determination of propagation rate coefficients for surface-initiated polymerizations. This result is already remarkable because of the above-mentioned possible restrictions which could arise from the exclusive formation of radicals on the surface. Apparently, the induced termination events that occur at each laser pulse seem to be sufficiently fast for creating polymer with defined chain lengths, which is clearly visible in the obtained MMDs. In case of $n \mathrm{BA}$, surface-initiated PLP-SEC at $25{ }^{\circ} \mathrm{C}$ did not produce MMDs with multiple points of inflection due to the higher concentration of MCRs at this temperature. However, by reducing the temperature from $25{ }^{\circ} \mathrm{C}$ to $5{ }^{\circ} \mathrm{C}$, a well-structured MMD was obtained that enabled the determination of $k_{\mathrm{p}}$.

One effect which may enable the feasibility of the presented approach of PLP-SEC from surfaces is the relapsing of growing tethered macroradicals towards the surface. Since the grafting density of the initiator is low, the anchored chains probably do not form a brush-like but rather a coil-like orientation. ${ }^{[179,180]}$ Hence their active center is closer to the initiator-derived radicals and the termination reaction may then be sufficiently fast. Another aspect is the possibility of termination between two radicals that are located on different silica particles. In this case the grafted macroradicals do not solely rely on initiator molecules that are bound to the same particle but are able to terminate with any nearby initiator-derived radical.

The obtained $k_{\mathrm{p}}$ values for $n \mathrm{BA}$ and styrene determined via this approach deviate to higher values compared with the IUPAC benchmark values. Surprisingly, the determined differences seem to show the same tendency for both monomers, although their $k_{\mathrm{p}}$ values and chain lengths in the experiment differ by orders of magnitude. Therefore, the effect appears to be general for chains propagating from a solid surface. Two possible effects that may cause these deviations are discussed in the following.

Although the usage of an anchored initiator yields polymers that show MMDs with typical PLP structures and termination thus seems to occur with sufficient pace, the above-mentioned restriction in the mobility of immobilized chains could artificially extend the chain-growth period that should be set by the dark time in between two laser pulses. This may be caused by a competition between the propagation of fixed radicals and the chain-end encounter process that precedes the termination reaction. As a result the chain lengths are higher, as the radicals have more time to grow, and apparently higher $k_{\mathrm{p}}$ values are obtained. However, this possible circumstance does not seem to be very pronounced, since this would otherwise prevent the determination of $k_{\mathrm{p}}$ values which obey the consistency criterion of multiple inflection points in the MMD. 
Additionally, the influence of such an effect would be very different for $n \mathrm{BA}$ and styrene as the latter propagates much more slowly, hence the obtained $k_{\mathrm{p}}$ values for styrene should then be closer to the IUPAC values, which is not the case.

Another reason may be the decreased shielding of the active propagating centre by the anchored macroradical chain. Such a drop in shielding could be caused by the hindrance in motion due to the surface-anchorage. Although the chains' conformation is probably rather coil-like as described above, the attached chain-end cannot move close to the active radical site and thus probably not shield it as much as macroradicals in solution. This explanation may be related to the chain length dependence of $k_{\mathrm{p}}$ observed by Olaj et al., ${ }^{[181-183]}$ where such an effect is mentioned as a possible reason for higher propagation rate coefficients determined at higher laser frequencies and hence for shorter chains. Such oligomeric chains may also not be able to effectively shield the propagating site similar to grafted chains. Indeed the obtained $k_{\mathrm{p}}$ values at high laser frequencies and the presented $k_{\mathrm{p}}$ values for surfaceinitiated polymerizations lie in the same range, which supports this hypothesis. 



\section{Surface-Initiated Polymerization of Styrene}

This chapter is dedicated to the thermally surface-initiated radical polymerization of styrene in bulk. In all experiments polystyrene was grafted from silica nanoparticles via the employment of immobilized ACTA (Figure 4-1 on page 28). In contrast to chapter 4, where the formation of radicals was achieved with laser pulses (photoinitiation), the polymerizations presented here are triggered by thermal decomposition of the anchored initiator at $60{ }^{\circ} \mathrm{C}$.

The application of anchored initiators constitutes a popular grafting-from method for the production of polymer films that are covalently bound to a solid substrate. ${ }^{[26,28,33-35,115-118]}$ The formation of anchored polymer chains is in most cases accompanied by the generation of free polymer, which is inevitable when single-anchored initiators are used..$^{[3,34,115,118]}$ However, also when doubleanchored initiators are used for polymerization, the production of free polymer is hard to circumvent. ${ }^{[35,117]}$ In that case, the free polymer chains arise from transfer reactions of grafted radicals to monomer species such as solvent or monomer. By adding a chain transfer agent (e.g. RAFT agent) to the interstitial solution phase, it is also possible to induce a controlled radical polymerization both on the surface and in solution. ${ }^{[26]}$

The interplay of the polymerization on the surface and in solution is in many cases quite complex and the two types of polymers sometimes exhibit significant differences with respect to basic characteristics as for example the chain length, ${ }^{[33,35,15]}$ even though in many cases the free polymer's molar mass is claimed to reflect the molar mass of the anchored polymer without determination of the latter. ${ }^{[28,121]}$ Therefore, a thorough examination of the surface-initiated polymerization of styrene, which displays a well-known model monomer, via both the conventional and controlled radical approach, appears to be worthwhile in order to obtain a better comprehension of polymerizations from solid supports. Additionally, investigations into the propagation kinetics of styrene from fumed silica via PLP-SEC, presented in subchapter 4.3.3, have 
displayed significant differences compared with the propagation behavior in solution, which makes the system even more interesting.

The first part of this chapter highlights the conventional radical surfaceinitiated polymerization of styrene, whereas the second part outlines the consequences that arise from addition of RAFT agent to the polymerization. Both approaches are analyzed with respect to evolution of molar mass via SEC, with respect to polymer loading on the solid substrate via TGA, as well as with respect to surface topography via SEM. After SEC analysis and removal of the polymer produced in solution, the silica-polymer hybrids were analyzed by TGA and SEM and the grafted polymer was cleaved for subsequent SEC analysis. The surface-initiated RAFT polymerization is also investigated theoretically via a kinetic model developed in the program package PREDICI ${ }^{\circledR}$ with special emphasis on differences between the MMDs of the polymer formed in solution and on the surface.

\subsection{Surface-Initiated Conventional Radical Polymerization}

This very simple approach is carried out by suspending the silica modified with ACTA in styrene and exposing this suspension to elevated temperatures $\left(60^{\circ} \mathrm{C}\right)$ under vigorous stirring. The latter is especially important as the silica should be well-distributed in the reaction mixture, which is already quite viscous even before polymerization, to create the best possible homogeneity of the suspension.

\subsubsection{Evolution of Molar Mass}

The $M_{\mathrm{n}}$ and PDI values of the polystyrene produced at different total monomer conversions $X_{\mathrm{M}}$ are depicted in Figure 5-1 and listed in Table 5-1. Besides the grafted chains there is apparently a concomitant formation of polymer in solution, most probably caused by transfer reactions of anchored radicals, ${ }^{[35,117]}$ with a molar mass ranging from about $300000 \mathrm{~g} \cdot \mathrm{mol}^{-1}$ to $340000 \mathrm{~g} \cdot \mathrm{mol}^{-1}$. The silica-anchored polystyrene has lower molar masses ranging from $165000 \mathrm{~g} \cdot \mathrm{mol}^{-1}$ to $210000 \mathrm{~g} \cdot \mathrm{mol}^{-1}$, which is rather surprising as the fixed polymer usually exhibits higher masses compared with the free polymer. ${ }^{[33,35,115]}$ This circumstance may be attributed to a very low radical concentration in solution since transfer reactions of surface-bound radicals to molecules in solution are probably not very pronounced in the case of styrene. As a result, the rate of termination could be dramatically decreased in solution and higher molar masses are hence obtained. Moreover, the PDI values differ for the two 
polymers. The polymer in solution exhibits typical PDI values slightly below 2.0 , whereas the fixed chains display higher PDI values ranging from 2.30 to 2.75. In both cases, no dependency of molar mass on monomer conversion is found as expected of a conventional radical polymerization.

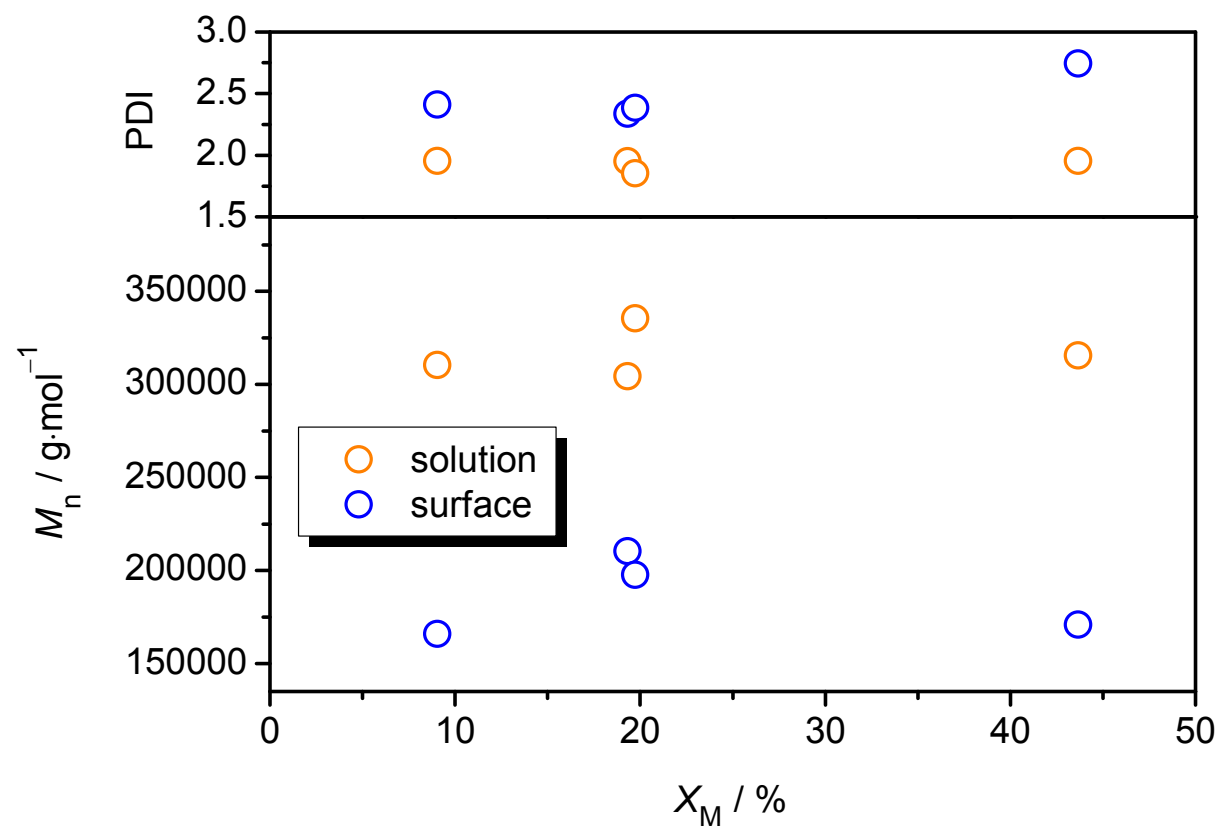

Figure 5-1. Evolution of number-average molar mass $M_{\mathrm{n}}$ and PDI with total monomer conversion $X_{\mathrm{M}}$ for the surface-initiated polymerization of styrene $\left(8.36 \mathrm{mmol} \cdot \mathrm{L}^{-1}\right)$ initiated by silica-anchored ACTA $\left(5.6 \mathrm{mmol} \cdot \mathrm{L}^{-1}, 0.069\right.$ molecules $\left.\cdot \mathrm{nm}^{-2}\right)$ at $60{ }^{\circ} \mathrm{C}$.

Table 5-1. Total monomer conversions $X_{\mathrm{M}}$, number-average molar masses $M_{\mathrm{n}}$, and PDI values of free and anchored polystyrene and calculated fractions of anchored polystyrene $f_{\text {polystyrene }}^{\text {sufer }}$ for the four silica-polymer composites obtained from surface-initiated polymerization of styrene $\left(8.36 \mathrm{mmol} \cdot \mathrm{L}^{-1}\right)$ initiated by anchored ACTA $\left(5.6 \mathrm{mmol} \cdot \mathrm{L}^{-1}, 0.069\right.$ molecules $\left.\cdot \mathrm{nm}^{-2}\right)$ at $60^{\circ} \mathrm{C}$.

\begin{tabular}{l|r|r|r|r|r|r}
\hline sample & $\begin{array}{c}X_{\mathrm{M}} \\
{[\%]}\end{array}$ & $\begin{array}{c}M_{\mathrm{n}}^{\text {solution }} \\
{\left[\mathrm{g} \cdot \mathrm{mol}^{-1}\right]}\end{array}$ & $\begin{array}{c}M_{\mathrm{n}}^{\text {surface }} \\
{\left[\mathrm{g} \cdot \mathrm{mol}^{-1}\right]}\end{array}$ & $\mathrm{PDI}^{\text {solution }}$ & $\mathrm{PDI}^{\text {surface }}$ & $f_{\text {polystyrene }}^{\text {surface }}$ \\
\hline $\mathbf{5 - 1}$ & 9.0 & 310360 & 165810 & 1.95 & 2.41 & 0.22 \\
$\mathbf{5 - 2}$ & 19.2 & 304350 & 210350 & 1.95 & 2.34 & 0.30 \\
$\mathbf{5 - 3}$ & 19.7 & 335530 & 197640 & 1.85 & 2.38 & 0.31 \\
$\mathbf{5 - 4}$ & 43.7 & 315570 & 170850 & 1.95 & 2.74 & 0.48 \\
\hline
\end{tabular}




\subsubsection{Polymer Loading}

The loading of grafted polystyrene on the silica particles can be determined via TGA after removal of the polymer formed in solution. The weight loss of the silica-polymer composites upon heating to $600{ }^{\circ} \mathrm{C}$ due to thermal decomposition of the organic polymer layer is proportional to the amount of grafted polystyrene. The TGA curves of the four hybrid materials, shown in Figure 5-2, illustrate the increase in relative weight loss of the polystyrene-functionalized silica particles towards higher total monomer conversion. The fraction of anchored polystyrene in the composites, $f_{\text {polystyrene }}^{\text {suface }}$ can be estimated by using the weight loss between $300{ }^{\circ} \mathrm{C}$ and $600{ }^{\circ} \mathrm{C}$ as a measure for $f_{\text {polystyrene }}^{\text {surface }}$. Although this approach neglects other sources of weight loss in this temperature range, the error of the obtained values should be small, as the weight loss is nearly completely caused by degradation of the polymer film.

The resulting polymer contents are listed in Table 5-1 together with the corresponding total monomer conversions. The fraction of silica-immobilized polymer increases steadily towards higher $X_{M}$ values and reaches a maximum of $44 \%$ for sample 5-4 at $43.7 \%$ total monomer conversion.

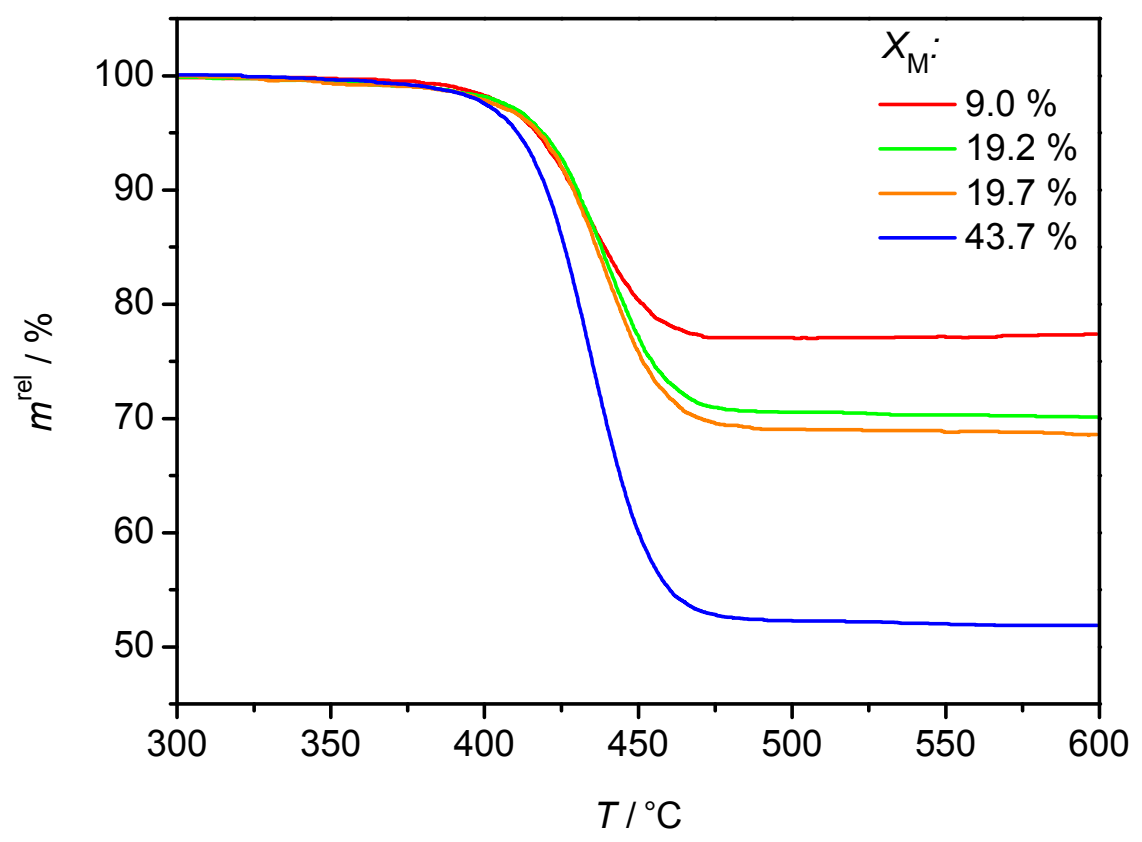

Figure 5-2. TGA curves from $300{ }^{\circ} \mathrm{C}$ to $600{ }^{\circ} \mathrm{C}$ of silica-polystyrene hybrids with different monomer conversions obtained from surface-initiated polymerization of styrene $\left(8.36 \mathrm{mmol} \cdot \mathrm{L}^{-1}\right)$ initiated by silica-anchored ACTA $\left(5.58 \mathrm{mmol} \cdot \mathrm{L}^{-1}, 0.069\right.$ molecules $\left.\cdot \mathrm{nm}^{-2}\right)$ at $60{ }^{\circ} \mathrm{C}$. Polymer fractions are listed in Table 5-1. 


\subsubsection{Surface Topography}

The surface topography of the silica-polystyrene hybrid materials was probed via SEM. Earlier investigations had shown that distinct differences were visible depending on the content of polymer in the composites and the molar mass of the grafted chains. ${ }^{[35,39]}$

The surface of the silica particles modified with ACTA (Figure 5-3) acts as reference for determining changes in the surface structure that are caused by the polymer layer. As expected, the surface of pure Si-ACTA appears rather porous and disordered which coincides with previous experimental findings. ${ }^{[35]}$

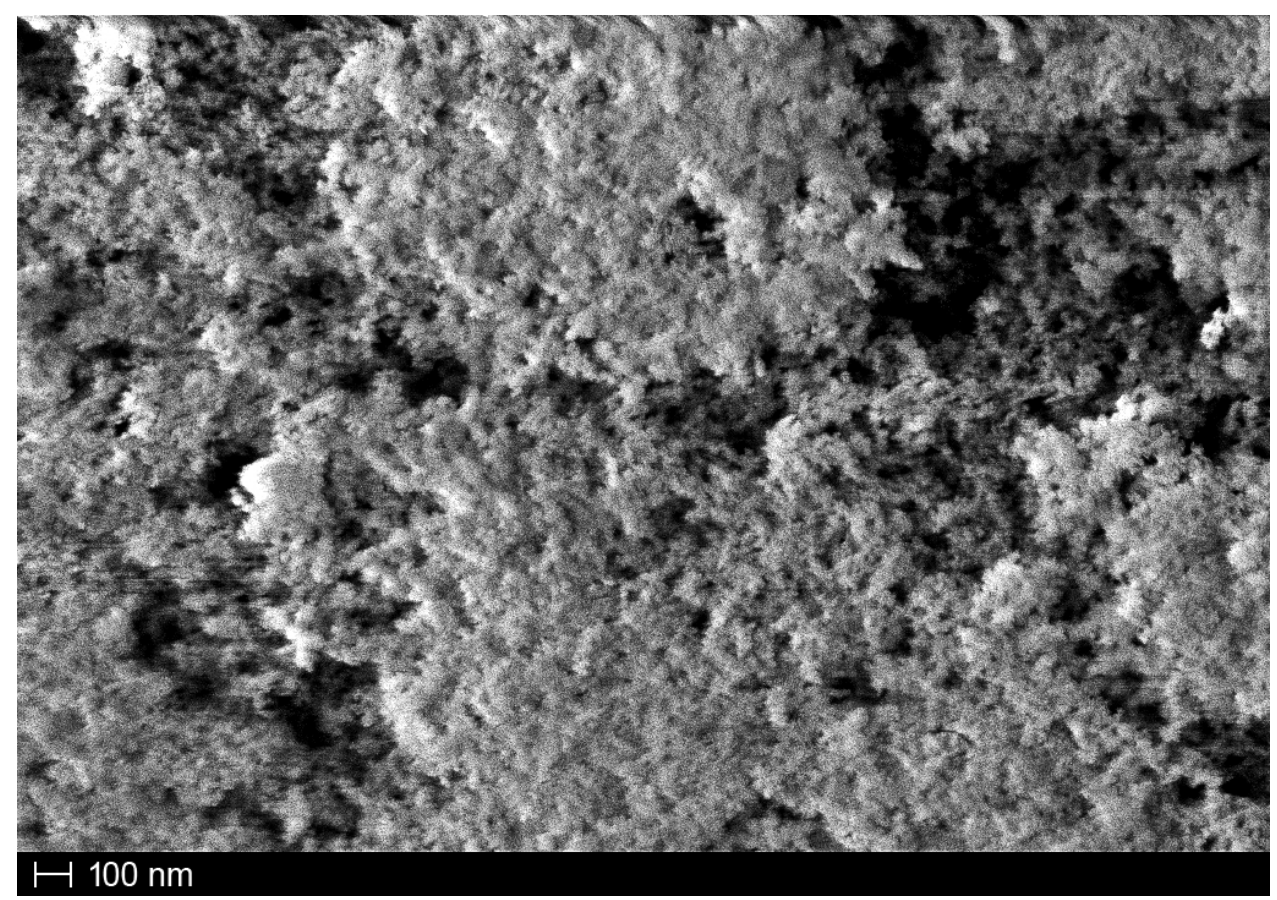

Figure 5-3. SE micrograph of $\mathrm{Si}-\mathrm{ACTA}$.

In contrast to that, the obtained SEM images of the polymer-functionalized silica samples (Figure 5-4 to Figure 5-7) reveal a more structured surface, which appears coarse-grained with decreased grain size at higher polymer loading (Figure 5-7). The polystyrene film may thus induce a separation of silica particles which is particularly effective at higher polymer contents, resulting in a more ordered composite surface. This effect is also in alignment with previously investigated poly(MA) films on the surface of fumed silica. ${ }^{[35]}$

The silica-polystyrene hybrid 5-4 obtained after $43.7 \%$ conversion with a polymer content of $44 \%$ exhibits several eye-catching areas that are most 
probably linked to the grafted polystyrene chains. Figure 5-8 shows a SE micrograph taken at lower magnification (10000-fold), in which some cracks in the material surface seem to be agglutinated by an additional substance. Two of these regimes are clearly visible at higher magnification (20000-fold) in Figure 5-9 and Figure 5-10. The surface cracks appear to be glued by filaments that very probably arise from the polystyrene layer. Although single macromolecules are not visible in SEM images, these filaments of approximately $1 \mu \mathrm{m}$ in length could have been formed by an ensemble of anchored polymer chains that cover the surface of the silica particles and are more clearly visible at the site of such cracks in the composite material.

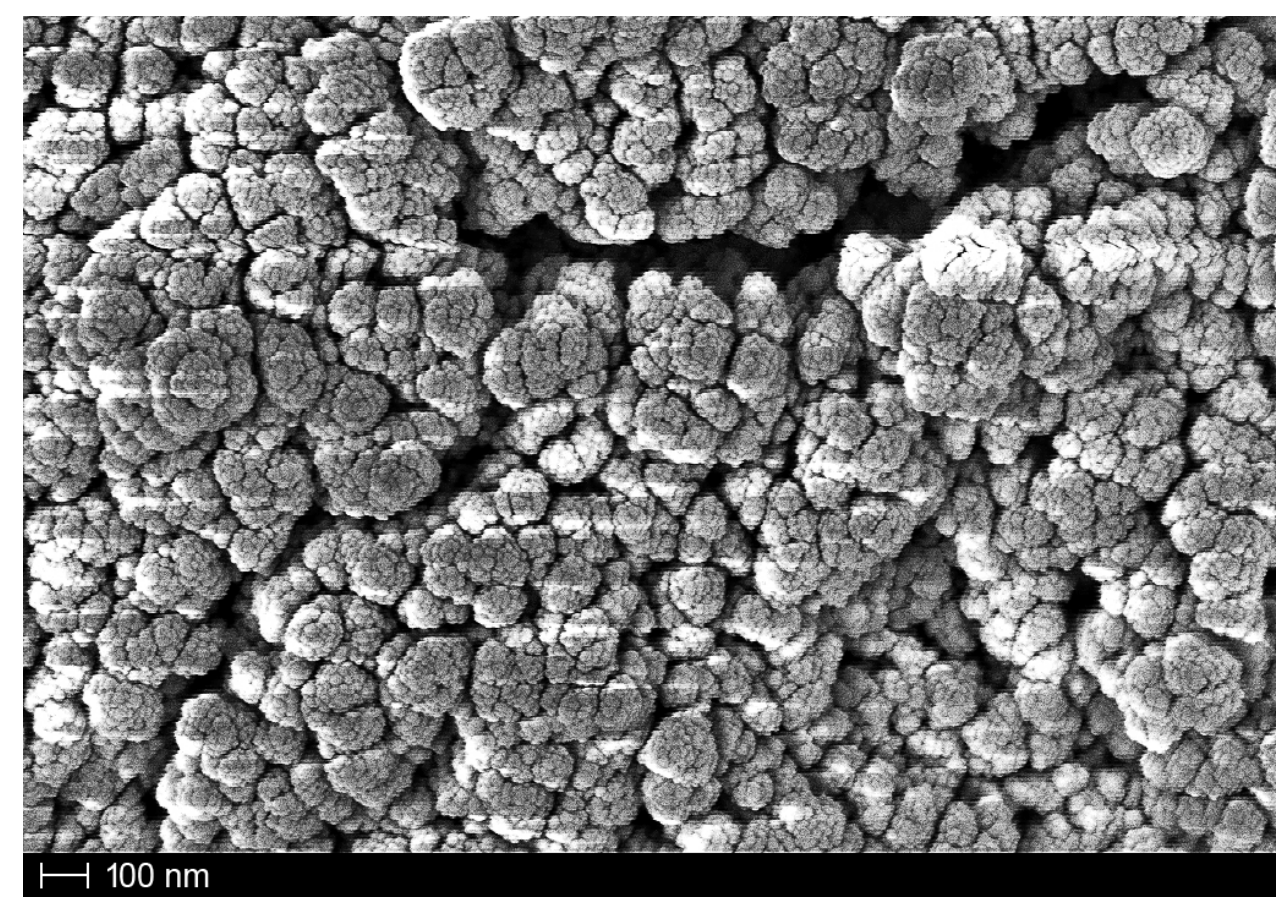

Figure 5-4. SE micrograph of silica-polystyrene hybrid 5-1. 


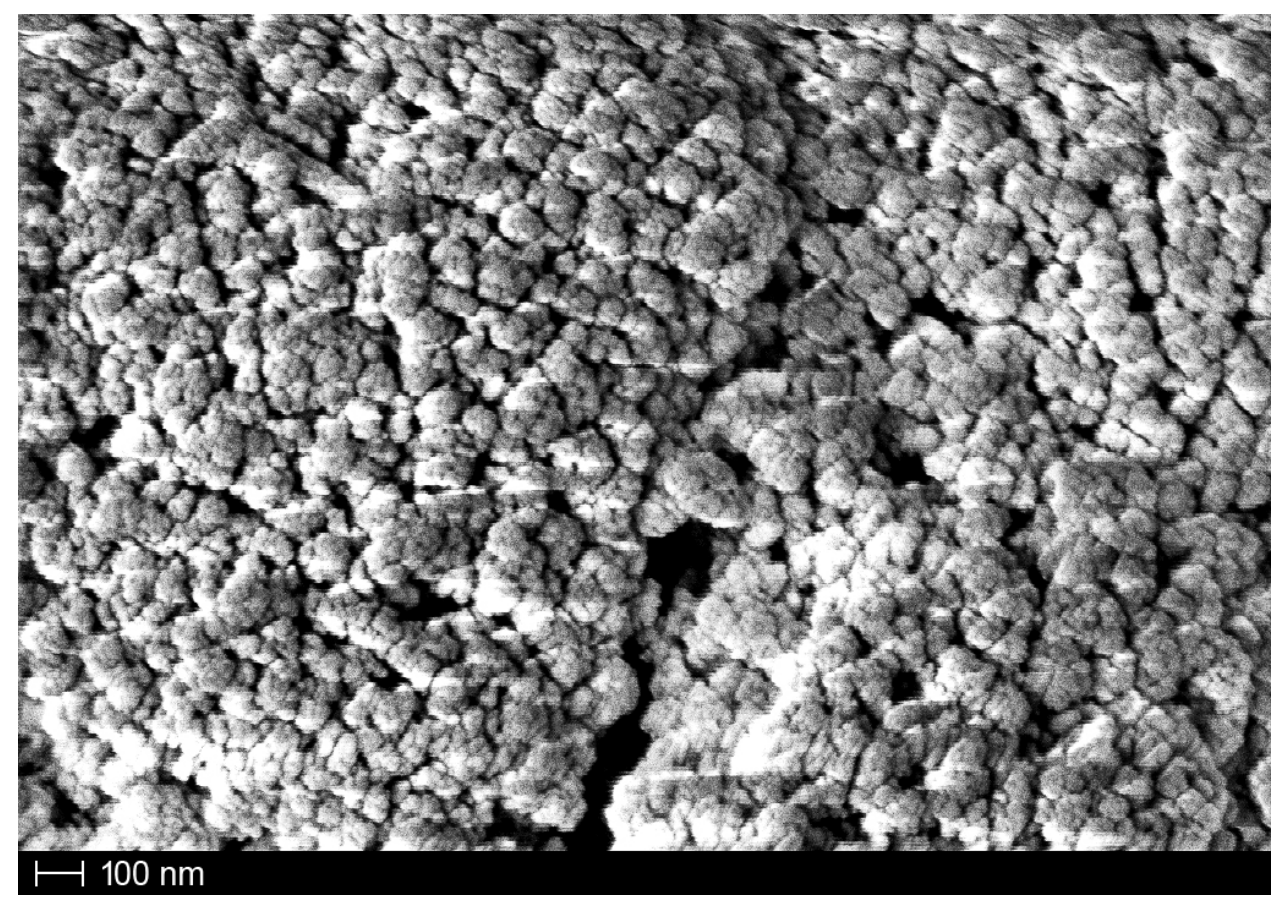

Figure 5-5. SE micrograph of silica-polystyrene hybrid 5-2.

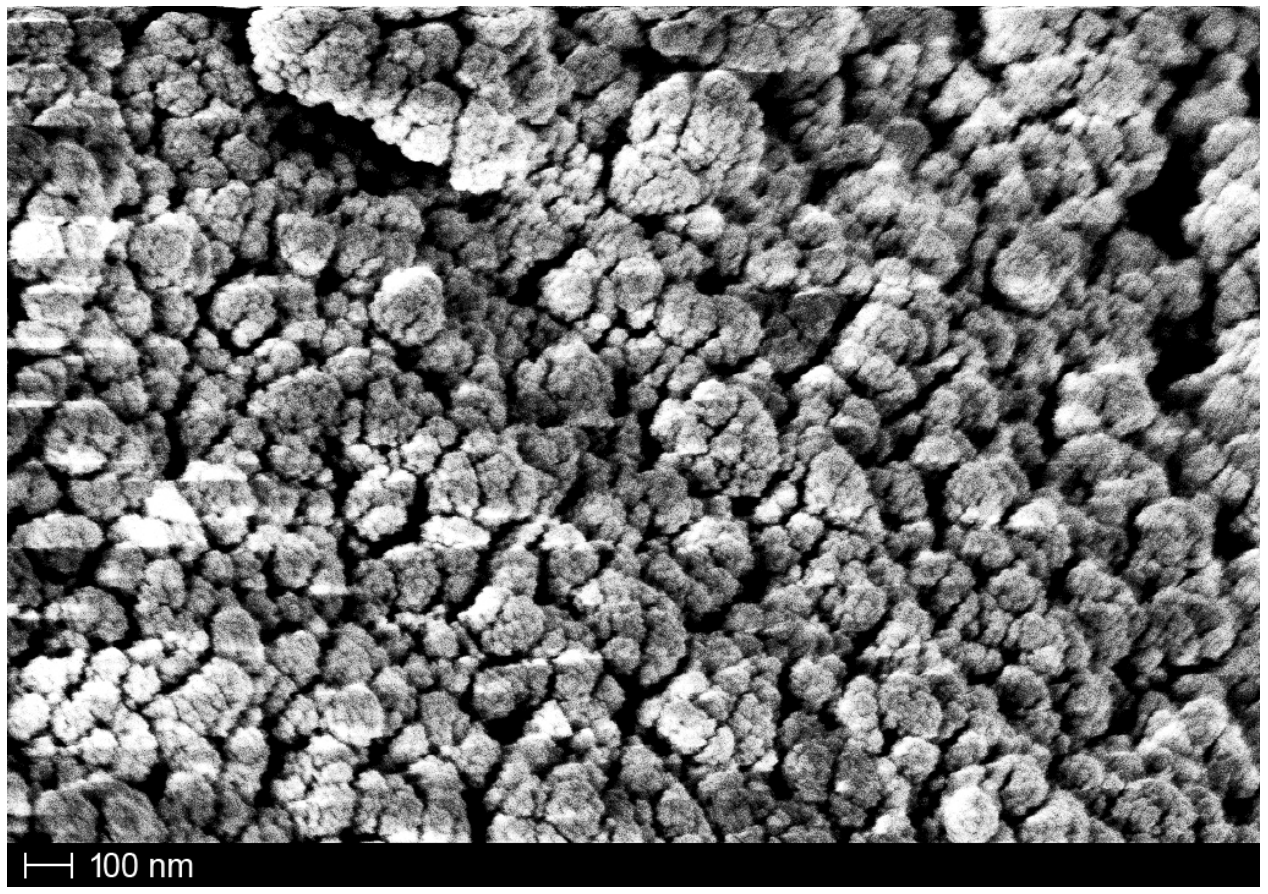

Figure 5-6. SE micrograph of silica-polystyrene hybrid 5-3. 


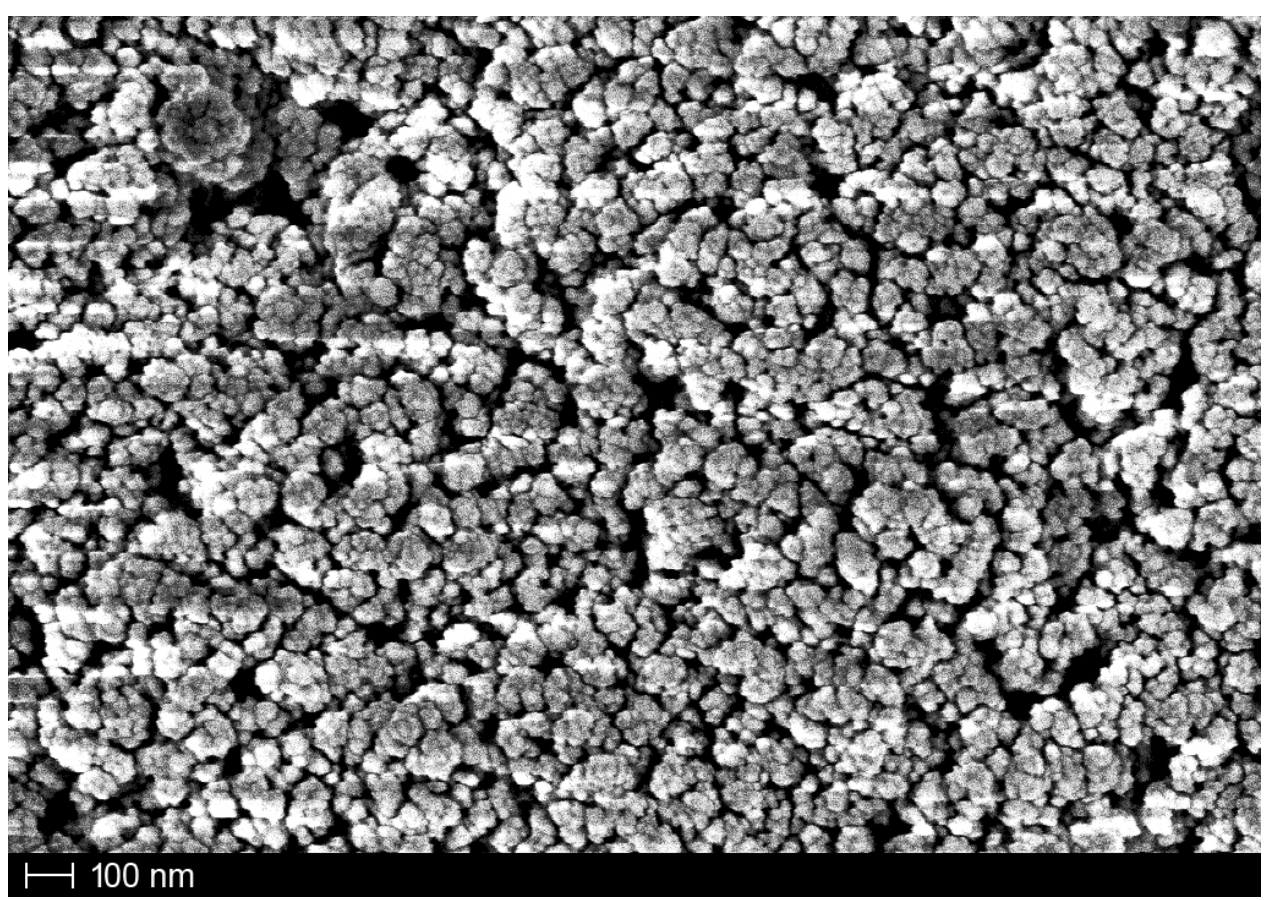

Figure 5-7. SE micrograph of silica-polystyrene hybrid 5-4.

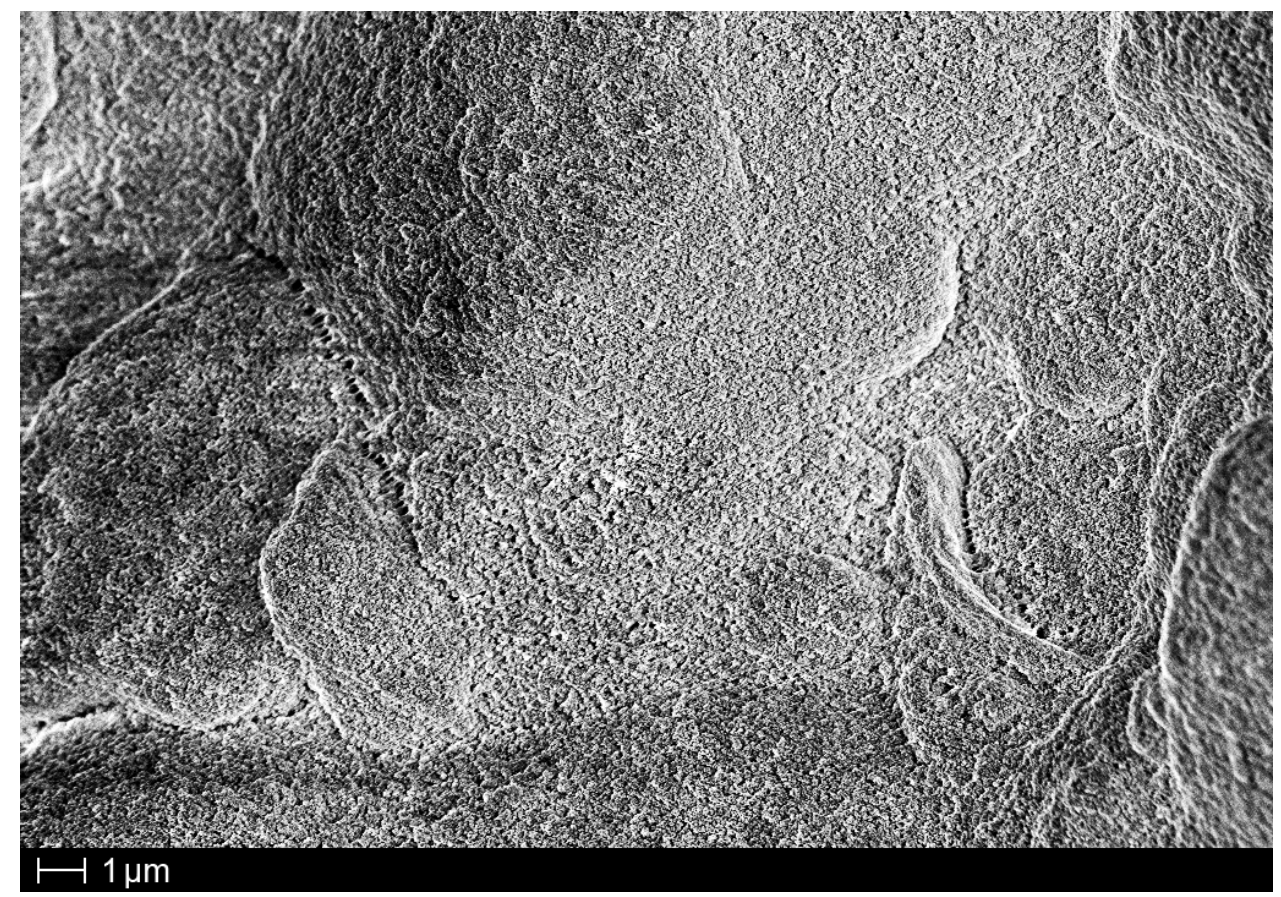

Figure 5-8. Overview SE micrograph of silica-polystyrene hybrid 5-4. Some cracks in the surface appear to be agglutinated. 


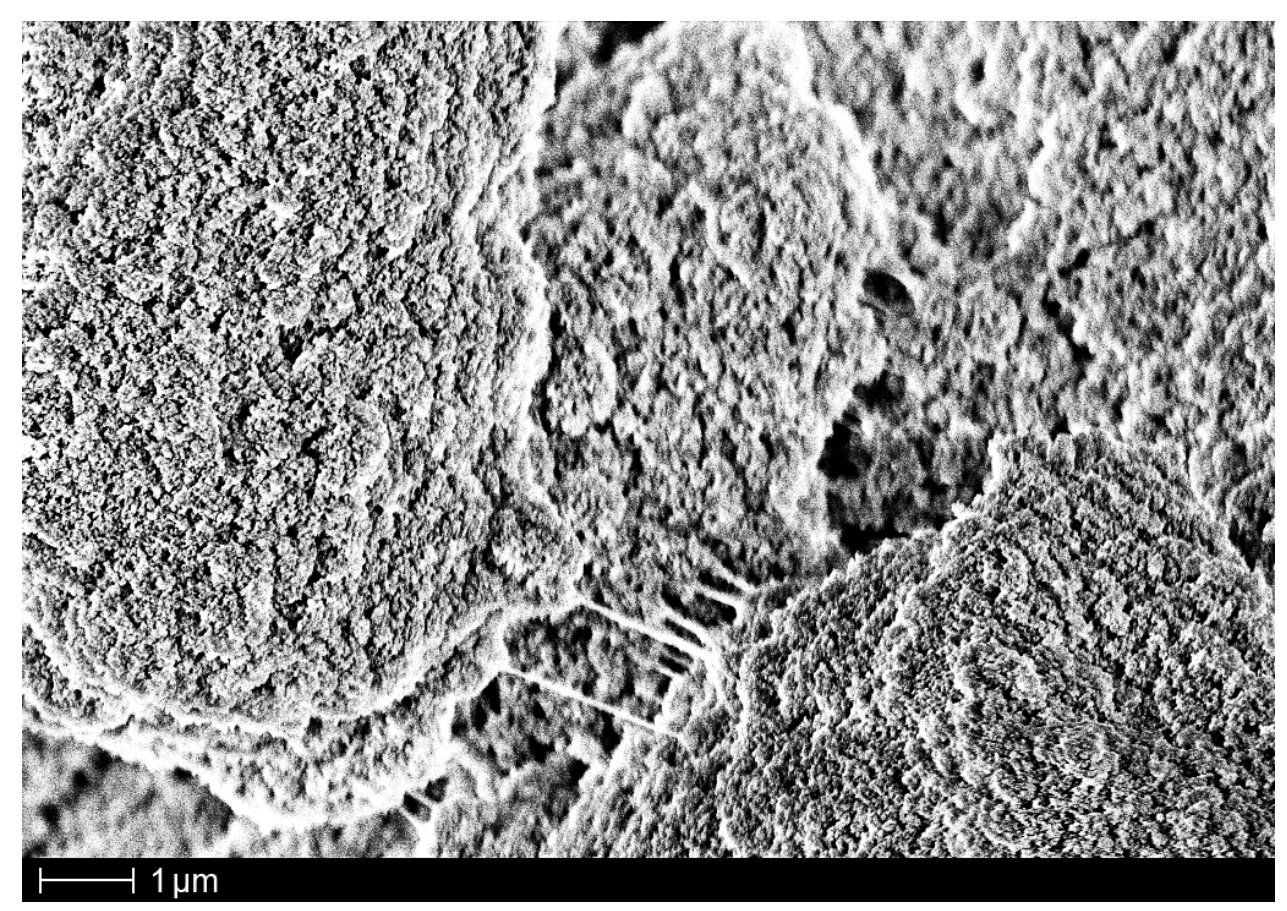

Figure 5-9. Magnified SE micrograph of glued crack in silica-polystyrene hybrid 5-4.

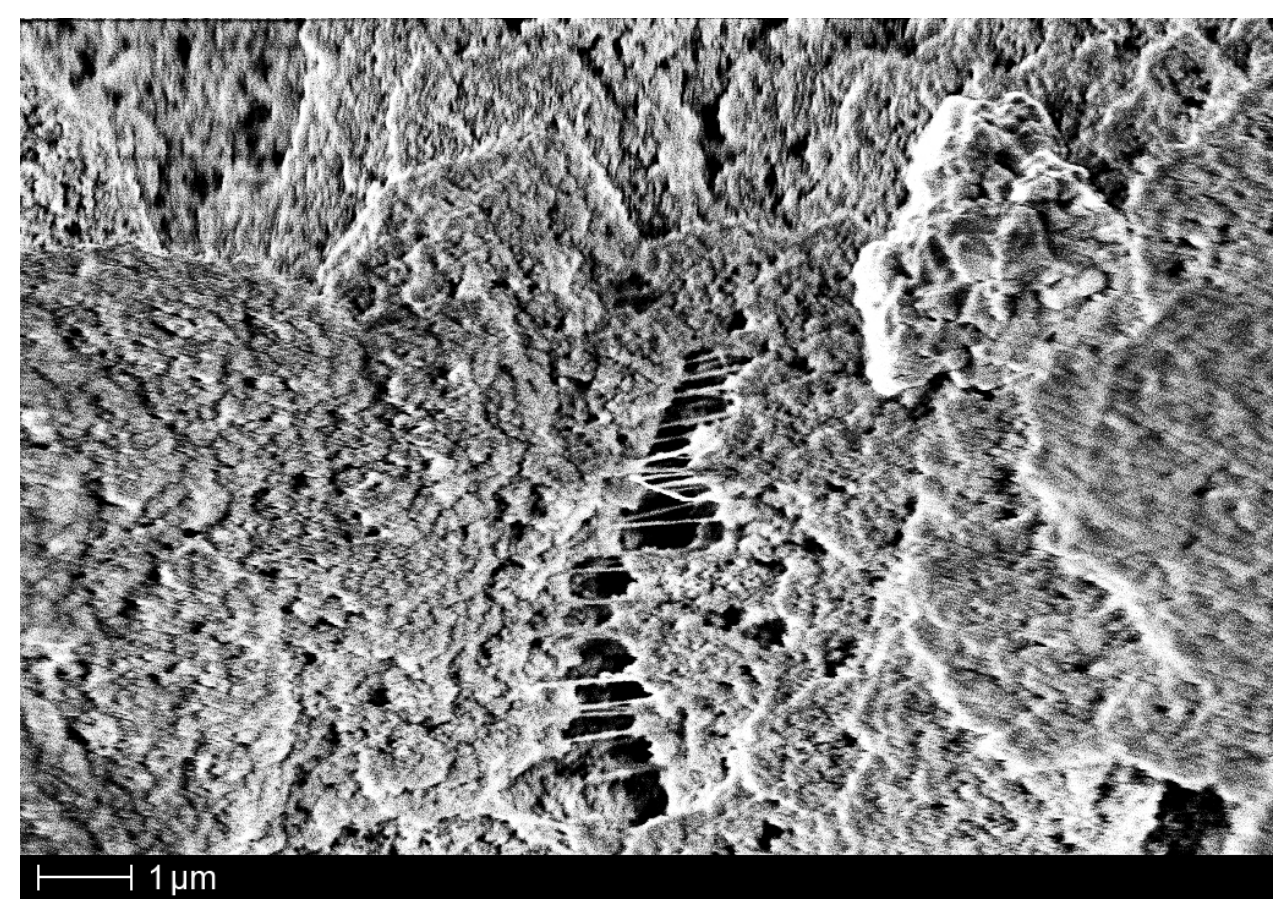

Figure 5-10. Magnified SE micrograph of glued crack in silica-polystyrene hybrid 5-4. 


\subsection{Surface-Initiated RAFT Polymerization}

For the surface-initiated RAFT polymerization of styrene, the RAFT agent cumyl dithiobenzoate (CDB, Scheme 10-1 on page 118) was dissolved in styrene before suspending the ACTA-functionalized silica. The presence of RAFT agent in the interstitial solution phase largely affects the polymerization mechanism. First of all, it should induce a living character and hence narrower MMDs as well as an increase of chain length towards higher monomer conversion for both the grafted and the free polystyrene. Secondly, the ratio of free and fixed polymer should be substantially higher compared with the conventional radical approach as the R-group of the RAFT agent almost instantly starts chain growth in solution. This way, the polymer chains that originate from the initiator and those from the R-group are both strictly separated. The initiatorderived chains are fixed to the silica particles, whereas the R-group-initiated chains are dissolved in the interstitial solution phase. This is of course a drawback for molar-mass control of the grafted polystyrene as it emerges only from the continuous initiation process of the fixed initiator and thus its MMDs are expected to be broadened compared with the RAFT-derived polystyrene in solution.

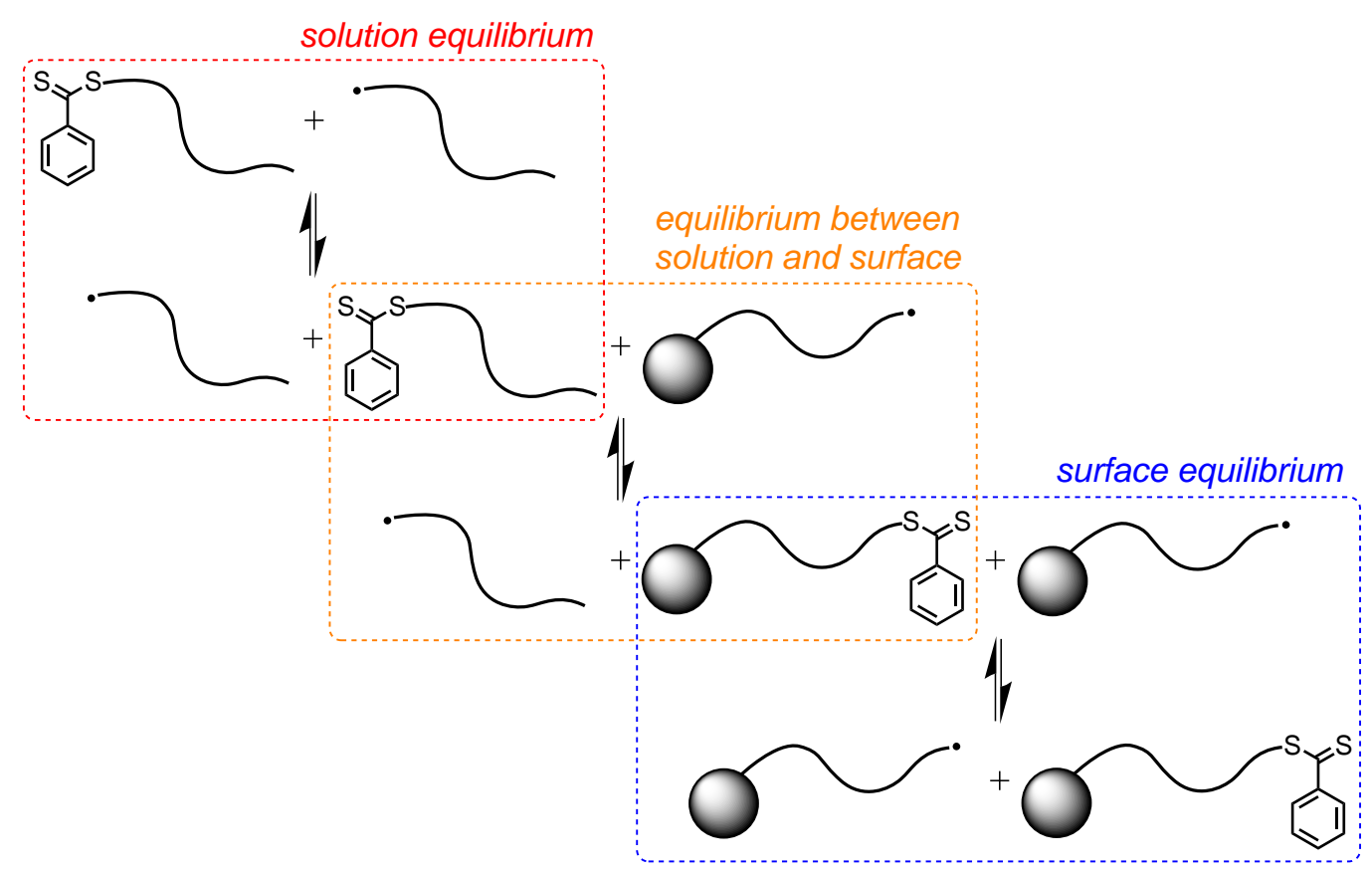

Figure 5-11. RAFT main equilibria that are expected to occur during surface-initiated polymerization with additional RAFT agent in solution. 
After the original RAFT agent is consumed, the active dithio moiety is expected to be exchanged between growing chains in solution, on the surface, as well as between the two regions. This proposed mechanism yields three RAFT main equilibria (Figure 5-11) that control the formation of free and immobilized polystyrene.

\subsubsection{Evolution of Molar Mass}

As depicted in Figure 5-12, the surface-initiated polymerization of styrene with additional free RAFT agent proceeds in a controlled fashion for both the polymer in solution and on the surface. Hence, the RAFT agent is transferred to the surface via the proposed equilibrium reactions (Figure 5-11). The molar masses of both types of polystyrene are in good agreement with the theoretical molar mass and deviate to smaller values beyond monomer conversions of about $20 \%$. The PDI values as well as the molar masses of the grafted polymer are slightly higher compared with the R-group-derived polymer (Table 5-2), but nonetheless indicate a controlled radical polymerization.

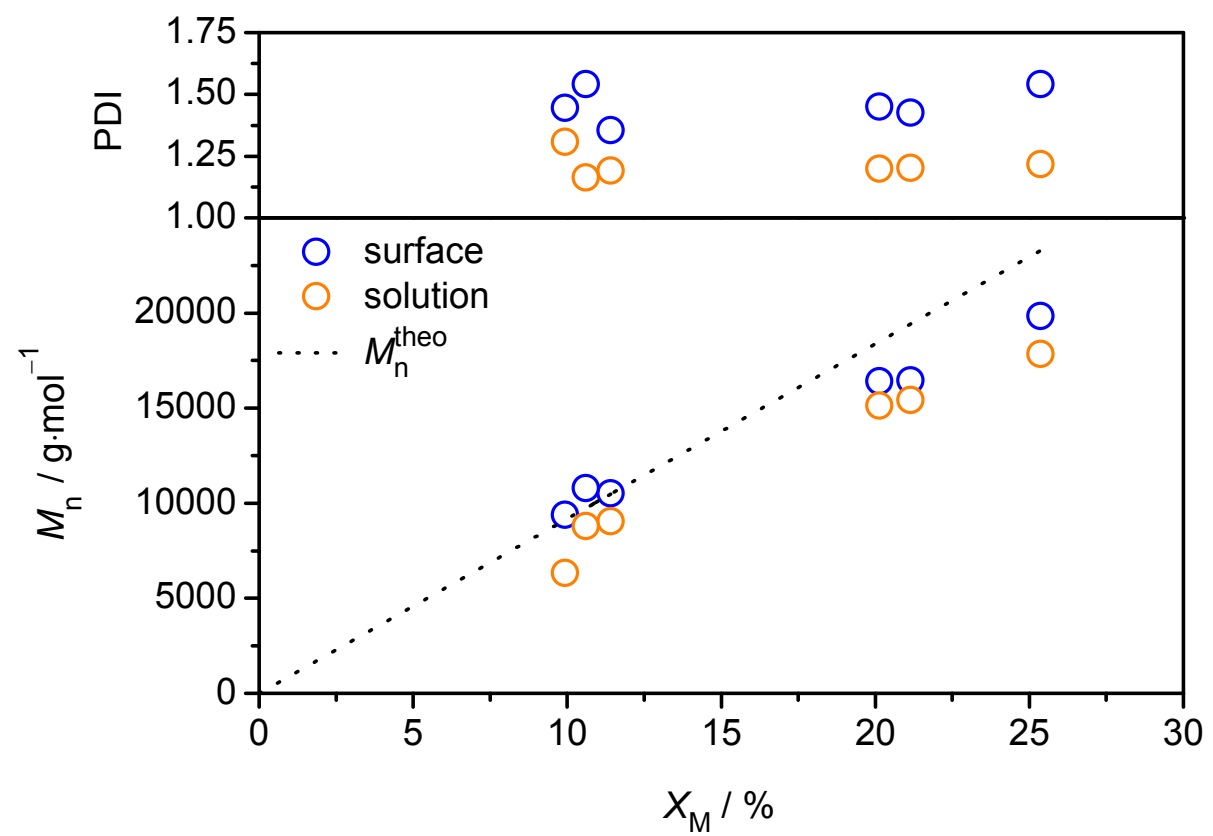

Figure 5-12. Evolution of experimental and theoretical number-average molar masses $M_{\mathrm{n}}$ and PDIs with total monomer conversion $X_{M}$ for the surface-initiated RAFT polymerization of styrene $\left(8.36 \mathrm{mmol} \cdot \mathrm{L}^{-1}\right)$ initiated by anchored ACTA $\left(3.9 \mathrm{mmol} \cdot \mathrm{L}^{-1}, 0.061 \mathrm{molecules} \cdot \mathrm{nm}^{-2}\right)$ at $60{ }^{\circ} \mathrm{C}$ and mediated by $\mathrm{CDB}$ in solution $\left(9.5 \mathrm{mmol} \cdot \mathrm{L}^{-1}\right)$. 
Table 5-2. Total monomer conversions $X_{\mathrm{M}}$, number-average molar masses $M_{\mathrm{n}}$, and PDI values of free and anchored polystyrene and calculated fractions of anchored polystyrene $f_{\text {polystyrene }}^{\text {surace }}$ for the six silica-polymer composites obtained from surface-initiated RAFT polymerization of styrene $\left(8.36 \mathrm{mmol} \cdot \mathrm{L}^{-1}\right)$ initiated by anchored ACTA $\left(3.9 \mathrm{mmol} \cdot \mathrm{L}^{-1}, 0.061 \mathrm{molecules} \cdot \mathrm{nm}^{-2}\right)$ at $60{ }^{\circ} \mathrm{C}$ and mediated by $\mathrm{CDB}$ in solution $\left(9.5 \mathrm{mmol} \cdot \mathrm{L}^{-1}\right)$.

\begin{tabular}{l|r|r|r|r|r|r}
\hline sample & $\begin{array}{c}X_{\mathrm{M}} \\
{[\%]}\end{array}$ & $\begin{array}{c}M_{\mathrm{n}}^{\text {solution }} \\
{\left[\mathrm{g} \cdot \mathrm{mol}^{-1}\right]}\end{array}$ & $\begin{array}{c}M_{\mathrm{n}}^{\text {surface }} \\
{\left[\mathrm{g} \cdot \mathrm{mol}^{-1}\right]}\end{array}$ & $\mathrm{PDI}^{\text {solution }}$ & $\mathrm{PDI}^{\text {surface }}$ & $f_{\text {polystyrene }}^{\text {surface }}$ \\
\hline $\mathbf{5 - 5}$ & 9.9 & 6329 & 9389 & 1.31 & 1.45 & 0.017 \\
$\mathbf{5 - 6}$ & 10.6 & 9052 & 10516 & 1.19 & 1.35 & 0.036 \\
$\mathbf{5 - 7}$ & 11.4 & 8788 & 10800 & 1.16 & 1.54 & 0.039 \\
$\mathbf{5 - 8}$ & 20.1 & 15122 & 16409 & 1.20 & 1.45 & 0.072 \\
$\mathbf{5 - 9}$ & 21.2 & 15427 & 16460 & 1.20 & 1.43 & 0.048 \\
$\mathbf{5 - 1 0}$ & 25.4 & 17835 & 19847 & 1.22 & 1.54 & 0.087 \\
\hline
\end{tabular}

The MMDs of the polystyrene in solution (Figure 5-13) exhibit distinct UV absorption at $330 \mathrm{~nm}$, which indicates the existence of the UV-active RAFT endgroups. In contrast, the MMDs of the polystyrene cleaved from the surface (Figure 5-14) can only be completely detected via the change in refractive index. The corresponding UV signals are rather weak, as shown exemplarily in Figure 5-15. Therefore, the initiator-derived chains do not-at the stage of SEC analysis-contain significant amounts of dithio end-groups despite their unambiguous controlled synthesis. This observation may seem surprising at first glance. However, as will be shown by the TGA in the next subchapter, the amount of living chains on the silica is fairly low and hence the UV signal may not be intensive enough to be detectable. This hypothesis is confirmed by photographs taken of sample 5-10 before and after removal of the polymer in solution. Figure 5-16 clearly shows the disappearance of the typical pink color induced by the dithio end-groups after washing with tetrahydrofurane (THF). Another factor which may hamper the UV detection of the cleaved polymer is the treatment of the silica-polystyrene hybrids with hydrofluoric acid for isolating the anchored chains, as it might induce side reactions that involve the dithio moieties.

In addition to the weak UV signals, the surface-grafted polystyrene chains also exhibit high-molar mass shoulders that are responsible for the increased molar masses and PDI values compared with the polymer formed in solution. The origin of this high-molar-mass shoulder will be discussed later in this chapter within the PREDICI ${ }^{\circledR}$ simulations. 


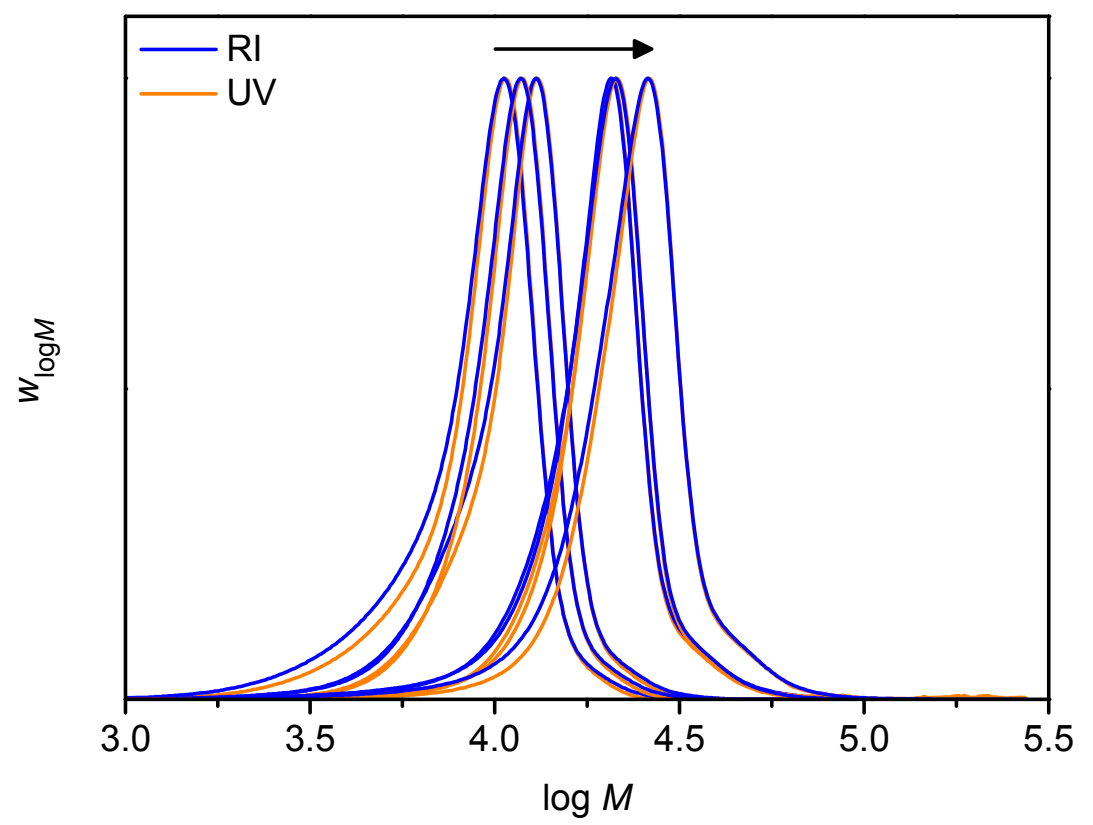

Figure 5-13. Evolution of MMD (RI and corrected UV signals at $330 \mathrm{~nm}$ ) for polystyrene formed in solution during surface-initiated RAFT polymerization of styrene $\left(8.36 \mathrm{mmol} \cdot \mathrm{L}^{-1}\right)$ initiated by anchored ACTA $\left(3.9 \mathrm{mmol} \cdot \mathrm{L}^{-1}, 0.061\right.$ molecules $\left.\cdot \mathrm{nm}^{-2}\right)$ at $60{ }^{\circ} \mathrm{C}$ and mediated by $\mathrm{CDB}$ in solution $\left(9.5 \mathrm{mmol} \cdot \mathrm{L}^{-1}\right)$.

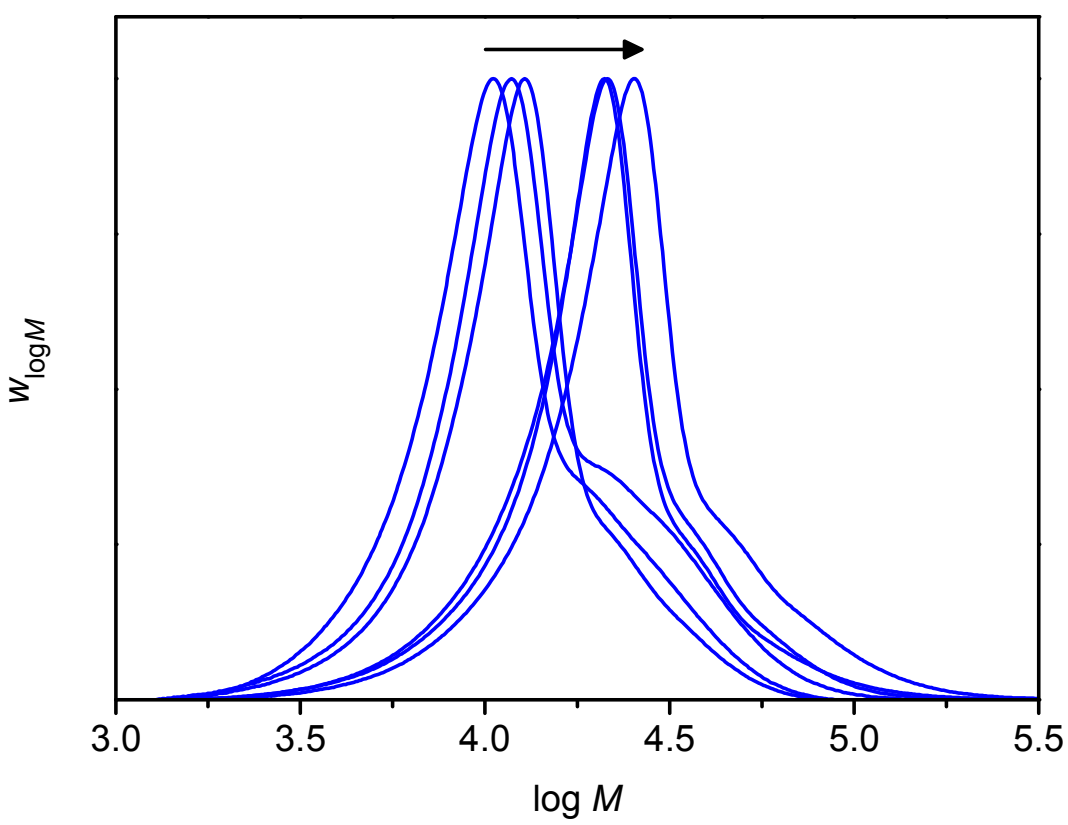

Figure 5-14. Evolution of MMD (RI signals) for polystyrene formed on the surface during surface-initiated RAFT polymerization of styrene $\left(8.36 \mathrm{mmol} \cdot \mathrm{L}^{-1}\right)$ initiated by anchored ACTA $\left(3.9 \mathrm{mmol} \cdot \mathrm{L}^{-1}, 0.061 \mathrm{molecules} \cdot \mathrm{nm}^{-2}\right)$ at $60{ }^{\circ} \mathrm{C}$ and mediated by CDB in solution $\left(9.5 \mathrm{mmol} \cdot \mathrm{L}^{-1}\right)$. 


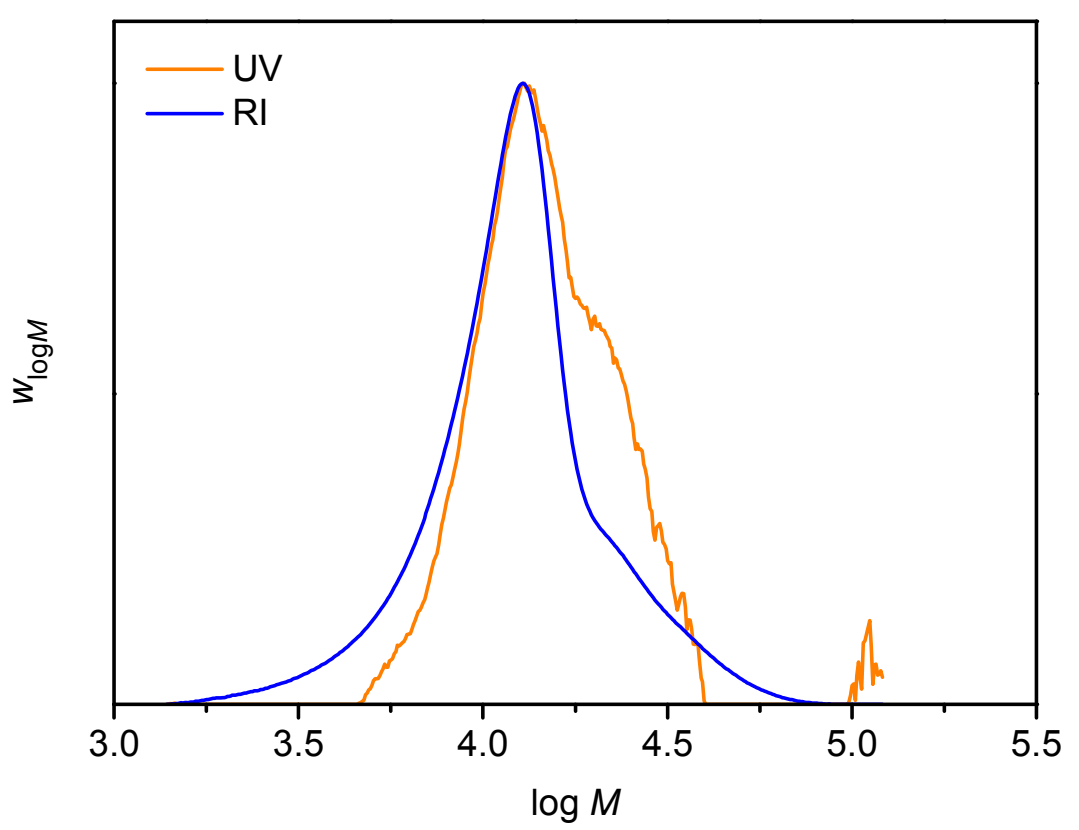

Figure 5-15. MMDs (RI and corrected UV signal at $330 \mathrm{~nm}$ ) of polystyrene formed on the surface from sample 5-7.

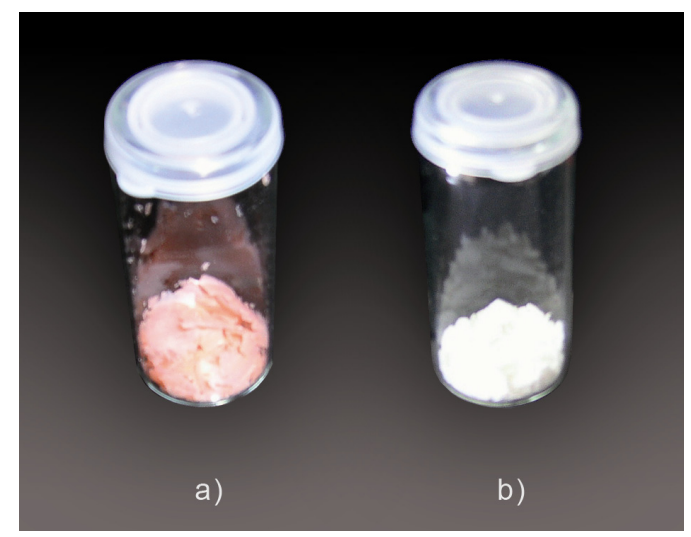

Figure 5-16. Photograph of the silica-polystyrene hybrid 5-10 before (a) and after (b) removal of the free polystyrene via washing with THF.

\subsubsection{Polymer Loading}

The TGA of the washed silica-polystyrene hybrids, depicted in Figure 5-17, indicates an increase of the grafted polymer fraction similar to the conventional radical approach described above. The most prominent difference is the smaller weight loss observed between $300{ }^{\circ} \mathrm{C}$ and $600{ }^{\circ} \mathrm{C}$. On the one hand, this can be 
attributed to the fact that the molar masses obtained from the RAFT graft polymerization are about one tenth as high as the ones from the conventional radical approach due to the controlled character of the polymerization. On the other hand, the addition of RAFT agent to the interstitial solution phase further induces a very pronounced pathway for the anchored radicals to leave the surface, which is the addition to the RAFT agent to initiate polymerization in solution. Therefore, the polymer concentration in solution is considerably higher than on the surface, which is quantitatively shown by the PREDICI ${ }^{\circledR}$ simulations presented in subchapter 5.2.4.

Samples 5-8 and 5-9 with monomer conversions of $20.1 \%$ and $21.2 \%$, respectively, display contrary weight losses, which may be due to uncertainties of the gravimetrically determined monomer conversions or the TGA itself. The latter inaccuracy may be caused by the small polymer content as the signal indeed features significant noise. The other weight losses, however, increase towards higher $X_{\mathrm{M}}$ values (Table 5-2) and reach a maximum approximate fraction of anchored polystyrene in sample 5-10 of $8.7 \%$ at $25.4 \%$ total monomer conversion.

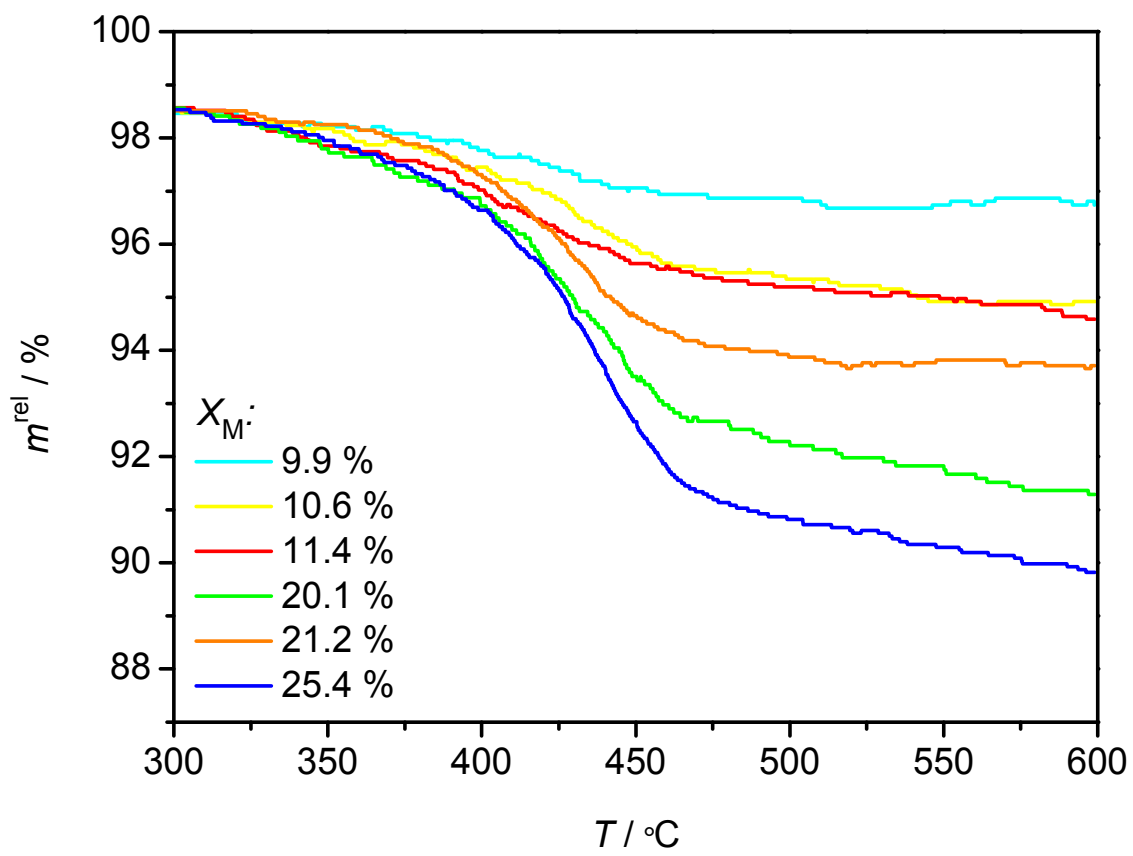

Figure 5-17. TGA curves from $300{ }^{\circ} \mathrm{C}$ to $600{ }^{\circ} \mathrm{C}$ of silica-polystyrene hybrids with different monomer conversions obtained from surface-initiated RAFT polymerization of styrene $\left(8.36 \mathrm{mmol} \cdot \mathrm{L}^{-1}\right)$ initiated by silica-anchored ACTA $\left(3.9 \mathrm{mmol} \cdot \mathrm{L}^{-1}, 0.061 \mathrm{molecules} \cdot \mathrm{nm}^{-2}\right)$ at $60{ }^{\circ} \mathrm{C}$ and mediated by CDB in solution $\left(9.5 \mathrm{mmol} \cdot \mathrm{L}^{-1}\right)$. Polymer fractions are listed in Table $5-2$. 


\subsubsection{Surface Topography}

As the amount of immobilized polymer is comparably low in the silicapolystyrene hybrids obtained from surface-initiated RAFT polymerization, the surface topography is assumed to be similar to the one of silica that is only functionalized with initiator (Figure 5-3) according to previous findings. ${ }^{[35]}$

As can be seen from SEM images of the composites obtained at lowest and highest total monomer conversion (Figure 5-18 and Figure 5-19), respectively, the differences to the ACTA-modified silica are rather small. It should be pointed out that the SE micrograph of 5-10 is not as sharp as of 5-5, hence the first one is difficult to compare. However, in spite of the low polymer loadings the surfaces appear slightly more structured and smoothed, although the differences are not as substantial as for the samples produced via the conventional radical approach where higher polystyrene fractions in the composites are achieved (subchapter 5.1.3).

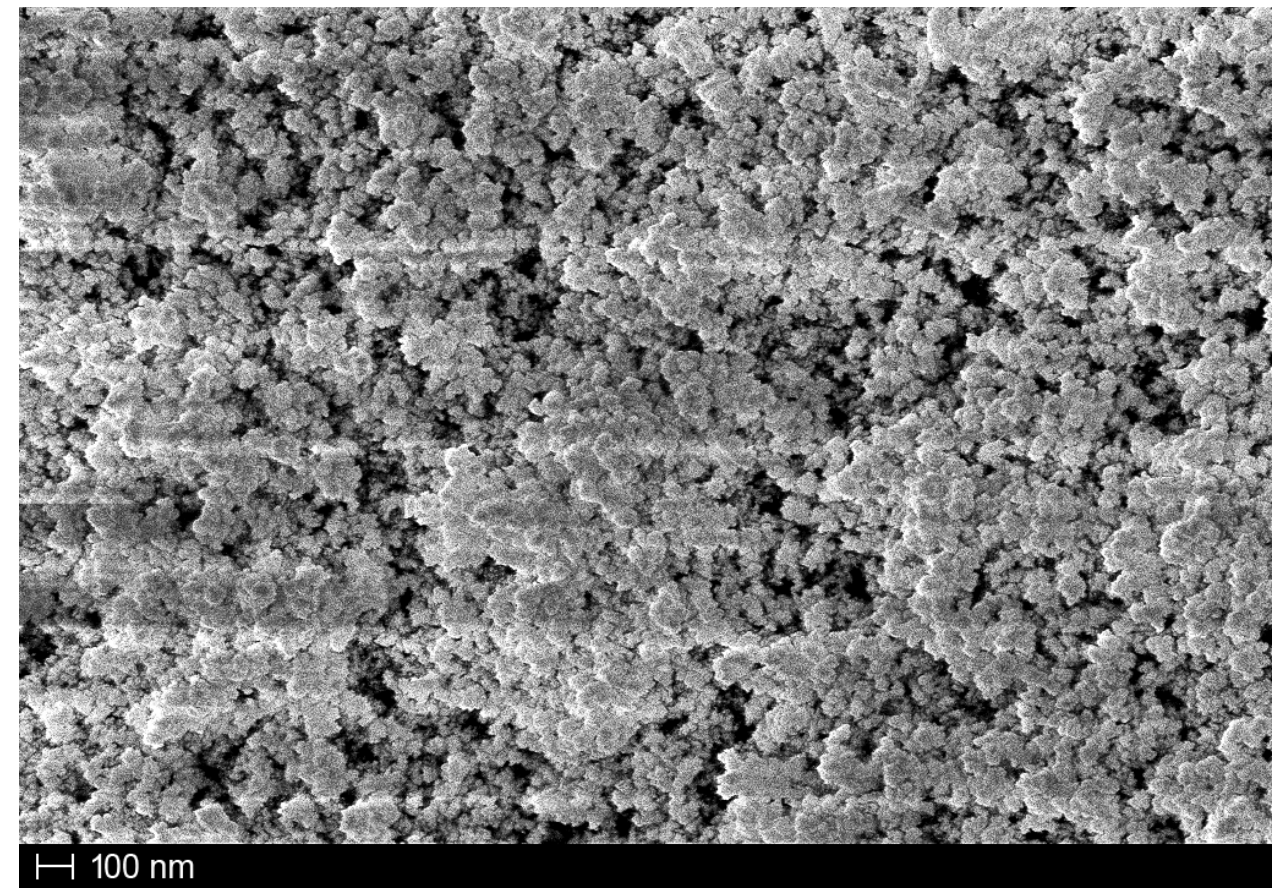

Figure 5-18. SE micrograph of silica-polystyrene hybrid 5-5, $X_{\mathrm{M}}=9.9 \%$.. 


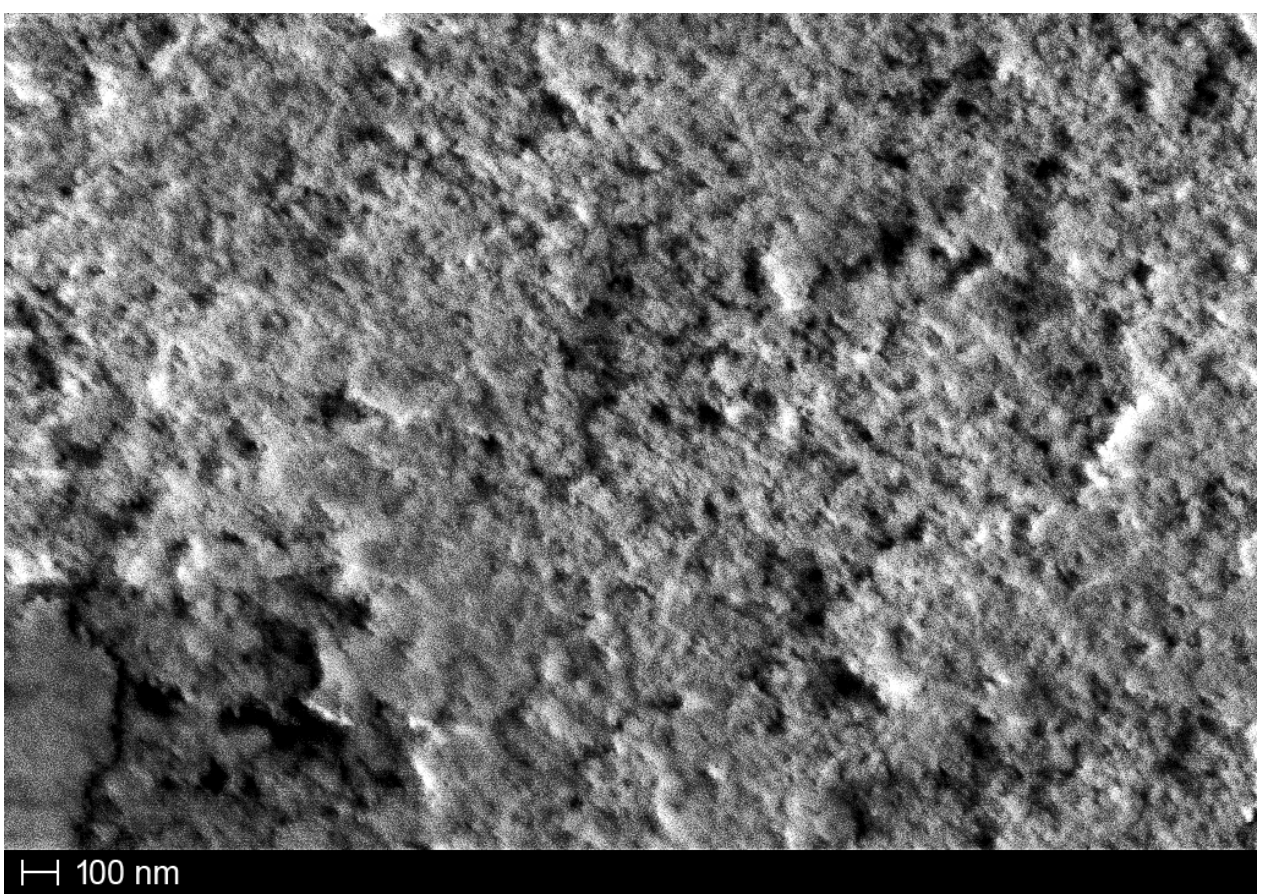

Figure 5-19. SE micrograph of silica-polystyrene hybrid 5-10, $X_{M}=25.4 \%$.

\subsubsection{Simulations}

As the surface-initiated RAFT polymerization allows for the separated analysis of the polymer formed in solution and on the surface, differences between initiator- and R-group-derived chains with respect to their chain length distributions are well-accessible. References for such investigations are scarce ${ }^{[26]}$ and in many cases, where surface-initiated polymerizations are controlled by RAFT agent in solution, the anchored polymer chains are merely assumed to exhibit similar MMDs as the polymer chains in solution. ${ }^{[28,120,121]}$ However, the experiments presented in this thesis clearly reveal distinctions between the two types of polymer. Thus a more detailed examination seems to be worthwhile to detect possible reasons for the observed differences and to gain further insights into RAFT graft polymerizations, which is the aim of the simulations presented here.

\section{Models}

The simulations were performed using the program package PREDICI ${ }^{\circledR}$. Since the surface-initiated CDB-mediated polymerization of styrene features rate retardation, two models were applied that account for the different possible origins of this phenomenon, which are the slow fragmentation and the IRT 
model. The two models differ with respect to the fragmentation rate coefficient, $k_{\beta}$, and the additional implementation of termination reactions between the RAFT intermediate species and other radicals in the system in case of the IRT model. The rate coefficients used for the models are listed in Table 5-3 and the complete input data including all chemical reactions can be found in appendix A on page 143. Furthermore, the following assumptions were used for the models:

- The rate coefficients for propagation, $k_{\mathrm{p}}$, termination, $k_{\mathrm{t}}$, and crosstermination, $k_{\mathrm{t}, \mathrm{cross}}$, are assumed to be independent on radical chain length as well as monomer conversion.

- As the initiator-specific rate coefficients $\left(k_{\mathrm{d}}, k_{\mathrm{i}}\right)$ are unknown, $k_{\mathrm{d}}$ values for AIBN were used instead and side reactions of initiator-derived radicals as well as the initiation period via reaction with monomer were neglected for simplifying the kinetic scheme.

- Unless otherwise noted, one set of the RAFT equilibrium rate coefficients, $k_{\mathrm{ad}}$ and $k_{\beta}$, was used without distinction between pre- and main equilibrium as well as the different equilibria that involve the surface radical species.

- In general, the termination rate coefficients were set equal for termination between radicals in solution $\left(k_{\mathrm{t}, \mathrm{LL}}\right)$, between radicals in solution and on the surface $\left(k_{\mathrm{t}, \mathrm{OL}}\right)$, and between two anchored radicals $\left(k_{\mathrm{t}, \mathrm{OO}}\right)$.

- In case of the IRT model, $k_{\mathrm{t}, \text { cross }}$ was assumed to be equal to $0.5 \cdot k_{\mathrm{t}}$ according to literature. ${ }^{[107]}$

Table 5-3. General parameters used in the PREDICI ${ }^{\circledR}$ simulations for the surface-initiated RAFT polymerization of styrene.

\begin{tabular}{lr}
\hline \multicolumn{1}{|c}{ parameter } & \multicolumn{1}{c}{ value } \\
\hline$k_{\mathrm{d}, \mathrm{eff}}\left[\mathrm{s}^{-1}\right]$ & $5.4 \cdot 10^{-6}$ \\
$k_{\mathrm{p}}\left[\mathrm{L} \cdot \mathrm{mol}^{-1} \cdot \mathrm{s}^{-1}\right]$ & 341 \\
$k_{\mathrm{p}, \mathrm{rein}}\left[\mathrm{L} \cdot \mathrm{mol}^{-1} \cdot \mathrm{s}^{-1}\right]$ & 341 \\
$k_{\mathrm{t}}\left[\mathrm{L} \cdot \mathrm{mol}^{-1} \cdot \mathrm{s}^{-1}\right]$ & $1.08 \cdot 10^{-8}$ \\
$k_{\mathrm{ad}}\left[\mathrm{L} \cdot \mathrm{mol}^{-1} \cdot \mathrm{s}^{-1}\right]$ & $5 \cdot 10^{5}$ \\
$k_{\beta}\left[\mathrm{s}^{-1}\right](\mathrm{slow}$ fragmentation model $)$ & $3 \cdot 10^{-2}$ \\
$k_{\beta}\left[\mathrm{s}^{-1}\right](\mathrm{IRT}$ model $)$ & $1 \cdot 10^{4}$ \\
$c_{\text {AIBN }}^{0}\left[\mathrm{~mol} \cdot \mathrm{L}^{-1}\right]$ & $3.9 \cdot 10^{-3}$ \\
$c_{\mathrm{RAFT}}^{0}\left[\mathrm{~mol} \cdot \mathrm{L}^{-1}\right]$ & $9.5 \cdot 10^{-3}$ \\
$c_{\text {monomer }}^{0}\left[\mathrm{~mol} \cdot \mathrm{L}^{-1}\right]$ & 8.36 \\
\hline
\end{tabular}




\section{General Characteristics}

The main feature of the developed models is the strict separation of initiatorand RAFT-derived polymer chains similar to the above-described surfaceinitiated polymerization of styrene. The anchored polystyrene originates only from radicals that are generated by the fixed initiator, whereas all polymer chains in solution arise from reinitiation of the R-group radical cleaved from the RAFT agent after attack of fixed radicals, as depicted in Scheme 5-1. Although this model is developed in order to simulate MMDs for the surfaceinitiated RAFT polymerization of styrene, it can be applied to any RAFT polymerization that is initiated by a thermal initiator for studying the initiatorderived and R-group-derived chains separately. However, it should also be noted that this model assumes a homogeneous system and thus neglects heterogeneously dispersed reagents, as for instance the silica-anchored initiator.

initiation of chains on surface:

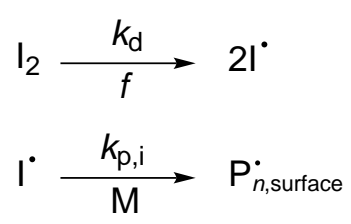

initiation of chains in solution:

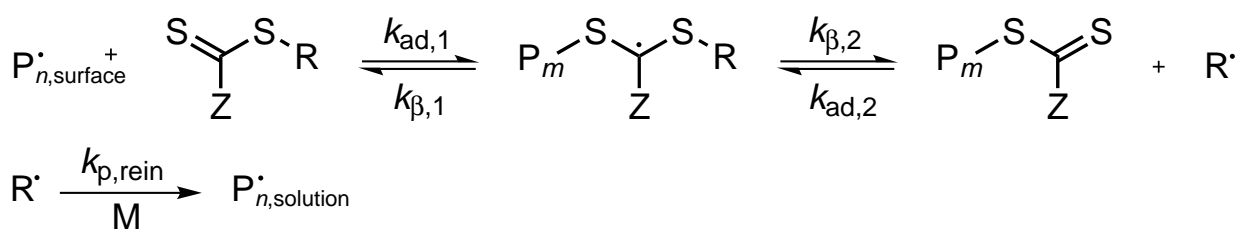

Scheme 5-1. The origins of the polymer on the surface and in solution by which the two types of polymers are separated in the PREDICI ${ }^{\circledR}$ simulations.

As a result of the detachment of fixed and free polymer, the concentration profiles of both species differ greatly, as shown exemplarily for the IRT model in Figure 5-20. The amount of grafted chains increases with time because of the continuous initiator decay, while the number of free chains increases instantly at the beginning due to the fast initiation of the R-group and then slowly decreases because of intermediate radical termination. For the slow fragmentation model the concentration of the free RAFT polymer reaches a constant concentration beyond stationary reaction conditions. In addition, the concentration of polystyrene in solution is about four times higher than the 
concentration of polystyrene on the surface after $77400 \mathrm{~s}$ reaction time, which is in alignment with the experimental TGA data presented above.

\section{Comparison of grafted and free polymer}

In the following, the MMDs of the grafted and free polystyrene after $77400 \mathrm{~s}$ of polymerization at $60{ }^{\circ} \mathrm{C}$ are simulated using both models and compared with the experimental MMDs. Subsequently, the influence of selected parameters is examined for achieving the highest possible agreement between theoretical and experimental MMDs.

The simulated MMDs for the living and dead polymer (produced by termination via combination with the rate coefficient $k_{\mathrm{t}}$ ) in solution and on the surface (Figure 5-21 and Figure 5-22) already deliver the source for the high-molar-mass shoulder of the grafted polystyrene. The amount of dead polymer on the surface that is formed by termination of a fixed and a free macroradicalyielding a dead single-anchored polymer chain-is higher than the amount of free dead polymer relative to the corresponding living polymer. Hence the dead fraction is well-visible in case of the anchored polymer, but can hardly be detected for the polystyrene in solution. The amount of termination product formed by reaction of two grafted radicals is too low to be measured according to simulation because of the low radical concentration on the surface.

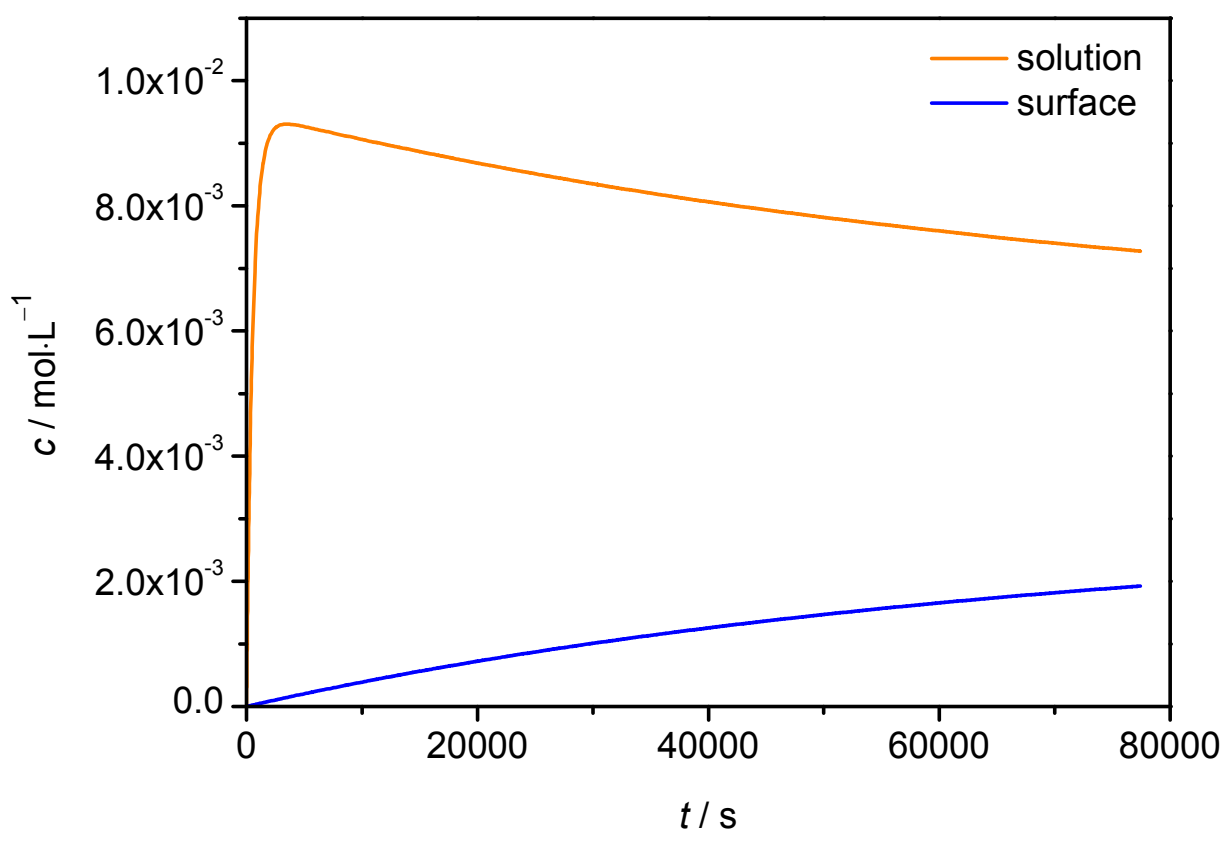

Figure 5-20. Concentration profiles of the living polystyrene on the surface and in solution simulated in PREDICI ${ }^{\circledR}$ with the IRT model for the surface-initiated RAFT polymerizaton of styrene. General reaction parameters are given in Table 5-3. 


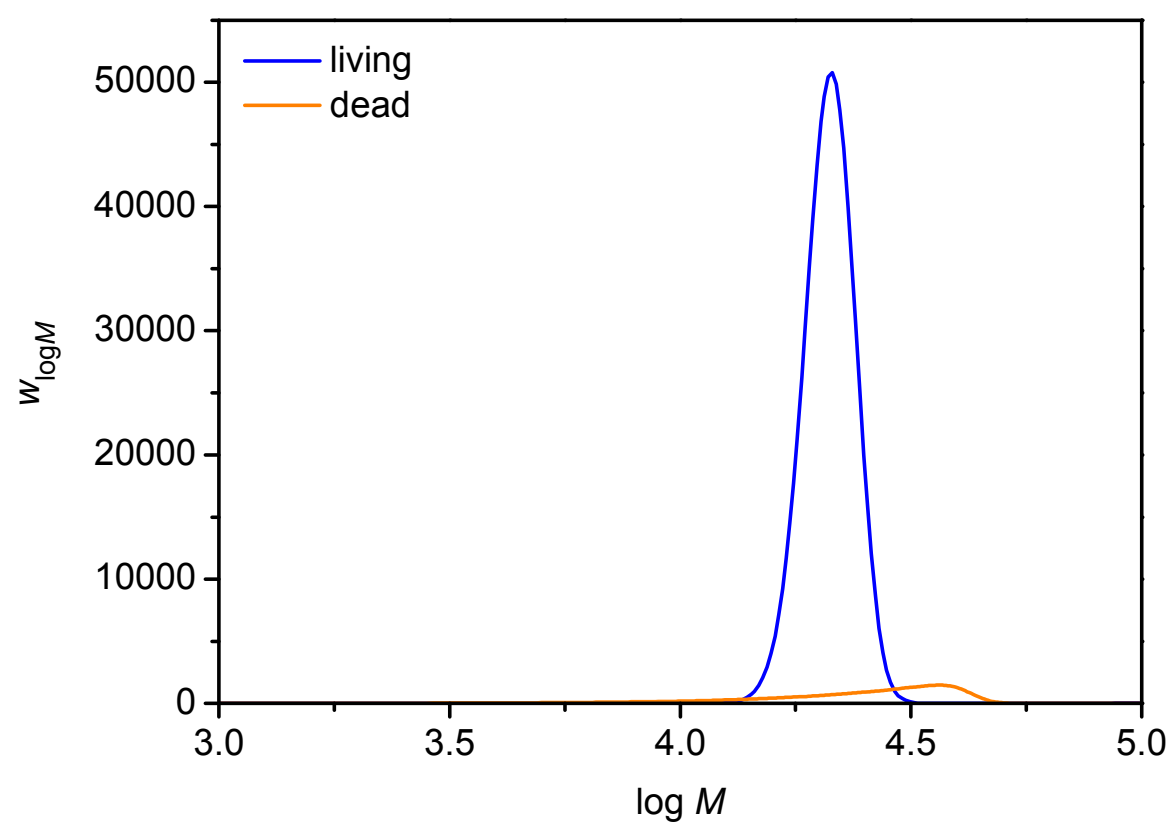

Figure 5-21. MMDs of living and dead polymer in solution simulated in PREDICI $^{\circledR}$ with the IRT model for the surface-initiated RAFT polymerization of styrene. General reaction parameters are given in Table 5-3.

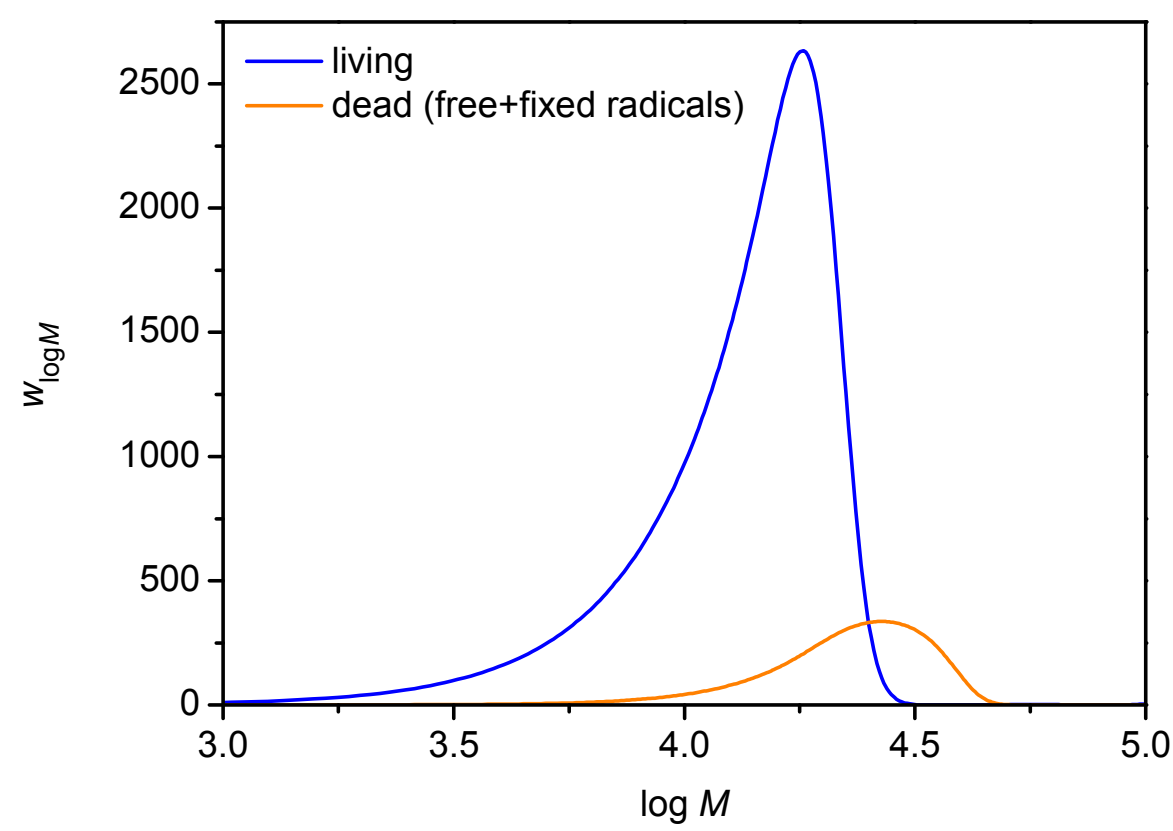

Figure 5-22. MMDs of living and dead polymer on the surface simulated in PREDICI ${ }^{\circledR}$ with the IRT model for the surface-initiated RAFT polymerization of styrene. General reaction parameters are given in Table 5-3. 
In order to directly compare the MMDs of free and anchored polymer, the simulated MMDs of living and dead polymer are added together for both types of polystyrene and then normalized. The experimental MMDs are taken from Sample 5-6 polymerized after $77600 \mathrm{~s}$ (Figure 5-23), which show that both distributions nearly have identical molar masses at the peak maximum and that the MMD of the anchored polymer is broadened and exhibits the high-molarmass shoulder which arises from dead material.

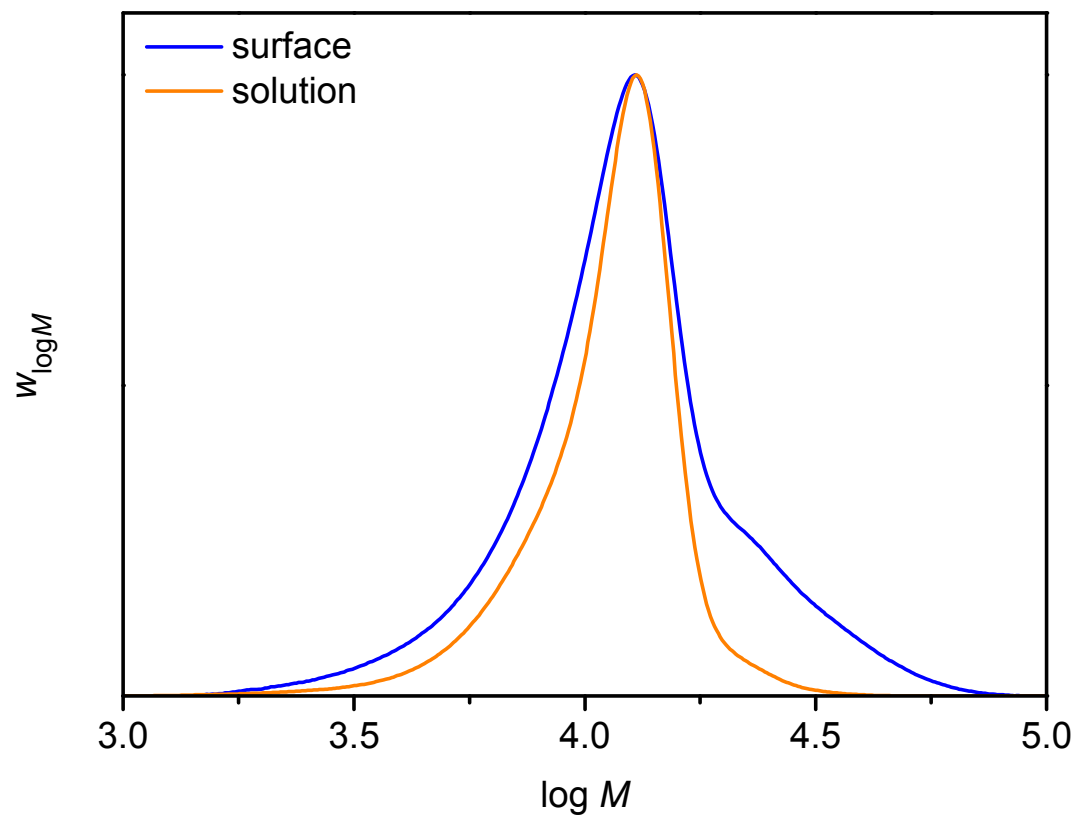

Figure 5-23. Experimental MMDs (RI signal) of free and grafted polymer obtained after $77600 \mathrm{~s}$ of surface-initiated RAFT polymerization of styrene $\left(8.36 \mathrm{mmol} \cdot \mathrm{L}^{-1}\right)$ initiated by anchored ACTA $\left(3.9 \mathrm{mmol} \cdot \mathrm{L}^{-1}\right)$ at $60{ }^{\circ} \mathrm{C}$ and mediated by CDB in solution $\left(9.5 \mathrm{mmol} \cdot \mathrm{L}^{-1}\right)$.

The simulated MMDs obtained from both models using standard parameters, shown in Figure 5-24 and Figure 5-25, yield quite different results. In case of the IRT model the grafted polystyrene displays a much broader MMD that is shifted to lower molar masses compared to the polymer in solution. The slow fragmentation model, in turn, delivers fewer differences between the two types of polystyrene as the MMD of the anchored polystyrene is only slightly shifted to smaller masses, although it is also broadened due to initiator decay. Both models feature a high-molar-mass shoulder and agree in the broadened distribution of the grafted polymer. However, distinct differences to the experimental MMDs are visible. The MMDs of the anchored and free polystyrene do not overlap at intermediate masses and the low-molar-mass 
shoulder of the free polystyrene is missing. In addition to that, the high-molarmass shoulder of the anchored polystyrene, assigned to the termination product of a free and a grafted macroradical, is less pronounced in the simulated MMDs, especially for the MMDs obtained by application of the slow fragmentation model.

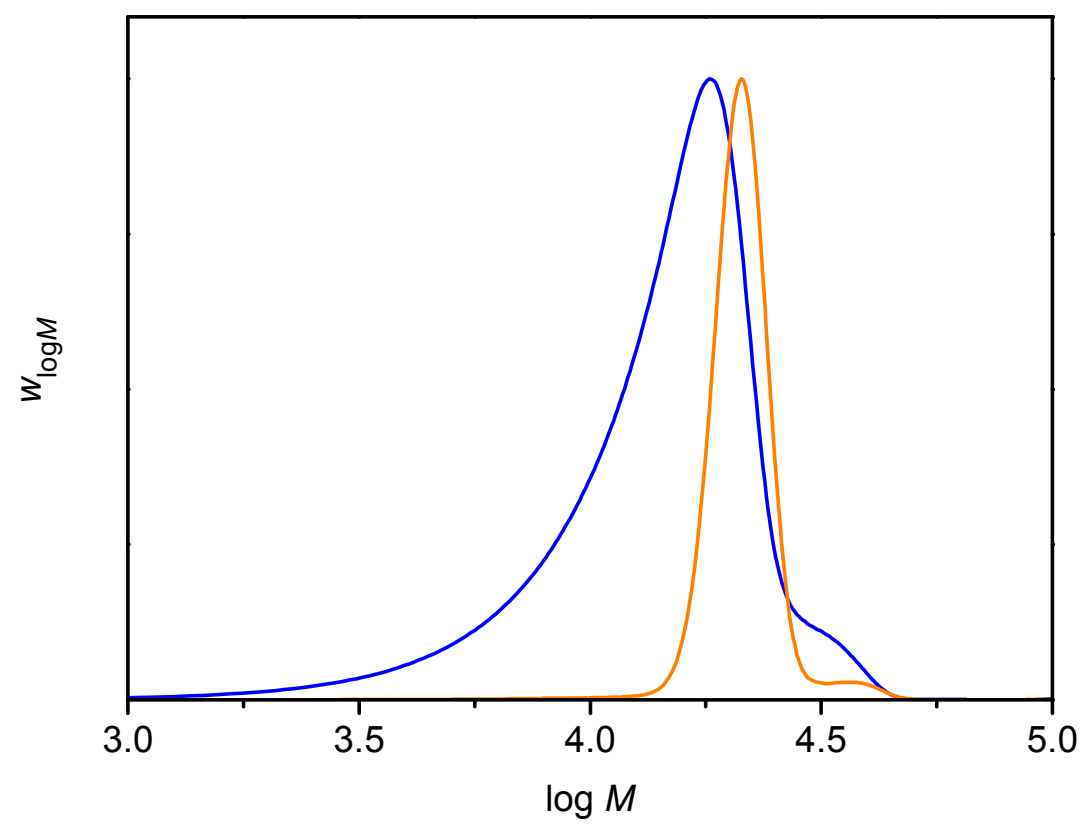

Figure 5-24. MMDs of anchored and free polymer simulated in PREDICI ${ }^{\circledR}$ with the IRT model for the surface-initiated RAFT polymerization of styrene. General reaction parameters are given in Table 5-3.

Therefore, selected rate coefficients, whose value may be different compared with standard conditions, were varied in order to shed light on possible deviations present in the surface-initiated polymerization system.

The termination rate coefficient between anchored radicals, $k_{\mathrm{t}, \mathrm{OO}}$, was altered, as earlier experiments revealed significant differences in the termination process on solid surfaces. ${ }^{[33]}$ However, neither of the two models display a noticeable influence to changes of this parameter (Table 5-4).

Other rate coefficients that may very well be different from standard values are the addition rate coefficients of the RAFT equilibria, specifically the ones describing the rate of addition in the pre-equilibrium on the surface, $k_{\text {ad,pre }}$, as well as the main equilibria that involve grafted species, $k_{\mathrm{ad}, \mathrm{OL}}$ and $k_{\mathrm{ad}, \mathrm{OO}}$. These rate coefficients may display variations from standard values as in all cases fixed species are involved, which are hindered in motion and hence the 
effective addition rate coefficients may be decreased. Furthermore, these reactions also contain the diffusion of reagents from the interstitial solution phase to the surface, which may also cause a smaller overall reaction rate.

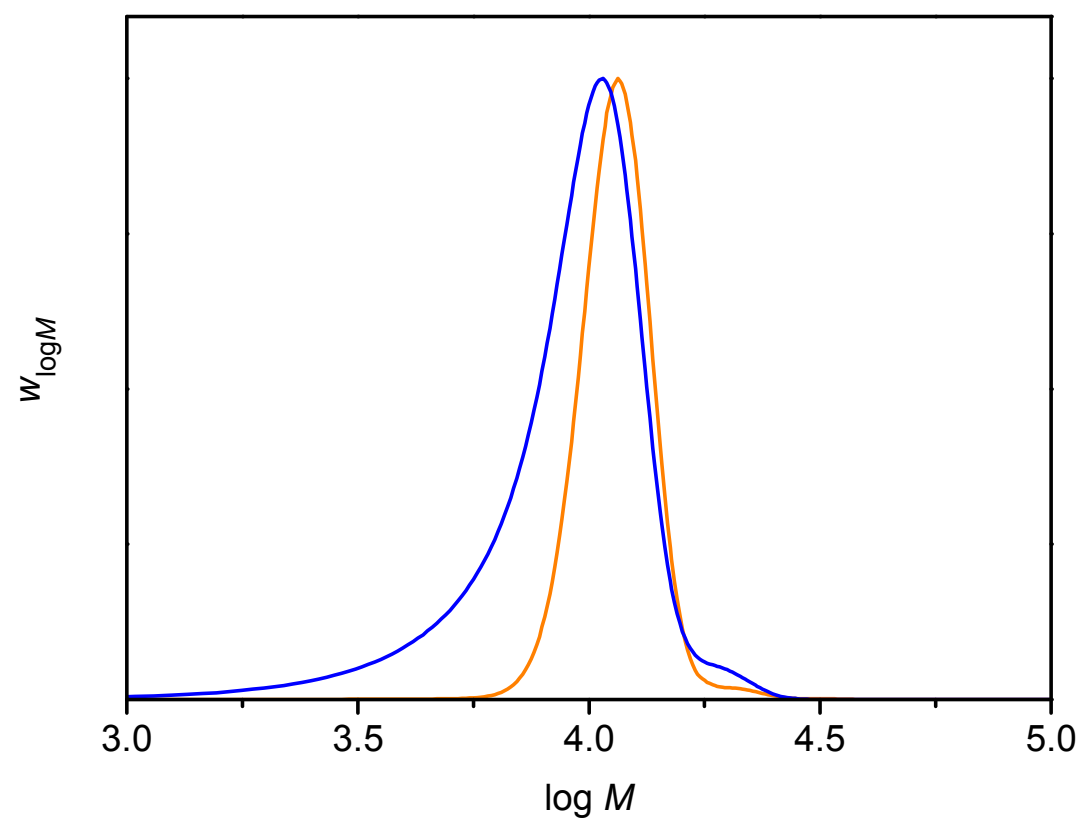

Figure 5-25. MMDs of anchored and free polymer simulated in PREDICI ${ }^{\circledR}$ with the slow fragmentation model for the surface-initiated RAFT polymerization of styrene. General reaction parameters are given in Table 5-3.

As depicted in Table 5-4, changes in the addition rate coefficient of the preequilibrium does not significantly affect the resulting MMDs. However, variation of the addition rate coefficients of the main equilibria leads to a change in the simulated MMDs. In case of the IRT model, only the rate coefficient for addition between grafted and free reactants displays significant impact on the MMDs, especially on the MMD of the fixed polystyrene. By decreasing $k_{\mathrm{ad}, \mathrm{OL}}$ by a factor of 10, the MMD significantly broadens and shifts towards higher molar masses, whereas the MMD for the free polymer stays nearly constant (Figure 5-26). This effect seems to arise from the fact that the grafted chains do not participate in the main equilibria to full extent since the transfer of RAFT moieties to the surface is diminished. This, on the other hand, causes a corruption of control for the polymerization on the surface. 
Table 5-4. Influence of selected rate coefficients on the MMDs simulated in PREDICI ${ }^{\circledR}$ for both applied models for the surface-initiated RAFT polymerization of styrene.

\begin{tabular}{l|l|l}
\hline $\begin{array}{c}\text { rate } \\
\text { coefficient }\end{array}$ & \multicolumn{1}{c}{$\begin{array}{c}\text { IRT } \\
\text { model }\end{array}$} & \multicolumn{1}{c}{$\begin{array}{c}\text { slow fragmentation } \\
\text { model }\end{array}$} \\
\hline$k_{\text {ad,pre }}$ & none & none \\
$k_{\mathrm{t}, \mathrm{OO}}$ & none & none \\
$k_{\mathrm{ad}, \mathrm{OO}}$ & none & small \\
$k_{\mathrm{ad}, \mathrm{OL}}$ & big & big \\
\hline
\end{tabular}

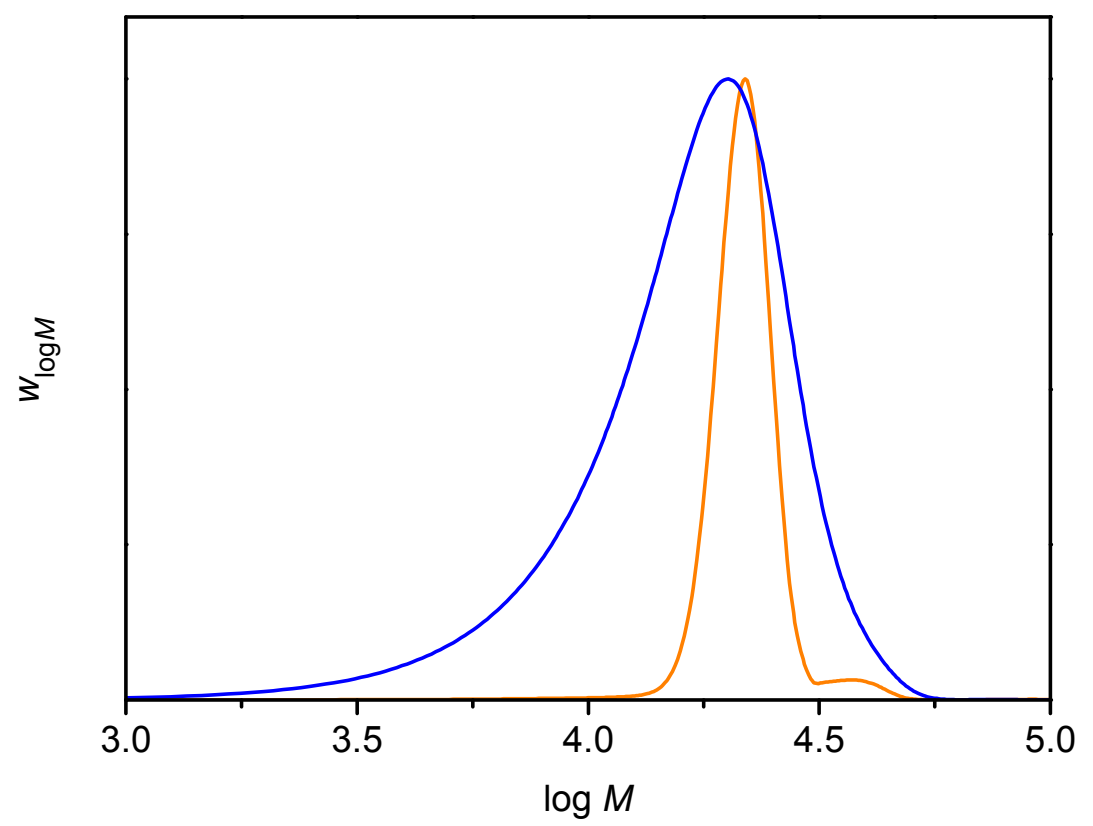

Figure 5-26. MMDs of anchored and free polymer simulated in PREDICI ${ }^{\circledR}$ with the IRT model for the surface-initiated RAFT polymerization of styrene with $k_{\mathrm{ad}, \mathrm{OL}}=0.1 \cdot k_{\mathrm{ad}}$. General reaction parameters are given in Table 5-3.

Alterations of $k_{\mathrm{ad}, \mathrm{OO}}$ and $k_{\mathrm{ad}, \mathrm{OL}}$ for the slow fragmentation model both affect the resulting MMDs, although the effect is only minor for the first rate coefficient. In case the main equilibrium is completely turned off on the surface $\left(k_{\mathrm{ad}, \mathrm{OO}}=0\right)$, the MMD of the anchored polystyrene is slightly shifted to higher molar masses without broadening (Figure 5-27). However, although the trend is right, the effect appears to be too small as both MMDs still do not overlap even though $k_{\mathrm{ad}, \mathrm{OO}}$ is zero. A reduction of $k_{\mathrm{ad}, \mathrm{OL}}$ by factors of 0.75 and 0.5 , illustrated 
in Figure 5-28 and Figure 5-29, results in more noticeable effects on the MMDs. In this case the MMD of the immobilized polymer is gradually shifted to higher molar masses with decreasing $k_{\mathrm{ad}, \mathrm{OL}}$ value without lack of control. A bisection of the addition rate coefficient delivers MMDs for free and grafted polystyrene that resemble the experimental MMDs fairly well in the proximity of the peak maxima. This phenomenon emerges from the partial withdrawal of anchored radicals from the RAFT equilibria and thus also from rate retardation caused by slow fragmentation in this model. Therefore, the grafted macroradicals have effectively more time to propagate, which results in higher chain lengths.

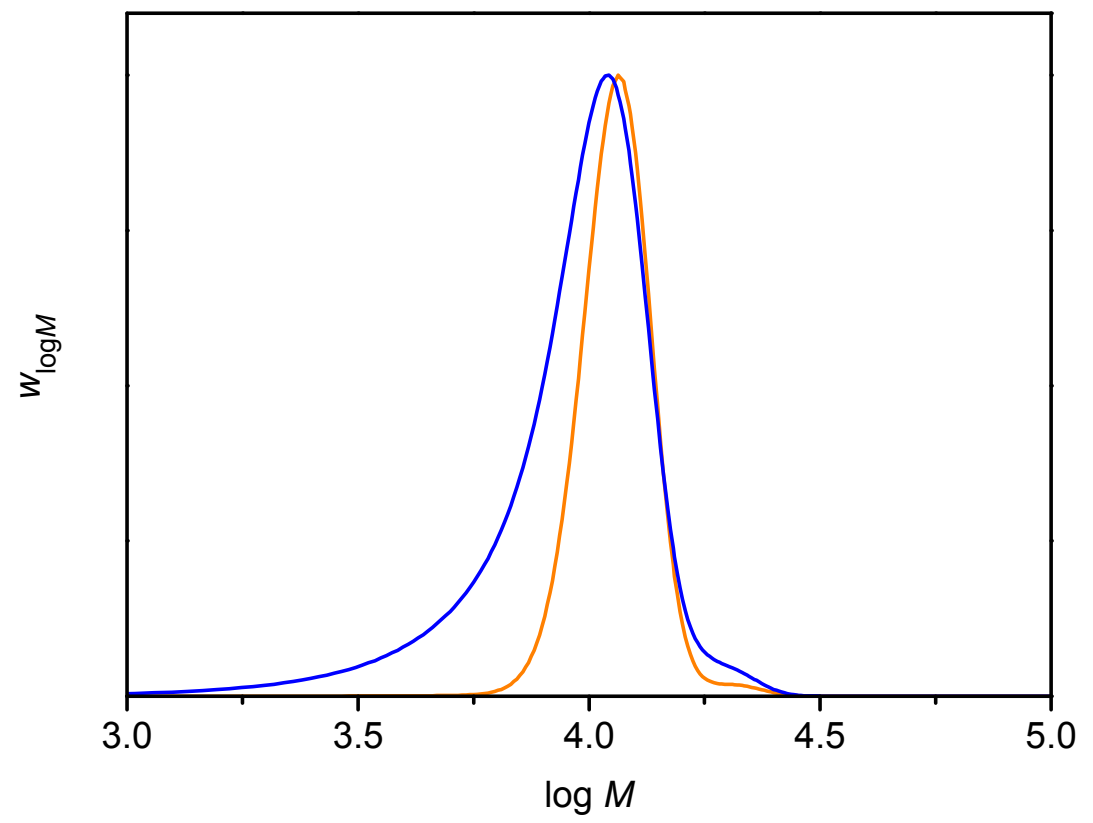

Figure 5-27. MMDs of anchored and free polymer simulated in PREDICI ${ }^{\circledR}$ with the slow fragmentation model for the surface-initiated RAFT polymerization of styrene with $k_{\mathrm{ad}, \mathrm{OO}}=0$. General reaction parameters are given in Table 5-3. 


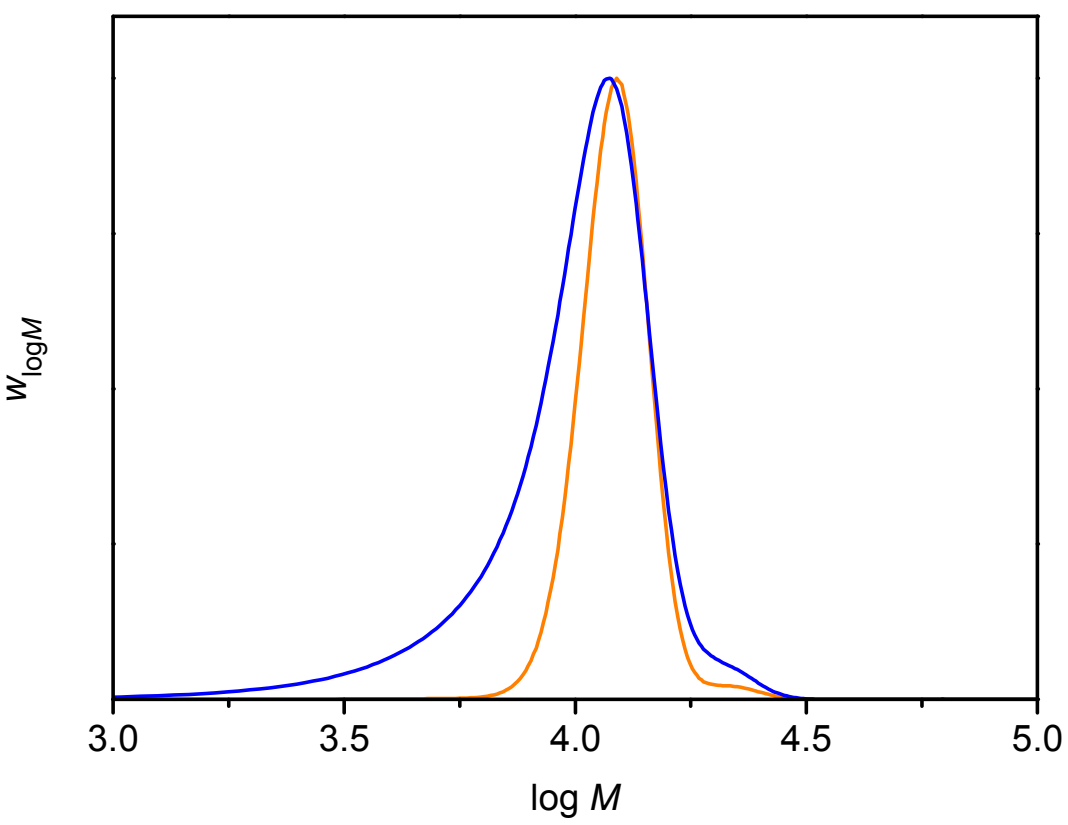

Figure 5-28. MMDs of anchored and free polymer simulated in PREDICI ${ }^{\circledR}$ with the slow fragmentation model for the surface-initiated RAFT polymerization of styrene with $k_{\mathrm{ad}, \text { OL }}=0.75 \cdot k_{\mathrm{ad}} \cdot$ General reaction parameters are given in Table 5-3.

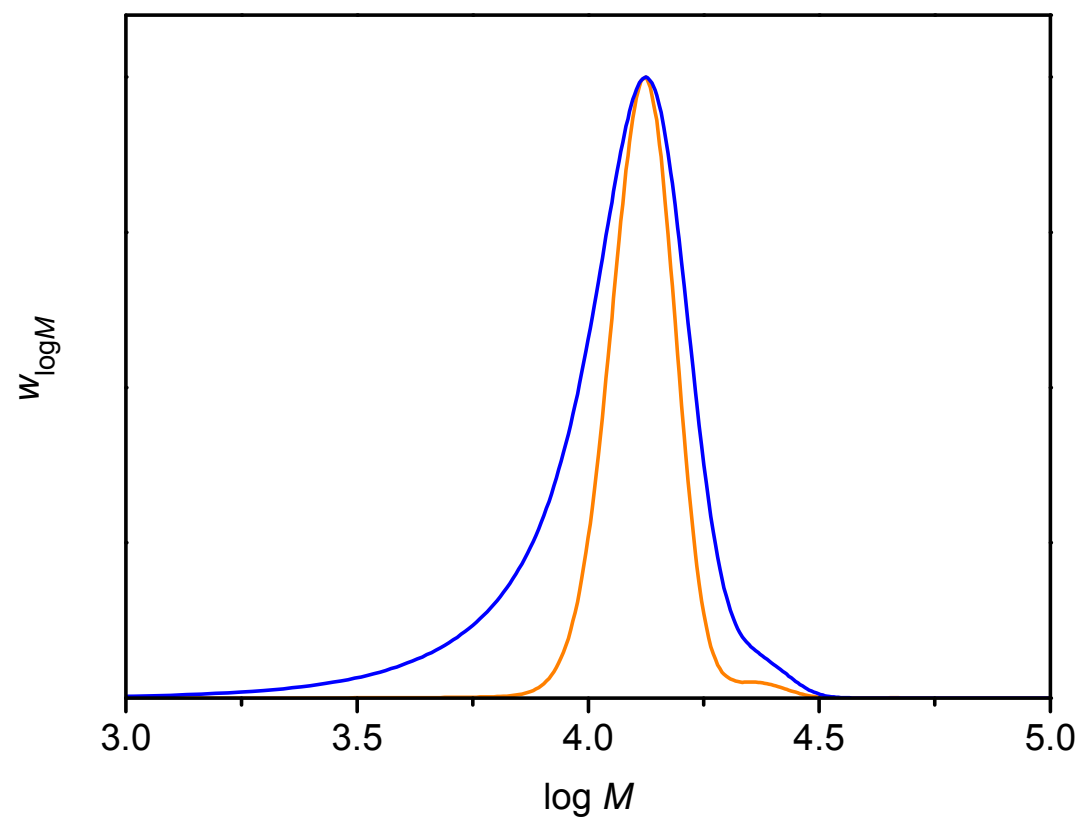

Figure 5-29. MMDs of anchored and free polymer simulated in PREDICI ${ }^{\circledR}$ with the slow fragmentation model for the surface-initiated RAFT polymerization of styrene with $k_{\mathrm{ad}, \mathrm{OL}}=0.5 \cdot k_{\mathrm{ad}} \cdot$ General reaction parameters are given in Table 5-3. 
Although the tuning of the rate coefficient $k_{\mathrm{ad}, \mathrm{OL}}$ in case of the slow fragmentation model results in a better overlap of the MMDs of the polystyrene in solution and on the surface, distinct differences to the experimental MMDs still remain visible. The low-molar-mass shoulder of the free polystyrene as well as the high-molar-mass shoulder of the grafted polystyrene is considerably less pronounced in the simulated MMDs. This may arise from the complex interplay of the rate coefficients, which were only separately altered for the simulations presented here. The number of uncertain parameters used for the simulations further only allows for the relative comparison of the MMDs and thus no conclusion can be drawn whether the slow fragmentation or the IRT model displays a more realistic model for the rate retardation observed in dithiobenzoate-mediated polymerizations. Finally, besides the achieved overlap of the MMDs, there are several other parameters that could be fitted such as the concentration of dead polymer, the number-average molar masses, and the PDI values.

\subsection{Conclusion}

The application of silica-anchored ACTA allows for the synthesis of grafted polymer via both conventional and controlled radical polymerization. The conventional radical approach yielded silica-polystyrene hybrids with a large fraction of grafted polymer, which increased towards higher total monomer conversion according to TGA, and simultaneous formation of free polystyrene probably caused by transfer reactions of anchored radicals to monomer molecules in solution. The free polymer surprisingly exhibited higher molar masses possibly due to a very low radical concentration in the interstitial solution phase. Both types of polystyrene displayed no molar-mass dependence on monomer conversion, which is expected in case of conventional radical polymerization. SEM images revealed changes in the surface topography compared to the ACTA-modified silica that create a more structured and coarse-grained surface with smaller grain sizes at higher polymer loadings. Furthermore, the silica-polymer hybrid with highest polymer loading exhibited several eye-catching spots, where parts of the polystyrene film appear to be visible at the site of cracks in the silica surface, which were observed as filaments that seem to glue the cracks together.

The additional usage of a free RAFT agent in solution induces a living character in the surface-initiated polymerization. The initiator-derived chains on the surface as well as the RAFT-derived chains in solution are polymerized in a controlled fashion with very good agreement between experimental and theoretical number-average molar masses particularly at small monomer conversions. Hence, the RAFT agent is successfully transferred to the surface 
and enables control over the molar mass of both types of polystyrenes. The anchored polystyrene exhibited slightly higher molar masses and PDI values compared with the free polystyrene. The increased PDI values were caused by a distinct high-molar-mass shoulder and broadened MMDs of the grafted polymer due to the continuous initiation process on the surface. SEC analysis further demonstrated a very low concentration of UV-active RAFT moieties in the cleaved polystyrene, which was confirmed by photographs taken before and after removal of the free polystyrene. This phenomenon could be due to the low polymer amount on the surface or side reactions that took place during the cleavage reaction with hydrofluoric acid. The achieved polymer loadings on the silica surface were quite low but not unexpected, as the chain lengths of the grafted polystyrene are approximately by a factor of 10 lower compared to the conventional radical approach. This circumstance also resulted in smaller-but still noticeable-differences of the surface topography compared to the original Si-ACTA.

The PREDICI $^{\circledR}$ simulations performed with the slow fragmentation and the IRT model enabled the identification of the high-molar-mass shoulder, which can be attributed to the termination product of a free and an anchored macroradical. Both models did not satisfactorily match the experimental MMDs of the free and anchored polystyrene using standard rate coefficients. Selected rate coefficients were altered in order to adapt the theoretical to the experimental MMDs. Especially the decrease of the addition rate coefficient for the main equilibrium between surface and solution species, $k_{\text {ad,OL }}$, allowed for improving the accordance between simulated and real MMDs via the slow fragmentation model. This could be understood in terms of a lowered addition rate caused by the hindrance in motion of tethered species and by the diffusion process of free species to the surface that is required to precede the addition reaction. The RAFT main equilibrium between two grafted species with the addition rate coefficient $k_{\mathrm{ad}, \mathrm{OO}}$ does not seem to have any impact on the degree of control of the polymerization on the surface according to the simulations. However, noticeable differences between the simulated and experimental MMDs still remain, which may be reduced by altering additional input parameters or by applying a more complex model that accounts for the heterogeneous character of the system. 



\section{PART 2:}

\section{MECHANICAL PROPERTIES OF POLYMER MATERIALS}





\section{Tensile Properties of Silica-Filled Styrene-nBA Copolymers}

This chapter highlights the tensile properties of poly(styrene-co- $n \mathrm{BA})$ filled with fumed silica particles. Copolymers doped with different amounts and types of fumed silicas were synthesized in situ by RAFT copolymerization of styrene and $n \mathrm{BA}$ with $\mathrm{AIBN}$ (see Scheme 4-1 on page 27) at $60{ }^{\circ} \mathrm{C}$ in the presence of the respective silica particles. Tensile testing is used in this work as the mechanical characterization method since it represents the most widely applied technique for measuring the mechanical properties of nanocomposites. ${ }^{[40]}$

Inorganic particles, as for example silica particles, are commonly used as fillers for polymers in order to improve their mechanical properties. ${ }^{[40,184,185]}$ Especially nanosized particles are a promising type of fillers because of their ability to enhance a polymer's mechanical behavior already at very low volume contents of 1-5\%. ${ }^{[186]}$ However, because of their high surface area, nanoparticles are prone to undergo agglomeration, which is even more favored in a hydrophobic environment due to their hydrophilic character. ${ }^{[186]}$ Such an agglomeration causes a heterogeneous dispersion of the particles in the polymer and further a worsening of the mechanical properties for most applications. Therefore, modification of the nanoparticle surface is neccessary to improve its compatibility with the polymer matrix. ${ }^{[186,187]}$ For example, nanoparticle-reinforced poly(ethene-2,6-naphthalate) exhibits higher tensile strengths and elongations at break for silica particles modified with stearic acid compared with unmodified silica particles. ${ }^{[187]}$ Hong et. al showed that maximum tensile strength and tensile modulus of reinforced poly(methyl methacrylate) were obtained by adding poly(methyl methacrylate)-modified silica nanoparticles compared with unmodified and silane-functionalized silica particles. ${ }^{[186]}$

Random copolymers of styrene and $n \mathrm{BA}$ represent an interesting class of polymers as they exhibit a broadened $T_{\mathrm{g}}$ range compared with their 
homopolymers. ${ }^{[188]}$ This, in turn, results in good damping capabilities over a wide temperature range, which is desirable for most vibration-damping applications. As shown recently, ${ }^{[188]}$ the damping level of poly(styrene-co- $n \mathrm{BA}$ ) increased upon addition of carbon black. The filling of these copolymers hence seems to be a promising way to arrive at hybrid materials with enhanced damping capabilities for specialized applications.

The surface-initiated RAFT polymerization technique, described in detail in chapter 5.2, enables the synthesis of polymer-modified silica particles that are embedded in the free matrix polymer formed in solution. Therefore, this technique was applied to produce silica-polymer hybrid materials for tensile analysis. Additionally, composites filled with two other types of silica particles, pure silica and RAFT-functionalized silica, were produced to study not only the affects of the silica content but also the affects caused by the type of silica on the tensile behavior of the resulting composites. Finally, reference copolymers with similar molar masses and monomer compositions, but without any filler, were produced to compare the mechanical properties of the composites with pure poly(styrene-co- $n \mathrm{BA}$ ).

In the first part of this chapter, the synthesis approach and the produced polymer materials are displayed. Thereafter, the results from the tensile experiments are presented and discussed in detail.

\subsection{Synthesis}

The poly(styrene-co- $n \mathrm{BA}$ )-hybrids were synthesized by addition of different types of fumed silica particles to a RAFT polymerization containing the two monomers styrene and $n \mathrm{BA}$ at equivalent amounts, toluene, RAFT agent, and AIBN (Figure 6-1) at $60{ }^{\circ} \mathrm{C}$. The three types of applied silicas were pure silica particles, ACTA-functionalized silica particles with a loading of 0.15 molecules per $\mathrm{nm}^{2}$ (Chapter 4.1), and silica that was modified with the RAFT agent propyl((trimethoxysilyl)ethylphenylmethyl)trithiocarbonate (PTPT) (Scheme 6-1) with a loading of 0.39 molecules per $\mathrm{nm}^{2}$. PTPT is tethered to the silica surface via its R-group, which enables a denser grafting of polymer chains on the surface compared to the Z-group anchorage (Chapter 3.5) in order to create a maximum copolymer loading on the silica particles. The pure silica particles were subjected to the same immobilization procedure as the functionalized silica particles (subchapter 10.2) except for the addition of the anchoring reagent. This assures identical particle conditions, as for example density and agglomeration state, for all three silica types.

The silica particles are incorporated into the copolymer during the polymerization. However, only in case of ACTA- and PTPT-modified silica there should be simultaneous formation of grafted polymer that is supposed to 
enhance the compatibility of the silica nanoparticles with the polymer matrix, which consists of the free copolymer produced in solution (Figure 6-1). For ACTA-functionalized silica, CDB (Scheme 10-1 on page 118) was used as RAFT agent in solution, as this system had proven to exhibit a proper molar-mass control (Chapter 5.2.1). In case of Si-PTPT, hexyl(phenylethyl)trithiocarbonate (HPT) (Scheme 6-1) was applied as free RAFT agent to induce molar-mass control because of its similarity to the anchored PTPT regarding the active RAFT moiety.

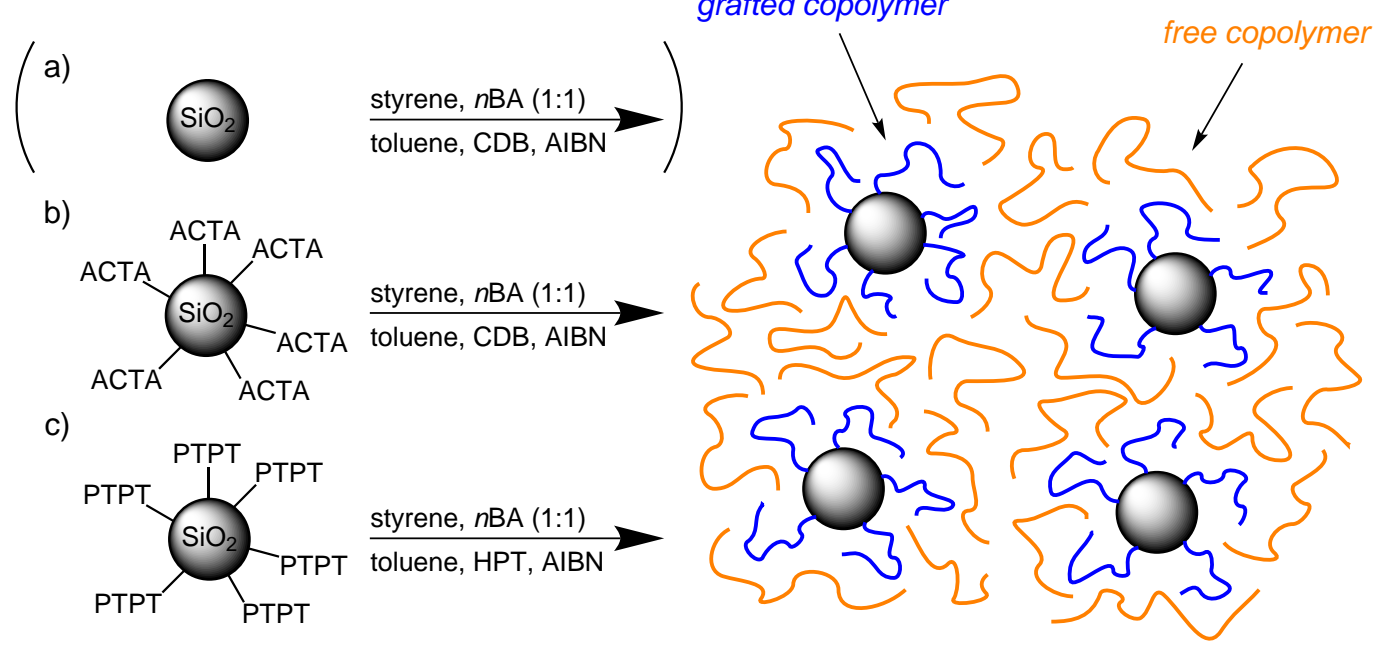

Figure 6-1. Synthesis approaches for poly(styrene-co-nBA) filled with different types of silica particles (not to scale): a) pure silica, b) ACTA-functionalized silica and c) silica functionalized with the RAFT agent PTPT. In case of pure silica, no grafted copolymer should be formed on the surface.

PTPT<smiles>CCCSC(=S)SCc1ccc(C[Si](OC)(OC)OC)cc1</smiles>

HPT<smiles>CCCCCCSC(=S)SC(C)c1ccccc1</smiles>

Scheme 6-1. RAFT agents PTPT and HPT. 
Table 6-1. Types of silica and silica weight fractions $w_{\mathrm{SiO}_{2}}$ in the styrene- $n \mathrm{BA}$ copolymers and molar styrene fraction $F_{\text {Sty }}$ and number-average molar masses $M_{\mathrm{n}}$ of the copolymers formed in solution from the RAFT copolymerization of styrene and $n \mathrm{BA}$ initiated by AIBN at $60{ }^{\circ} \mathrm{C}$.

\begin{tabular}{l|l|r|r|r}
\hline sample & silica type & $\begin{array}{c}w_{\mathrm{SiO}_{2}} \\
{[\%]}\end{array}$ & $F_{\text {Sty }}$ & $\begin{array}{c}M_{\mathrm{n}}^{\text {solution }} \\
{\left[\mathrm{g} \cdot \mathrm{mol}^{-1}\right]}\end{array}$ \\
\hline 6-1 & none & 0 & 0.61 & 33583 \\
$\mathbf{6 - 2 a}$ & $\mathrm{Si}-\mathrm{ACTA}$ & 1.5 & 0.59 & 14436 \\
$\mathbf{6 - 3 a}$ & $\mathrm{Si}-\mathrm{ACTA}$ & 2.6 & 0.59 & 29194 \\
$\mathbf{6 - 4 a}$ & $\mathrm{Si}-\mathrm{ACTA}$ & 5.2 & 0.59 & 28111 \\
$\mathbf{6 - 2 b}$ & none & 0 & 0.59 & 14951 \\
$\mathbf{6 - 3 b}$ & none & 0 & 0.59 & 29025 \\
$\mathbf{6 - 4 b}$ & none & 0 & 0.59 & 26884 \\
$\mathbf{6 - 5}$ & pure $\mathrm{SiO}_{2}$ & 2.6 & 0.59 & 27274 \\
$\mathbf{6 - 6}$ & Si-PTPT & 2.0 & 0.58 & 42671 \\
\hline
\end{tabular}

The monomeric compositions of the free matrix copolymers formed in solution were determined via Nuclear Magnetic Resonance (NMR) spectroscopy by comparing the signal between $6.1 \mathrm{ppm}$ and $8.1 \mathrm{ppm}$, which belongs to the five aromatic protons of styrene, with the signal between $3.2 \mathrm{ppm}$ and $4.3 \mathrm{ppm}$, which is caused by the two methylene protons adjacent to the ester group of $n \mathrm{BA}$. The resulting molar fractions of styrene, $F_{\text {Sty }}$, and number-average molar masses of the free polymers are listed in Table 6-1 together with the silica contents of the composites. The silica weight fraction could be adjusted by the amount of silica particles that was added to each polymerization. The molar fractions of styrene are in all cases close to 0.6, which is in very good agreement with literature values. ${ }^{[189]}$ The obtained $M_{\mathrm{n}}$ values of the polymers in solution range from about $14000 \mathrm{~g} \cdot \mathrm{mol}^{-1}$ to $43000 \mathrm{~g} \cdot \mathrm{mol}^{-1}$ due to variations in the monomer and RAFT agent concentrations as well as monomer conversions.

The unfilled copolymer 6-1 was synthesized without the presence of silica particles to produce a reference sample. The copolymer-silica composites 6-2a to 6-4a were obtained by copolymerization with ACTA-modified silica particles. In contrast to copolymer $\mathbf{6 - 1}$, the unfilled copolymers $\mathbf{6 - 2 b}$ to $\mathbf{6 - 4 b}$ were directly obtained from samples 6-2a to 6-4a by washing the latter with toluene for two days in a Soxhlet apparatus. The obtained pure copolymers 6$2 b$ to 6-4b were subsequently analyzed via NMR and SEC to check whether changes in the MMDs or monomeric compositions occurred. The results (Table 6-1) suggest no changes in molar masses and molar styrene fractions. The MMDs of the pure copolymers did not change in shape and the slight changes in the molar masses are within the uncertainty of the SEC experiment. 
Therefore, the washing process neither altered compositions nor MMDs of the copolymers according to NMR and SEC. The complete removal of the silica particles was confirmed by elemental analysis, by which no silicon was detected. Copolymer composites 6-5 and 6-6 represent the copolymer filled with pure and PTPT-modified silica particles, respectively.

\subsection{Results and Discussion}

The presentation of the results from the tensile tests is divided into two parts. In the first part, the focus is on the influence of the silica content on the tensile behavior of the composites. The second part is dedicated to the impact of the type of filler on the tensile properties by comparing styrene- $n \mathrm{BA}$ copolymers filled with differently modified silica particles. The determined tensile properties of all samples are listed in Table 6-2 and Table 6-3. It should be noted that the sample standard deviations for the determined tensile properties display large differences. Some tensile properties exhibit very small sample standard deviations, whereas in some cases the deviation even exceeds the obtained tensile property value, which can be attributed to a strong variance of the corresponding tensile tests. For this reason, the measured stress-strain curves of all specimens of a given copolymer sample are depicted in the following.

Table 6-2. Young's moduli $E$, elongations and stresses at break $\varepsilon_{\mathrm{B}}$ and $\sigma_{\mathrm{B}}$, and the corresponding sample standard deviations of pure and silica-filled copolymers of styrene and $n \mathrm{BA}$.

\begin{tabular}{l|r|r|r|r|r|r}
\hline sample & $\begin{array}{c}E \\
{[\mathrm{MPa}]}\end{array}$ & $\begin{array}{c}\Delta E \\
{[\mathrm{MPa}]}\end{array}$ & \multicolumn{1}{c|}{$\begin{array}{c}\varepsilon_{\mathrm{B}} \\
{[\%]}\end{array}$} & $\begin{array}{c}\Delta \varepsilon_{\mathrm{B}} \\
{[\%]}\end{array}$ & $\begin{array}{c}\sigma_{\mathrm{B}} \\
{[\mathrm{MPa}]}\end{array}$ & $\begin{array}{c}\Delta \sigma_{\mathrm{B}} \\
{[\mathrm{MPa}]}\end{array}$ \\
\hline 6-1 & 588 & 102 & 325.1 & 48.7 & 5.58 & 0.90 \\
6-2a & 308 & 2 & 488.2 & 21.6 & 1.52 & 0.04 \\
6-3a & 624 & 80 & 263.9 & 36.1 & 3.64 & 0.86 \\
6-4a & 596 & 2 & 213.0 & 46.4 & 3.77 & 0.05 \\
6-2b & 541 & 29 & 3.2 & 2.6 & 7.87 & 0.97 \\
6-3b & 695 & 91 & 4.7 & 1.2 & 13.2 & 2.8 \\
6-4b & 558 & 33 & 8.4 & 3.3 & 10.6 & 2.6 \\
6-5 & 644 & 33 & 48.3 & 91.3 & 9.67 & 2.19 \\
6-6 & 560 & 113 & 345.2 & 63.3 & 5.01 & 1.55 \\
\hline
\end{tabular}


Table 6-3. Strains and stresses at yield point $\varepsilon_{\mathrm{Y}}$ and $\sigma_{\mathrm{Y}}$, toughnesses $\tau$, and the corresponding sample standard deviations of pure and silica-filled copolymers of styrene and $n \mathrm{BA}$.

\begin{tabular}{l|r|r|r|r|r|r}
\hline sample & $\begin{array}{c}\varepsilon_{\mathrm{Y}} \\
{[\%]}\end{array}$ & $\begin{array}{c}\Delta \varepsilon_{\mathrm{Y}} \\
{[\%]}\end{array}$ & $\begin{array}{c}\sigma_{\mathrm{Y}} \\
{[\mathrm{MPa}]}\end{array}$ & $\begin{array}{c}\Delta \sigma_{\mathrm{Y}} \\
{[\mathrm{MPa}]}\end{array}$ & $\begin{array}{c}\tau \\
{\left[\mathrm{MJ} \cdot \mathrm{m}^{-3}\right]}\end{array}$ & $\begin{array}{c}\Delta \tau \\
{\left[\mathrm{MJ} \cdot \mathrm{m}^{-3}\right]}\end{array}$ \\
\hline 6-1 & 4.2 & 0.1 & 14.2 & 1.9 & 21 & 3 \\
6-2a & 4.8 & 0.7 & 4.53 & 0.14 & 12 & 1 \\
6-3a & 4.2 & 0.0 & 12.2 & 1.9 & 17 & 2 \\
6-4a & 4.3 & 0.0 & 12.2 & 1.6 & 14 & 2 \\
6-2b & - & - & - & - & 0.20 & 0.21 \\
6-3b & - & - & - & - & 0.49 & 0.16 \\
6-4b & - & - & - & - & 0.77 & 0.28 \\
6-5 & 4.3 & 0.1 & 13.0 & 0.40 & 3.6 & 6.4 \\
6-6 & 4.7 & 0.8 & 10.7 & 1.9 & 25 & 7 \\
\hline
\end{tabular}

\subsubsection{Impact of Silica Content}

The seven samples 6-1 to 6-4b are examined for studying the influence of the filler content on the tensile performance of the silica-copolymer hybrids. The three composites (6-2a to 6-4a) allow for comparing silica-filled copolymers with different silica fractions, whereas the extracted pure copolymers $\mathbf{6 - 2 b}$ to 6$4 \mathrm{~b}$ enable the comparison between the filled und unfilled material of exactly the same styrene $-n \mathrm{BA}$ copolymer. This procedure allows for the direct comparison of the same polymer with and without filler to eliminate other sources of impact on the tensile behavior such as the MMD of the copolymers. The pure poly(styrene-co- $n \mathrm{BA}$ ) 6-1 acts as additional reference in form of the unfilled copolymer. Its stress-strain curves (Figure 6-2) display a very pronounced plastic region beyond the yield point, in which the apparent stresses decrease first and increase again at higher elongations up to the break point.

Samples 6-2a to 6-4a display significant differences in their tensile behavior compared with the pure copolymer 6-1. The stress-strain curves of the styrene- $n$ BA copolymer with the lowest silica content of $1.5 \mathrm{wt} .-\%, 6-2 \mathrm{a}$ (blue curves in Figure 6-3), feature lower overall stresses but at the same time higher elongations at break. In turn, the composites 6-3a and 6-4a with higher silica filler amounts of $2.6 \%$ and $5.2 \%$ (blue curves in Figure 6-4 and Figure 6-5) display smaller elongations at break compared with copolymer 6-1, but increased overall stresses compared with sample 6-2a. However, the copolymer of sample 6-2a has a significantly smaller number-average molar mass than the copolymers of 6-1, 6-3a and 6-4a (Table 6-1). Therefore, the smaller chain 
length of 6-2a may also largely affect its tensile performance, which hampers the comparison with composites 6-1, 6-3a and 6-4a. This is also suggested by the determined tensile properties for sample 6-2a (Table 6-2 and Table 6-3), which do not seem to fit into the remaining values. Specifically the Young's modulus, stress and strain at yield point, and the toughness are considerably smaller compared with the values obtained from the other composites. It should be pointed out, though, that in many cases a maximum elongation at break is observed in silica-polymer nanocomposites at lower filler amounts, ${ }^{[40]}$ hence the silica content of $1.5 \mathrm{wt} . \%$ for sample 6-2a may nonetheless be responsible for the larger $\varepsilon_{\mathrm{B}}$ value compared with the pure copolymer 6-1. However, for the reasons mentioned above, the study of correlations between the silica content and the tensile properties will be limited to samples 6-1, 6-3a and 6-4a, whose copolymers have similar molar masses.

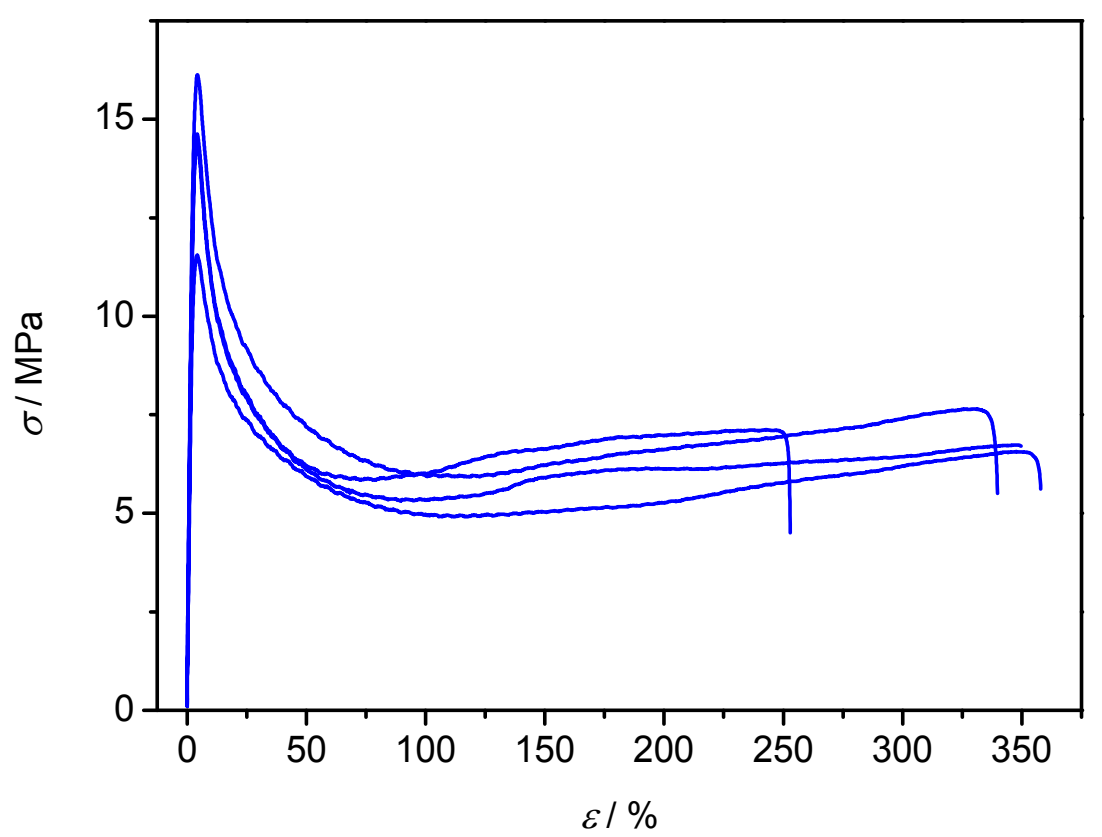

Figure 6-2. Stress-strain curves of four specimens of pure styrene- $n$ BA copolymer 6-1. 


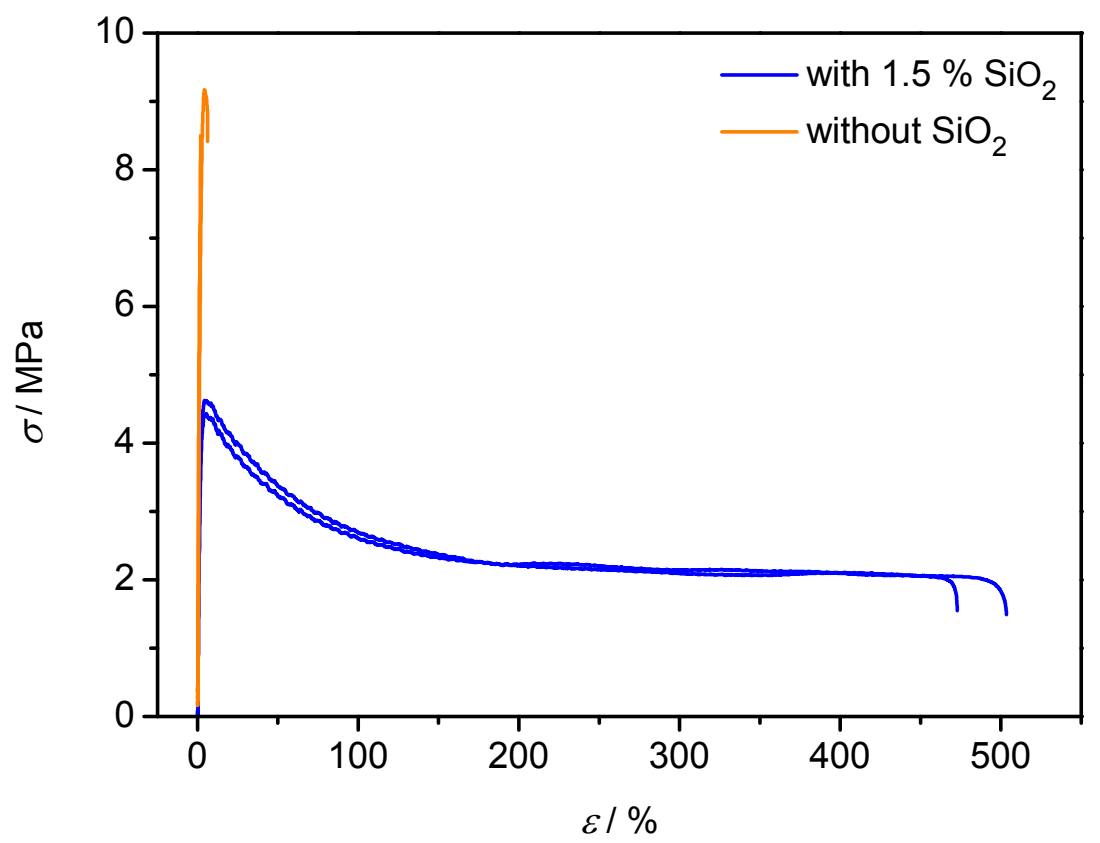

Figure 6-3. Stress-strain curves of two specimens of composite 6-2a filled with Si-ACTA and of three specimens of the extracted pure copolymer 6-2b. See Table 6-2 and Table 6-3 for tensile properties.

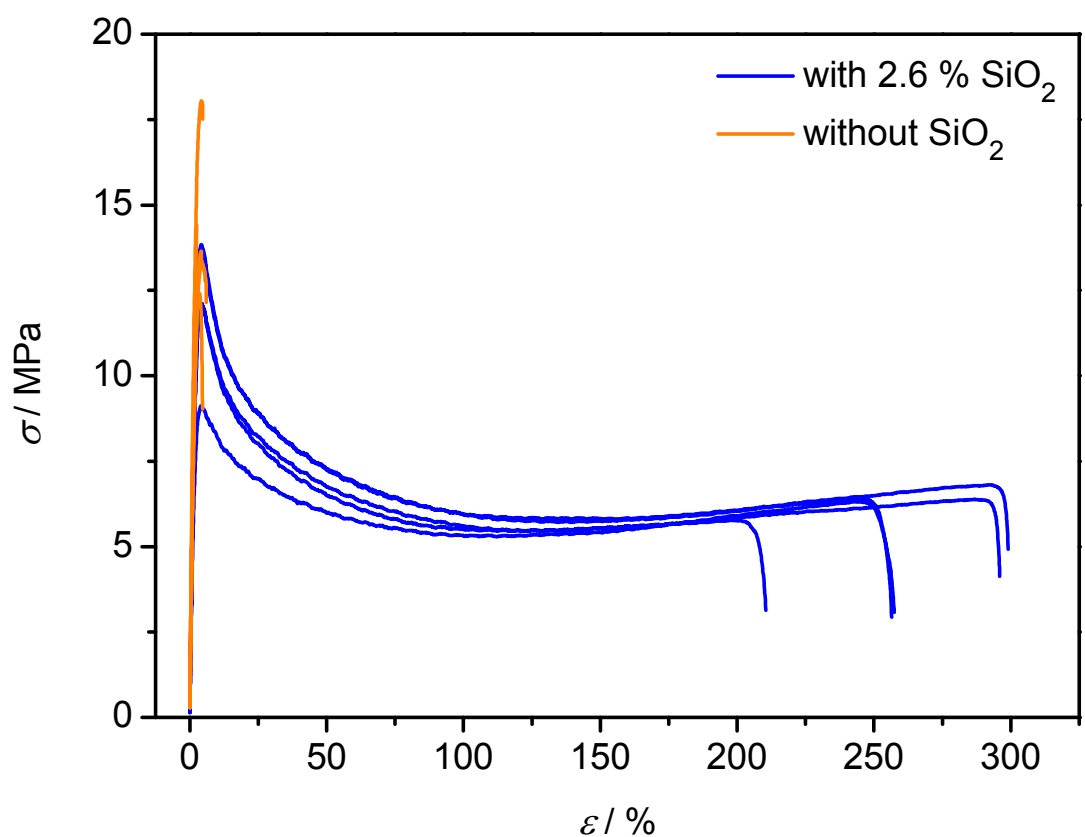

Figure 6-4. Stress-strain curves of five specimens of composite 6-3a filled with Si-ACTA and of six specimens of the extracted pure copolymer 6-3b. See Table 6-2 and Table 6-3 for tensile properties. 


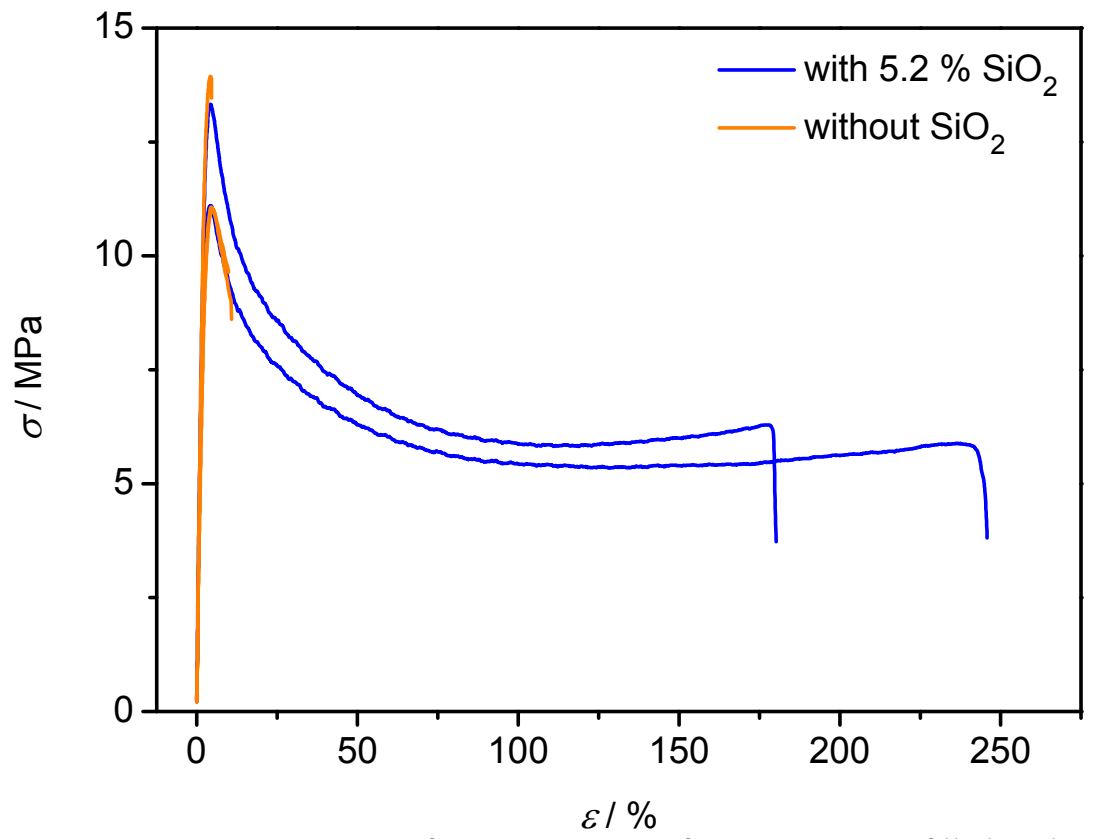

Figure 6-5. Stress-strain curves of two specimens of composite 6-4a filled with Si-ACTA and of three specimens of the extracted pure copolymer 6-4b. See Table 6-2 and Table 6-3 for tensile properties.

As can be seen in Figure 6-6, the elongation at break and the toughness of the silica-copolymer hybrids both decrease with increasing silica content. Especially the diminished $\varepsilon_{\mathrm{B}}$ values at higher silica contents are in line with literature findings. ${ }^{[187]}$ Interestingly, the Young's moduli of the styrene $-n \mathrm{BA}$ copolymers 6-3a and 6-4a are not significantly affected by the silica filler (Table 6-2), although pure silica features an elastic modulus of about $70 \mathrm{GPa}$ and thus usually leads to an increase of $E$ when used as a filler. ${ }^{[184]}$ However, constant Young's moduli have also been observed by Kim et al. for silica-filled poly(ethene-2,6-naphthalate) upon the addition of silica particles that were coated with multilayers of stearic acid. ${ }^{[187]}$ Other tensile properties that are smaller compared with the pure copolymer 6-1-but do not correlate with the silica content-are the stresses at yield and break point (Table 6-2 and Table 6-3).

The obtained stress-strain curves of the pure copolymers $6-2 \mathbf{b}$ to $\mathbf{6 - 4 b}$ (orange lines in Figure 6-3 to Figure 6-5), which were extracted from the composites 6-2a to 6-4a, provide quite remarkable results. After removal of the silica filler, all three copolymers exhibit a pronounced brittle behavior, indicated by the considerable reduction of elongations at break. As a consequence, the toughnesses are diminished as well and the corresponding stresses at break are much higher, since the majority of samples break close to the linear regime and thus display no yield point (Table 6-2 and Table 6-3). 
These results are noteworthy, as they are contradictory to the results obtained from tensile testing of samples 6-1 and 6-2a to 6-4a that indicate rather a reduction than an increase of $\varepsilon_{\mathrm{B}}, \sigma_{\mathrm{B}}$, and $\tau$ induced by the silica filler. Even though the reference copolymer 6-1 has a molar mass that is higher compared with copolymers $6-2 b$ to $6-4 b$, the difference appears to be too small to create a more ductile behavior due to increased entanglements. The same assumption probably holds for the influence of the initiator-derived chains grafted on the silica particles, which are removed along with the silica in the washing procedure. The amount of this material is rather small, as shown in subchapters 5.2.2 and 5.2.4, and hence its absence probably does not affect the tensile properties to such extent.

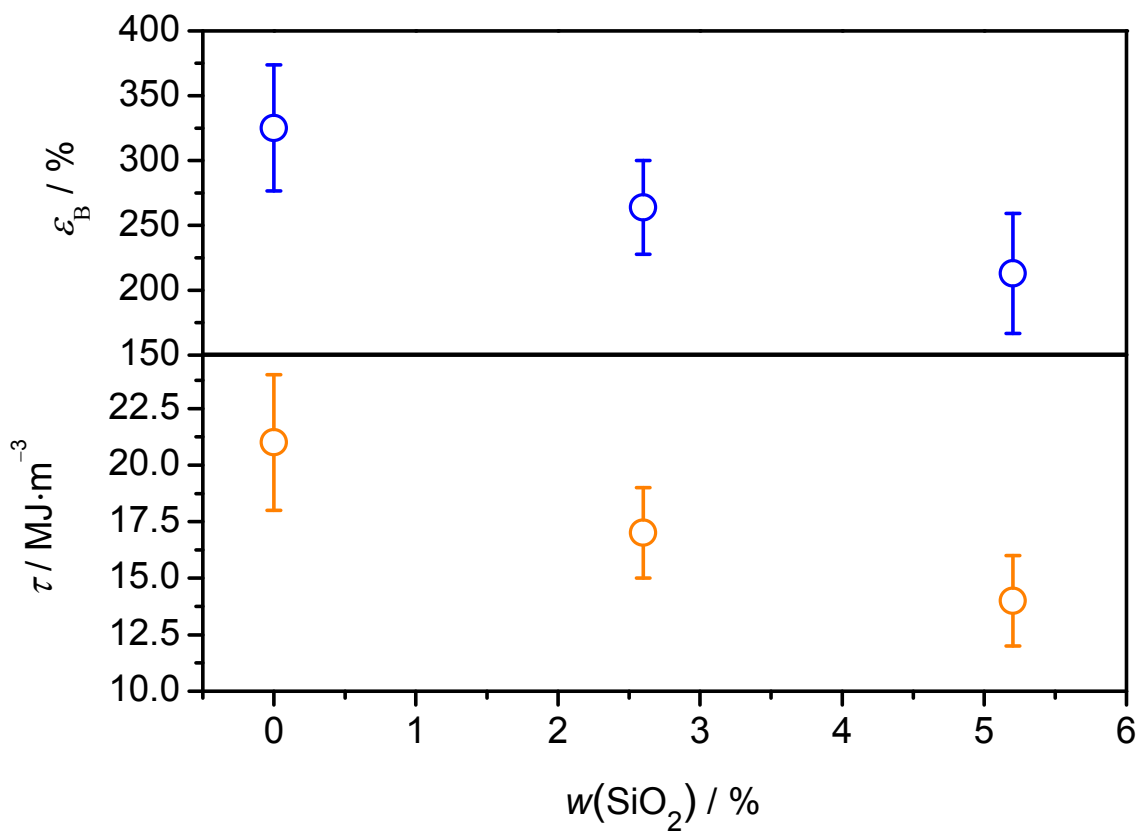

Figure 6-6. Elongations at break and toughnesses obtained from stress-strain analysis of silicacopolymer hybrids 6-1, 6-3a, and 6-4a filled with different silica contents.

Although the origins of these changes in tensile behavior are unclear at this stage, it should be stressed that these results were obtained from measurements of numerous specimens from each material, while at the same time assuring identical copolymer compositions and molar masses, determined by NMR and SEC, respectively. Therefore, the detected change from ductile behavior of the filled styrene- $n$ BA copolymers 6-2a to 6-4a to brittle behavior of the unfilled copolymers $\mathbf{6 - 2 b}$ to $\mathbf{6}-\mathbf{4 b}$ does not seem to be a matter of coincidence. One feasible explanation may be a change of the copolymer structure induced by the 
washing process, which is performed with toluene as the solvent at elevated temperatures. Cross-linking reactions, for instance, may alter the copolymer largely enough to cause the drastic changes in the tensile properties. Although NMR and SEC analysis did not indicate any change in the copolymer structure, the extent of such changes may be too small in order to be detected. In contrast to the $\varepsilon_{\mathrm{B}}, \sigma_{\mathrm{B}}$, and $\tau$ values, the tensile moduli $E$ of copolymers $\mathbf{6 - 2 b}$ to $\mathbf{6}-\mathbf{4 b}$, as for samples 6-1 and 6-2a to 6-4a, do not feature significant differences (Table 6-2).

\subsubsection{Impact of Silica Type}

The influence of the silica filler type on the tensile properties is probed by the comparison of three different composites with similar silica contents that were synthesized in the presence of pure silica (sample 6-5), ACTA-functionalized silica (sample 6-3a) and PTPT-modified silica (sample 6-6), respectively.

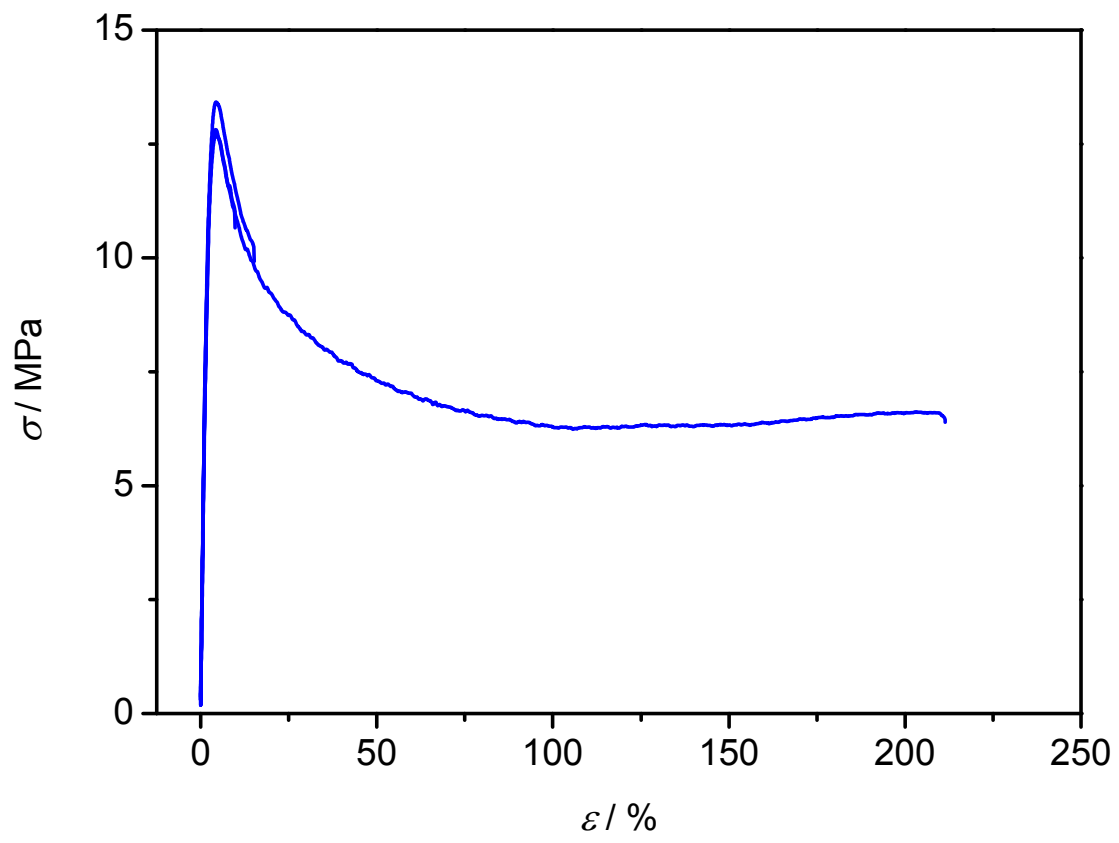

Figure 6-7. Stress-strain curves of five specimens of composite 6-5 filled with pure silica (curves largely overlap). See Table 6-2 and Table 6-3 for tensile properties.

The stress-strain diagrams for the styrene- $n \mathrm{BA}$ copolymer filled with nonfunctionalized silica particles 6-5 (Figure 6-7) show that all but one specimen break in proximity to the yield point. In contrast to that, the copolymer 6-6 
filled with PTPT-modified silica exhibits significantly higher elongations at break (Figure 6-8). For both samples, neither the Young's modulus nor the yield point data display any significant deviations from sample 6-3a (Table 6-2 and Table 6-3). However, distinct differences for the break points as well as the toughnesses of the three composites can be detected. For composite 6-5 with pure silica filler the break points are given by high stresses and low strains, whereas in case of modified silica particles the composites exhibit noticeably higher strains and lower stresses at break (Figure 6-9). Furthermore, the toughnesses are significantly higher for the functionalized silica fillers with a maximum value for the RAFT-modified silica particles (Figure 6-10).

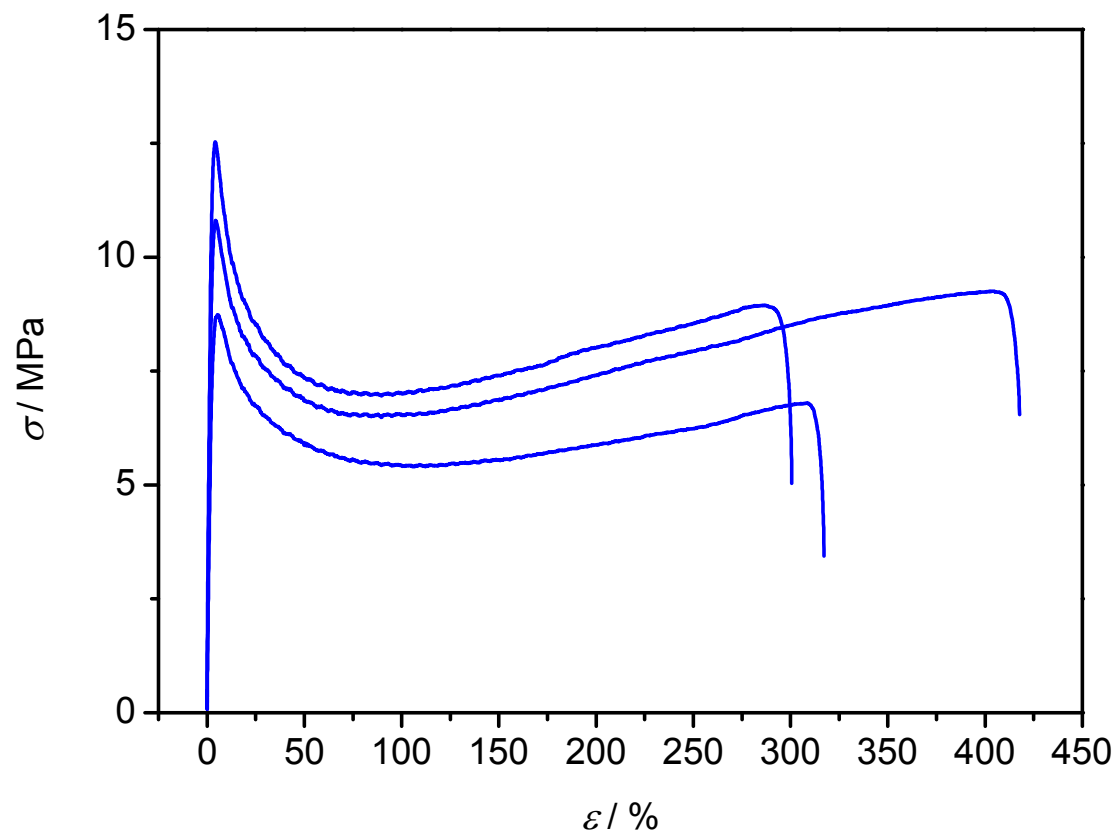

Figure 6-8. Stress-strain curves of three specimens from composite 6-6 filled with silica type SiPTPT. See Table 6-2 and Table 6-3 for tensile properties. 


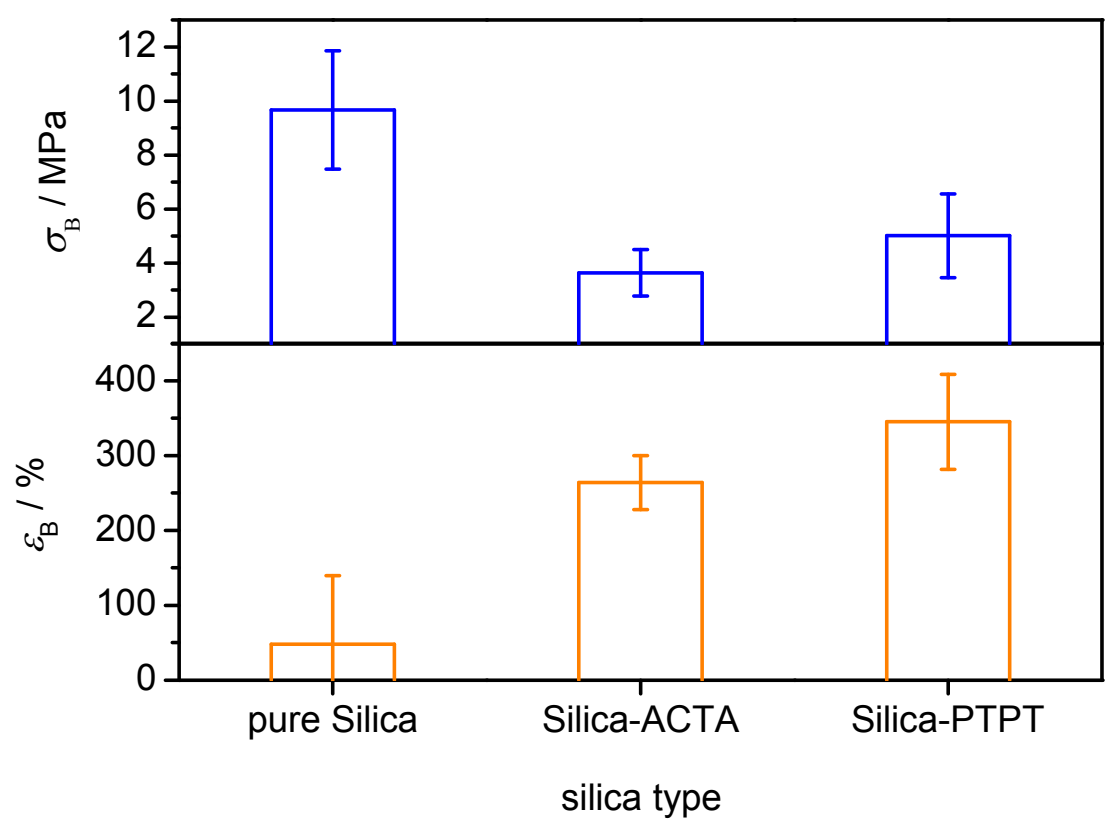

Figure 6-9. Stresses and elongations at break obtained from stress-strain analysis of silicacopolymer hybrids 6-3a, 6-5, and 6-6 filled with different types of silica.

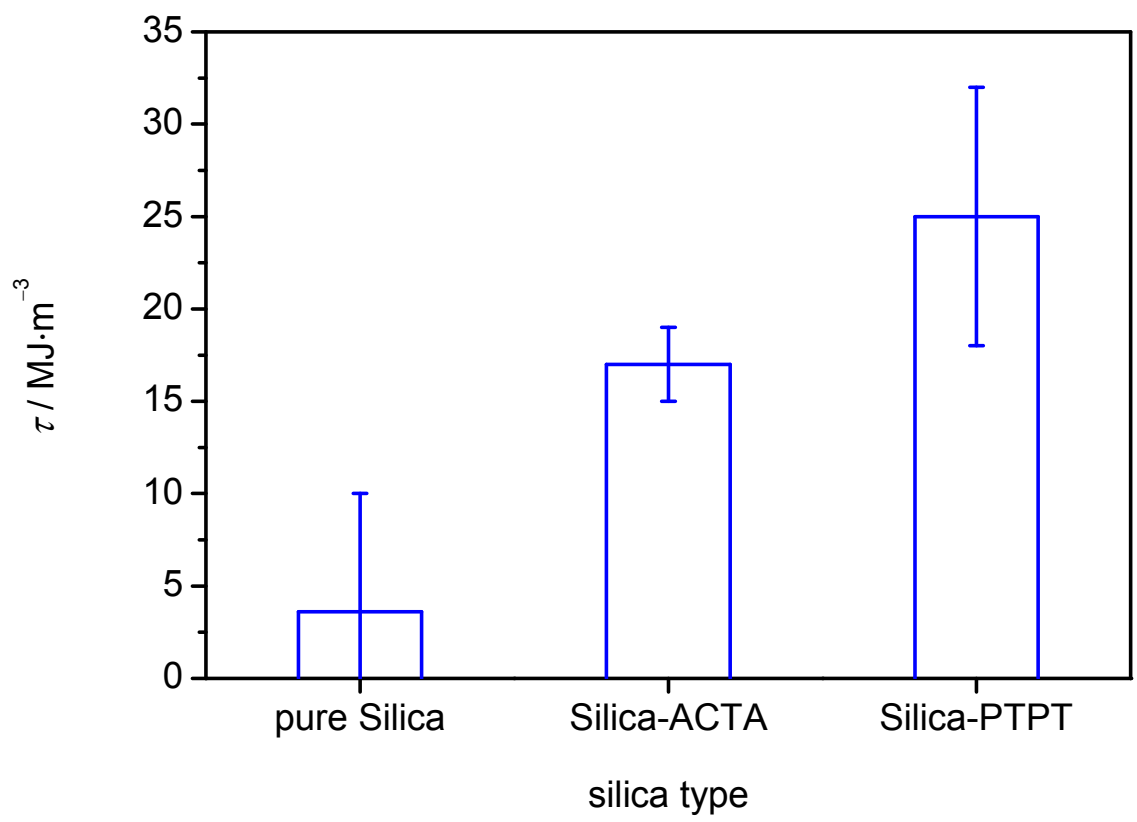

Figure 6-10. Toughnesses obtained from stress-strain analysis of silica-copolymer hybrids 6-3a, 6-5, and 6-6 filled with different types of silica. 
A crucial property of the silica-copolymer composites is the interaction between the filler and the matrix copolymer. This interaction is largely governed by the amount of grafted copolymer chains on the surface of the silica particles, as these chains enable the compatibility between the silica filler and the matrix copolymer. The copolymer loadings on the particles were determined for all three types of silica by TGA after removal of the free matrix copolymer (Figure 6-11).

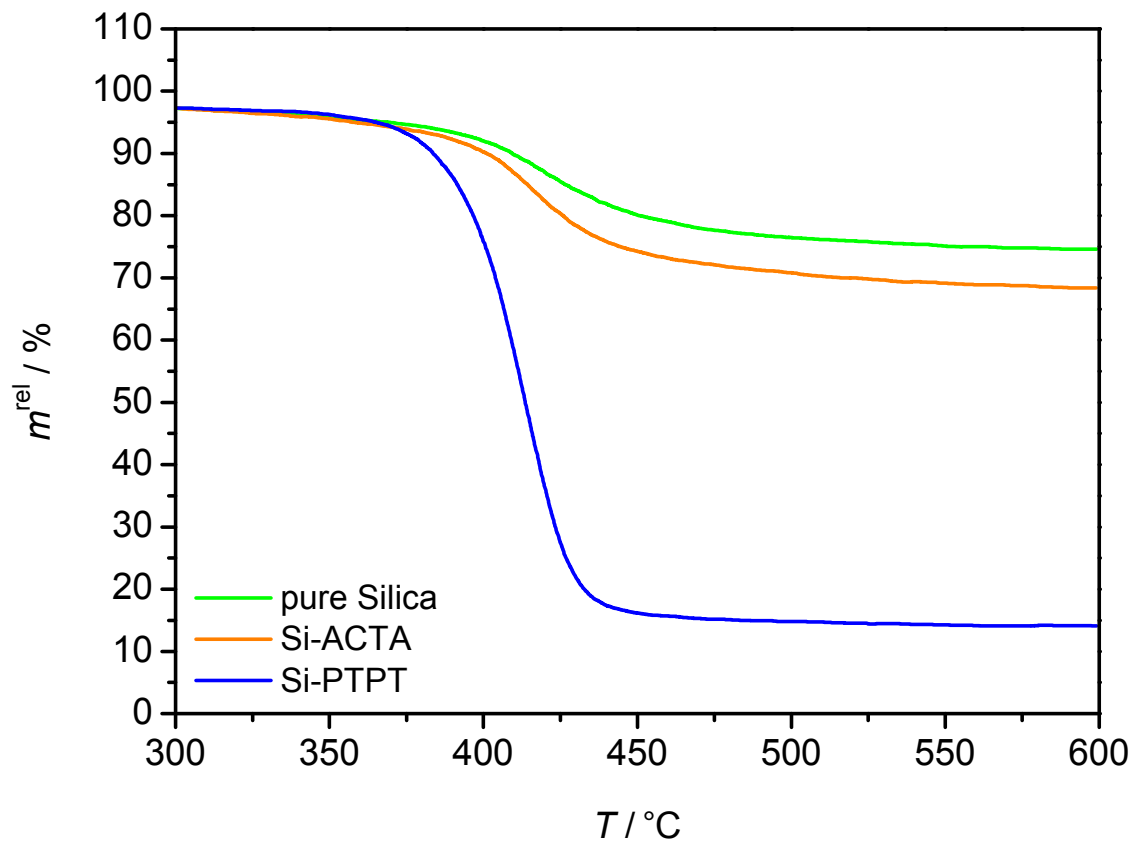

Figure 6-11. TGA curves from $300{ }^{\circ} \mathrm{C}$ to $600{ }^{\circ} \mathrm{C}$ of pure silica particles and of silica particles functionalized with ACTA and PTPT, respectively, after RAFT copolymerization of styrene and $n \mathrm{BA}$ and removal of the free matrix copolymer.

Surprisingly, the TGA curve of pure silica indicates the presence of about $23 \%$ copolymer on the surface despite the absence of a grafted reagent on the particle surface. The origin of this finding is not certain at this stage, but could possibly be ascribed to a physical adsorption of the free copolymer chains on the silica surface due to the high polarity of the $n \mathrm{BA}$ units. The entrapment of free copolymer chains in between the silica particles appears rather unlikely, as the particles were repeatedly washed. Therefore, copolymer that is attached to the surface by an interaction between the $\mathrm{OH}$-groups on the silica surface and the copolymer chains displays the most likely assumption. The ACTA-modified particles contained approximately $29 \%$ grafted copolymer after polymerization, whereas in the case of PTPT-functionalized silica particles a copolymer loading 
on the silica surface of about $83 \%$ was achieved. This high value for the polymer loading is probably caused by the higher grafting density of RAFT agent compared with the loading of ACTA on the silica surface and further by the simultaneous start of chain growth for the anchored radicals according to the RAFT mechanism. Consequently, the copolymer composite filled with PTPT-modified silica particles also features the highest interaction between the copolymer matrix and its filler that further results in the highest average elongation at break and average toughness compared with the other two composites, as depicted in Figure 6-9 and Figure 6-10. Both $\varepsilon_{\mathrm{B}}$ and $\tau$ increase in the order of pure, ACTA-modified and PTPT-modified silica particles used as fillers, which is in alignment with the fraction of surface-grafted copolymer and thus also with the interaction between the filler and the matrix copolymer. Interestingly, $\varepsilon_{\mathrm{B}}$ and $\tau$ differ largely for pure and ACTA-modified silica despite the rather small differences of the copolymer loadings on the silica surface according to TGA. This finding could be attributed to the different type of interaction to the surface, which consists of covalent bonds in case of ACTAmodified silica and-presented as the most feasible suggestion here-of intermolecular interactions in case of pure silica.

\subsection{Conclusion}

In this chapter, a routine has been presented that allows for synthesizing silicafilled styrene- $n \mathrm{BA}$ copolymers in situ by RAFT polymerization in the presence of fumed silica particles. The silica particles were modified with anchored initiator and RAFT agent, respectively, to enable a grafting of copolymer on the surface during polymerization that results in a higher interaction between the free copolymer matrix and its filler.

The addition of $1.5 \mathrm{wt} .-\%$ of ACTA-functionalized silica particles lead to an increase of the elongation at break compared with the neat copolymer without filler, whereas larger amounts of silica (2.6 wt.-\% and $5.2 \mathrm{wt.} . \%)$ resulted in a decrease of both the elongation at break and the toughness of the copolymer hybrid materials compared with the pure reference copolymer.

Selected copolymer composites were washed with toluene to separate the pure copolymer from the silica filler. This approach should enable the direct comparison of identical copolymers with and without silica filler, affirmed by NMR, SEC, and elemental analysis, to eliminate other sources of impact such as the molar mass of the polymers. The results of these tensile tests were quite remarkable, as the silica-filled samples of poly(styrene-co- $n \mathrm{BA})$ displayed a ductile behavior, while the corresponding silica-free copolymers exhibited a pronounced brittle behavior, which was evident from low $\varepsilon_{\mathrm{B}}$ and high $\sigma_{\mathrm{B}}$ values determined by stress-strain analysis. The reasons for the drastic changes 
in the tensile behavior remain unclear at the moment, but could possibly be assigned to side-reactions, as for instance cross-linking reactions, which may occur during the washing process.

Finally, three composites of poly(styrene-co- $n \mathrm{BA}$ ) filled with similar amounts of differently treated silica particles were prepared to study the influence of the type of silica filler on the tensile performance. The three types of silica were pure, ACTA-functionalized, and PTPT-modified fumed silica. The amount of copolymer on the surface of the filler after copolymerization increases in the same order, which was affirmed by TGA. Although the pure silica particles did not contain any grafted reagent on the surface, TGA indicated the presence of attached copolymer that is suggested to arise from physisorption of polar $n \mathrm{BA}$ monomer units on the silica surface. The amount of anchored copolymer was particularly high in case of immobilized RAFT agent PTPT with a copolymer loading of $83 \%$ on the silica particles, since the surface-initiation starts almost simultaneous for all chains on the surface compared with grafted ACTA that dissociates continuously. The tensile testing of styrene- $n \mathrm{BA}$ copolymers filled with pure silica particles revealed low elongations and high stresses at break as well as low toughnesses of the composites. The low fraction of attached copolymer in case of pure silica particles, most probably caused by physisorption, appears not to enable an effective compatibility of the hydrophilic silica particles with the hydrophobic copolymer. Additionally, copolymer chains that are merely physisorbed on the surface are most probably not able to withstand the high stresses which occur during tensile testing and hence the overall tensile properties are diminished. In contrast to that, the silicas modified with ACTA and PTPT, respectively, yield composites with significantly enhanced tensile performances, especially regarding the elongations at break and the toughnesses. This finding was particularly pronounced in case of PTPT-modified silica particles, as they feature a much larger amount of copolymer on the surface. This, in turn, provides an enhanced interaction between the matrix copolymer and its silica filler that results in improved tensile properties. 


\section{Relaxation Processes of ABA Type $t \mathrm{BA}-\mathrm{AA}$ Copolymers}

In this chapter relaxation modes of tailored ABA type $t \mathrm{BA}-\mathrm{AA}$ copolymers are covered in detail. ${ }^{[190]}$ Specifically, a novel secondary relaxation process, which emerges from the controlled insertion of AA into the outer chain parts of poly $(t \mathrm{BA})$, as well as the $\alpha$-transition (glass transition) and a high-temperature relaxation are examined. The copolymers were synthesized via RAFT polymerization because of its robustness, versatility, and straightforwardness. Relaxation modes were determined by means of DMA, as it is a very well suited technique for determining molecular relaxations in polymer materials. ${ }^{[4]}$

Very recently, Omayu and co-workers showed that the introduction of intermolecular hydrogen bonds into copolymers of $\mathrm{N}$-substituted maleimides and isobutene significantly affects the relaxation spectra. ${ }^{[11]}$ In particular, the $\beta$-relaxation, which is generally referred to as a side-chain movement (although its mechanism is not yet clearly understood), shifted in temperature as carboxy side groups were inserted. Hydrogen bonds can even lead to the occurance of an additional relaxation mode, as it is the case in copolymers of ethene and MAA with a non-random distribution of the two comonomers. ${ }^{[43]}$ The observed relaxation mode, which was localized in the temperature range between the $\gamma$ relaxation and the glass transition, was discussed as a flipping of short polyethene segments, which were fixed between segments containing MAA units that formed hydrogen bonds. For this situation the term "chemical confinement" (cc) was applied, since the MAA confined the motion of the polyethylene segments. However, it is still unknown if the assumption of the chemical confinement is a general concept.

One simple model to follow up this hypothesis is to check the relaxation modes of tailored ABA type copolymers, in which only the outer A-blocks are able to form hydrogen bonds and thus create a chemical confinement, that is the inner B-block is freely moving between the fixed outer A-blocks. To achieve such a situation, two copolymerizable monomers are required, of which only 
one is capable of forming hydrogen bonds and is thus inserted in the outer Ablocks. Two monomers that meet these requirements and are therefore used in this work are $t \mathrm{BA}$ and $\mathrm{AA}$ (Scheme 7-1). Both monomers can be easily polymerized in a controlled fashion via the RAFT technique and additionally allow a simple copolymerization due to their structural similarity.

At the beginning of this chapter, the two-step synthesis is introduced along with the resulting ABA type $t \mathrm{BA}-\mathrm{AA}$ copolymers. Subsequently, the results from the DMA measurements, specifically relaxation spectra and the corresponding progress of the relative sample heights, are presented and discussed in detail. ${ }^{[144]}$

\subsection{Synthesis}

In order to achieve the desired ABA type copolymer structure, the bifunctional symmetric trithiocarbonate dimethyl-2,6-di(propyltrithiocarbonyl)heptanedioate (DPTH) was used as the RAFT agent, depicted in Scheme 7-1. This RAFT agent allows for the straightforward synthesis of ABA block copolymers, as the product of the first polymerization (inner B-block, poly $(t \mathrm{BA})$ ) can be used as a macromolecular RAFT agent to add the outer A-blocks via an extension polymerization in the presence of a second monomer (Scheme 7-2). However, in this work the macromolecular RAFT agent was not copolymerized with a pure comonomer but rather with a mixture of $t \mathrm{BA}$ and $\mathrm{AA}$ at a molar ratio of 11:1. Hence these polymers resemble a structure, in which AA units are incorporated into the outer parts of poly $(t \mathrm{BA})$ chains in a controlled fashion. Although this constitution is quite different from the one of a true block copolymer, the terms A- and B-blocks are used for the different segments in order to distinguish and emphasize the interpretations of the observed relaxations.

tBA<smiles>C=CC(=O)OC(C)(C)C</smiles>

AA<smiles>C=CC(=O)O</smiles>

DPTH<smiles>CCCSC(=S)SC(CCCC(SC(=S)SCCC)C(=O)OC)C(=O)OC</smiles>

Scheme 7-1. Monomers $t$ BA and AA as well as the RAFT agent DPTH used for the synthesis of the ABA type copolymers. 
The evolution of molar masses and PDI values for the synthesis of the B-blocks, depicted in Figure 7-1, indicates a fully effective RAFT polymerization with increasing molar masses and low PDI values towards higher monomer conversion. The experimental values are in relatively good agreement with the theoretical values, which were calculated from equation 3-16. Therefore, the RAFT agent DPTH appears to be well-suited for the controlled synthesis of the copolymers.

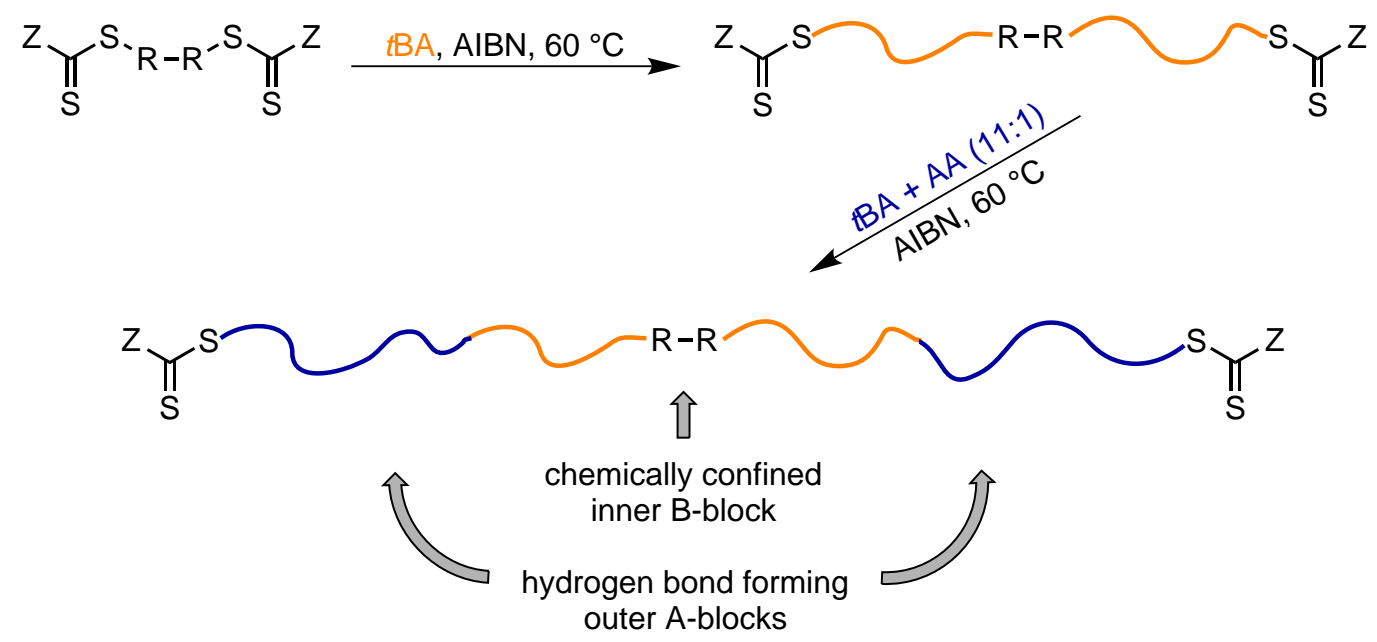

Scheme 7-2. Synthesis approach for producing $t \mathrm{BA}-\mathrm{AA}$ copolymers, in which only the outer Ablocks contain AA and can thus form hydrogen bonds that chemically confine the inner Bblock.

A relevant issue for the second reaction step, which is the extension polymerization of the macromolecular RAFT agents to yield the ABA type copolymers, is the actual incorporation of AA into the outer A-blocks. To ensure the regular insertion of AA in the course of copolymerization, the molar ratios of $t \mathrm{BA}$ and $\mathrm{AA}$ in the polymerization solution were determined during a copolymerization of the two monomers via ${ }^{1} \mathrm{H}-\mathrm{NMR}$. For this purpose, the integral peak intensities that originate from the olefinic protons, specifically the signals of the two monomers between $5.50 \mathrm{ppm}$ and $5.75 \mathrm{ppm}$ and between $6.05 \mathrm{ppm}$ and $6.30 \mathrm{ppm}$, were used to obtain the molar monomeric ratios. As shown in Figure 7-2, the molar monomeric ratio of $t \mathrm{BA}$ and AA changes from about 5.6 before copolymerization to about 4.0 at $90 \%$ conversion. Due to the small decrease of the molar $t \mathrm{BA}$ fraction during copolymerization, this comonomer is slightly more present in the resulting A-blocks of the copolymer compared with the starting ratio, although the difference is negligible. Hence 
the comonomer AA is incorporated steadily into the outer A-blocks close to the adjusted starting ratio of the two comonomers.

The final ABA type $t \mathrm{BA}-\mathrm{AA}$ copolymers that are subjected to DMA are listed in Table 7-1 together with the $M_{\mathrm{n}}$ values of the B- and A-blocks as well as the PDI values of the entire copolymers. The PDI values are in the range of 1.19 to 1.34 and thus well below the limit of 1.5 for conventional radical polymerizations, which indicates a good polymerization control. The molar masses of the inner B-blocks were adjusted via RAFT polymerization to be in the range of approximately $1000 \mathrm{~g} \cdot \mathrm{mol}^{-1}$ to $10000 \mathrm{~g} \cdot \mathrm{mol}^{-1}$, while the molar masses of the A-blocks were set to be in the range of about $8000 \mathrm{~g} \cdot \mathrm{mol}^{-1}$ to $14000 \mathrm{~g} \cdot \mathrm{mol}^{-1}$. This setup not only allows for studying relaxations that emerge from short chain segments, namely the B-blocks, but it also permits the analysis of possible dependencies of the temperatures, at which the relaxations and the changes in relative sample height occur, on the chain lengths of the A- and Bblocks. The corresponding results, determined via DMA, are subject of the following subchapter.

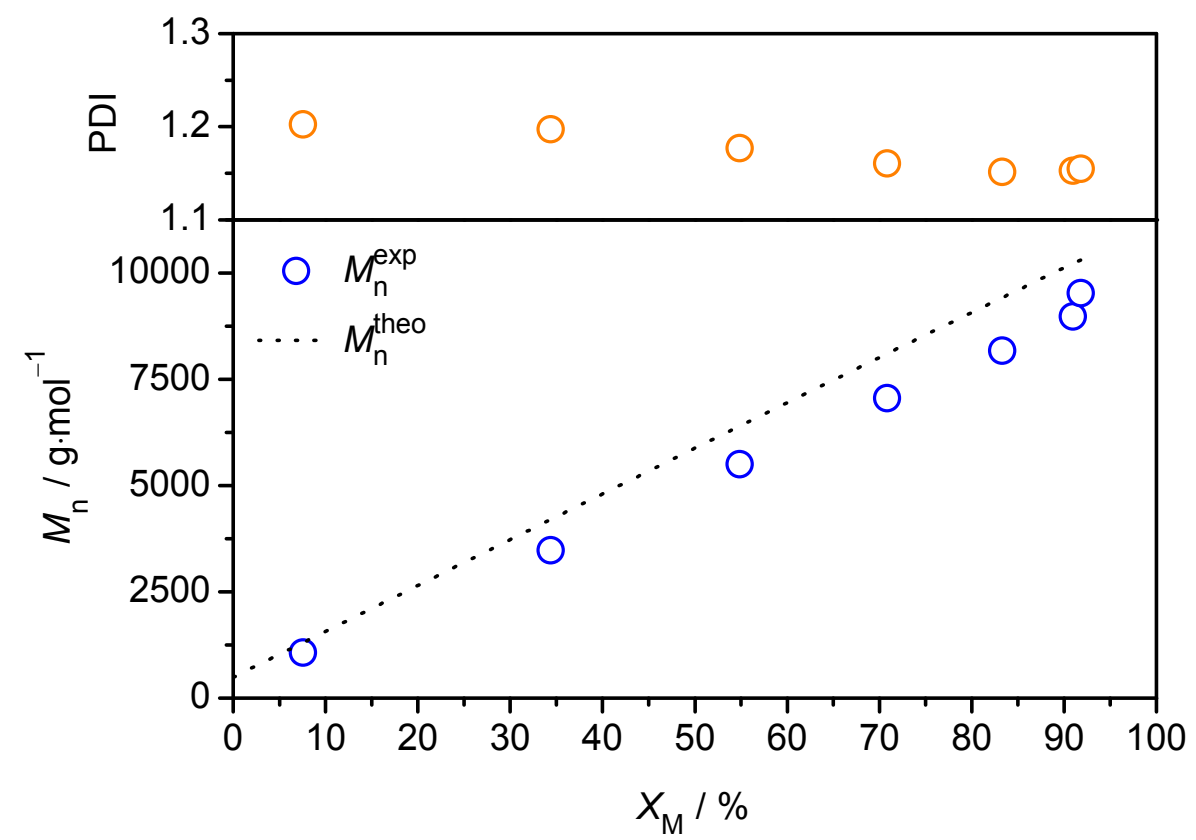

Figure 7-1. Evolution of experimental and theoretical number-average molar masses $M_{\mathrm{n}}$ and PDI with total monomer conversion $X_{M}$ for the RAFT polymerization of $t \mathrm{BA}\left(3.4 \mathrm{~mol} \cdot \mathrm{L}^{-1}\right)$ in toluene mediated by DPTH $\left(40 \mathrm{mmol} \cdot \mathrm{L}^{-1}\right)$ and initiated by AIBN $\left(4.8 \mathrm{mmol} \cdot \mathrm{L}^{-1}\right)$ at $60{ }^{\circ} \mathrm{C}$. 


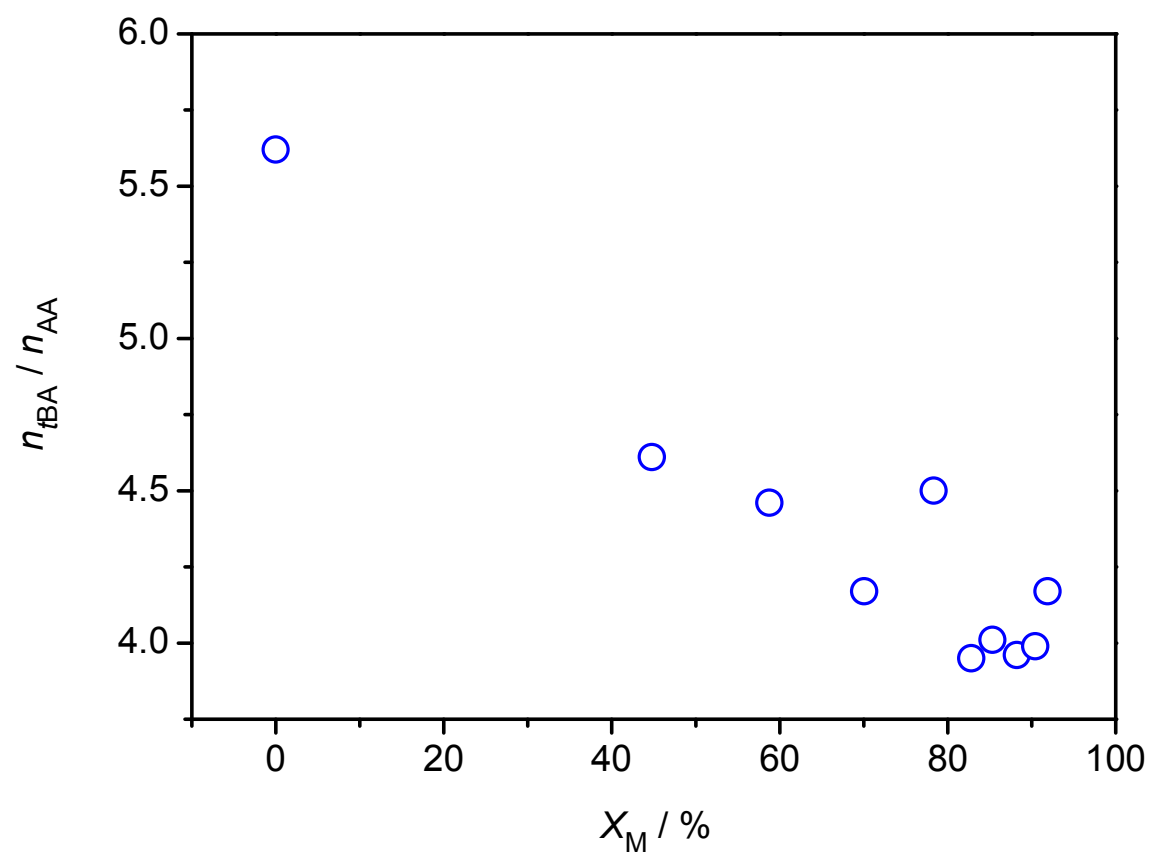

Figure 7-2. Evolution of the molar monomeric ratio of $t \mathrm{BA}$ and $\mathrm{AA}$ in solution $n_{t \mathrm{BA}} / n_{\mathrm{AA}}$ with total monomer conversion $X_{\mathrm{M}}$ determined via ${ }^{1} \mathrm{H}-\mathrm{NMR}$ during RAFT copolymerization of $t \mathrm{BA}$ $\left(3.5 \mathrm{~mol} \cdot \mathrm{L}^{-1}\right)$ and AA $\left(0.62 \mathrm{~mol} \cdot \mathrm{L}^{-1}\right)$ in DMF mediated by DPTH $\left(8.4 \mathrm{mmol} \cdot \mathrm{L}^{-1}\right)$ and initiated by $\operatorname{AIBN}\left(4.0 \mathrm{mmol} \cdot \mathrm{L}^{-1}\right)$ at $60{ }^{\circ} \mathrm{C}$.

Table 7-1. Number-average molar masses $M_{\mathrm{n}}$ of the B- and A-blocks and PDI values for the ABA type $t \mathrm{BA}-\mathrm{AA}$ copolymers.

\begin{tabular}{l|r|r|r}
\hline sample & $\begin{array}{c}M_{\mathrm{n}}^{\text {B-block }} \\
{\left[\mathrm{g} \cdot \mathrm{mol}^{-1}\right]}\end{array}$ & $\begin{array}{c}M_{\mathrm{n}}^{\text {A-block }} \\
{\left[\mathrm{g} \cdot \mathrm{mol}^{-1}\right]}\end{array}$ & PDI \\
\hline $7-1$ & 1068 & 8212 & 1.20 \\
\hline $7-2$ & 1068 & 12604 & 1.25 \\
$7-3$ & 3472 & 8497 & 1.19 \\
$7-4$ & 3472 & 12396 & 1.20 \\
$7-5$ & 3472 & 12781 & 1.24 \\
$7-6$ & 5504 & 10075 & 1.21 \\
$7-7$ & 5504 & 11281 & 1.34 \\
$7-8$ & 5504 & 14184 & 1.22 \\
$7-9$ & 7055 & 10027 & 1.22 \\
$7-10$ & 7055 & 12437 & 1.26 \\
$7-11$ & 9528 & 9390 & 1.34 \\
$7-12$ & 9528 & 9785 & 1.27 \\
\hline
\end{tabular}




\subsection{Results and Discussion}

In the following, the experimental results from DMA are outlined in detail. The subchapter starts by introducing the determined relaxation modes for the $A B A$ type $t \mathrm{BA}-\mathrm{AA}$ copolymers. Afterwards, the dynamic mechanical behavior of the copolymers is compared with pure poly $(t \mathrm{BA})$. Finally, the influence of the Ablock and B-block chain lengths on the temperatures, at which the relaxations and sample-height changes occur, is examined.

\subsubsection{Detected Relaxation Modes}

The measured relaxation spectra of the copolymers exhibit various relaxation modes determined as maxima in $\tan \delta$, of which several can be related to changes in the relative sample height that is simultaneously measured and is given by the ratio of the detected absolute sample height, $h_{\text {sample }}$, and the initial sample height, $h_{\text {sample }}^{0}$. Figure 7-3 displays the spectrum of copolymer 7-7 together with the corresponding relative sample height. The blue curve shows the tangent of the phase angle in a semi-log plot over the temperature. The most eye-catching relaxations are represented by the two distinct peaks in the $\tan \delta$-curve at $313 \mathrm{~K}$ and $396 \mathrm{~K}$, respectively. These peaks are also reflected in the corresponding relative sample height (orange curve) that changes concomitantly. The near room temperature peak is accompanied by a compression of the polymer and can therefore be assigned to a glass transition of the copolymer at $T_{\mathrm{g}}$. After this compression a constant value of the relative sample height in the range of $50 \mathrm{~K}$ is found. At higher temperatures above approximately $375 \mathrm{~K}$ the sample begins to expand and oozes out of the sample hole, which can be seen in the second decrease of the orange curve. This second change in relative sample height of the copolymer coincides with the hightemperature peak in $\tan \delta$ at $396 \mathrm{~K}$.

At temperatures below $T_{\mathrm{g}}$, further maxima and thus relaxation modes can be observed in $\tan \delta$. Between $140 \mathrm{~K}$ and $220 \mathrm{~K}$ a broad $\gamma$-peak can be seen, which is related to a motion of the tert-butyl side groups. ${ }^{[149]}$ Additionally, a secondary relaxation mode (cc-mode) in the range between the first softening and the $\gamma^{-}$ peak in $\tan \delta$ is visible around $260 \mathrm{~K}$. This relaxation is well-reproducible, as depicted in Figure 7-4 for copolymers 7-7 and 7-8, and is absent in pure poly $(t \mathrm{BA})$, as will be shown in the next subchapter. 


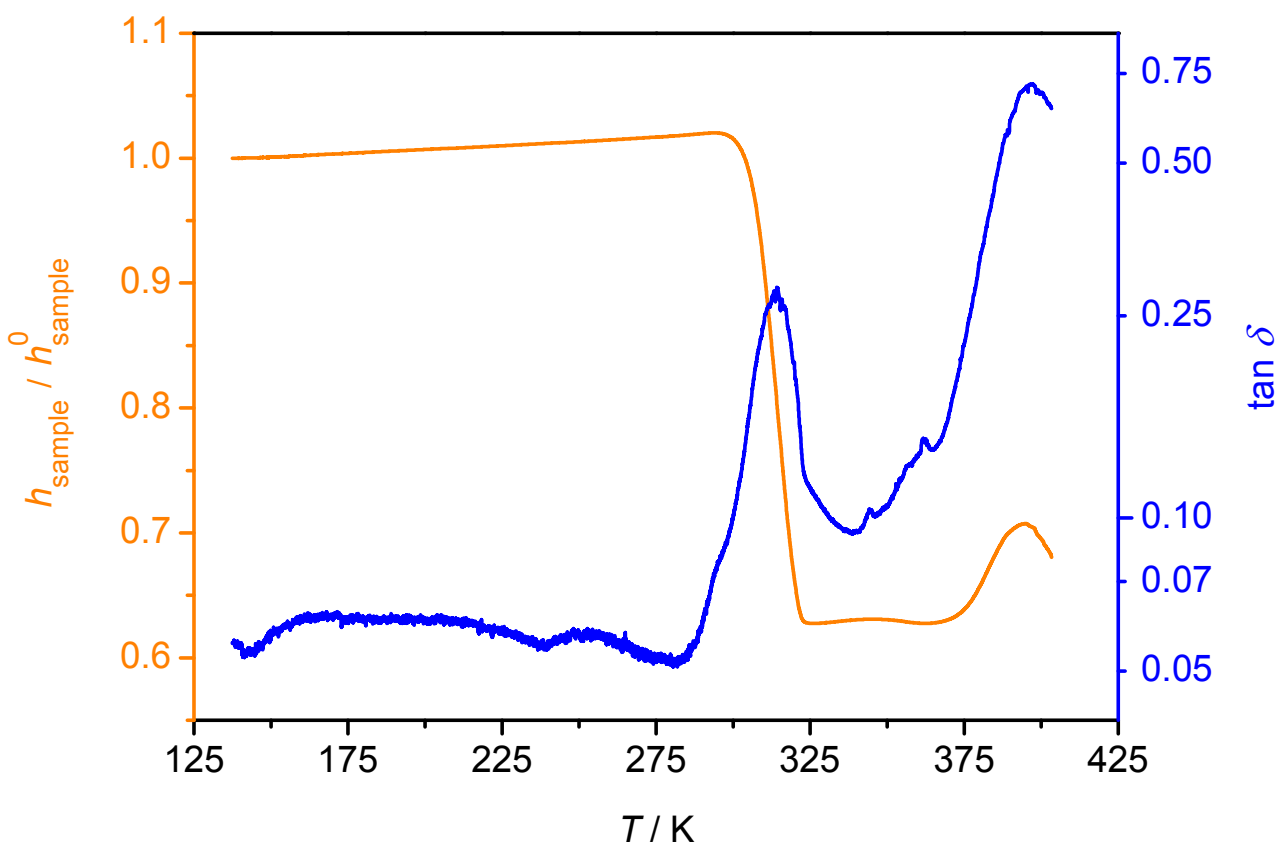

Figure 7-3. Relaxation spectrum (tan $\delta$-curve) and corresponding relative sample height of ABA type copolymer 7-7.

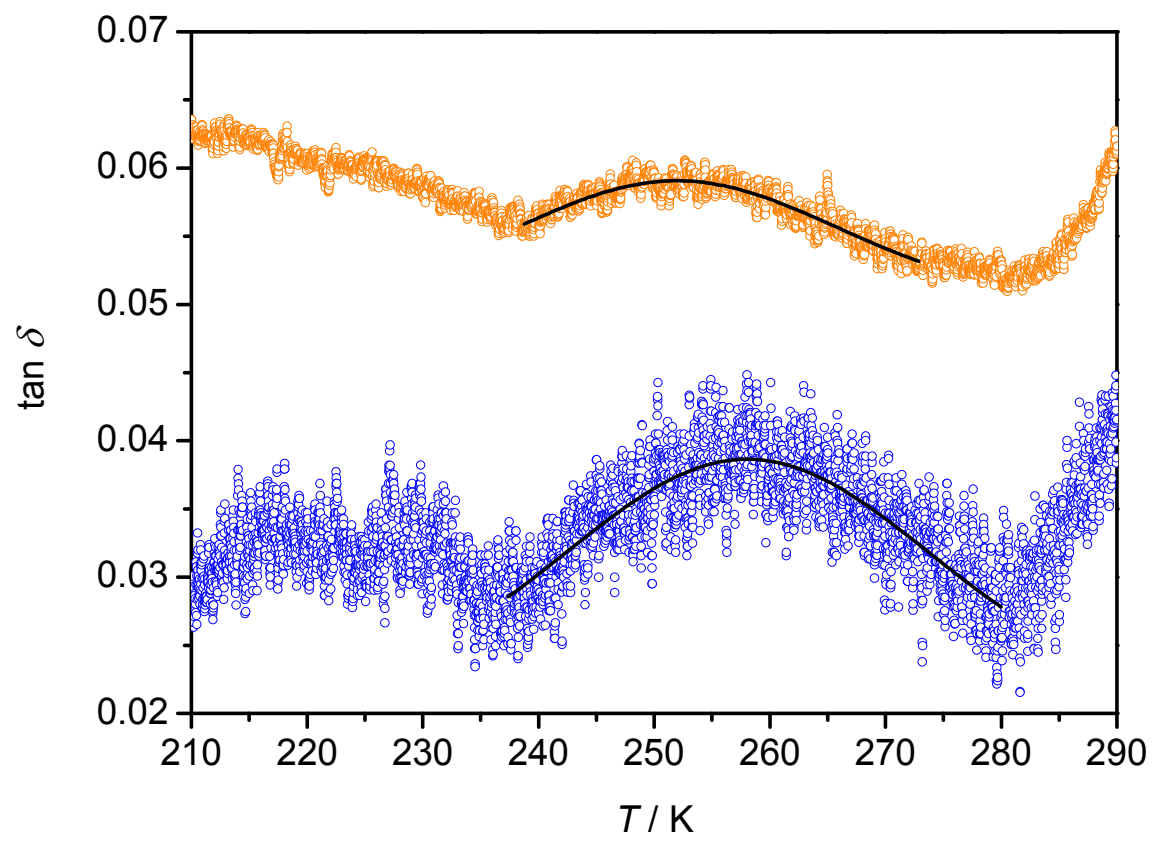

Figure 7-4. Magnification of the chemical confinement mode in the tan $\delta$-curves of ABA type copolymers 7-7 (orange) and 7-8 (blue). 


\subsubsection{Comparison with Pure Poly(tBA)}

In order to extract the relaxation modes that are induced by the insertion of $\mathrm{AA}$ into the outer A-blocks, reference experiments of pure poly $(t \mathrm{BA})$ were performed. Figure 7-5 illustrates the relaxation spectrum and the corresponding relative sample height of a pure poly $(t \mathrm{BA})$ sample in the temperature range of $146 \mathrm{~K}$ to $317 \mathrm{~K}$. As can be seen in the $\tan \delta$-curve, no maximum is present between the $\gamma$-relaxation at low temperatures and the glass transition peak at about $300 \mathrm{~K}$. In comparison to that, the $\tan \delta$-curve of an ABA type copolymer (Figure 7-6), namely sample 7-4, features an additional secondary relaxation similar to samples 7-7 and 7-8 shown in the last subchapter. However, in case of sample 7-4 this relaxation mode is shifted to a lower temperature of approximately $230 \mathrm{~K}$ compared with $250 \mathrm{~K}$ to $260 \mathrm{~K}$ for samples 7-7 and 7-8. This already indicates a pronounced dependence of the cc-relaxation temperature, $T_{\mathrm{cc}}$, on the B-block length, which is covered in more detail later in this chapter. Finally, this secondary relaxation is absent in pure poly $(t \mathrm{BA})$, which is in agreement with works of Cerrada et al. ${ }^{[149]}$

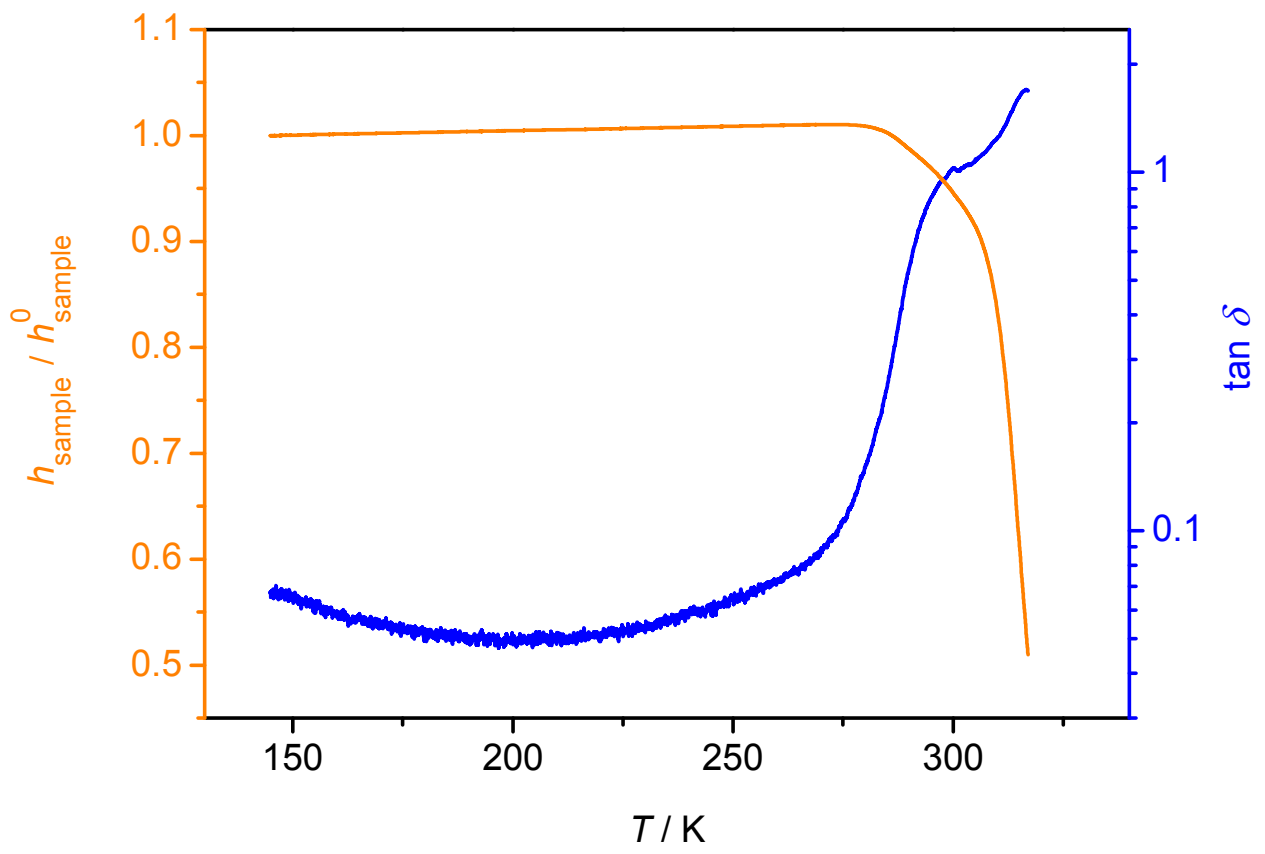

Figure 7-5. Relaxation spectrum (tan $\delta$-curve) and corresponding relative sample height of pure poly $(t \mathrm{BA}), M_{\mathrm{n}}=39647 \mathrm{~g} \cdot \mathrm{mol}^{-1}$.

Other distinct differences are visible in the detected relative sample heights. As can be seen in Figure 7-5, the pure poly $(t \mathrm{BA})$ sample is compressed very 
rapidly above temperatures of about $300 \mathrm{~K}$, whereas the sample height of the copolymer does not change to the same extent (Figure 7-6), suggesting a higher stiffness of the $t \mathrm{BA}-\mathrm{AA}$ copolymer. This difference in flow behavior above the glass transition is even more clearly visible for higher temperatures, as depicted in Figure 7-7. After the compression caused by the glass transition, the copolymer sample features a constant sample height between $325 \mathrm{~K}$ and $375 \mathrm{~K}$ before it expands again and then finally oozes out of the sample hole. This increase in sample height at high temperatures is not observed in the homopolymer samples of $t \mathrm{BA}$, in which case the sample is oozed out immediately after compression. As a conclusion, the high-temperature relaxation observed for the copolymers that coincides with the second softening of the samples is not found for pure poly $(t \mathrm{BA})$. Therefore, the difference in the courses of the relative sample height between pure poly $(t \mathrm{BA})$ and the copolymers at temperatures above $T_{\mathrm{g}}$ is probably caused by hydrogen bonds of the AA units in the A-block, which may stabilize the system in such a way that the polymer sample does not ooze out of the sample holder immediately beyond the glass transition, but remains stiff until the hydrogen bonds are thermally broken.

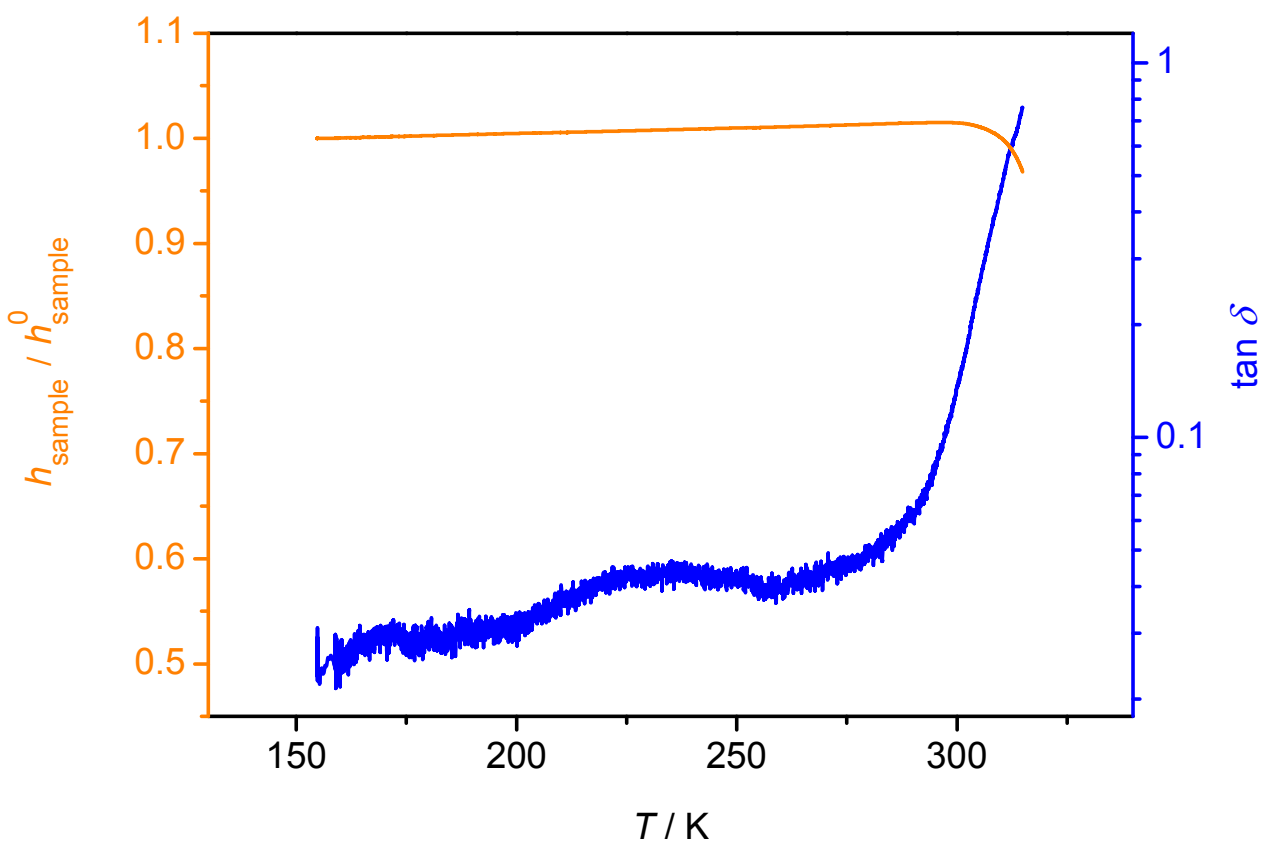

Figure 7-6. Relaxation spectrum (tan $\delta$-curve) and corresponding relative sample height of ABA type copolymer 7-4. 


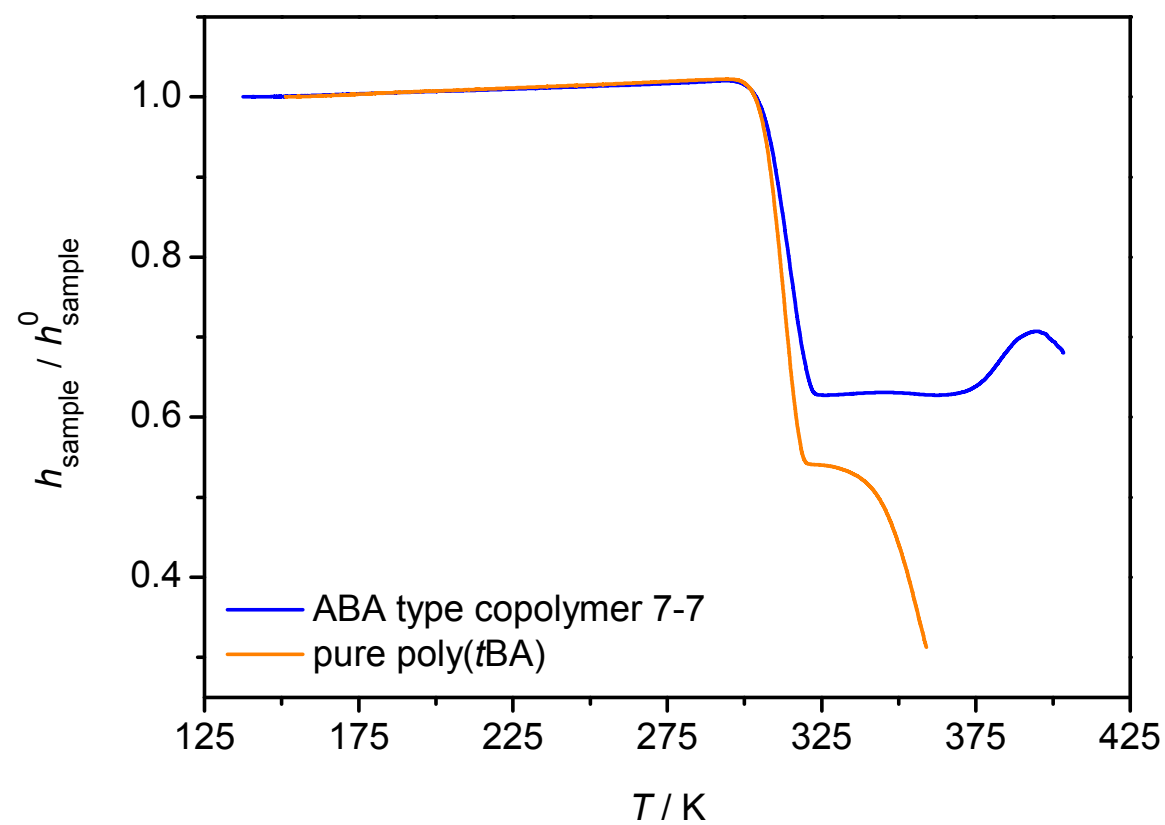

Figure 7-7. Relative sample heights for ABA type copolymer 7-7 and pure poly(tBA) $\left(M_{\mathrm{n}}=18349 \mathrm{~g} \cdot \mathrm{mol}^{-1}\right)$.

\subsubsection{Block-Length Dependencies}

As indicated earlier, the temperatures, at which the observed relaxations and the corresponding onsets of sample-height change occur, exhibit very pronounced chain-length dependencies with respect to the A- and B-blocks, respectively. As the $\gamma$-relaxation determined at very low temperatures is caused by a movement of the tert-butyl side group, no dependency of this relaxation on the two block lengthts was detected.

The temperature of the onset of the first softening, which corresponds to the glass transition temperature of the copolymers, increases with decreasing molar mass of the inner B-block, as shown in Figure 7-8 for four different copolymers that feature three different B-block chain lengths. The complete data, illustrated in Figure 7-9, reveals that the glass transition temperature slightly decreases linearly with increasing molar mass of the inner block with a slope of $2.3 \mathrm{~K}$ per $1000 \mathrm{~g} \cdot \mathrm{mol}^{-1}$. Two feasible explanations are presented in the following: Firstly, this correlation may be explained by considering the relative $t \mathrm{BA}$ content in the polymer. As the glass transition temperature of pure poly $(t \mathrm{BA})$ is apparently lower than for pure poly(AA), which is usually found in the range of about $380 \mathrm{~K},{ }^{[192]}$ longer B-blocks and hence higher $t \mathrm{BA}$ fractions could cause lower $T_{\mathrm{g}}$ values. However, as seen in Figure 7-7, the glass transition temperatures of pure poly $(t \mathrm{BA})$ and an a $t \mathrm{BA}-\mathrm{AA}$ copolymer are similar, which contradicts this 
reasoning. Another possible explanation may be to interpret the first softening not as a glass transition of the entire copolymer but as the glass transition of only the inner B-block. In this case, a higher chain length of the inner block, which is fixed between the hydrogen bonds forming A-blocks, could provide an increased free volume for the glass transition. Consequently, $T_{\mathrm{g}}$ could be lowered, despite the fact that the glass transition temperature generally increases with the molar mass. ${ }^{[193]}$ This, on the other hand, could serve as an explanation for the relatively low $T_{\mathrm{g}}$ values of the copolymers despite the incorporation of $\mathrm{AA}$, as the chain lengths of the B-blocks are smaller compared with the pure poly $(t \mathrm{BA})$ samples. Hence a separate glass transition of the short inner B-blocks should be found at smaller temperatures compared with the poly $(t \mathrm{BA})$ that consists of longer chains according to the molar mass dependence of $T_{\mathrm{g}}$.

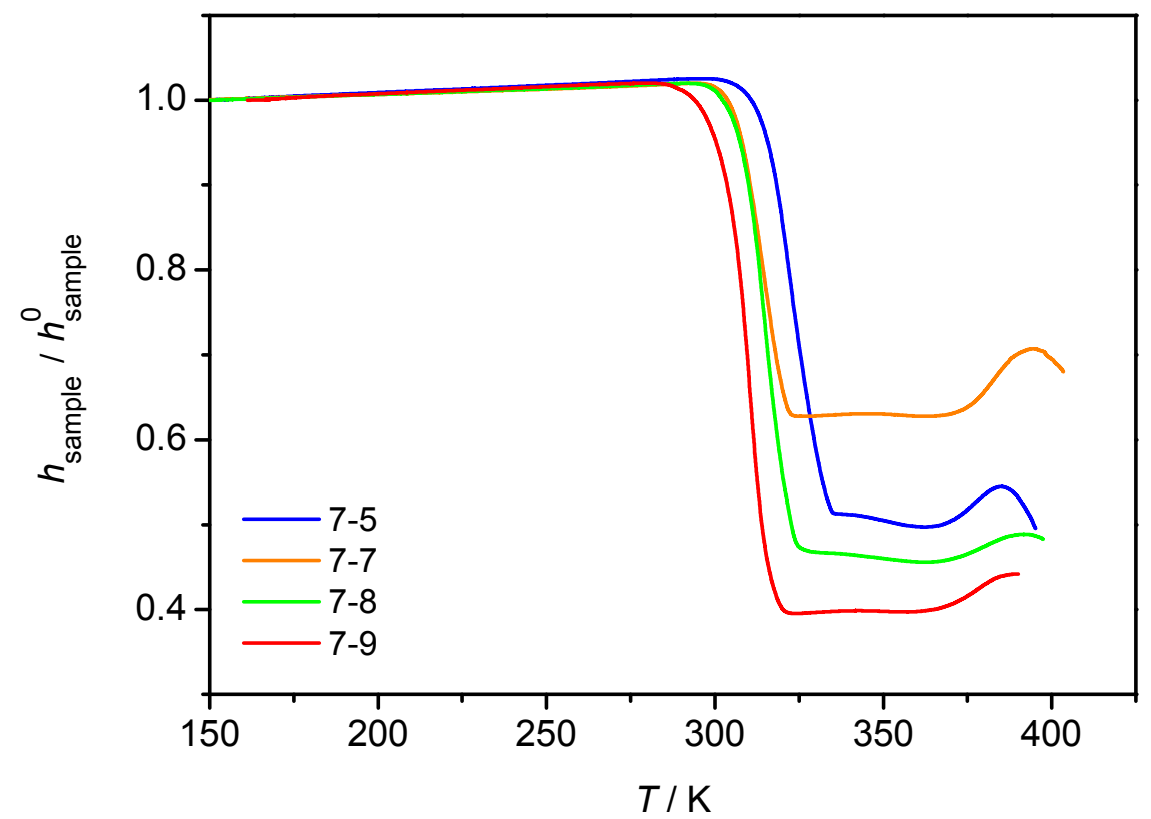

Figure 7-8. Relative sample heights for ABA type copolymers 7-5, 7-7, 7-8 and 7-9.

The temperature of the onset of the second softening, which is observed for the $\mathrm{ABA}$ type $t \mathrm{BA}-\mathrm{AA}$ copolymers at higher temperatures, features a dependency on the molar mass of the outer A-blocks. This can also be seen in Figure 7-8, in which the copolymer with the lowest A-block molar mass of $10027 \mathrm{~g} \cdot \mathrm{mol}^{-1}$ displays a second softening onset of $370 \mathrm{~K}$, whereas for the copolymer with the longest A-blocks with a molar mass of $14184 \mathrm{~g} \cdot \mathrm{mol}^{-1}$ this onset is found at $374 \mathrm{~K}$. From the complete data, depicted in Figure 7-10, the 
chain-length dependence of the second softening temperature can be deduced to be $1.1 \mathrm{~K}$ per $1000 \mathrm{~g} \cdot \mathrm{mol}^{-1}$ with the assumption of a linear correlation. Because this second change in sample height at higher temperature is most likely caused by the AA units in the copolymers, longer A-blocks and thus more AA units could cause the copolymers to remain stiff and to retain a constant sample height up to higher temperatures due to hydrogen bonds. At the point of the second softening, these bonds may finally break thermally and the samples become soft. The expansion of the copolymer samples that occurs in this regime may be caused by a chemical reaction of the tert-butyl side groups stimulated by the AA units, as for example the hydrolysis of the ester moieties. Although the tert-butyl groups in poly $(t \mathrm{BA})$ are known to decompose at approximately $433 \mathrm{~K}$ to yield poly(AA) and isobutylene ${ }^{[194]}$ this reaction does not appear likely as the expansion of the copolymers is detected at significantly lower temperatures. Therefore, a chemical reaction is suggested that is induced by the AA units in the outer A-blocks.

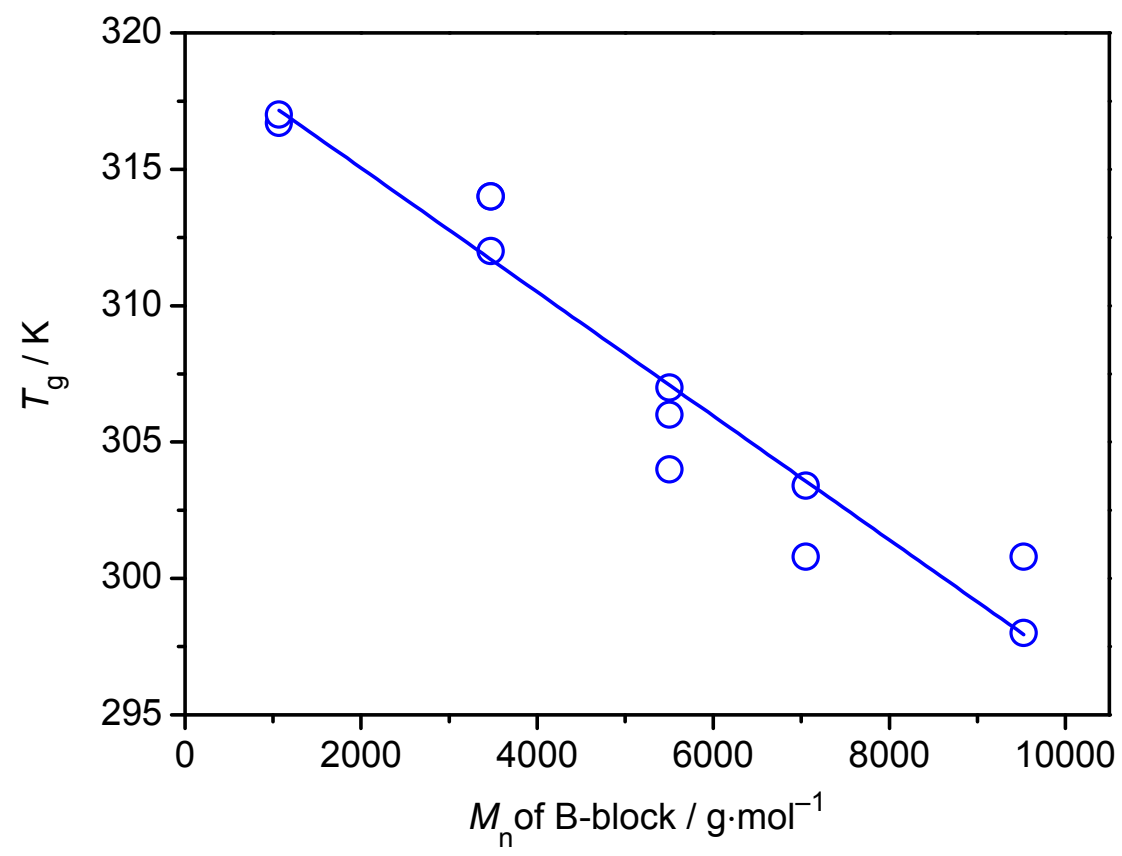

Figure 7-9. Dependence of $T_{\mathrm{g}}$ determined by the first softening onset on the number-average molar mass of the B-block.

The temperatures, at which the secondary cc-relaxation occurs, can be related to the number-average molar masses of the B-blocks similar to the glass transition, although it obeys a contrary slope (Figure 7-11). According to the measured relaxation spectra, the cc-relaxation temperature increases linearly 
with the corresponding length of the confined segment with a slope of $10.6 \mathrm{~K}$ per $1000 \mathrm{~g} \cdot \mathrm{mol}^{-1}$. This behavior may be attributed to a higher amount of energy which is needed to activate the above described flipping movement when the chain length of the moving segment is increased.

Interestingly, a possibly similar relaxation mode, termed $\beta$-relaxation, has been observed by Cerrada and co-workers in poly( $t \mathrm{BA})$-graft-polystyrene that was also located between the $\gamma$-peak and the glass transition. ${ }^{[149]}$ The detected relaxation was shifted to lower temperatures with increasing number of grafted styrene arms and, in agreement with the results of this work, was not observed in the homopolymer of $t \mathrm{BA}$. This relaxation mode was described as movements of the $t \mathrm{BA}$ side groups and its dependence on the number of arms and its absence in pure poly $(t \mathrm{BA})$ were ascribed to lowered steric hindrance in the graft copolymers. However, for the copolymers presented here, the most likely interpretation of this relaxation is a movement of the inner B-block terminally fixed via hydrogen bonds due to the pronounced dependence of the relaxation temperature on the B-block length.

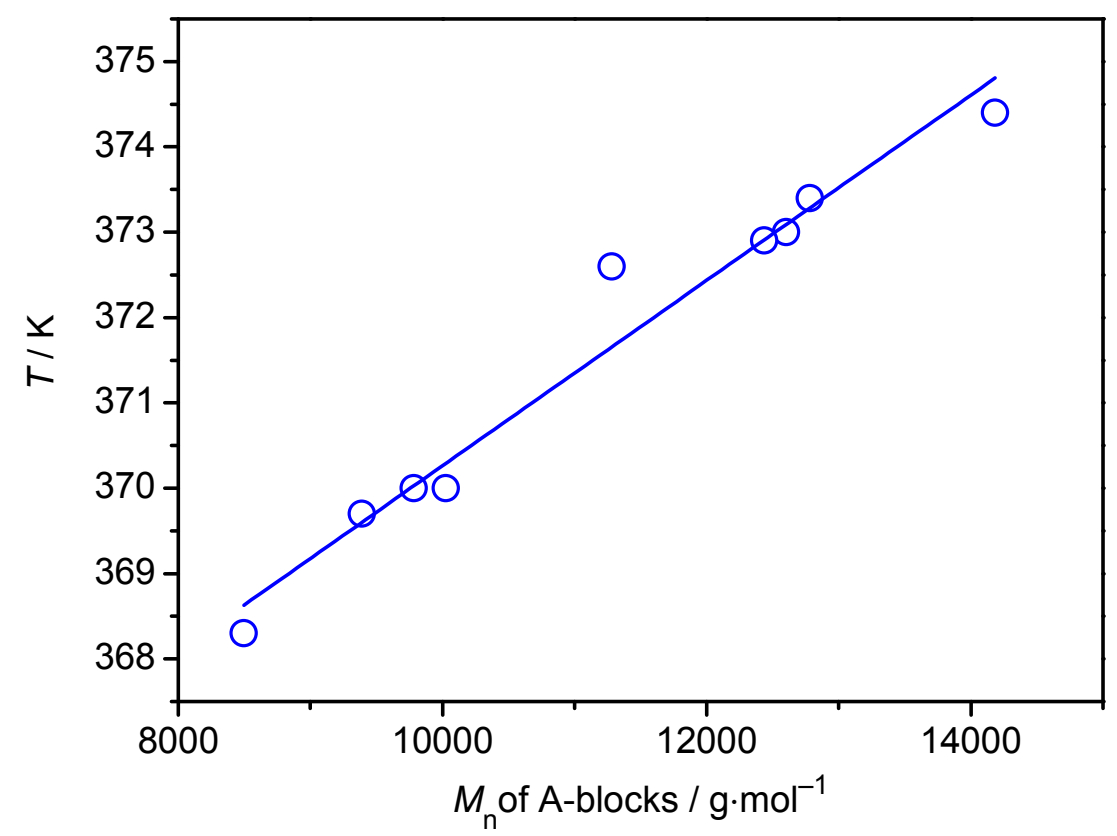

Figure 7-10. Dependence of the second softening onset temperature on the number-average molar mass of the A-blocks. 


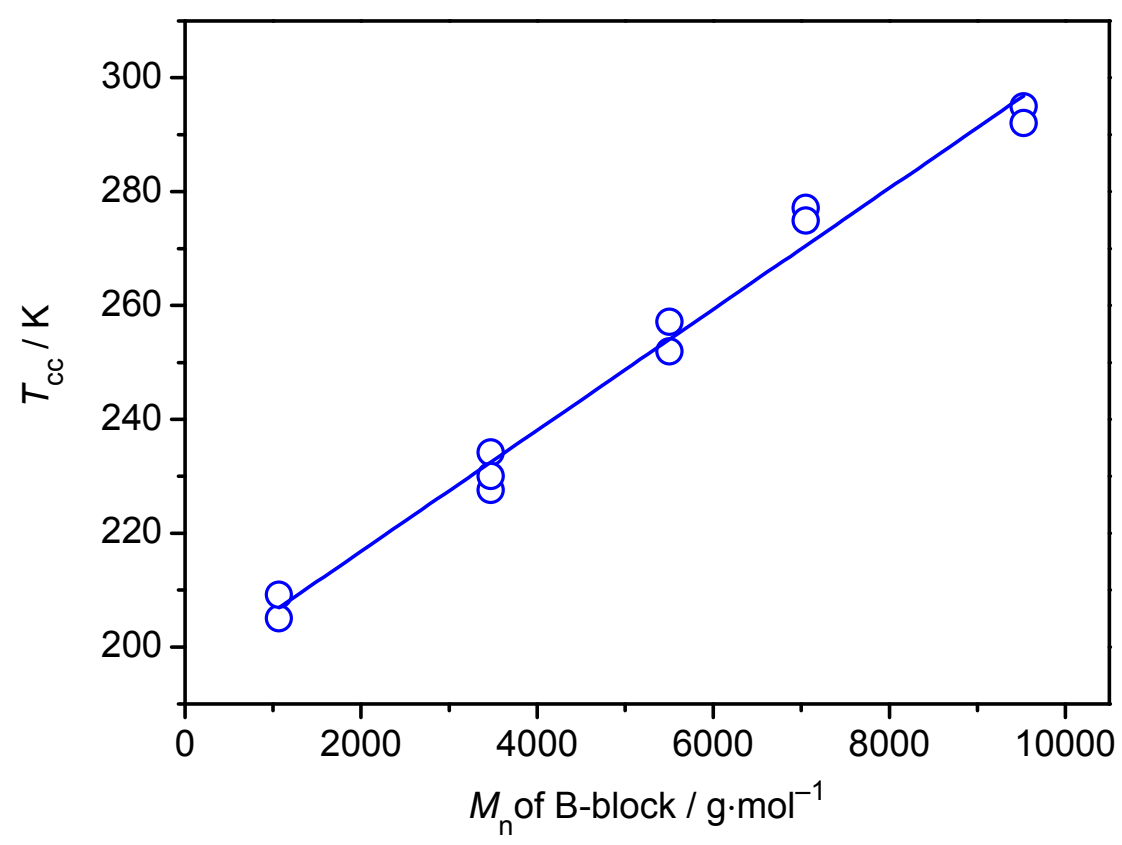

Figure 7-11. Dependence of the cc-relaxation temperature $T_{\mathrm{cc}}$ on the molar mass of the B-block.

\subsection{Conclusion}

Copolymers of $t \mathrm{BA}$ and $\mathrm{AA}$ with an $\mathrm{ABA}$ type structure were synthesized via RAFT Polymerization with control over the molar masses of the A- and B-blocks. The copolymers were subjected to DMA for determining their relaxations, in which the main focus was put on a chemical confinement mode caused by hydrogen bonds. In total, four different relaxation modes could be identified in the relaxation spectra of the ABA type copolymers.

The relaxation close to room temperature could be identified as a glass transition, which was evident from the simultaneous decrease in sample height that indicated a softening of the copolymers. The $T_{\mathrm{g}}$ values obtained from the softening onsets surprisingly shifted to lower temperatures with increasing B-block length. This behavior may be explained by the impact of the $t \mathrm{BA}$ fractions that vary for copolymers which contain B-blocks of different chain lengths. As poly $(t \mathrm{BA})$ features a lower glass transition temperature compared with poly(AA), higher amounts of $t \mathrm{BA}$ for copolymers with longer B-blocks could cause the observed decrease in the $T_{\mathrm{g}}$ values. Alternatively, this relaxation may be regarded as a separate glass transition of the inner B-blocks. In this case, the free volume for the glass transition may be larger for copolymers with longer inner blocks and consequently $T_{\mathrm{g}}$ would be decreased. 
At this stage, the dependence of the detected glass transition on the B-block length is not fully understood and requires further investigations.

At higher temperatures, another relaxation mode was found that was accompanied by a second softening, which, in turn, was preceded by a constant value of the relative sample height. This flow behavior above $T_{\mathrm{g}}$ is largely different from pure poly $(t \mathrm{BA})$, in which case the sample was compressed more rapidly and did not feature a high-temperature relaxation. Therefore, the detected relaxation and softening at higher temperatures probably emerge from the inserted AA units in the outer blocks that additionally enabled the copolymers to resist the applied stresses up to about $50 \mathrm{~K}$ above $T_{\mathrm{g}}$ due to hydrogen bonds. This was further suggested by the increase of the second softening temperature with increasing chain lengths of the A-blocks. Since an increase of the A-block molar masses results in a larger number of AA units and also hydrogen bonds in the copolymers, the temperature at which the copolymers began to flow is consequently proportional to the A-block length.

At low temperatures a $\gamma$-relaxation was observed that involves the movement of the tert-butyl side groups. ${ }^{[149]}$ Between the $\gamma$-relaxation and $T_{\mathrm{g}}$ an additional secondary relaxation was detected that is assigned to a cc-mode. This relaxation was absent in pure poly $(t \mathrm{BA})$ and the temperature, at which this mode occurred, exhibited a pronounced dependence on the molar masses of the inner B-blocks. This secondary cc-relaxation is therefore proposed to be enabled via the introduction of an additional length scale in the polymers. A fixing of the A-blocks, created by hydrogen bonds, produces chemically confined segments of a defined length, namely the B-blocks, which can undergo a similar relaxation as proposed for the ethene-MAA copolymers. ${ }^{[43]}$ However, the copolymers in this thesis were synthesized in a much more controlled fashion with control over the molar masses of the individual blocks, which allows for a more systematic investigation of this novel secondary relaxation mode below $T_{\mathrm{g}}$. The increase of the cc-relaxation temperature with increasing molar masses of the inner blocks could be understood in terms of a larger amount of energy that is required for the movement of longer chain segments. This, in turn, would cause the cc-mode to shift to higher temperatures for longer B-block lengths. 



\section{Tensile Properties of MA-AA Copolymers}

In this chapter, a short insight on the tensile behavior of MA-AA copolymers is given. The results from DMA of $t \mathrm{BA}-\mathrm{AA}$ copolymers, outlined in chapter 7 , have revealed that the defined insertion of $A A$ into the outer chain parts of poly $(t \mathrm{BA})$ largely affects the dynamic mechanical behavior. Hence, the tensile behavior of acrylic polymers doped with AA may also display changes that depend on the AA amount as well as the location of the AA units along the polymer chain.

For application of such copolymers in tensile testing, MA (Scheme 8-1) was used instead of $t \mathrm{BA}$, as poly $(t \mathrm{BA}-c o-\mathrm{AA})$ features a glass transition temperature in the range of $298 \mathrm{~K}$ to $317 \mathrm{~K}$ according to the experiments in this work. Consequently, it is brittle at RT, which hampers the sample preparation for tensile analysis. Pure poly(MA), in turn, exhibits a lower $T_{\mathrm{g}}$ value of about $280 \mathrm{~K}^{[195,196]}$ compared with $T_{\mathrm{g}} \approx 316 \mathrm{~K}$ for pure poly $(t \mathrm{BA}),{ }^{[196]}$ which eases the preparation of tensile specimens that are obtained by cutting samples out of a solution-cast film. The copolymerization parameters for MA and AA were determined to be close to $1.0,{ }^{[197]}$ which assures a steady incorporation of AA during copolymerization.

At the beginning of this chapter, the synthesis and the copolymer samples are introduced. Afterwards, the results from stress-strain analysis are thoroughly examined prior to the conclusion at the end of the chapter.

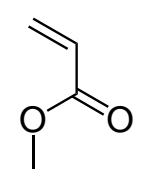

Scheme 8-1. Chemical structure of MA. 


\subsection{Synthesis}

Three different MA-AA copolymers were synthesized via RAFT polymerization mediated by the bifunctional trithiocarbonate DPTH that differ mainly with regard to their AA content (Table 8-1), which was determined via NMR analysis of the copolymers by comparing the signal of the three protons of the methyl side group of MA between approximately $3.4 \mathrm{ppm}$ and $3.8 \mathrm{ppm}$ with the signal of the complete backbone protons between about $0.6 \mathrm{ppm}$ and $2.8 \mathrm{ppm}$. In addition to that, the copolymer with intermediate AA content was synthesized similar to the ABA type $t \mathrm{BA}-\mathrm{AA}$ copolymers presented in subchapter 7.1, that is pure poly(MA) was synthesized first to establish a pure inner MA-block that was subsequently subjected to a chain extension polymerization with a mixture of MA and AA. This approach may allow for extracting specific tensile properties that emerge from the presence of the AA only at the outer chain parts, which could supplement the DMA results for the ABA type $t \mathrm{BA}-\mathrm{AA}$ copolymers presented in chapter 7.

Because of the hydrophilic character of the MA-AA copolymers, their $M_{n}$ values were determined on an SEC system that uses $N, N$-dimethylacetamide (DMAc) as the eluent. However, at the time of experiment no calibrations for either poly(MA) or poly(AA) were available for this SEC setup, which is why a polystyrene calibration was applied instead. Therefore, the molar-mass data of the three copolymers only allows the relative comparison of the three copolymers. The number-average molar mass of the inner pure poly(MA)-block of copolymer 8-3, however, was obtained on a THF-SEC system with the correct calibration and hence reflects a more reliable value for $M_{\mathrm{n}}$.

Table 8-1. Copolymer types, number-average molar masses $M_{\mathrm{n}}$, and molar AA contents of the three MA-AA copolymers.

\begin{tabular}{l|l|r|c}
\hline sample & $\begin{array}{c}\text { copolymer } \\
\text { type }\end{array}$ & \multicolumn{1}{c}{$\begin{array}{c}M_{\mathrm{n}} \\
{\left[\mathrm{g} \cdot \mathrm{mol}^{-1}\right]}\end{array}$} & $\begin{array}{c}F_{\mathrm{AA}} \\
{[\%]}\end{array}$ \\
\hline $\mathbf{8 - 1}$ & random & 40886 & 5.66 \\
$\mathbf{8 - 2}$ & random & 45039 & 13.4 \\
$\mathbf{8 - 3}$ & ABA & B-block: 23000 & 8.78 \\
& & Total: 48503 & \\
\hline
\end{tabular}




\subsection{Results and Discussion}

The tensile properties that were extracted from the stress-strain curves are listed in Table 8-2 and Table 8-3. From each copolymer, three to five specimens were subjected to tensile testing to assure reproducibility. The stress-strain plots of copolymer 8-1 with the lowest AA content of $5.66 \%$, depicted in Figure 8-1, displays a very wide plastic range beyond a pronounced yield point. The apparent stresses first drop after the yield point to about $50 \%$, increase again and finally level off shortly before the break point. The determined elongations at break were high with an average value of $956.2 \%$, which further resulted in a large average value for the toughness of the copolymer of $58 \mathrm{MJ} \cdot \mathrm{m}^{-3}$.

Table 8-2. Young's moduli $E$, elongations and stresses at break $\varepsilon_{\mathrm{B}}$ and $\sigma_{\mathrm{B}}$, and the corresponding sample standard deviations of the three MA-AA copolymers.

\begin{tabular}{l|r|r|c|r|r|r}
\hline sample & $\begin{array}{c}E \\
{[\mathrm{MPa}]}\end{array}$ & $\begin{array}{c}\Delta E \\
{[\mathrm{MPa}]}\end{array}$ & $\begin{array}{c}\varepsilon_{\mathrm{B}} \\
{[\%]}\end{array}$ & $\begin{array}{c}\Delta \varepsilon_{\mathrm{B}} \\
{[\%]}\end{array}$ & $\begin{array}{c}\sigma_{\mathrm{B}} \\
{[\mathrm{MPa}]}\end{array}$ & $\begin{array}{c}\Delta \sigma_{\mathrm{B}} \\
{[\mathrm{MPa}]}\end{array}$ \\
\hline $\mathbf{8 - 1}$ & 496 & 25 & 956.2 & 87.0 & 6.75 & 0.39 \\
$\mathbf{8 - 2}$ & 31 & 6 & 474.2 & 34.3 & 2.20 & 0.29 \\
$\mathbf{8 - 3}$ & 736 & 35 & 373.9 & 96.2 & 5.38 & 3.96 \\
\hline
\end{tabular}

Table 8-3. Strains and stresses at yield point $\varepsilon_{\mathrm{Y}}$ and $\sigma_{\mathrm{Y}}$, toughnesses $\tau$, and the corresponding sample standard deviations of the three MA-AA copolymers.

\begin{tabular}{l|r|r|r|r|r|r}
\hline sample & $\begin{array}{c}\varepsilon_{\mathrm{Y}} \\
{[\%]}\end{array}$ & $\begin{array}{c}\Delta \varepsilon_{\mathrm{Y}} \\
{[\%]}\end{array}$ & $\begin{array}{c}\sigma_{\mathrm{Y}} \\
{[\mathrm{MPa}]}\end{array}$ & $\begin{array}{c}\Delta \sigma_{\mathrm{Y}} \\
{[\mathrm{MPa}]}\end{array}$ & $\begin{array}{c}\tau \\
{\left[\mathrm{MJ} \cdot \mathrm{m}^{-3}\right]}\end{array}$ & $\begin{array}{c}\Delta \tau \\
{\left[\mathrm{MJ} \cdot \mathrm{m}^{-3}\right]}\end{array}$ \\
\hline $\mathbf{8 - 1}$ & 5.1 & 0.5 & 7.47 & 0.62 & 58 & 7 \\
$\mathbf{8 - 2}$ & 13.7 & 3.4 & 0.912 & 0.012 & 7.5 & 0.7 \\
$\mathbf{8 - 3}$ & 5.0 & 0.3 & 14.7 & 1.7 & 31 & 9 \\
\hline
\end{tabular}




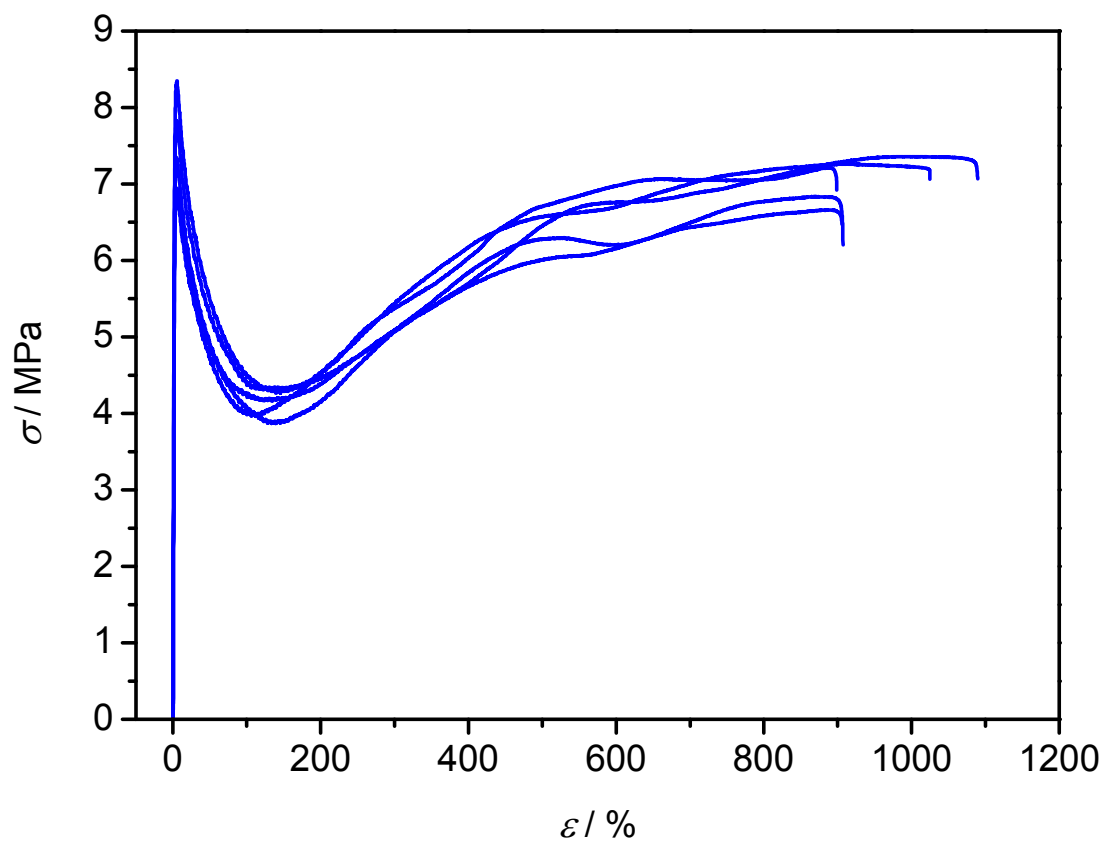

Figure 8-1. Stress-strain curves of five specimens of MA-AA copolymer 8-1.

The tensile curves of copolymer 8-2 (Figure 8-2) with an AA content of $13.4 \%$ display significantly different behaviors compared with the samples of 8-1. For none of the measured curves the yield point occurs as a maximum stress, but rather as the point where the stress-strain curve levels off. In the plastic regime, the apparent stresses increase steadily up to the break point. Surprisingly, the Young's modulus is largely decreased, although the amount of $\mathrm{AA}$ and thus also the number of hydrogen bonds and cross-linking points should be higher. In addition to that, the stresses at yield and break point, the strain at break as well as the toughness of the copolymers are largely reduced compared with copolymer 8-1. The only exception is the strain at yield, which is $8.6 \%$ higher than $\varepsilon_{\mathrm{Y}}$ from sample 8-1. Therefore, at least in the case of a random insertion of $\mathrm{AA}$, the increase of this monomer content from $5.66 \%$ to $13.4 \%$ rather seems to diminish the tensile properties of the copolymers. 


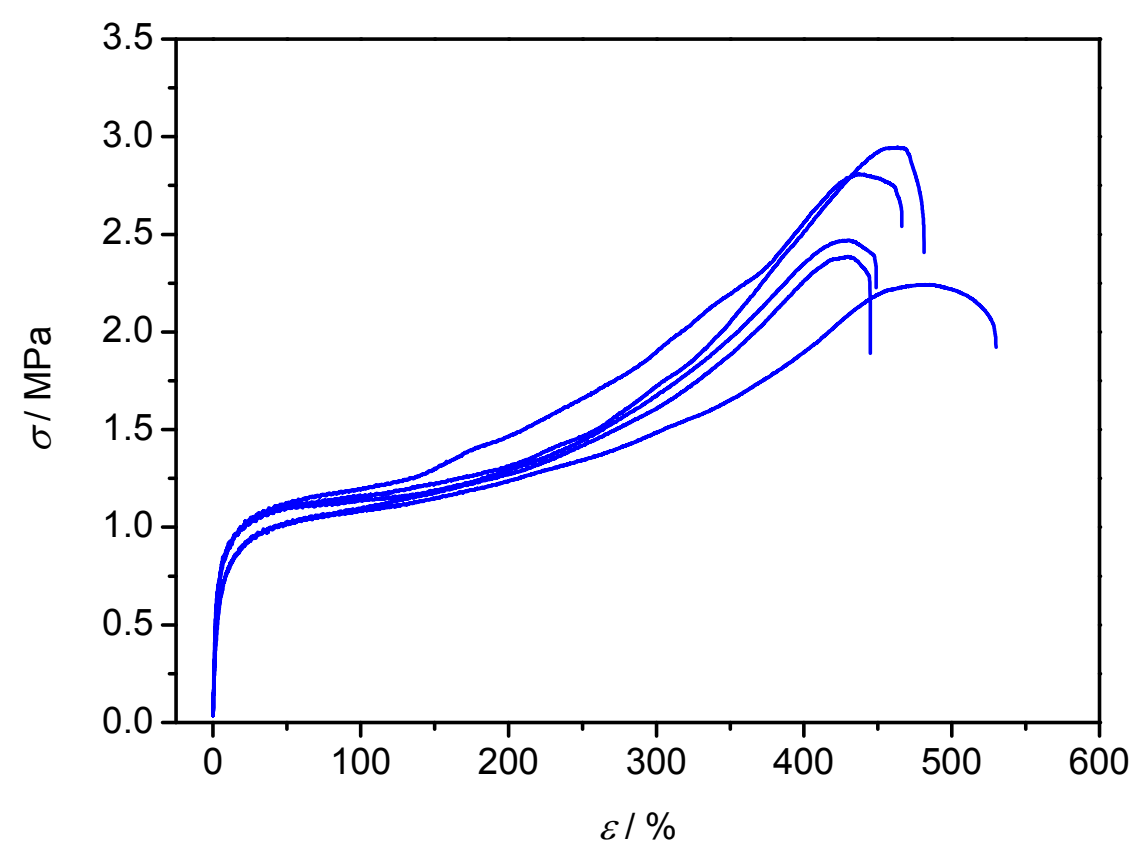

Figure 8-2. Stress-strain curves of five specimens of MA-AA copolymer 8-2.

The stress-strain curves of copolymer 8-3 with an intermediate AA content of $8.78 \%$, shown in Figure 8-3, resemble some characteristics of sample 8-1, since they also exhibit a pronounced yield point seen as a maximum stress and a subsequent drop in apparent stress of about $50 \%$. Afterwards, the stresses increase gradually, though without levelling, until the break point. The latter is characterized by lower $\varepsilon_{\mathrm{B}}$ and $\sigma_{\mathrm{B}}$ values compared with the parameters from copolymer 8-1. The average toughness of the copolymer is in between copolymers 8-1 and 8-2 with $31 \mathrm{MJ} \cdot \mathrm{m}^{-3}$. Interestingly, the determined average $E$ value and the yield strength $\sigma_{\mathrm{Y}}$ are both significantly higher with values of $736 \mathrm{MPa}$ and $14.7 \mathrm{MPa}$, respectively, compared with samples 8-1 and 8-2. The samples from copolymer 8-3 thus feature a higher stiffness and are able to withstand higher stresses compared with samples from 8-1 and 8-2 before permanent deformations take place. This finding may be related to the higher stiffness of the ABA type $t \mathrm{BA}-\mathrm{AA}$ copolymers, determined by DMA in chapter 7, which resulted from the introduction of AA into the outer A-blocks. The concentration of AA in the outer parts of the polymer chain could result in a more effective and stable network of hydrogen bonds and thus cross-links between the macromolecules. Consequently, this may enhance selected tensile properties compared with a random distribution of the hydrogen bond forming comonomer. 


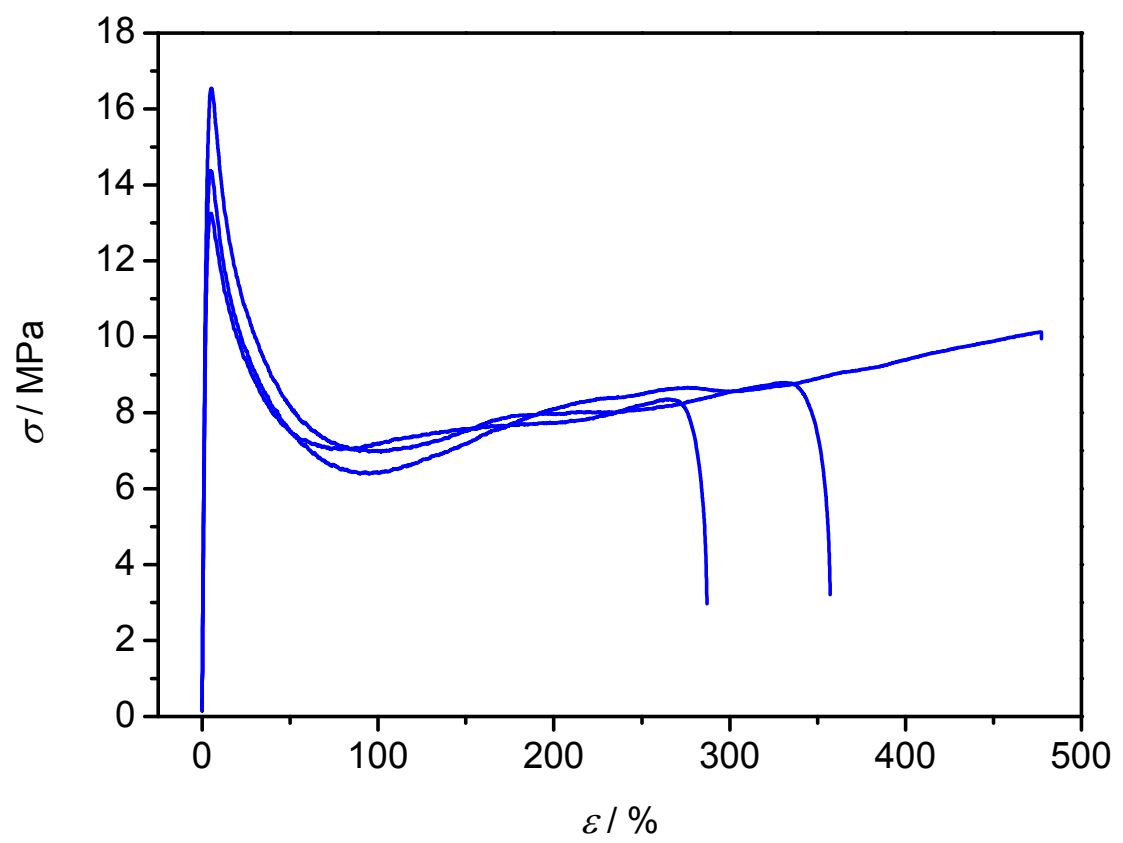

Figure 8-3. Stress-strain curves of three specimens of MA-AA copolymer 8-3.

\subsection{Conclusion}

The amount of AA in poly(MA-co-AA) largely affects the tensile behavior. In case of a low molar AA fraction of $5.66 \%$, the copolymer exhibits a pronounced yield point and very high elongations at break. An increase of the AA content to $13.4 \%$ changes the tensile behavior dramatically, which was evident from significantly reduced $E, \varepsilon_{\mathrm{B}}, \sigma_{\mathrm{B}}, \sigma_{\mathrm{Y}}$ and $\tau$ values. Furthermore, the yield point was not displayed as a point of maximum stress but rather as the point where the stress-strain curve levels off.

The copolymer with an ABA type structure, in which case the AA is only inserted in the outer chain parts, and an intermediate AA fraction of $8.78 \%$ displayed partially improved tensile properties. Specifically the parameters $E$ and $\sigma_{\mathrm{Y}}$ were significantly higher compared with the two copolymers that feature a random distribution of AA along the polymer chain. The concentration of $\mathrm{AA}$ at the chain ends may enable a more effective network of hydrogen bonds, which in turn results in a higher tensile modulus and improved yield strength. These tensile properties are both highly important for qualifying such polymers as materials for applications. However, as these results merely rely on three copolymers that do not have identical AA contents, more systematic stress-strain analysis needs to be performed in order to gain a more detailed comprehension for the interplay of the copolymer structure, 
especially regarding the distribution of the $\mathrm{AA}$, and the resulting tensile properties. 



\section{Closing Remarks}

A wide range of macromolecular research has been covered in this work. This includes the kinetics and mechanism of surface-initiated (RAFT) polymerizations, which have further been applied for producing silica-filled styrene- $n \mathrm{BA}$ copolymers for tensile analysis. Moreover, the relaxation processes of ABA type $t \mathrm{BA}-\mathrm{AA}$ copolymers, synthesized by RAFT polymerization, were determined by DMA. These studies were complemented by tensile testing of related MA-AA copolymers. The thesis in hand thus bridges the fields of polymerization kinetics, macromolecular architecture and the polymer's mechanical behavior.

For the first time, propagation rate coefficients for surface-initiated radical polymerizations were obtained by application of a surface-grafted photoinitiator in PLP-SEC. This straightforward approach enables insight into radical propagation kinetics from a solid support, which could only be indirectly assessed beforehand. ${ }^{[33]}$ The determined $k_{\mathrm{p}}$ values presented in this thesis (chapter 4$)^{[152]}$ are substantially higher than given IUPAC data. ${ }^{[67,155]}$ Possible causes are presented such as a reduced shielding of the radical site, which could be related to the chain-length dependence of $k_{\mathrm{p}}{ }^{[181]}$

In order to gain a more comprehensive knowledge of propagation rate coefficients for polymerizations from surfaces, additional systematic measurements need to be performed with different monomers, concentrations and at varying temperatures. Since the determination of $k_{\mathrm{p}}$ via PLP-SEC relies on SEC analysis, which is inherently subject to statistical errors, more $k_{\mathrm{p}}$ data is necessary to confirm the present results and to expand the database of propagation rate coefficients determined by the IUPAC task group. Another important aspect is the grafting density of growing chains, which is particularly crucial for surface-initiated polymerizations. This parameter could be controlled through the adjustment of the initiator loading on the silica particles and should hence be varied as well in future experiments. This could allow the comparison of $k_{\mathrm{p}}$ values that result from different density and thus conformation regimes, such as coil-like and brush-like orientations of anchored 
macroradicals. ${ }^{[179]}$ Such knowledge could prove beneficial also for other studies on surface-initiated polymerizations that use the grafting-from method as the R-group approach in RAFT graft polymerization. Finally, beside the surfaceimmobilized initiator an additional free initiator in the interstitial solution phase could be employed, which might also affect the obtained $k_{\mathrm{p}}$ values due to further termination mechanisms. Following this approach, the quality of the overall termination rate for the exclusive termination by grafted radicals may be assessed, which is requested to take place almost instantly in PLP-SEC to produce well-structured MMDs. This way, possible side effects, as for example an extended growing period caused by slowed chain-end encounter processes on the surface prior to termination, may be examined. Such effects may arise from the restricted movement of the anchored radicals formed by the photoinitiator compared with free radicals in solution.

The thermally surface-initiated polymerization of styrene with and without additional RAFT agent in solution allowed detailed insight into the molar-mass evolution of free and fixed polystyrene, the polymer loading on the silica particles and its effect on the surface topography of the silica-styrene hybrids (chapter 5). In general, the accurate prediction of the chain-length relation between free and anchored polymer still remains a major challenge when performing such polymerizations. ${ }^{[35,36]}$ This partially arises from the complex interaction of polymerization reactions that take place (i) in solution, (ii) on the surface and (iii) in between the two regions. In case of the conventional radical surface-initiated polymerization of styrene presented in this work, the generation of grafted polystyrene was accompanied by the formation of free polystyrene that is caused by transfer reactions of anchored radicals to solution species and featured significantly higher molar masses than the anchored chains. This observation may be attributed to a very low radical concentration in solution that is expected because of the low transfer rate coefficient of styrene relative to, for example, $\mathrm{MA},{ }^{[198]}$ in which case a contrary relationship of molar masses in solution and on the surface was detected. ${ }^{[35]}$

In contrast to the conventional radical approach, the addition of RAFT agent to the solution induced living behavior both on the surface and in solution. Although the two types of polymers exhibited similar molar-mass evolutions, distinct differences in their MMDs were visible that could be partially reconstructed and identified by PREDICI ${ }^{\circledR}$ simulations. The best match of the MMD data was obtained by application of the slow fragmentation model ${ }^{[102]}$ and a fitted addition rate coefficient for the main equilibrium between surface and solution species of $k_{\text {adoL }}=0.5 \cdot k_{\text {ad }}$. However, even in this case the simulated MMDs exhibit distinct deviations from the experimental MMDs, particularly regarding the magnitudes of the molar-mass shoulders of the MMDs for the free and the attached polystyrene. Hence additional investigations are required, which should include a surface-initiated RAFT polymerization without kinetic 
side effects, such as rate retardation, to reduce the amount of uncertainties in the PREDICI ${ }^{\circledR}$ models. This could be easily achieved by using another RAFT agent as for instance a trithiocarbonate. The decomposition kinetics of the immobilized azo initiator ACTA, which is still unknown at this stage, is to be determined in order to apply more realistic values for the decomposition of the initiator molecules. As the developed PREDICI ${ }^{\circledR}$ models allow for the separation of initiator-derived and R-group-derived polymer chains, they can also be applied to other RAFT polymerizations where the distinction between the two sorts of polymer is a relevant issue.

The surface properties of the silica-polystyrene hybrids were probed with SEM, which has proven to detect changes in the surface topography that arise from variations in the polymer loading on the particles and number-average molar mass of the anchored macromolecules. ${ }^{[35,39]}$ As illustrated in subchapter 5.1.3, the surface of the polymer-modified silica was substantially different from pure fumed silica and exhibited a gradual change upon increasing polystyrene content on the surface. In addition to that, the polystyrene-silica composite that contained a maximum amount of grafted polymer displayed several eyecatching filaments of approximately $1 \mu \mathrm{m}$ in length at the site of cracks, which were probably caused by an ensemble of anchored polystyrene chains. Although SEM constitutes a well-suited method for surface analysis of such materials, it shall be complemented by other techniques in upcoming works as for example atomic force microscopy (AFM) ${ }^{[199]}$ ellipsometry ${ }^{[200]}$ and stimulated emission depletion (STED) microscopy. ${ }^{[201]}$ The latter may additionally allow insight into the initiator decay on the surface by reactions of the radicals with a dye, which is subsequently detected via STED microscopy and could thus enable the estimation of the fraction of decomposed initiator fragments.

The surface-initiated polymerization method has been successfully applied to produce silica-filled styrene- $n \mathrm{BA}$ copolymers for tensile analysis. This approach allows for the one-step synthesis of silica-polymer hybrids, in which the particles are modified with polymer during the polymerization to enhance the compatibility between the polymer matrix and the silica filler. This is a highly relevant issue for the application of fillers for macromolecular materials, as the pure silica particles are in most cases not compatible with the polymer because of their hydrophilic character, which causes agglomeration of the particles and hampers the effective improvement of the mechanical properties. ${ }^{[186]}$ This incompatibility of pure silica is also evident from subchapter 6.2.2, which showed that the elongation at break and the toughness of such a composite are substantially diminished despite a low amount of attached copolymer on the silica surface determined by TGA. The detection of copolymer on the surface of pure silica particles after RAFT polymerization is quite astonishing, but could be attributed to the physisorption of copolymer chains caused by the polar $n \mathrm{BA}$ monomer units. This suggestion may be 
checked by polymerization of non-polar monomers, such as styrene, in the presence of pure silica particles, in which case no surface-attached polymer is expected.

The synthesis routine shown in this work opens up avenues to produce nanocomposites that not only display enhanced interactions between the polymer matrix and the filler, but can also be tuned with regard to crucial parameters such as silica content, grafting density of anchored polymer chains and their molar mass. The latter may be achieved by the employment of RAFT polymerization, which can also be used to yield block-copolymer structures on the surface by application of anchored RAFT agents as for example the anchored trithiocarbonate PTPT. This approach appears to be particularly promising, since the corresponding random styrene- $n \mathrm{BA}$ copolymer filled with PTPT-modified silica particles featured the best overall tensile properties compared with alternative silica fillers used in this work due to the high content of anchored poly(styrene-co- $n \mathrm{BA})$ of $83 \%$ on the silica particles (subchapter 6.2.2). In future studies, the molar masses of the grafted copolymers are to be determined by SEC, since not only the polymer loading on the silica surface but also the chain length may largely affect the interaction between the filler particles and the matrix polymer. The favorable damping properties of random styrene- $n \mathrm{BA}$ copolymers ${ }^{[188]}$ also qualify such composites for DMA, as it directly yields the damping behavior of the material and may thus reveal further effects that are caused by the amount and type of silica filler in the copolymers. Finally, size and dispersion of the silica nanoparticles embedded in the polymer matrix could be evaluated by characterization of silica-polymer composite films by transmission electron microscopy (TEM). ${ }^{[202]}$

DMA of ABA type $t \mathrm{BA}-\mathrm{AA}$ copolymers demonstrated the significant impact of hydrogen bonds on the relaxation processes of poly $(t \mathrm{BA})$. This tailoring of dynamic mechanical properties can most certainly be applied to other types of polymers as well. Therefore, such DMA measurements may be extended to copolymers with variation of characteristics such as monomeric composition (e.g. the concentration of hydrogen bonds), molar mass and constitution. Recently, multiblock copolymers of styrene and $n \mathrm{BA}$ were successfully synthesized via the RAFT technique, ${ }^{[203,204]}$ which may enable the preparation of copolymers that contain a multitude of chemically confined segments in one macromolecular chain compared with only one confined segment in the ABA type copolymers described in this thesis. This would also more accurately resemble the structure of the ethene-MAA copolymers, in which the chemicalconfinement mode was originally detected via DMA. ${ }^{[43]}$

On the basis of the relaxations observed in $t \mathrm{BA}-\mathrm{AA}$ copolymers, the influence of the AA content and the type of insertion were probed for copolymers of MA and AA in tensile testing. The results show that the AA content has a major impact on the tensile properties of the copolymers. By 
incorporating the AA in the outer parts of the polymer chain, the Young's modulus and the yield strength could be substantially increased. This result may be related to the increased stability of the $t \mathrm{BA}-\mathrm{AA}$ copolymer towards compression detected by DMA. A concentration of hydrogen bonds in the outer parts of the polymer chain may form a more effective network of cross-links that also enhances the tensile properties. However, the determination of structure-property relationships remains challenging due to the large amount of parameters that influence the tensile behavior. ${ }^{[4]}$ For this reason, experiments are needed in which ideally only one structural parameter is varied systematically to establish a correlation between molecular structure and mechanical properties. In case of the MA-AA copolymers, especially the AA content ought to be equal, as differences in the AA fraction may otherwise overlap impacts that emerge from structural features such as the ABA type constitution. 



\section{Experimental Part}

In this chapter, the chemicals used in this work, particularly monomers, initiators, and RAFT agents, are described. In addition to that, all experimental procedures, the instrumentation, and the performed simulations are covered.

\subsection{Chemicals}

\subsubsection{Monomers}

Styrene $\left(M=104.15 \mathrm{~g} \cdot \mathrm{mol}^{-1}\right.$, Aldrich, $\geq 99 \%$, stabilized with $10-15 \mathrm{ppm}$ 4-tertbutylcatechol), $n \mathrm{BA}\left(M=128.17 \mathrm{~g} \cdot \mathrm{mol}^{-1}\right.$, Fluka, $\geq 99.5 \%$, stabilized with $15 \mathrm{ppm}$ hydroquinone monomethyl ether), $t \mathrm{BA}\left(M=128.17 \mathrm{~g} \cdot \mathrm{mol}^{-1}\right.$, Aldrich, $98 \%$, stabilized with 10-20 ppm hydroquinone monomethyl ether), and MA ( $M=$ $86.09 \mathrm{~g} \cdot \mathrm{mol}^{-1}$, Aldrich, $99 \%$, stabilized with $\sim 100 \mathrm{ppm}$ hydroquinone monomethyl ether) were passed through a basic alumina (Fluka, Brockmann activity I) column before use to remove the inhibitor. AA $\left(M=72.06 \mathrm{~g} \cdot \mathrm{mol}^{-1}\right.$, Merck, $\geq 99 \%$, stabilized with hydroquinone monomethyl ether) was distilled through a Vigreux column at approximately 10 mbar over copper fibres. All monomers were stored at $3{ }^{\circ} \mathrm{C}$.

\subsubsection{Initiators}

DMPA $\left(M=256.30 \mathrm{~g} \cdot \mathrm{mol}^{-1}\right.$, Aldrich, $\left.99 \%\right)$ was used as received. AIBN $\left(M=164.21 \mathrm{~g} \cdot \mathrm{mol}^{-1}\right.$, AkzoNobel, $\left.98 \%\right)$ was recrystallized from diethylether prior to use. ACTA (Figure 4-1 on page 28) was synthesized in a two-step procedure from $4,4^{\prime}$-azobis(4-cyanovaleric acid) by literature procedures ${ }^{[35,205]}$ The purity of ACTA was $\geq 97 \%$ determined by NMR analysis. 4,4'-Azobis(4-cyanovaleric acid $)\left(M=280.28 \mathrm{~g} \cdot \mathrm{mol}^{-1}\right.$, Fluka, $\left.\geq 99 \%\right)$ was dried at $25{ }^{\circ} \mathrm{C}$ under reduced 
pressure to remove residual water prior to synthesis. AIBN and ACTA were stored at $3{ }^{\circ} \mathrm{C}$.

\subsubsection{Miscellaneous Substances}

\section{Solvents}

Toluene, methanol, and dichloromethane were used as received. For SEC analysis, THF (Roth, $\geq 95 \%$, stabilized with 2,6-di-tert-butyl-4-methylphenol) and DMAc (Aldrich, CHROMASOLV ${ }^{\circledR}$ Plus, $\geq 99.9 \%$ ) were used, respectively.

\section{Fumed Silica}

The fumed silica particles $\left(M=60.08 \mathrm{~g} \cdot \mathrm{mol}^{-1}\right.$, Aldrich) with $7 \mathrm{~nm}$ of nominal particle size, a surface area of $390 \mathrm{~m}^{2} \cdot \mathrm{g}^{-1}$, and a bulk density of $36.8 \mathrm{~g} \cdot \mathrm{L}^{-1}$ were used as received. Particle sizes are given for primary particles, which form branched, chain-like aggregates of some tenths of micrometers.

Unless otherwise noted, all other chemicals were purchased (Aldrich, Fluka, Merck, ABCR, Roth) and used without further purification.

\subsubsection{RAFT Agents}

\section{Cumyl Dithiobenzoate (CDB)}

CDB (Scheme 10-1) was synthesized by a literature procedure. ${ }^{[206]}$ The purity was $\geq 98 \%$ according to NMR spectroscopy.<smiles>CC(C)(SC(=S)c1ccccc1)c1ccccc1</smiles>

Scheme 10-1. Chemical structure of CDB.

\section{Propyl-((trimethoxysilyl)ethyl-phenylmethyl)trithiocarbonate (PTPT)}

To a solution of sodium methoxide ( $10.8 \mathrm{~g}, 50 \mathrm{mmol}, 25 \mathrm{wt} .-\%$ in methanol) in methanol $(20 \mathrm{~mL})$, 1-propanethiol $(4.53 \mathrm{~mL}, 3.81 \mathrm{~g}, 50 \mathrm{mmol})$ was added dropwise at RT. The mixture was stirred for 1 hour and carbon disulfide ( $4.01 \mathrm{~mL}, 5.08 \mathrm{~g}, 66.7 \mathrm{mmol}$ ) was added dropwise to the solution. After 5 hours ((chloromethyl)phenylethyl)trimethoxysilane $(12.3 \mathrm{~mL}, 13.7 \mathrm{~g}, 50 \mathrm{mmol}$, mixture 
of meta- and para-isomers) was added and the mixture was stirred for 16 hours. The solvent was evaporated under reduced pressure and the residuals were removed via dissolving in dichloromethane and subsequent filtration. After removal of the dichloromethane under reduced pressure, the product PTPT (Scheme 6-1 on page 73) was obtained as yellowish oil $(12.3 \mathrm{~g}, 31.5 \mathrm{mmol}$, $63.0 \%)$.

${ }^{1} \mathrm{H}-\mathrm{NMR}\left(300 \mathrm{MHz}, \mathrm{CDCl}_{3}\right): \delta=0.90-1.05\left(\mathrm{~m}, 5 \mathrm{H}, \mathrm{Si}-\mathrm{CH}_{2}\right.$ and $\mathrm{S}-\mathrm{CH}_{2}-\mathrm{CH}_{2}-$ $\left.\mathrm{CH}_{3}\right), 1.65-1.80\left(\mathrm{~m}, 2 \mathrm{H}, \mathrm{S}-\mathrm{CH}_{2}-\mathrm{CH}_{2}-\mathrm{CH}_{3}\right), 2.60-2.78\left(\mathrm{~m}, 2 \mathrm{H}, \mathrm{Si}-\mathrm{CH}_{2}-\mathrm{CH}_{2}\right)$, 3.28-3.38 (m, $\left.2 \mathrm{H}, \mathrm{S}-\mathrm{CH}_{2}-\mathrm{CH}_{2}-\mathrm{CH}_{3}\right), 3.54\left(\mathrm{~s}, 9 \mathrm{H}, 3 \times \mathrm{Si}-\mathrm{O}-\mathrm{CH}_{3}\right), \quad 4.50-4.60$ $\left(2 \times \mathrm{s}, 2 \mathrm{H}, \mathrm{S}-\mathrm{CH}_{2}-\mathrm{C}_{6} \mathrm{H}_{4}\right.$ of meta- and para-isomers $), 7.04-7.30\left(\mathrm{~m}, 4 \mathrm{H}, \mathrm{C}_{6} \mathrm{H}_{4}\right)$ ppm.

${ }^{13} \mathrm{C}-\mathrm{NMR}\left(75 \mathrm{MHz}, \mathrm{CDCl}_{3}\right): \delta=11.1\left(\mathrm{Si}-\mathrm{CH}_{2}\right), 13.4\left(\mathrm{~S}-\mathrm{CH}_{2}-\mathrm{CH}_{2}-\mathrm{CH}_{3}\right), 21.5(\mathrm{~S}-$ $\mathrm{CH}_{2}-\mathrm{CH}_{2}-\mathrm{CH}_{3}$ ), 28.3, 28.5 ( $\mathrm{Si}-\mathrm{CH}_{2}-\mathrm{CH}_{2}$ of meta- and para-isomers), 38.8 (S$\mathrm{CH}_{2}-\mathrm{CH}_{2}-\mathrm{CH}_{3}$ ), 41.2, 41.5 ( $\mathrm{S}-\mathrm{CH}_{2}-\mathrm{C}_{6} \mathrm{H}_{4}$ of meta- and para-isomers), 50.5 ( $\mathrm{Si}-\mathrm{O}-$ $\left.\mathrm{CH}_{3}\right), 126.6,127.2,128.1,128.65,128.70,129.2,132.2,134.9,143.9,144.9\left(C_{6} \mathrm{H}_{4}\right.$ of meta- and para-isomers), $223.8(C=\mathrm{S}) \mathrm{ppm}$.

\section{Hexyl(phenylethyl)trithiocarbonate (HPT)}

HPT (Scheme 6-1 on page 73) was synthesized as described in the literature. ${ }^{[207]}$ The purity was $\geq 97 \%$ according to NMR spectroscopy.

\section{Dimethyl-2,6-di(propyltrithiocarbonyl)heptanedioate (DPTH)}

To a solution of sodium methoxide $(9.0 \mathrm{~g}, 50 \mathrm{mmol}, 30 \mathrm{wt} .-\%$ in methanol) in methanol $(20 \mathrm{~mL})$, 1-propanethiol $(4.53 \mathrm{~mL}, 3.81 \mathrm{~g}, 50 \mathrm{mmol})$ was added dropwise at RT. The mixture was stirred for 1 hour and carbon disulfide (4.01 mL, $5.08 \mathrm{~g}, 66.7 \mathrm{mmol}$ ) was added dropwise to the solution. After 5 hours dimethyl-2,6-dibromoheptanedioate $(5.44 \mathrm{~mL}, 8.65 \mathrm{~g}, 25 \mathrm{mmol})$ was added and the mixture was stirred for 16 hours. The solvent was evaporated under reduced pressure and the residuals were removed via dissolving in dichloromethane and subsequent filtration. After removal of the dichloromethane under reduced pressure, the product DPTH (Scheme 7-1 on page 88) was obtained as yellowish oil (10.2 g, $20.9 \mathrm{mmol}, 83.6 \%)$.

${ }^{1} \mathrm{H}-\mathrm{NMR}\left(300 \mathrm{MHz}, \mathrm{CDCl}_{3}\right): \delta=0.99\left(\mathrm{t}, 6 \mathrm{H}, \mathcal{f}=7.5 \mathrm{~Hz}, 2 \times \mathrm{S}-\mathrm{CH}_{2}-\mathrm{CH}_{2}-\mathrm{CH}_{3}\right)$, 1.3-1.6 (m, $\left.2 \mathrm{H}, \mathrm{S}-\mathrm{CH}-\mathrm{CH}_{2}-\mathrm{CH}_{2}-\mathrm{CH}_{2}-\mathrm{CH}-\mathrm{S}\right), 1.6-1.8\left(\mathrm{~m}, 4 \mathrm{H}, 2 \times \mathrm{S}^{-} \mathrm{CH}_{2}-\mathrm{CH}_{2}-\right.$ $\left.\mathrm{CH}_{3}\right), 1.8-2.1\left(\mathrm{~m}, 4 \mathrm{H}, \mathrm{S}-\mathrm{CH}-\mathrm{CH}_{2}-\mathrm{CH}_{2}-\mathrm{CH}_{2}-\mathrm{CH}-\mathrm{S}\right), 3.31(\mathrm{t}, 4 \mathrm{H}, \mathcal{J}=7.5 \mathrm{~Hz}$, $\left.2 \times \mathrm{S}-\mathrm{CH}_{2}-\mathrm{CH}_{2}-\mathrm{CH}_{3}\right), 3.70\left(\mathrm{~s}, 6 \mathrm{H}, 2 \times \mathrm{O}-\mathrm{CH}_{3}\right), 4.80(\mathrm{t}, 2 \mathrm{H}, \mathrm{J}=6 \mathrm{~Hz}, \mathrm{~S}-\mathrm{CH}-$ $\left.\mathrm{CH}_{2}-\mathrm{CH}_{2}-\mathrm{CH}_{2}-\mathrm{CH}-\mathrm{S}\right) \mathrm{ppm}$. 
${ }^{13} \mathrm{C}-\mathrm{NMR}\left(75 \mathrm{MHz}, \mathrm{CDCl}_{3}\right): \delta=13.7\left(\mathrm{~S}-\mathrm{CH}_{2}-\mathrm{CH}_{2}-\mathrm{CH}_{3}\right), 21.7\left(\mathrm{~S}-\mathrm{CH}-\mathrm{CH}_{2}-\mathrm{CH}_{2}-\right.$ $\left.\mathrm{CH}_{2}-\mathrm{CH}-\mathrm{S}\right), 24.7\left(\mathrm{~S}-\mathrm{CH}_{2}-\mathrm{CH}_{2}-\mathrm{CH}_{3}\right), 31.1\left(\mathrm{~S}-\mathrm{CH}-\mathrm{CH}_{2}-\mathrm{CH}_{2}-\mathrm{CH}_{2}-\mathrm{CH}-\mathrm{S}\right), 39.4$ $\left(\mathrm{S}-\mathrm{CH}_{2}-\mathrm{CH}_{2}-\mathrm{CH}_{3}\right), 52.5\left(\mathrm{O}-\mathrm{CH}_{3}\right), 53.1\left(\mathrm{~S}-\mathrm{CH}-\mathrm{CH}_{2}-\mathrm{CH}_{2}-\mathrm{CH}_{2}-\mathrm{CH}-\mathrm{S}\right), 171.1$ $(C=\mathrm{O}), 222.1(C=\mathrm{S})$.

\subsection{Experimental Procedures}

\subsubsection{Immobilization of ACTA}

To a vigorously stirred suspension of silica nanoparticles $(10 \mathrm{~g})$ in dichloromethane $(300 \mathrm{~mL})$ ACTA $(0.32 \mathrm{~g}, 0.46 \mathrm{mmol})$ was added dropwise as a solution in dichloromethane $(20 \mathrm{~mL})$. A few drops of a saturated solution of maleic anyhdride in water were added and the mixture was stirred at RT for $3 \mathrm{~h}$. The solvent was removed in vacuo and the crude product was washed with dichloromethane in a Soxhlet apparatus for $12 \mathrm{~h}$ to remove the residuals. The grafting densities of ACTA on the nanoparticle surface were determined by elemental analysis.

\subsubsection{Immobilization of PTPT}

To a vigorously stirred suspension of silica nanoparticles $(4 \mathrm{~g})$ in toluene $(200 \mathrm{~mL})$ PTPT $(0.46 \mathrm{~g}, 1.2 \mathrm{mmol})$ was added dropwise as a solution in toluene $(10 \mathrm{~mL})$ under vigorous stirring. A few drops of a saturated solution of maleic anyhdride in water were added and the mixture was stirred at RT for $3 \mathrm{~h}$. The solvent was removed in vacuo and the crude product was washed with dichloromethane in a Soxhlet apparatus for $12 \mathrm{~h}$ to remove the residuals. The grafting density of PTPT on the nanoparticle surface was determined by elemental analysis.

\subsubsection{Pulsed-Laser Polymerizations (PLP)}

The PLP experiments were carried out at ambient pressure and temperatures of $5{ }^{\circ} \mathrm{C}$ and $25^{\circ} \mathrm{C}$ in the case of $n \mathrm{BA}$ and $25^{\circ} \mathrm{C}$ and $26{ }^{\circ} \mathrm{C}$ in the case of styrene. The $\mathrm{XeF}$ line $(351 \mathrm{~nm})$ of an LPX 200 excimer laser was used to trigger the initiation and thus polymerization. The laser repetition rates used were $100 \mathrm{~Hz}$ for $n \mathrm{BA}$ and $5 \mathrm{~Hz}$ for styrene. The incident laser energy was between 30 and $130 \mathrm{~mJ}$ per pulse. Overall monomer conversions reached maximum values of $4 \%$ and were determined by gravimetry. All samples were deoxygenated via flushing with nitrogen for 15 minutes. For the polymerizations with AIBN and DMPA, 
initiator concentrations of $1.98-2.25 \mathrm{mmol} \cdot \mathrm{L}^{-1}$ were used. In case of the surfaceinitiated polymerizations, $0.2 \mathrm{~g}$ of modified silica per $1 \mathrm{~mL}$ of monomer solution were used, which was sufficient to prevent the precipitation of the silica particles and hence lead to an even distribution of the modified particles in the monomer solution. The amount of modified silica used resulted in initiator concentrations of $8.8-22 \mathrm{mmol} \cdot \mathrm{L}^{-1}$ depending on the loading of initiator. After polymerization the reaction mixtures were poured into glass vials and residual monomer was removed in vacuo. If present, the polymer in solution was subjected to SEC analysis and subsequently removed from the polymer-silica hybrid by washing with THF. The residual solid was then treated with hydrofluoric acid to isolate and analyze the surface-bound polymer. ${ }^{[39]}$

\subsubsection{Surface-Initiated Conventional Radical Polymerization of Styrene}

A solution of styrene ( $25 \mathrm{~g})$ was thoroughly degassed via three freeze-pumpthaw cycles. The ACTA-modified silica ( 0.069 molecules $\cdot \mathrm{nm}^{-2}$ according to elemental analysis) was weighed in glass vials $(0.5 \mathrm{~g}$ each) that were each equipped with a magnetic stir bar. The monomer $(4 \mathrm{~mL})$ was mixed with the silica in an argon-filled glove box. The vials were sealed with Teflon septa and subsequently heated to $60{ }^{\circ} \mathrm{C}$. The polymerizations were stopped by cooling the reaction mixtures in ice water after distinct time periods. After evaporation of the monomer, the polymer formed in solution was isolated by suspending a small fraction of the solid mixture in THF. The suspension was filtered and the obtained solution was subjected to SEC analysis. The remaining solid was washed with dichloromethane in a Soxhlet apparatus for $12 \mathrm{~h}$ to remove residuals from the solution phase and to isolate the surface-bound polymer.

\subsubsection{Surface-Initiated RAFT Polymerization of Styrene}

A solution of $\mathrm{CDB}(73.5 \mathrm{mg}, 0.27 \mathrm{mmol})$ in styrene $(25.7 \mathrm{~g})$ was thoroughly degassed via three freeze-pump-thaw cycles. The ACTA-modified silica (0.061 molecules $\cdot \mathrm{nm}^{-2}$ according to elemental analysis) was weighed in glass vials $(0.4 \mathrm{~g}$ each) that were each equipped with a magnetic stir bar. The monomer $(4 \mathrm{~mL})$ was mixed with the silica in an argon-filled glove box. The vials were sealed with Teflon septa and subsequently heated to $60{ }^{\circ} \mathrm{C}$. The polymerizations were stopped by cooling the reaction mixtures in ice water after distinct time periods. After evaporation of the monomer, the polymer formed in solution was isolated by suspending a small fraction of the solid mixture in THF. The suspension was filtered and the obtained solution was 
subjected to SEC analysis. The remaining solid was washed with dichloromethane in a Soxhlet apparatus for $12 \mathrm{~h}$ to remove residuals from the solution phase and to isolate the surface-bound polymer.

\subsubsection{Cleavage of Silica-Bound Polymer}

The surface-bound polymer was detached by treatment of the silica-polymer hybrids with hydrofluoric acid. In a typical run, the silica-polymer hybrid $(100 \mathrm{mg})$ was suspended in THF $(5 \mathrm{~mL})$ and a $40 \mathrm{wt} . \%$ aqueous solution of hydrogen fluoride $(0.5 \mathrm{~mL})$ was added. The mixture was stirred at RT for $3 \mathrm{~h}$ and poured into an aluminum dish, in which the volatiles were allowed to evaporate for at least two days. The recovered solid was dissolved in THF and analyzed by SEC.

\subsubsection{Synthesis of Silica-Filled Styrene-nBA Copolymers}

In a typical run, a solution of AIBN ( $57 \mathrm{mg}, 0.35 \mathrm{mmol})$, respective silica particles $(0.2-0.5 \mathrm{~g})$, and respective RAFT agent $\left(4.5 \mathrm{mmol} \cdot \mathrm{L}^{-1}\right)$ in styrene $(8 \mathrm{~g})$, $n \mathrm{BA}(9.85 \mathrm{~g})$, and toluene $(25 \mathrm{~g})$ was thoroughly degassed via three freezepump-thaw cycles. A magnetic stir bar was added to the suspension and the reaction mixture was subsequently heated to $60{ }^{\circ} \mathrm{C}$. The polymerization was stopped by removing the oil bath after approximately $40 \mathrm{~h}$. The polymerization solution was poured into a Teflon bowl for drying and characterizing the obtained polymer.

\subsubsection{Extraction of Pure Styrene-nBA Copolymers}

After tensile analysis, the selected silica-filled copolymers were washed with toluene in a Soxhlet apparatus for three days. Afterwards, the filtrate solution was poured into a Teflon bowl, from which the tensile specimens were prepared.

\subsubsection{Synthesis of ABA Type $t \mathrm{BA}-\mathrm{AA}$ Copolymers}

In a typical experiment, a solution of AIBN $(27.1 \mathrm{mg}, 0.165 \mathrm{mmol})$ and DPTH (677 mg, $1.39 \mathrm{mmol})$ in $t \mathrm{BA}(15 \mathrm{~g}, 0.117 \mathrm{~mol})$ and toluene $(15 \mathrm{~g})$ was degassed via three freeze-pump-thaw cycles and channelled in an argon-filled glove box. The mixture was then evenly portioned to glass vials that were sealed with 
Teflon septa and subsequently heated to $60{ }^{\circ} \mathrm{C}$ for distinct time periods. Polymerizations were stopped by cooling the vials in ice water. Total monomer conversions and MMDs were determined by gravimetry and SEC, respectively. Afterwards, the poly( $t \mathrm{BA})$ samples were used as macromolecular RAFT agents for extension polymerizations to yield the ABA type copolymers. The corresponding polymerization procedure was similar to the one for the inner blocks described above. A typical reaction mixture consisted of AIBN (3.0 mg, $0.02 \mathrm{mmol}$ ) together with the respective macromolecular RAFT agent from the first step, $t \mathrm{BA}(8.0 \mathrm{~g}, 62.4 \mathrm{mmol})$, AA $(0.4 \mathrm{~g}, 5.6 \mathrm{mmol})$, and toluene $(8.0 \mathrm{~g})$.

\subsubsection{Synthesis of Random MA-AA Copolymers}

In a typical run, a solution of AIBN (41 mg, $0.25 \mathrm{mmol}$ ) and DPTH (242 mg, $0.50 \mathrm{mmol})$ in MA $(20 \mathrm{~g})$, AA (2 g) and toluene $(25 \mathrm{~g})$ was thoroughly degassed via three freeze-pump-thaw cycles. A magnetic stir bar was added to the solution and the reaction mixture was subsequently heated to $60{ }^{\circ} \mathrm{C}$. The polymerization was stopped by removing the oil bath after approximately $4 \mathrm{~h}$. The polymerization solution was poured into a Teflon bowl for drying and characterizing the obtained polymer.

\subsubsection{Synthesis of the ABA Type MA-AA Copolymer}

A solution of AIBN (75 mg, $0.46 \mathrm{mmol})$ and DPTH $(535 \mathrm{mg}, 1.1 \mathrm{mmol})$ in MA $(27.7 \mathrm{~g})$ and toluene $(25 \mathrm{~g})$ was thoroughly degassed via three freeze-pumpthaw cycles. A magnetic stir bar was added to the solution and the reaction mixture was subsequently heated to $60^{\circ} \mathrm{C}$. The polymerization was stopped by removing the oil bath after approximately $5 \mathrm{~h}$ and the polymer solution was poured in a Teflon bowl. The obtained poly(MA) was dried for two weeks at ambient pressure and for another two days in a vacuum oven at $60{ }^{\circ} \mathrm{C}$. Afterwards, it used as a macromolecular RAFT agent for the extension polymerization to yield the ABA type copolymer. The corresponding polymerization procedure was similar to the first step described above except the reaction time, which was $3 \mathrm{~h}$. The reaction mixture consisted of AIBN (20 $\mathrm{mg}, 0.12 \mathrm{mmol}$ ), the macromolecular RAFT agent (4.92 $\mathrm{g}, 0.21 \mathrm{mmol}), \mathrm{MA}$ $(10 \mathrm{~g}, 0.12 \mathrm{~mol})$, AA $(1 \mathrm{~g}, 14 \mathrm{mmol})$, and toluene $(14 \mathrm{~g})$. After the reaction, the polymerization solution was poured into a Teflon bowl for drying and characterizing the obtained polymer. 


\subsubsection{Sample Preparation for Tensile Analysis}

The tensile specimens were obtained by the solution-casting method. A solution of the polymer $(\sim 10 \mathrm{~g})$ in toluene $(\sim 100 \mathrm{~mL})$ was poured into a Teflon bowl and the solvent was allowed to evaporate over a period of at least 10 days. The polymer film was dried in a vacuum oven for two days at $23^{\circ} \mathrm{C}$ and subsequently annealed for $24 \mathrm{~h}$ at $120^{\circ} \mathrm{C}$ under vacuum. Afterwards, the film was allowed to cool to RT and separated from the Teflon bowl. Samples with approximate dimensions of $50 \mathrm{~mm} \times 10 \mathrm{~mm} \times 1 \mathrm{~mm}$ were cut from the freestanding film.

\subsubsection{Sample Preparation for DMA}

After the polymer synthesis, the samples were pressed into a cylindrical form with a pressure of $1.25 \mathrm{kPa}$, which had a diameter of $6 \mathrm{~mm}$ and a height of 2$6 \mathrm{~mm}$. For this purpose, a self-designed holder of hardened steel was used. The polymer cylinders were then transferred into an aluminum sample container with a cap on top (Figure 10-1), which transferred the applied forces to the samples. The sample container had a diameter and a height of $8 \mathrm{~mm}$. The sample was placed in the center of the container to avoid any contact with the wall.

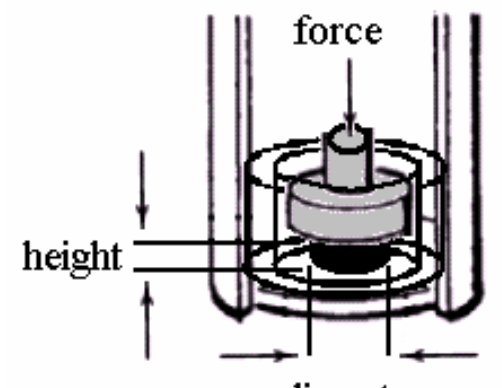

diameter

Figure 10-1. Aluminum container and cap for transferring the forces to the polymer samples in DMA.

\subsection{Instrumentation}

\subsubsection{Size-Exclusion Chromatography}

MMDs were determined by means of SEC. For the MA-AA copolymers covered in chapter 8, an SEC system that uses DMAc as the eluent was used. For all 
other polymers in this thesis, the MMDs were determined on an SEC setup that uses THF as the eluent.

\section{THF-SEC Setup}

The SEC unit contained an autosampler (JASCO AS-2055Plus), a highperformance liquid chromatography (HPLC) pump (Waters 515), a pre-column (Polymer Standards Service (PSS) SDV, $8 \times 50 \mathrm{~mm}$, particle size: $5 \mu \mathrm{m}$ ), three separation columns (PSS SDV, $8 \times 300 \mathrm{~mm}$, particles size: $5 \mu \mathrm{m}$, pore sizes: $10^{5}$, $10^{3}$ and $10^{2} \AA$ ), a refractive index detector (Waters 2410), and a UV detector (Viscothek VE 3210 at $330 \mathrm{~nm}$ ). The eluent THF was tempered at $35^{\circ} \mathrm{C}$ and had a flow rate of $1 \mathrm{~mL} \cdot \mathrm{min}^{-1}$. The polymers were dissolved in THF at a concentration of $3 \mathrm{mg} \cdot \mathrm{mL}^{-1}$ together with traces of toluene as the internal standard. Data acquisition and processing were carried out using the WinGPC 6.20 software from PSS. The SEC setup was calibrated against polystyrene standards of low polydispersity from PSS. MH parameters for poly $(n \mathrm{BA})$, poly $(t \mathrm{BA})$ and poly(MA) (Table 10-1) allowed access to absolute molar masses according to the principles of universal calibration. For the styrene- $n$ BA copolymers, the $M_{n}$ values obtained from the polystyrene and poly $(n \mathrm{BA})$ calibration were averaged. In case of the $t \mathrm{BA}-\mathrm{AA}$ copolymers, only the poly $(t \mathrm{BA})$ calibration was applied.

Table 10-1. MH parameters used for obtaining absolute molar masses of the polymers covered in this work.

\begin{tabular}{lrc}
\hline \multicolumn{1}{c|}{ Polymer } & $K\left[\mathrm{~mL} \cdot \mathrm{g}^{-1}\right]$ & \multicolumn{1}{c}{$a$} \\
\hline polystyrene ${ }^{[208]}$ & $1.41 \cdot 10^{-2}$ & 0.700 \\
$\operatorname{poly}(n \mathrm{BA})^{[153]}$ & $1.22 \cdot 10^{-2}$ & 0.700 \\
$\operatorname{poly}(t \mathrm{BA})^{[209]}$ & $4.34 \cdot 10^{-2}$ & 0.600 \\
$\operatorname{poly}(\mathrm{MA})^{[81]}$ & $1.68 \cdot 10^{-2}$ & 0.696 \\
\hline
\end{tabular}

\section{DMAc-SEC Setup}

The Agilent 1260 Infinity SEC unit contained an autosampler, an HPLC pump, a pre-column (PSS SDV, $8 \times 50 \mathrm{~mm}$, particle size: $10 \mu \mathrm{m}$ ), three separation columns (PSS SDV, $8 \times 300 \mathrm{~mm}$, particle size: $10 \mu \mathrm{m}$, pore sizes: $10^{3}, 10^{3}$ and $30 \AA$ ), a refractive index detector, and a UV detector (at $330 \mathrm{~nm}$ ). The eluent DMAc contained $0.1 \mathrm{wt} . \%$ lithium bromide and had a flow rate of $0.8 \mathrm{~mL} \cdot \mathrm{min}^{-1}$ at a temperature of $30^{\circ} \mathrm{C}$. The polymers were dissolved in DMAc at a concentration of $3 \mathrm{mg} \cdot \mathrm{mL}^{-1}$. Data acquisition and processing were carried out 
using the WinGPC Unity Build 9586 software from Polymer Standards Service (PSS). The SEC setup was calibrated against polystyrene standards of low polydispersity from PSS.

\subsubsection{NMR Spectroscopy}

NMR spectra were recorded on a Varian Unity 300 spectrometer at RT using $\mathrm{CDCl}_{3}$ as the solvent. The concentrations of the analytes were in the range of $30-60 \mathrm{~g} \cdot \mathrm{L}^{-1}$. The residual proton signal of the deuterated solvent served as internal standard for the ${ }^{1} \mathrm{H}-\mathrm{NMR}$ spectra. The NMR data were processed with the program MestReNova 6.2.1-7569 (MESTRELAB RESEARCH).

\subsubsection{Elemental Analysis}

All elemental analyses were performed at the analytical laboratory of the Institute of Inorganic Chemistry in Göttingen.

Elemental analyses for carbon, hydrogen, nitrogen and sulfur $(\mathrm{C}, \mathrm{H}, \mathrm{N}, \mathrm{S})$ were carried out on a Elementar Vario El III elemental analyzer.

In case of the elemental analyses for silicon, the compound was ionized and subsequently subjected to UV/Vis analysis on a PerkinElmer Lambda 2 spectrometer.

\subsubsection{Thermogravimetric Analysis}

TGA was carried out using a Netzsch STA 409 PC thermogravimetric analyzer from $\mathrm{RT}$ to $600{ }^{\circ} \mathrm{C}$ at a rate of $10 \mathrm{~K} \cdot \mathrm{min}^{-1}$ under a nitrogen atmosphere.

\subsubsection{Scanning Electron Microscopy}

SE micrographs were taken on a LEO SUPRA 35 electron microscope operated at $15 \mathrm{kV}$. The pressure inside the microscope was approximately $5 \cdot 10^{-7} \mathrm{mbar}$. Prior to analysis, the silica-polymer hybrids were coated with a gold layer with a thickness of a few nanometers to assure the discharges of the probes during imaging.

\subsubsection{Tensile Testing}

Tensile measurements were performed on a Zwicki $2.5 \mathrm{kN}$ Allround testing machine (Zwick Roell) at $23^{\circ} \mathrm{C}$ and ambient pressure. Specimens were drawn at 
a speed of $50 \mathrm{~mm} \cdot \mathrm{min}^{-1}$ with an initial sample-grip distance of $15 \mathrm{~mm}$. The apparent stress was recorded against the elongation. The stress-strain data were processed with the program testXpert ${ }^{\circledR}$ II, version 3.0 (Zwick Roell).

\subsubsection{Dynamic Mechanical Analysis}

The relaxation spectra were measured with a DMA 7 from PerkinElmer with a static force of $500 \mathrm{mN}$, a dynamic force of $400 \mathrm{mN}$, a frequency of $5 \mathrm{~Hz}$ and a heating rate of $1 \mathrm{~K} \cdot \mathrm{min}^{-1}$. The sample height and the phase angle were detected via a linear variable differential transformer (LVDT). For determination of the glass transition temperature, the onset of the sample height decrease in the DMA measurements was used.

\subsection{Simulations}

The kinetic models of the surface-initiated RAFT polymerization, outlined in subchapter 5.2.4 and illustrated in appendix A, were implemented into the program package PREDICI ${ }^{\circledR}$, version 6.4.8. The input data consist of the kinetic parameters, the individual reaction steps, the initial values for the reaction components and the reactor variables. The resulting system of differential equations is integrated for a given reaction time by PREDICI ${ }^{\circledR}$. Among other things, this program allows for the simulation of MMDs of macromolecules that result from a polymerization reaction as well as the corresponding mean values and the concentration profiles of the reagents. 



\section{References}

[1] L. H. Baekeland, US Patent 942 699, 1907.

[2] H. Staudinger, J. Fritschi, Helv. Chim. Acta 1922, 5, 785-806.

[3] K. Matyjaszewski, Y. Gnanou, L. Leibler, Macromolecular Egineering, Wiley-VCH Verlag GmbH \& Co. KGaA, Weinheim, 2007.

[4] Gesamtverband Kunststoffverarbeitende Industrie e.V. (GKV), $w w w . g k v . d e(26.05 .2011)$.

[5] G. Moad, E. Rizzardo, S. H. Thang, Accounts of Chemical Research 2008, $41,1133-1142$.

[6] J. Chiefari, Y. K. Chong, F. Ercole, J. Krstina, J. Jeffery, T. P. T. Le, R. T. A. Mayadunne, G. F. Meijs, C. L. Moad, G. Moad, E. Rizzardo, S. H. Thang, Macromolecules 1998, 31, 5559-5562.

[7] M. Buback, H. Lendle, Macromol. Chem. Phys. 1983, 184, 193-206.

[8] M. Buback, Macromol. Chem. Phys. 1990, 191, 1575-1587.

[9] T. von Werne, T. E. Patten, J. Am. Chem. Soc. 1999, 121, 7409-7410.

[10] Y. K. Huang, Q. Liu, X. D. Zhou, S. Perrier, Y. L. Zhao, Macromolecules 2009, 42, 5509-5517.

[11] A. Schmid, S. P. Armes, C. A. P. Leite, F. Galembeck, Langmuir 2009, 25, 2486-2494.

[12] Q. Yang, L. Wang, W. Xiang, J. Zhou, Q. h. Tan, Polymer 2007, 48, 34443451. 
[13] L. Quaroni, G. Chumanov, f. Am. Chem. Soc. 1999, 121, 10642-10643.

[14] K. Ohno, K. Koh, Y. Tsujii, T. Fukuda, Angew. Chem. Int. Ed. 2003, 42, 2751-2754.

[15] J. Raula, J. Shan, M. Nuopponen, A. Niskanen, H. Jiang, E. I. Kauppinen, H. Tenhu, Langmuir 2003, 19, 3499-3504.

[16] D.-H. Han, C.-Y. Pan, J. Polym. Sci. Part A: Polym. Chem. 2008, 46, 341352.

[17] D. Li, Y. Luo, B.-G. Li, S. Zhu, J. Polym. Sci. Part A: Polym. Chem. 2008, 46, 970-978.

[18] E. Turan, S. Demirci, T. Caykara, Thin Solid Films 2010, 518, 5950-5954.

[19] M. Baum, W. J. Brittain, Macromolecules 2002, 35, 610-615.

[20] M. D. Rowe-Konopacki, S. G. Boyes, Macromolecules 2007, 40, 879-888.

[21] J. H. Jeon, J. H. Lim, K. M. Kim, Polymer 2009, 50, 4488-4495.

[22] P. Liu, f. Nanopart. Res. 2009, 11, 1011-1016.

[23] J. Ryu, B. Ramaraj, K. R. Yoon, Surf. Interface Anal. 2009, 41, 303-309.

[24] J. W. Hotchkiss, A. B. Lowe, S. G. Boyes, Chem. Mater. 2007, 19, 6-13.

[25] D. Roy, J. T. Guthrie, S. Perrier, Macromolecules 2005, 38, 10363-10372.

[26] M. Barsbay, O. Güven, M. H. Stenzel, T. P. Davis, C. Barner-Kowollik, L. Barner, Macromolecules 2007, 40, 7140-7147.

[27] C. Yoshikawa, A. Goto, Y. Tsujii, T. Fukuda, K. Yamamoto, A. Kishida, Macromolecules 2005, 38, 4604-4610.

[28] L. Barner, S. Perera, S. Sandanayake, T. P. Davis, f. Polym. Sci. Part A: Polym. Chem. 2006, 44, 857-864.

[29] K. Kiani, D. J. T. Hill, F. Rasoul, M. Whittaker, L. Rintoul, f. Polym. Sci. Part A: Polym. Chem. 2007, 45, 1074-1083.

[30] A. C. Costa, R. J. Composto, P. Vlcek, Macromolecules 2003, 36, 3254-3260. 
[31] H. W. Ma, J. H. Hyun, P. Stiller, A. Chilkoti, Adv. Mater. 2004, 16, 338341.

[32] G. Carrot, S. M. Scholz, C. J. G. Plummer, J. G. Hilborn, J. L. Hedrick, Chem. Mater. 1999, 11, 3571-3577.

[33] O. Prucker, J. Rühe, Macromolecules 1998, 31, 602-613.

[34] O. Prucker, J. Rühe, Macromolecules 1998, 31, 592-601.

[35] R. Rotzoll, P. Vana, Aust. f. Chem. 2009, 62, 1473-1478.

[36] P. Pasetto, H. Blas, F. Audouin, C. Boissiere, C. Sanchez, M. Save, B. Charleux, Macromolecules 2009, 42, 5983-5995.

[37] M. Wulkow, Macromol. Theory Simul. 1996, 5, 393-416.

[38] P. Vana, A. Goto, Macromol. Theory Simul. 2010, 19, 24-35.

[39] R. Rotzoll, P. Vana, f. Polym. Sci. Part A: Polym. Chem. 2008, 46, 76567666.

[40] H. Zou, S. Wu, J. Shen, Chem. Rev. 2008, 108, 3893-3957.

[41] H.-G. Elias, An Introduction to Plastics, Wiley-VCH GmbH \& Co. KGaA, Weinheim, 2003.

[42] S. Perrier, P. Takolpuckdee, f. Polym. Sci. Part A: Polym. Chem. 2005, 43, 5347-5393.

[43] J. Hachenberg, B. Steisel, U. Nergui, D. Bedorf, M. Buback, K. Samwer, Int. F. Mat. Res. 2008, 99, 502-506.

[44] D. S. Jones, Int. F. Pharm. 1999, 179, 167-178.

[45] K. P. Menard, Dynamic Mechanical Analysis - A Practical Introduction, CRC Press, New York, 1999.

[46] J. D. Ferry, Viscoelastic Properties of Polymers, John Wiley \& Sons, New York, 1980.

[47] L. H. Sperling, Introduction to Physical Polymer Science, WileyInterscience, New Jersey, 2006. 
[48] P. Rösner, K. Samwer, R. O. Pohl, S. Schneider, Rev. Sci. Instrum. 2003, 74, 3395-3399.

[49] P. Lunkenheimer, U. Schneider, R. Brand, A. Loid, Contemp. Phys. 2000, 41, 15-36.

[50] J. Hachenberg, K. Samwer, f. Non-Cryst. Solids 2006, 352, 5110-5113.

[51] P. Rösner, K. Samwer, P. Lunkenheimer, Europhys. Lett. 2004, 68, 226-232.

[52] H. Oberst, W. Retting, F. Macromol. Sci. Part B: Phys. 1971, 5, 559-590.

[53] T. Junkers, Dissertation, Göttingen University, 2006.

[54] P. Hesse, Dissertation, Göttingen University, 2008.

[55] A. D. Jenkins, R. G. Jones, G. Moad, Pure Appl. Chem. 2010, 82, 483-491.

[56] A. H. E. Müller, K. Matyjaszewski, Controlled and Living Polymerizations: From Mechanisms to Applications, Wiley-VCH, Weinheim, 2009.

[57] R. A. Hutchinson, M. T. Aronson, J. R. Richards, Macromolecules 1993, 26, 6410-6415.

[58] Z. Grubisic, P. Rempp, H. Benoit, f. Polym. Sci. Part B: Polym. Lett. 1967, 5, 753-759.

[59] S. F. Sun, Physical Chemistry of Macromolecules - Basic Principles and Issues, John Wiley \& Sons, Inc., Hoboken, 2004.

[60] H. Mark, Der feste Körper, Hirzel, Leipzig, 1938.

[61] R. Houwink, f. Prakt. Chem. 1940, 157, 15-18.

[62] W. F. Reed, Macromol. Chem. Phys. 1995, 196, 1539-1575.

[63] P. J. Wyatt, Anal. Chim. Acta 1993, 272, 1-40.

[64] O. F. Olaj, I. Bitai, F. Hinkelmann, Makromol. Chem. 1987, 188, 1689-1702.

[65] O. F. Olaj, I. Schnöll-Bitai, Eur. Polym. F. 1989, 25, 635-641. 
[66] G. Zifferer, A. Kornherr, I. Schnöll-Bitai, O. F. Olaj, Macromol. Symp. 2004, 217, 289-294.

[67] M. Buback, R. G. Gilbert, R. A. Hutchinson, B. Klumperman, F.-D. Kuchta, B. G. Manders, K. F. O'Driscoll, G. T. Russell, J. Schweer, Macromol. Chem. Phys. 1995, 196, 3267-3280.

[68] G. Moad, E. Rizzardo, S. H. Thang, Aust. f. Chem. 2005, 58, 379-410.

[69] C. J. Hawker, A. W. Bosman, E. Harth, Chem. Rev. 2001, 101, 3661-3688.

[70] K. Matyjaszewski, J. Xia, Chem. Rev. 2001, 101, 2921-2990.

[71] J.-S. Wang, K. Matyjaszewski, f. Am. Chem. Soc. 1995, 117, 5614-5615.

[72] M. Kamigaito, T. Ando, M. Sawamoto, Chemical Reviews 2001, 101, 36893745.

[73] R. T. A. Mayadunne, E. Rizzardo, J. Chiefari, Y. K. Chong, G. Moad, S. H. Thang, Macromolecules 1999, 32, 6977-6980.

[74] E. Rizzardo, J. Chiefari, B. Y. K. Chong, F. Ercole, J. Krstina, J. Jeffery, T. P. T. Le, R. T. A. Mayadunne, G. F. Meijs, C. L. Moad, G. Moad, S. H. Thang, Macromol. Symp. 1999, 143, 291-307.

[75] C. Barner-Kowollik, T. P. Davis, J. P. A. Heuts, M. H. Stenzel, P. Vana, M. Whittaker, f. Polym. Sci. Part A: Polym. Chem. 2003, 41, 365-375.

[76] C. Barner-Kowollik, S. Perrier, f. Polym. Sci. Part A: Polym. Chem. 2008, $46,5715-5723$.

[77] G. Moad, E. Rizzardo, S. H. Thang, Aust. f. Chem. 2006, 59, 669-692.

[78] G. Moad, E. Rizzardo, S. H. Thang, Aust. f. Chem. 2009, 62, 1402-1472.

[79] L. Barner, T. P. Davis, M. H. Stenzel, C. Barner-Kowollik, Macromol. Rapid Commun. 2007, 28, 539-559.

[80] J. F. Quinn, R. P. Chaplin, T. P. Davis, F. Polym. Sci. Part A: Polym. Chem. 2002, 40, 2956-2966.

[81] T. Arita, M. Buback, P. Vana, Macromolecules 2005, 38, 7935-7943. 
[82] Z. Szablan, A. A. Toy, T. P. Davis, X. J. Hao, M. H. Stenzel, C. BarnerKowollik, J. Polym. Sci. Part A: Polym. Chem. 2004, 42, 2432-2443.

[83] J. Rzayev, J. Penelle, Macromolecules 2002, 35, 1489-1490.

[84] G. Moad, E. Rizzardo, S. H. Thang, Polymer 2008, 49, 1079-1131.

[85] H. Shinoda, K. Matyjaszewski, L. Okrasa, M. Mierzwa, T. Pakula, Macromolecules 2003, 36, 4772-4778.

[86] J. Chiefari, R. T. A. Mayadunne, C. L. Moad, G. Moad, E. Rizzardo, A. Postma, M. A. Skidmore, S. H. Thang, Macromolecules 2003, 36, 22732283.

[87] Y. K. Chong, J. Krstina, T. P. T. Le, G. Moad, A. Postma, E. Rizzardo, S. H. Thang, Macromolecules 2003, 36, 2256-2272.

[88] G. Moad, Y. K. Chong, A. Postma, E. Rizzardo, S. H. Thang, Polymer 2005, 46, 8458-8468.

[89] M. Li, P. De, S. R. Gondi, B. S. Sumerlin, 7. Polym. Sci. Part A: Polym. Chem. 2008, 46, 5093-5100.

[90] A. J. Inglis, M. H. Stenzel, C. Barner-Kowollik, Macromol. Rapid Commun. 2009, 30, 1792-1798.

[91] M. Buback, P. Hesse, T. Junkers, P. Vana, Macromol. Rapid Commun. 2006, 27, 182-187.

[92] P. Vana, T. P. Davis, C. Barner-Kowollik, Macromol. Theory Simul. 2002, $11,823-835$.

[93] J. Barth, M. Buback, W. Meiser, P. Vana, Macromolecules 2010, 43, 51-54.

[94] C. Barner-Kowollik, M. Buback, B. Charleux, M. L. Coote, M. Drache, T. Fukuda, A. Goto, B. Klumperman, A. B. Lowe, J. B. McLeary, G. Moad, M. J. Monteiro, R. D. Sanderson, M. P. Tonge, P. Vana, F. Polym. Sci. Part A: Polym. Chem. 2006, 44, 5809-5831.

[95] B. Klumperman, E. T. A. van den Dungen, J. P. A. Heuts, M. J. Monteiro, Macromol. Rapid Commun. 2010, 31, 1846-1862.

[96] M. L. Coote, f. Phys. Chem. A 2005, 109, 1230-1239. 
[97] M. L. Coote, E. I. Izgorodina, E. H. Krenske, M. Busch, C. BarnerKowollik, Macromol. Rapid Commun. 2006, 27, 1015-1022.

[98] M. L. Coote, E. H. Krenske, E. I. Izgorodina, Macromol. Rapid Commun. 2006, 27, 473-497.

[99] E. I. Izgorodina, M. L. Coote, Macromol. Theory Simul. 2006, 15, 394-403.

[100] C. Y. Lin, M. L. Coote, Aust. f. Chem. 2009, 62, 1479-1483.

[101] C. Barner-Kowollik, J. F. Quinn, D. R. Morsley, T. P. Davis, f. Polym. Sci. Part A: Polym. Chem. 2001, 39, 1353-1365.

[102] A. Feldermann, M. L. Coote, M. H. Stenzel, T. P. Davis, C. BarnerKowollik, f. Am. Chem. Soc. 2004, 126, 15915-15923.

[103] M. L. Coote, Macromolecules 2004, 37, 5023-5031.

[104] Y. Kwak, A. Goto, Y. Tsujii, Y. Murata, K. Komatsu, T. Fukuda, Macromolecules 2002, 35, 3026-3029.

[105] A. R. Wang, S. P. Zhu, Y. W. Kwak, A. Goto, T. Fukuda, M. S. Monteiro, f. Polym. Sci. Part A: Polym. Chem. 2003, 41, 2833-2839.

[106] W. Meiser, J. Barth, M. Buback, H. Kattner, P. Vana, Macromolecules 2011, 44, 2474-2480.

[107] Y. Kwak, A. Goto, T. Fukuda, Macromolecules 2004, 37, 1219-1225.

[108] Y. Kwak, A. Goto, K. Komatsu, Y. Sugiura, T. Fukuda, Macromolecules 2004, 37, 4434-4440.

[109] D. Konkolewicz, B. S. Hawkett, A. Gray-Weale, S. Perrier, f. Polym. Sci. Part A: Polym. Chem. 2009, 47, 3455-3466.

[110] S. L. Brown, D. Konkolewicz, A. Gray-Weale, W. B. Motherwell, S. Perrier, Aust. f. Chem. 2009, 62, 1533-1536.

[111] R. Barbey, L. Lavanant, D. Paripovic, N. Schuwer, C. Sugnaux, S. Tugulu, H. A. Klok, Chem. Rev. 2009, 109, 5437-5527.

[112] C. M. Stafford, A. Y. Fadeev, T. P. Russell, T. J. McCarthy, Langmuir 2001, 17, 6547-6552. 
[113] Z. Huang, H. Ji, J. W. Mays, M. D. Dadmun, Macromolecules 2008, 41, 1009-1018.

[114] B. Radhakrishnan, R. Ranjan, W. J. Brittain, Soft Matter 2006, 2, 386-396.

[115] G. Boven, M. Oosterling, G. Challa, A. J. Schouten, Polymer 1990, 31, 2377-2383.

[116] G. Kickelbick, U. Schubert, Monatsh. Chem. 2001, 132, 13-30.

[117] M. s. Czaun, L. s. Hevesi, M. Takafuji, H. Ihara, Macromolecules 2009, 42, 4539-4546.

[118] L. Feng, J. Ye, X. Qiang, H. Zhang, f. Appl. Polym. Sci. 2011, 121, 454-461.

[119] J. Pyun, K. Matyjaszewski, Chem. Mater. 2001, 13, 3436-3448.

[120] G. Zhai, W. H. Yu, E. T. Kang, K. G. Neoh, C. C. Huang, D. J. Liaw, Ind. Eng. Chem. Res. 2004, 43, 1673-1680.

[121] W. H. Yu, E. T. Kang, K. G. Neoh, Ind. Eng. Chem. Res. 2004, 43, 51945202.

[122] S. Edmondson, V. L. Osborne, W. T. S. Huck, Chem. Soc. Rev. 2004, 33, $14-22$.

[123] M. M. Titirici, B. Sellergren, Chem. Mater. 2006, 18, 1773-1779.

[124] W.-C. Wang, K.-G. Neoh, E.-T. Kang, Macromol. Rapid Commun. 2006, 27, 1665-1669.

[125] C. Z. Li, B. C. Benicewicz, Macromolecules 2005, 38, 5929-5936.

[126] B. Hojjati, R. H. Sui, P. A. Charpentier, Polymer 2007, 48, 5850-5858.

[127] R. Ranjan, W. J. Brittain, Macromol. Rapid Commun. 2007, 28, 2084-2089.

[128] R. Ranjan, W. J. Brittain, Macromolecules 2007, 40, 6217-6223.

[129] M. D. Rowe, B. A. G. Hammer, S. G. Boyes, Macromolecules 2008, 41, 4147-4157. 
[130] D. H. Nguyen, M. R. Wood, Y. Zhao, S. Perrier, P. Vana, Macromolecules 2008, 41, 7071-7078.

[131] Y. Li, B. C. Benicewicz, Macromolecules 2008, 41, 7986-7992.

[132] S. Perrier, P. Takolpuckdee, C. A. Mars, Macromolecules 2005, 38, 67706774.

[133] D. H. Nguyen, P. Vana, Polym. Adv. Technol. 2006, 17, 625-633.

[134] Y. L. Zhao, S. Perrier, Macromolecules 2006, 39, 8603-8608.

[135] Y. Zhao, S. Perrier, Macromol. Symp. 2007, 248, 94-103.

[136] Y. Zhao, S. Perrier, Macromolecules 2007, 40, 9116-9124.

[137] D. Boschmann, M. Mänz, A.-C. Pöppler, N. Sörensen, P. Vana, f. Polym. Sci. Part A: Polym. Chem. 2008, 46, 7280-7286.

[138] C. Li, J. Han, C. Y. Ryu, B. C. Benicewicz, Macromolecules 2006, 39, 31753183.

[139] C. H. Liu, C. Y. Pan, Polymer 2007, 48, 3679-3685.

[140] Y. Yang, Z. Yang, Q. Zhao, X. Cheng, S. C. Tjong, R. K. Y. Li, X. Wang, X. Xie, F. Polym. Sci. Part A: Polym. Chem. 2009, 47, 467-484.

[141] Y. Gnanou, M. Fontanille, Organic and Physical Chemistry of Polymers, John Wiley \& Sons, Inc., Hoboken, New Jersey, 2008.

[142] W. Hellerich, G. Harsch, E. Baur, Werkstoff-Führer Kunststoffe, Carl Hanser Verlag, Munich, 2010.

[143] J. Hachenberg, Dissertation, Göttingen University, 2006.

[144] M. Schwabe, Dissertation, Göttingen University, 2010.

[145] P. R. Couchman, Macromolecules 2002, 11, 1156-1161.

[146] A. L. Agapov, A. P. Sokolov, Macromolecules 2009, 42, 2877-2878.

[147] D. W. v. Krevelen, K. t. Nijenhuis, Properties of Polymers, Elsevier, Oxford, 2009. 
[148] N. G. McGrum, B. E. Read, G. Williams, Anelastic and Dielectric Effects in Polymeric Solids, John Wiley \& Sons, London, 1967.

[149] M. L. Cerrada, J. L. de la Fuente, E. L. Madruga, M. Fernández-García, Polymer 2002, 43, 2803-2810.

[150] T. F. Schatzki, f. Polym. Sci. 1962, 57, 337-356.

[151] R. F. Boyer, Rubber Chem. Technol. 1963, 36, 1303-1421.

[152] R. Rotzoll, P. Vana, Macromol. Rapid Commun. 2009, 30, 1989-1994.

[153] S. Beuermann, D. A. Paquet, J. H. McMinn, R. A. Hutchinson, Macromolecules 1996, 29, 4206-4215.

[154] R. A. Hutchinson, S. Beuermann, D. A. Paquet, J. H. McMinn, C. Jackson, Macromolecules 1998, 31, 1542-1547.

[155] J. M. Asua, S. Beuermann, M. Buback, P. Castignolles, B. Charleux, R. G. Gilbert, R. A. Hutchinson, J. R. Leiza, A. N. Nikitin, J.-P. Vairon, A. M. v. Herk, Macromol. Chem. Phys. 2004, 205, 2151-2160.

[156] M. Buback, A. Feldermann, C. Barner-Kowollik, I. Lacik, Macromolecules 2001, 34, 5439-5448.

[157] D. Li, N. Li, R. A. Hutchinson, Macromolecules 2006, 39, 4366-4373.

[158] M. Buback, E. Müller, Macromol. Chem. Phys. 2007, 208, 581-593.

[159] M. Buback, F. D. Kuchta, Macromol. Chem. Phys. 1995, 196, 1887-1898.

[160] S. Beuermann, Macromol. Rapid Commun. 2009, 30, 1066-1088.

[161] I. Lacik, S. Beuermann, M. Buback, Macromolecules 2003, 36, 9355-9363.

[162] I. Lacik, S. Beuermann, M. Buback, Macromol. Chem. Phys. 2004, 205, 1080-1087.

[163] S. Beuermann, M. Buback, P. Hesse, I. Lacik, Macromolecules 2005, 39, 184-193.

[164] S. A. Seabrook, M. P. Tonge, R. G. Gilbert, F. Polym. Sci. Part A: Polym. Chem. 2005, 43, 1357-1368. 
[165] M. Stach, I. Lacik, D. Chorvat, M. Buback, P. Hesse, R. A. Hutchinson, L. Tang, Macromolecules 2008, 41, 5174-5185.

[166] S. Beuermann, M. Buback, C. Schmaltz, Macromolecules 1998, 31, 80698074.

[167] S. Beuermann, M. Buback, C. Schmaltz, F. D. Kuchta, Macromol. Chem. Phys. 1998, 199, 1209-1216.

[168] S. Beuermann, M. Buback, D. Nelke, Macromolecules 2001, 34, 6637-6640.

[169] S. Beuermann, M. Buback, C. Isemer, I. Lacik, A. Wahl, Macromolecules 2002, 35, 3866-3869.

[170] S. Harrisson, S. R. Mackenzie, D. M. Haddleton, Macromolecules 2003, 36, 5072-5075.

[171] I. Woecht, G. Schmidt-Naake, S. Beuermann, M. Buback, N. Garcia, f. Polym. Sci. Part A: Polym. Chem. 2008, 46, 1460-1469.

[172] M. Jung, I. van Casteren, M. J. Monteiro, A. M. van Herk, A. L. German, Macromolecules 2000, 33, 3620-3629.

[173] M. Jung, E. M. S. v. Hamersveld, T. Julien, A. M. v. Herk, Macromol. Rapid Commun. 2001, 22, 978-982.

[174] J. N. Coleman, U. Khan, Y. K. Gun'ko, Adv. Mater. 2006, 18, 689-706.

[175] U. Bergert, S. Beuermann, M. Buback, C. H. Kurz, G. T. Russell, C. Schmaltz, Macromol. Rapid Commun. 1995, 16, 425-434.

[176] J. D. Cho, H. T. Ju, J. W. Hong, f. Polym. Sci. Part A: Polym. Chem. 2005, 43, 658-670.

[177] R. Rotzoll, D. H. Nguyen, P. Vana, Macromol. Symp. 2009, 275-276, 1-12.

[178] A. M. v. Herk, Macromol. Rapid Commun. 2001, 22, 687-689.

[179] P. G. de Gennes, Macromolecules 1980, 13, 1069-1075.

[180] D. Dukes, Y. Li, S. Lewis, B. Benicewicz, L. Schadler, S. K. Kumar, Macromolecules 2010, 43, 1564-1570. 
[181] O. F. Olaj, P. Vana, M. Zoder, A. Kornherr, G. Zifferer, Macromol. Rapid Commun. 2000, 21, 913-920.

[182] O. F. Olaj, P. Vana, M. Zoder, Macromolecules 2002, 35, 1208-1214.

[183] O. F. Olaj, M. Zoder, P. Vana, A. Kornherr, I. Schnoll-Bitai, G. Zifferer, Macromolecules 2005, 38, 1944-1948.

[184] B. Abramoff, J. Covino, f. Appl. Polym. Sci. 1992, 46, 1785-1791.

[185] R. J. Zhou, T. Burkhart, fournal of Materials Science 2010, 45, 3016-3022.

[186] R. Y. Hong, H. P. Fu, Y. J. Zhang, L. Liu, J. Wang, H. Z. Li, Y. Zheng, f. Appl. Polym. Sci. 2007, 105, 2176-2184.

[187] S. H. Ahn, S. H. Kim, S. G. Lee, J. Appl. Polym. Sci. 2004, 94, 812-818.

[188] D. Shamir, A. Siegmann, M. Narkis, f. Appl. Polym. Sci. 2010, 115, 19221928.

[189] X. Y. Sun, Y. W. Luo, R. Wang, B. G. Li, B. Liu, S. P. Zhu, Macromolecules 2007, 40, 849-859.

[190] M. Schwabe, R. Rotzoll, S. Kuchemann, K. Nadimpalli, P. Vana, K. Samwer, Macromol. Chem. Phys. 2010, 211, 1673-1677.

[191] A. Omayu, S. y. Yoshioka, A. Matsumoto, Macromol. Chem. Phys. 2009, 210, 1210-1217.

[192] C. O. M'Bareck, Q. T. Nguyen, M. Metayer, J. M. Saiter, M. R. Garda, Polymer 2004, 45, 4181-4187.

[193] P. Eyerer, J. Woidasky, Einführung in Polymer Engineering, SpringerVerlag, Berlin Heidelberg, 2008.

[194] J. R. Schaefgen, I. M. Sarasohn, J. Polym. Sci. 1962, 58, 1049-1061.

[195] B. Metin, F. D. Blum, f. Chem. Phys. 2006, 124, 10.

[196] S. Krause, J. J. Gormley, N. Roman, J. A. Shetter, W. H. Watanabe, f. Polym. Sci. Part A: Gen. Pap. 1965, 3, 3573-3586. 
[197] R. J. Eldridge, F. E. Treloar, f. Polym. Sci. Polym. Chem. 1976, 14, 28312834.

[198] D. Kukulj, T. P. Davis, R. G. Gilbert, Macromolecules 1998, 31, 994-999.

[199] O. Custance, R. Perez, S. Morita, Nat. Nanotechnol. 2009, 4, 803-810.

[200] A. V. Rzhanov, K. K. Svitashev, L. Marton, C. Marton, "Ellipsometric Techniques to Study Surfaces and Thin Films", in Advances in Electronics and Electron Physics, Academic Press, 1979, p. 1-84.

[201] S. W. Hell, J. Wichmann, Opt. Lett. 1994, 19, 780-782.

[202] Q. P. Rong, A. P. Zhu, T. Zhong, f. Appl. Polym. Sci. 2011, 120, 3654-3661.

[203] B. Ebeling, M. Eggers, P. Vana, Macromolecules 2010, 43, 10283-10290.

[204] B. Ebeling, P. Vana, Polymers 2011, 3, 719-739.

[205] D. A. Smith, Makromol. Chem. 1967, 103, 301-303.

[206] S. Perrier, C. Barner-Kowollik, J. F. Quinn, P. Vana, T. P. Davis, Macromolecules 2002, 35, 8300-8306.

[207] D. Boschmann, Dissertation, Göttingen University, 2008.

[208] C. Strazielle, H. Benoit, O. Vogl, Eur. Polym. F. 1978, 14, 331-334.

[209] E. Penzel, N. Goetz, Angew. Makromol. Chem. 1990, 178, 191-200. 



\section{Appendices}

\section{A Kinetic Schemes for PREDICI ${ }^{\circledR}$}

\section{A.1 Basic Scheme}

Initiation on Surface

$\begin{array}{llll}\text { Initiator } & \rightarrow & 2 \mathrm{I}^{\bullet} & k_{\mathrm{d}} \cdot f \\ \mathrm{I}^{\bullet}+\mathrm{M} & \rightarrow & \mathrm{P}^{\bullet}(1) & \end{array}$

Pre-Equilibria

\begin{tabular}{|c|c|c|}
\hline $\mathrm{P}^{\bullet}(\mathrm{s})+\mathrm{RAFT}$ & $\rightarrow$ & $\operatorname{Int}^{\circ}(\mathrm{s})$ \\
\hline $\operatorname{Int}^{\bullet}(s)$ & $\rightarrow$ & $\mathrm{P}^{\bullet}(\mathrm{s})+\mathrm{RAFT}$ \\
\hline $\operatorname{Int}^{\bullet}(s)$ & $\rightarrow$ & polyRAFT(s) + $\mathrm{R}^{\bullet}$ \\
\hline $\operatorname{polyRAFT}(\mathrm{s})+\mathrm{R}^{\bullet}$ & $\rightarrow$ & $\operatorname{Int}^{\circ}(\mathrm{s})$ \\
\hline $\mathrm{PL}^{\bullet}(\mathrm{s})+\mathrm{RAFT}$ & $\rightarrow$ & $\operatorname{Int} \mathrm{L}^{\cdot}(\mathrm{s})$ \\
\hline $\operatorname{Int} \mathrm{L}^{\bullet}(\mathrm{s})$ & $\rightarrow$ & $\mathrm{PL}^{\bullet}(\mathrm{s})+\mathrm{RAFT}$ \\
\hline $\operatorname{IntL} \bullet(s)$ & $\rightarrow$ & polyRAFTL(s) + R $^{\bullet}$ \\
\hline polyRAFTL(s) + R $^{\bullet}$ & $\rightarrow$ & $\operatorname{IntL}{ }^{\circ}(\mathrm{s})$ \\
\hline $\mathrm{R}^{\bullet}+\mathrm{RAFT}$ & $\rightarrow$ & IntRR ${ }^{\bullet}$ \\
\hline IntRR・ & $\rightarrow$ & $\mathrm{R}^{\bullet}+\mathrm{RAFT}$ \\
\hline
\end{tabular}

Initiation through the Leaving Group in Solution

$\mathrm{R}^{\bullet}+\mathrm{M}$

$\rightarrow$

$\mathrm{PL}^{\bullet}(1)$

$k_{\mathrm{p}, \text { rein }}$

Propagation in Solution
$\mathrm{PL} \cdot(\mathrm{s})+\mathrm{M}$
$\rightarrow$
$\mathrm{PL} \cdot(\mathrm{s}+1)$
$k_{\mathrm{p}}$ 
Propagation on Surface
$\mathrm{P}^{\bullet}(\mathrm{s})+\mathrm{M}$
$\rightarrow \quad \mathrm{P}^{\bullet}(\mathrm{s}+1)$
$k_{\mathrm{p}}$

Main Equilibrium in Solution

$\begin{array}{llll}\mathrm{PL} \cdot(\mathrm{s})+\text { polyRAFTL }(\mathrm{r}) & \rightarrow & \operatorname{IntLLA}(\mathrm{s})+\operatorname{IntLLB}(\mathrm{r}) & k_{\mathrm{ad}} \\ \text { IntLLA(s) } & \rightarrow & \mathrm{PL} \cdot(\mathrm{s}) & \frac{1}{2} k_{\beta} \\ \text { IntLLB(s) } & \rightarrow & \mathrm{PL} \cdot(\mathrm{s}) & 1 / 2 k_{\beta} \\ \text { IntLLA(s) } & \rightarrow & \text { polyRAFTL(s) } & \frac{1}{2} k_{\beta} \\ \text { IntLLB(s) } & \rightarrow & \text { polyRAFTL(s) } & 1 / 2 k_{\beta}\end{array}$

Main Equilibrium between Solution and Surface

\begin{tabular}{|c|c|c|c|}
\hline polyRAFT(s) & $\rightarrow$ & IntOLA(s) & $k_{\mathrm{ad}, \mathrm{OL}} \cdot c_{\mathrm{PL}(\mathrm{s})}$ \\
\hline $\mathrm{PL}^{\bullet}(\mathrm{s})$ & $\rightarrow$ & IntOLB(s) & $k_{\mathrm{ad}, \mathrm{OL}} \cdot c_{\text {polyRAFT(s) }}$ \\
\hline $\mathrm{P}^{\bullet}(\mathrm{s})$ & $\rightarrow$ & IntOLC(s) & $k_{\mathrm{ad}, \mathrm{OL}} \cdot c_{\text {polyRAFTL(s) }}$ \\
\hline polyRAFTL(s) & $\rightarrow$ & IntOLD(s) & $k_{\mathrm{ad}, \mathrm{OL}} \cdot c_{\mathrm{P}(\mathrm{s})}$ \\
\hline IntOLA(s) & $\rightarrow$ & polyRAFT(s) & $1 / 2 k_{\beta}$ \\
\hline IntOLA(s) & $\rightarrow$ & $\mathrm{P}^{\bullet}(\mathrm{s})$ & $1 / 2 k_{\beta}^{p}$ \\
\hline IntOLB(s) & $\rightarrow$ & polyRAFTL(s) & $1 / 2 k_{\beta}^{\beta}$ \\
\hline IntOLB(s) & $\rightarrow$ & $\mathrm{PL}^{\bullet}(\mathrm{s})$ & $1 / 2 k_{\beta}^{p}$ \\
\hline IntOLC(s) & $\rightarrow$ & polyRAFT(s) & $1 / 2 k_{\beta}$ \\
\hline IntOLC(s) & $\rightarrow$ & $\mathrm{P}^{\bullet}(\mathrm{s})$ & $1 / 2 k_{\beta}^{p}$ \\
\hline IntOLD(s) & $\rightarrow$ & polyRAFTL(s) & $1 / 2 k_{\beta}$ \\
\hline IntOLD(s) & $\rightarrow$ & $\mathrm{PL}^{\bullet}(\mathrm{s})$ & $1 / 2 k_{\beta}$ \\
\hline
\end{tabular}

Main Equilibrium on Surface

$\begin{array}{llll}\mathrm{P}^{\bullet}(\mathrm{s})+\text { polyRAFT(r) } & \rightarrow & \operatorname{IntOOA}(\mathrm{s})+\operatorname{IntOOB}(\mathrm{r}) & k_{\mathrm{ad}, \mathrm{OO}} \\ \text { IntOOA(s) } & \rightarrow & \mathrm{P} \bullet(\mathrm{s}) & \frac{1}{2} k_{\beta} \\ \text { IntOOB(s) } & \rightarrow & \mathrm{P} \cdot(\mathrm{s}) & 1 / 2 k_{\beta} \\ \text { IntOOA(s) } & \rightarrow & \text { polyRAFT(s) } & \frac{1}{2} k_{\beta} \\ \text { IntOOB(s) } & \rightarrow & \text { polyRAFT(s) } & 1 / 2 k_{\beta}\end{array}$

\section{Termination}

$\begin{array}{llll}\mathrm{PL} \bullet(\mathrm{s})+\mathrm{PL} \cdot(\mathrm{r}) & \rightarrow & \text { PLLdead(s+r) } & k_{\mathrm{t}, \mathrm{LL}} \\ \mathrm{P}^{\bullet}(\mathrm{s})+\mathrm{P}^{\bullet}(\mathrm{r}) & \rightarrow & \operatorname{POOdead}(\mathrm{s}+\mathrm{r}) & k_{\mathrm{t}, \mathrm{OO}} \\ \mathrm{PL} \cdot(\mathrm{s})+\mathrm{P}^{\bullet}(\mathrm{r}) & \rightarrow & \text { POLdead(s+r) } & k_{\mathrm{t}, \mathrm{OL}} \\ \mathrm{R}^{\bullet}+\mathrm{R}^{\bullet} & \rightarrow & \text { RR } & k_{\mathrm{t}, \mathrm{LL}} \\ \mathrm{PL} \cdot(\mathrm{s})+\mathrm{R}^{\bullet} & \rightarrow & \text { PLLdead(s) } & k_{\mathrm{t}, \mathrm{LL}} \\ \mathrm{P}^{\bullet}(\mathrm{s})+\mathrm{R}^{\bullet} & \rightarrow & \text { POLdead(s) } & k_{\mathrm{t}, \mathrm{OL}}\end{array}$




\section{A.2 Extended Scheme for the IRT Model}

\section{Cross-Termination}

\begin{tabular}{|c|c|c|c|}
\hline $\mathrm{PL}^{\bullet}(\mathrm{s})+\operatorname{IntRR}{ }^{\bullet}$ & $\rightarrow$ & $\operatorname{Dead}(\mathrm{s})$ & $k_{\mathrm{t}, \text { cross }}$ \\
\hline $\operatorname{IntRR}^{\bullet}+\mathrm{R}^{\bullet}$ & $\rightarrow$ & Dead & $k_{\mathrm{t}, \text { cross }}$ \\
\hline $\mathrm{P}^{\bullet}(\mathrm{s})+\operatorname{IntRR}{ }^{\bullet}$ & $\rightarrow$ & $\operatorname{Dead}(\mathrm{s})$ & $k_{\mathrm{t}, \text { cross }}$ \\
\hline $\operatorname{Int}^{\circ}(\mathrm{s})+\mathrm{P}^{\bullet}(\mathrm{r})$ & $\rightarrow$ & $\operatorname{Dead}(\mathrm{s}+\mathrm{r})$ & $k_{\mathrm{t}, \text { cross }}$ \\
\hline $\operatorname{Int}^{\bullet}(\mathrm{s})+\mathrm{PL}^{\bullet}(\mathrm{r})$ & $\rightarrow$ & $\operatorname{Dead}(s+r)$ & $k_{\mathrm{t}, \text { cross }}$ \\
\hline $\operatorname{Int}^{\bullet}(s)+\mathrm{R}^{\bullet}$ & $\rightarrow$ & $\operatorname{Dead}(\mathrm{s})$ & $k_{\mathrm{t}, \text { cross }}$ \\
\hline $\operatorname{IntL}{ }^{\bullet}(\mathrm{s})+\mathrm{P}^{\bullet}(\mathrm{r})$ & $\rightarrow$ & $\operatorname{Dead}(s+r)$ & $k_{\mathrm{t}, \text { cross }}$ \\
\hline $\operatorname{IntL} L^{\bullet}(\mathrm{s})+\mathrm{PL} \cdot(\mathrm{r})$ & $\rightarrow$ & $\operatorname{Dead}(s+r)$ & $k_{\mathrm{t}, \text { cross }}$ \\
\hline $\operatorname{Int} L^{\bullet}(s)+\mathrm{R}^{\bullet}$ & $\rightarrow$ & $\operatorname{Dead}(\mathrm{s})$ & $k_{\mathrm{t}, \mathrm{cross}}$ \\
\hline $\operatorname{IntLLA}(s)+\mathrm{R}^{\bullet}$ & $\rightarrow$ & $\operatorname{Dead}(\mathrm{s})$ & $\frac{1}{2} k_{\mathrm{t}, \text { cross }}$ \\
\hline $\operatorname{IntLLA}(\mathrm{s})$ & $\rightarrow$ & $\operatorname{Dead}(\mathrm{s})$ & $1 / 2 k_{\mathrm{t}, \text { cross }} \cdot c_{\mathrm{R}}$ \\
\hline $\operatorname{IntLLA}(\mathrm{s})+\mathrm{PL}^{\bullet}(\mathrm{r})$ & $\rightarrow$ & $\operatorname{Dead}(s+r)$ & $1 / 2 k_{\mathrm{t}, \text { cross }}$ \\
\hline $\operatorname{IntLLA}(\mathrm{s})$ & $\rightarrow$ & $\operatorname{Dead}(\mathrm{s})$ & $\frac{1}{2} k_{\mathrm{t}, \text { cross }} \cdot c_{\mathrm{PL} \cdot \mathrm{r}}$ \\
\hline $\operatorname{IntLLA}(\mathrm{s})+\mathrm{P}^{\bullet}(\mathrm{r})$ & $\rightarrow$ & $\operatorname{Dead}(s+r)$ & $1 / 2 k_{\mathrm{t}, \text { cross }}$ \\
\hline IntLLA(s) & $\rightarrow$ & $\operatorname{Dead}(s)$ & $1 / 2 k_{\mathrm{t}, \text { rross }} \cdot c_{\mathrm{P}} \cdot$ \\
\hline $\operatorname{IntLLB}(\mathrm{s})+\mathrm{R}^{\bullet}$ & $\rightarrow$ & $\operatorname{Dead}(\mathrm{s})$ & $\frac{1}{2} k_{\mathrm{t}, \text { cross }}$ \\
\hline $\operatorname{IntLLB}(\mathrm{s})$ & $\rightarrow$ & $\operatorname{Dead}(\mathrm{s})$ & $1 / 2 k_{\mathrm{t} \text {,cross }} \cdot c_{\mathrm{R}}$ \\
\hline $\operatorname{IntLLB}(\mathrm{s})+\mathrm{PL}^{\bullet}(\mathrm{r})$ & $\rightarrow$ & $\operatorname{Dead}(s+r)$ & $1 / 2 k_{\mathrm{t}, \text { cross }}$ \\
\hline $\operatorname{IntLLB}(\mathrm{s})$ & $\rightarrow$ & $\operatorname{Dead}(\mathrm{s})$ & $1 / 2 k_{\mathrm{t}, \text { cross }} \cdot c_{\mathrm{PL}}$ \\
\hline $\operatorname{IntLLB}(\mathrm{s})+\mathrm{P}^{\bullet}(\mathrm{r})$ & $\rightarrow$ & $\operatorname{Dead}(s+r)$ & $1 / 2 k_{\mathrm{t}, \text { cross }}$ \\
\hline $\operatorname{IntLLB}(\mathrm{s})$ & $\rightarrow$ & $\operatorname{Dead}(s)$ & $1 / 2 k_{\mathrm{t}, \text { cross }} \cdot c_{\mathrm{P}} \cdot$ \\
\hline IntOLA(s) + $\mathrm{R}^{\bullet}$ & $\rightarrow$ & $\operatorname{Dead}(\mathrm{s})$ & $1 / 2 k_{\mathrm{t}, \text { cross }}$ \\
\hline IntOLA(s) & $\rightarrow$ & $\operatorname{Dead}(s)$ & $1 / 2 k_{\mathrm{t}, \text { cross }} \cdot c_{\mathrm{R}}$ \\
\hline $\operatorname{IntOLA}(\mathrm{s})+\mathrm{PL} \cdot(\mathrm{r})$ & $\rightarrow$ & $\operatorname{Dead}(s+r)$ & $1 / 2 k_{\mathrm{t}, \text { cross }}$ \\
\hline IntOLA(s) & $\rightarrow$ & $\operatorname{Dead}(s)$ & $1 / 2 k_{\mathrm{t}, \text { cross }} \cdot c_{\mathrm{PL}(\mathrm{r}}$ \\
\hline $\operatorname{IntOLA}(\mathrm{s})+\mathrm{P}^{\bullet}(\mathrm{r})$ & $\rightarrow$ & $\operatorname{Dead}(s+r)$ & $1 / 2 k_{\mathrm{t}, \text { cross }}$ \\
\hline IntOLA(s) & $\rightarrow$ & $\operatorname{Dead}(\mathrm{s})$ & $1 / 2 k_{\mathrm{t}, \text { cross }} \cdot c_{\mathrm{p}} \cdot$ \\
\hline $\operatorname{IntOLB}(\mathrm{s})+\mathrm{R}^{\bullet}$ & $\rightarrow$ & $\operatorname{Dead}(\mathrm{s})$ & $1 / 2 k_{\mathrm{t}, \text { cross }}$ \\
\hline IntOLB(s) & $\rightarrow$ & $\operatorname{Dead}(s)$ & $1 / 2 k_{\mathrm{t}, \text { cross }} \cdot c_{\mathrm{R}}$ \\
\hline $\operatorname{IntOLB}(\mathrm{s})+\mathrm{PL} \cdot(\mathrm{r})$ & $\rightarrow$ & $\operatorname{Dead}(s+r)$ & $1 / 2 k_{\mathrm{t}, \text { cross }}$ \\
\hline IntOLB(s) & $\rightarrow$ & $\operatorname{Dead}(\mathrm{s})$ & $1 / 2 k_{\mathrm{t}, \text { cross }} \cdot c_{\mathrm{PL}} \cdot$ \\
\hline $\operatorname{IntOLB}(\mathrm{s})+\mathrm{P}^{\bullet}(\mathrm{r})$ & $\rightarrow$ & $\operatorname{Dead}(s+r)$ & $1 / 2 k_{\mathrm{t}, \text { cross }}$ \\
\hline $\operatorname{IntOLB}(\mathrm{s})$ & $\rightarrow$ & $\operatorname{Dead}(\mathrm{s})$ & $1 / 2 k_{\mathrm{t}, \text { cross }} \cdot c_{\mathrm{P} \cdot(\mathrm{r})}$ \\
\hline IntOLC(s) + $\mathrm{R}^{\bullet}$ & $\rightarrow$ & $\operatorname{Dead}(s)$ & $\frac{1}{2} k_{\mathrm{t}, \text { cross }}$ \\
\hline IntOLC(s) & $\rightarrow$ & $\operatorname{Dead}(\mathrm{s})$ & $\frac{1}{2} k_{\mathrm{t}, \text { cross }} \cdot c_{\mathrm{R}}$ \\
\hline $\operatorname{IntOLC}(\mathrm{s})+\mathrm{PL}^{\bullet}(\mathrm{r})$ & $\rightarrow$ & $\operatorname{Dead}(s+r)$ & $1 / 2 k_{\mathrm{t}, \text { cross }}$ \\
\hline IntOLC(s) & $\rightarrow$ & $\operatorname{Dead}(s)$ & $1 / 2 k_{\mathrm{t}, \text { cross }} \cdot c_{\mathrm{PL}}$ \\
\hline $\operatorname{IntOLC}(\mathrm{s})+\mathrm{P}^{\bullet}(\mathrm{r})$ & $\rightarrow$ & $\operatorname{Dead}(s+r)$ & $1 / 2 k_{\mathrm{t}, \text { cross }}$ \\
\hline IntOLC(s) & $\rightarrow$ & $\operatorname{Dead}(\mathrm{s})$ & $1 / 2 k_{\mathrm{t}, \text { cross }} \cdot c_{\mathrm{P}^{*}(\mathrm{r})}$ \\
\hline
\end{tabular}




\begin{tabular}{|c|c|c|c|}
\hline $\operatorname{IntOLD}(\mathrm{s})+\mathrm{R}^{\bullet}$ & $\rightarrow$ & $\operatorname{Dead}(\mathrm{s})$ & $1 / 2 k_{\mathrm{t}, \text { cross }}$ \\
\hline IntOLD(s) & $\rightarrow$ & $\operatorname{Dead}(\mathrm{s})$ & $1 / 2 k_{\mathrm{t}, \text { cross }} \cdot c_{\mathrm{R}}$ \\
\hline $\operatorname{IntOLD}(\mathrm{s})+\mathrm{PL}^{\bullet}(\mathrm{r})$ & $\rightarrow$ & $\operatorname{Dead}(s+r)$ & $1 / 2 k_{\mathrm{t}, \text { cross }}$ \\
\hline IntOLD(s) & $\rightarrow$ & $\operatorname{Dead}(\mathrm{s})$ & $1 / 2 k_{\mathrm{t}, \text { cross }} \cdot c_{\mathrm{PL}}$ \\
\hline $\operatorname{IntOLD}(\mathrm{s})+\mathrm{P}^{\bullet}(\mathrm{r})$ & $\rightarrow$ & $\operatorname{Dead}(s+r)$ & $1 / 2 k_{\mathrm{t}, \text { cross }}$ \\
\hline IntOLD(s) & $\rightarrow$ & $\operatorname{Dead}(\mathrm{s})$ & $1 / 2 k_{\mathrm{t}, \text { cross }} \cdot c_{\mathrm{P}^{*}(\mathrm{r})}$ \\
\hline $\operatorname{IntOOA}(\mathrm{s})+\mathrm{R}^{\bullet}$ & $\rightarrow$ & $\operatorname{Dead}(\mathrm{s})$ & $1 / 2 k_{\mathrm{t}, \text { cross }}$ \\
\hline IntOOA(s) & $\rightarrow$ & $\operatorname{Dead}(\mathrm{s})$ & $1 / 2 k_{\mathrm{t}, \text { cross }} \cdot c_{\mathrm{R}}$ \\
\hline $\operatorname{IntOOA}(\mathrm{s})+\mathrm{PL}^{\bullet}(\mathrm{r})$ & $\rightarrow$ & $\operatorname{Dead}(s+r)$ & $1 / 2 k_{\mathrm{t}, \text { cross }}$ \\
\hline IntOOA(s) & $\rightarrow$ & $\operatorname{Dead}(\mathrm{s})$ & $1 / 2 k_{\mathrm{t}, \text { cross }} \cdot c_{\mathrm{PI}}$ \\
\hline $\operatorname{IntOOA}(\mathrm{s})+\mathrm{P}^{\bullet}(\mathrm{r})$ & $\rightarrow$ & $\operatorname{Dead}(s+r)$ & $1 / 2 k_{\mathrm{t}, \text { cross }}$ \\
\hline IntOOA(s) & $\rightarrow$ & $\operatorname{Dead}(\mathrm{s})$ & $1 / 2 k_{\mathrm{t}, \text { cross }} \cdot c_{\mathrm{P}}$. \\
\hline $\operatorname{IntOOB}(\mathrm{s})+\mathrm{R}^{\bullet}$ & $\rightarrow$ & $\operatorname{Dead}(\mathrm{s})$ & $1 / 2 k_{\mathrm{t}, \text { cross }}$ \\
\hline IntOOB(s) & $\rightarrow$ & $\operatorname{Dead}(s)$ & $1 / 2 k_{\mathrm{t}, \text { cross }} \cdot c_{\mathrm{R}}$ \\
\hline $\operatorname{IntOOB}(\mathrm{s})+\mathrm{PL}^{\bullet}(\mathrm{r})$ & $\rightarrow$ & $\operatorname{Dead}(s+r)$ & $1 / 2 k_{\mathrm{t}, \text { cross }}$ \\
\hline IntOOB(s) & $\rightarrow$ & $\operatorname{Dead}(\mathrm{s})$ & $1 / 2 k_{\mathrm{t}, \text { cross }} \cdot c_{\mathrm{PL}}$ \\
\hline $\operatorname{IntOOB}(\mathrm{s})+\mathrm{P}^{\bullet}(\mathrm{r})$ & $\rightarrow$ & $\operatorname{Dead}(s+r)$ & $1 / 2 k_{\mathrm{t}, \text { cross }}$ \\
\hline IntOOB(s) & $\rightarrow$ & $\operatorname{Dead}(\mathrm{s})$ & $1 / 2 k_{\mathrm{t}, \text { cross }} \cdot c_{\mathrm{P}} \cdot$ \\
\hline
\end{tabular}




\section{B Glossary}

$\begin{array}{ll}a & \text { Mark-Houwink parameter } \\ A_{0} & \text { cross section area } \\ \text { AA } & \text { acrylic acid } \\ \text { ACTA } & 4,4^{\prime} \text {-azobis(4-cyano- } N \text {-(3"-triethoxysilylpropyl)- } \\ & \text { valeric amide) } \\ \text { AFM } & \text { atomic force microscopy } \\ \text { AIBN } & 2,2^{\prime} \text {-azo-bis-(iso-butyronitrile) } \\ \text { ATRP } & \text { atom transfer radical polymerization } \\ \text { ca. } & \text { circa } \\ \text { cC } & \text { chemical confinement } \\ \text { CDB } & \text { cumyl dithiobenzoate } \\ \text { CSIRO } & \text { Commonwealth Science \& Industrial Research } \\ & \text { Organization } \\ c_{\mathrm{x}} & \text { concentration of species } \mathrm{x} \\ c_{\mathrm{x}}^{0} & \text { initial concentration of species x } \\ \delta & \text { phase angle, chemical shift } \\ \delta & \text { termination product formed by cross-termination } \\ \text { Dead } & \text { dimethyl-2,6-di(propyltrithiocarbonyl)heptanedioate } \\ \text { DPTH } & \text { sample standard deviation of the tensile modulus } \\ \Delta E & \text { sample standard deviation of the elongation at break } \\ \Delta \varepsilon_{\mathrm{B}} & \text { sample standard deviation of the elongation at yield } \\ \Delta \varepsilon_{\mathrm{Y}} & \text { deformation of a tensile specimen } \\ \Delta L & \text { sample standard deviation of the stress at break } \\ \Delta \sigma_{\mathrm{B}} & \text { sample standard deviation of the yield strength } \\ \Delta \sigma_{\mathrm{Y}} & \text { sample standard deviation of the toughness } \\ \Delta \tau & \text { dynamic mechanical analysis } \\ \mathrm{DMA} & \end{array}$


DMAc

DMPA

\section{E}

$E^{*}$

$\varepsilon$

$\dot{\varepsilon}$

$\varepsilon_{0}$

$\varepsilon_{\mathrm{B}}$

e.g.

EPR

$\varepsilon^{*}(t)$

et al.

$E^{\prime}$

$E^{\prime \prime}$

$\varepsilon_{\mathrm{Y}}$

f

F

$F_{\mathrm{AA}}$

$F_{\text {Sty }}$

$f_{\text {polystyrene }}^{\text {surface }}$

G

$\eta$

$[\eta]$

HPLC

HPT

$h_{\text {sample }}$

$h_{\text {sample }}^{0}$

$\mathrm{Hz}$

I

$i$

Int

IntL

IntLLA, IntLLB

IntOLA, IntOLB

IntOLC, IntOLD
$N, N$-dimethylacetamide

2,2-dimethoxy-2-phenylacetophenone

tensile modulus, Young's modulus

complex modulus

strain, elongation

rate of strain

maximum amplitude of strain

strain at break

exemplī grātia (for example)

electron paramagnetic resonance

strain in DMA

et alii, et alia (and others)

storage modulus

loss modulus

elongation at yield

initiator efficiency

force

molar AA content

molar styrene content

fraction of grafted polystyrene in composite after removal of free polymer

giga $\left(10^{9}\right)$

viscosity

intrinsic viscosity

high-performance liquid chromatography

hexyl(phenylethyl)trithiocarbonate

sample height

initial sample height

hertz $\left[\mathrm{s}^{-1}\right]$

initiator

chain length, square root of -1

intermediate on surface in RAFT pre-equilibrium

intermediate in solution in RAFT pre-equilibrium

fictive RAFT intermediate species for main equiblibrium in solution

fictive RAFT intermediate species for main equiblibrium

between surface and solution chains 
IntOOA, IntOOB fictive RAFT intermediate species for main equiblibrium on surface

IntRR RAFT intermediate formed by R-group attack

IRT intermediate radical termination

IUPAC International Union of Pure and Applied Chemistry

$j$

f

K

$k_{\text {ad }}$

$k_{\mathrm{ad}, 1}$

$k_{\text {ad,2 }}$

$k_{\text {ad,OL }}$

$k_{\text {ad,OO }}$

$k_{\text {ad,pre }}$

$k_{\beta}$

$k_{\beta, 1}, k_{\beta, 2}$

$k_{\mathrm{d}}$

$k_{\text {d,eff }}$

$K_{\text {eq }}$

$k_{\mathrm{i}}$

$k_{\mathrm{p}}$

$k_{\mathrm{p}, \mathrm{i}}$

$k_{\mathrm{p}, \text { rein }}$

$k_{\mathrm{t}}^{\mathrm{p},}$

$k_{\mathrm{t}, \mathrm{c}}$

$k_{\mathrm{t}, \mathrm{cros}}$

$k_{\mathrm{t}, \mathrm{d}}$

$k_{\mathrm{t}, \mathrm{LL}}$

$k_{\mathrm{t}, \mathrm{OL}}$

$k_{\mathrm{t}, \mathrm{OO}}$

$L_{0}$

$\log$

LVDT chains length

coupling constant

Mark-Houwink parameter

addition rate coefficient for RAFT main equilibrium rate coefficient for addition of macroradical to RAFT agent

rate coefficient for addition of $\mathrm{R}$-group radical to macromolecular RAFT agent

addition rate coefficient for RAFT main equilibrium between surface and solution

addition rate coefficient for RAFT main equilibrium on surface

addition rate coefficient for RAFT pre-equilibrium fragmentation rate coefficient of RAFT intermediate fragmentation rate coefficient of RAFT intermediate in pre-equilibrium

initiator decomposition rate coefficient

effective initiator decomposition rate coefficient

equilibrium constant for RAFT main equilibrium

initiation rate coefficient

propagation rate coefficient

propagation rate coefficient for initiation step

propagation rate coefficient for reinitiation step

termination rate coefficient

termination rate coefficient for combination

cross-termination rate coefficient

termination rate coefficient for disproportionation

termination rate coefficient in solution

termination rate coefficient between solution and surface

termination rate coefficient on surface

chain length at first inflection point in PLP-SEC, initial

length of specimen in tensile testing

logarithm to base 10

linear variable differential transformer 


\begin{tabular}{|c|c|}
\hline $\mathrm{m}$ & milli $\left(10^{-3}\right)$ \\
\hline$m$ & mass, chain length \\
\hline M & monomer, mega $\left(10^{6}\right)$ \\
\hline$M$ & molar mass \\
\hline$\mu$ & micro $\left(10^{-6}\right)$ \\
\hline MA & methyl acrylate \\
\hline MAA & methacrylic acid \\
\hline MCR & mid-chain radical \\
\hline MH & Mark-Houwink \\
\hline$M_{i}$ & molar mass of macromolecule with chain length $i$ \\
\hline$M_{\mathrm{M}}$ & molar mass of monomer \\
\hline MMD & molar-mass distribution \\
\hline$M_{n}$ & number-average molar mass \\
\hline$M_{\mathrm{n}}^{\text {solution }}$ & number-average molar mass in solution \\
\hline$M_{\mathrm{n}}^{\mathrm{n} u r f a c e}$ & number-average molar mass on surface \\
\hline$M_{\mathrm{n}}^{\text {theo }}$ & theoretical number-average molar mass \\
\hline$M_{\mathrm{RAFT}}$ & molar mass of RAFT agent \\
\hline$m^{\text {rel }}$ & relative mass \\
\hline$M_{\mathrm{w}}$ & weight-average molar mass \\
\hline $\mathrm{n}$ & nano $\left(10^{-9}\right)$ \\
\hline$n$ & normal, chain length \\
\hline$n_{\mathrm{abs}}$ & number of absorbed photons \\
\hline$n \mathrm{BA}$ & $n$-butyl acrylate \\
\hline$N_{i}$ & number of macromolecules with chain length $i$ \\
\hline NMP & nitroxide-mediated polymerization \\
\hline NMR & nuclear magnetic resonance \\
\hline $\mathrm{P}$ & polymer radical \\
\hline $\mathrm{Pa}$ & pascal \\
\hline PDI & polydispersity index \\
\hline PL & polymer radical in solution \\
\hline PLLdead & dead polymer in solution \\
\hline PLP & pulsed-laser polymerization \\
\hline$P_{\mathrm{n}}$ & number-average degree of polymerization \\
\hline POLdead & $\begin{array}{l}\text { dead polymer formed by termination between free and } \\
\text { grafted macroradicals }\end{array}$ \\
\hline $\operatorname{poly}(\mathrm{x})$ & polymer of monomer $\mathrm{x}$ \\
\hline polyRAFT & macromolecular RAFT agent on surface \\
\hline polyRAFTL & macromolecular RAFT agent in solution \\
\hline POOdead & dead polymer on surface \\
\hline ppm & parts per million \\
\hline
\end{tabular}




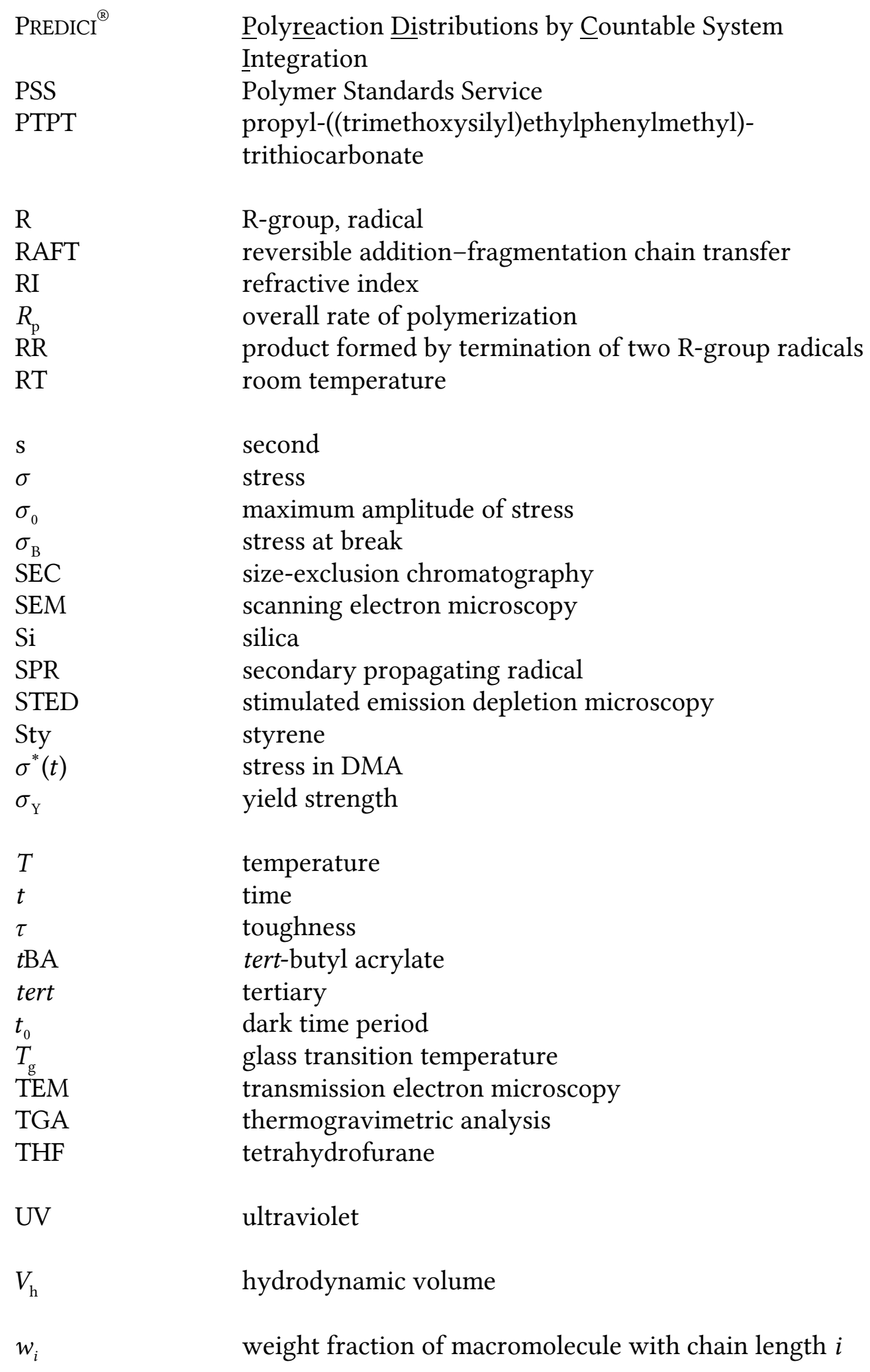




$\begin{array}{ll}w_{\mathrm{SiO}_{2}} & \text { weight fraction of silica } \\ \Phi & \text { primary quantum yield } \\ x_{i} & \text { amount fraction of macromolecule with chain length } i \\ X_{\mathrm{M}} & \text { total monomer conversion } \\ \mathrm{Z} & \text { Z-group } \\ \omega & \text { frequency }\end{array}$




\section{Danksagung}

Ich danke ganz besonders meinem Betreuer Prof. Dr. Philipp Vana für die interessante Themenstellung, sein großes Engagement während der letzten drei Jahre und die zahlreichen motivierenden Diskussionen.

Meinem Korreferenten und Zweitbetreuer Prof. Dr. Konrad Samwer möchte ich für das große Interesse an meiner Arbeit und für die exzellente Kooperation meinen tiefen Dank aussprechen.

Außerdem bedanke ich mich bei Dr. Moritz Schwabe für die fruchtbare Zusammenarbeit im Rahmen des Graduiertenkollegs GRK 782.

Stellvertretend für das Graduiertenkolleg GRK 782 möchte ich Prof. Dr. Martin Suhm für die finanzielle Unterstützung während der Promotion danken.

Herrn Dr. Hans-Peter Vögele danke ich für anregende Diskussionen, für technische und theoretische Hilfestellungen sowie für die zahlreichen Kalauer.

Für die schnelle und zuverlässige Arbeit durch die Glasbläserei möchte ich mich stellvertretend bei Herrn Hans-foachim Schlette bedanken.

Großer Dank gilt den Mitarbeiterinnen vom Analysenlabor des Instituts für Anorganische Chemie Frau Angelika Wraage, Frau Diana Kumpart und Frau Susanne Petrich für die stets zuverlässigen Elementaranalysen und TGAMessungen.

Der NMR-Abteilung des Instituts für Organische Chemie danke ich für die vielen gemessenen NMR-Spektren.

Bei Susanne Seyffarth möchte ich mich für die Aufnahme der sehr eindrucksvollen SEM-Bilder bedanken sowie für die produktive Zusammenarbeit im Rahmen des Graduiertenkollegs GRK 782.

Außerdem möchte ich Dr. Britta Fuchs für die stete Hilfsbereitschaft und die wunderbare Zusammenarbeit danken.

Ich danke Nadja Förster und Nils Wittenberg für die Hilfestellung bei der Synthese und Aufreinigung von ausgewählten Chemikalien.

Wibke Meiser danke ich für die umfangreiche Hilfe bei den PREDICI ${ }^{\circledR}-$ Simulationen sowie für das Korrekturlesen dieser Arbeit. 
Für das Korrekturlesen dieser Arbeit möchte ich außerdem Arne Wolpers, Bastian Ebeling und Jens Schrooten ganz herzlich danken.

Allen Mitgliedern der Arbeitsgruppen Makromolekulare Chemie und Technische und Makromolekulare Chemie danke ich für die entspannte und freundschaftliche Arbeitsatmosphäre. Speziell bedanke ich mich bei Björn Springer, Nicolai Sörensen, Aline Selke, Nils Wittenberg, Jan-Hendrik Schütz, Sebastian Primpke und Dr. Martin Mänz für die sehr zahlreichen lustigen Momente abseits der Arbeit. Bei meinen Bürokollegen Denis Yalalov und Felix Huff möchte ich mich für die entspannte und lustige Büroatmosphäre bedanken. Vielen Dank auch an Heike Rohmann und Sandra Lotze für praktische Hilfestellungen. Außerdem danke ich Cathrin Conrad und Björn Springer für die Bereitstellung eines komfortablen Büroplatzes während des Umbaus.

All meinen Freunden danke ich für die vielen schönen Zeiten in Hannover, Göttingen, Hamburg, Hof, München und wo es uns sonst noch hin verschlagen hat. Durch Euch konnte ich das Leben jenseits der Chemie voll und ganz genießen.

Sowohl meiner Familie als auch meiner Schwiegerfamilie danke ich für die stete Unterstützung während meines gesamten Studiums.

Abschließend danke ich ganz besonders meiner Frau Stephanie, die mich stets mit viel Geduld und Verständnis bei meiner Promotion unterstützt hat und immer aufzubauen wusste. Durch Dich ist das Leben in Göttingen erst so richtig schön geworden ist. Vielen Dank für alles! 


\title{
Lebenslauf
}

\author{
Persönliche Daten \\ geb. am 22.02.1983 in Hannover, Deutsch, verheiratet
}

Schule, Zivildienst

$06 / 2000$

11.06.2002

$07 / 2002-04 / 2003$

\section{Studium}

04/2003-04/2008

$04 / 2003-04 / 2005$

22.04.2005

$04 / 2005-04 / 2008$

$06 / 2007-12 / 2007$

23.04.2008

seit $05 / 2008$

05/2008-04/2011

seit $03 / 2010$

$10 / 2010-11 / 2010$

Göttingen, 21. Juni 2011
High School Diploma of Graduation, Sanger High School, Kalifornien

Abitur an der Albert-Einstein-Schule Laatzen, Hannover, Note: 1,1

Zivildienst im Stift zum Heiligen Geist, Hannover

Studium der Chemie an der Georg-August-Universität Göttingen

Grundstudium

Vordiplom, Note: „sehr gut“

Hauptstudium

Diplomarbeit: „Oberflächengebundene Polymerschlaufen durch kontrollierte radikalische RAFT-Polymerisation“ unter Anleitung von Prof. Dr. M. Buback, Note: „sehr gut“

Diplom-Chemiker, Note: „sehr gut“

Promotion am Institut für Physikalische Chemie unter der Anleitung von Prof. Dr. P. Vana

Stipendiat bzw. Kollegiat des Graduiertenkollegs 782 der Deutschen Forschungsgemeinschaft

Wissenschaftlicher Mitarbeiter am Institut für Physikalische Chemie der Georg-August-Universität Göttingen

Forschungsaufenthalt an der Tohoku University (Sendai) und Kyoto University, Japan. 